\title{
NOVEL DIP-PEN NANOLITHOGRAPHY STRATEGIES FOR NANOPATTERNING
}

Chien-Ching Wu 
Thesis committee members:

Prof. dr. K. J. Boller

Prof. dr. ir. D. N. Reinhoudt

Prof. dr. V. Subramaniam

Dr. A. H. Velders

Dr. C. Otto

Prof. dr. S. Speller

Prof. dr. H. Schönherr

Prof. dr. ir. H. J. W. Zandvliet

Prof. dr. J. J. L. M. Cornelissen
University of Twente (Chairman)

University of Twente (Promotor)

University of Twente (Promotor)

University of Twente (Assistent Promotor)

University of Twente (Assistent Promotor)

Radboud University Nijmegen

University of Siegen

University of Twente

University of Twente

This research has been financially supported by NanoNed, a national nanotechnology program coordinated by the Dutch Ministry of Economic Affairs (Project Number: 7124), and the MESA ${ }^{+}$Institute for Nanotechnology at the University of Twente.

\section{nano ned MESA+}

Publisher: Wöhrmann Print Service, Zutphen, The Netherlands

(c) Chien-Ching $\mathrm{Wu}$, Enschede, 2010

Cover Design: Chien-Ching Wu, Kai-Fan Chang

No part of this work may be reproduced by print, photocopy or any other means without the permission in writing of the author.

ISBN 978-90-365-3017-0 


\title{
NOVEL DIP-PEN NANOLITHOGRAPHY STRATEGIES FOR NANOPATTERNING
}

\author{
DISSERTATION
}

\author{
to obtain \\ the degree of doctor at the University of Twente, \\ on the authority of the rector magnificus, \\ Prof. dr. H. Brinksma, \\ on account of the decision of the graduation committee, \\ to be publicly defended \\ on Friday, June 18 ${ }^{\text {th }} 2010$, at 13:15
}

by

Chien-Ching $\mathrm{Wu}$

born on July $27^{\text {th }}, 1981$

in Kaohsiung City, Taiwan 
This dissertation has been approved by:

Prof. dr. ir. David N. Reinhoudt (Promotor)

Prof. dr. Vinod Subramaniam (Promotor)

Dr. Aldrik H. Velders (Assistent Promotor)

Dr. Cees Otto (Assistent Promotor) 
This thesis is dedicated to my family 給我摰愛的家人, 特別是阿公 



\section{Table of Contents}

$\begin{array}{lll}\text { Chapter } 1 & \text { General introduction } & 1\end{array}$

Chapter 2 Developments in Dip-Pen Nanolithography and Related 5

Chemical Strategies for Patterning Biomolecules

2.1 Introduction 6

2.2 Development of dip-pen nanolithography 6

2.3 Patterning biomolecules with dip-pen nanolithography 14

2.3.1 Indirect DPN Methods 17

2.3.1.1 Gold surfaces $\quad 17$

2.3.1.2 Silicon-based surfaces $\quad 21$

2.3.2 Direct DPN Methods 23

2.3.2.1 With bare tips 24

2.3.2.1a Gold surfaces $\quad 24$

2.3.2.1b Silicon-based surfaces $\quad 24$

2.3.2.1c Other surfaces 26

2.3.2.2 With functionalized tips 26

2.3.2.2a Gold surfaces 26

2.3.2.2b Silicon-based surfaces $\quad 27$

2.3.2.2c Other surfaces 29

2.4 Other AFM-based lithography techniques for patterning 30 biomolecules

2.5 Conclusions 33

$\begin{array}{lll}2.6 & \text { References } & 34\end{array}$

Chapter 3 Fabrication and Visualization of Metal-ion Patterns on Glass 43 by Dip-Pen Nanolithography

3.1 Introduction 44

3.2 Results and Discussion 46

3.2.1 The Atomic force fluorescence microscope (AFFM) 46

3.2.2 Sensitive monolayers 48

3.2.3 Fabrication and visualization of metal-ion patterns 51

3.2.3.1 Writing metal ions at micron scale 51 
3.2.3.2 Writing metal ions at sub-micron scale 52

3.3 Conclusions $\quad 54$

3.4 Experimental $\quad 54$

3.5 Acknowledgements 56

$\begin{array}{lll}3.6 & \text { References } & 56\end{array}$

Chapter 4 Protein Immobilization on Ni(II) Ion Patterns Prepared by 59

Microcontact Printing and Dip-Pen Nanolithography

4.1 Introduction 60

4.2 Results and Discussion $\quad 62$

4.2.1 Ni-NTA system 62

4.2.2 Fabrication of His-EGFP patterns by microcontact 63 printing $(\mu \mathrm{CP})$

4.2.3 Fabrication of His-EGFP patterns by dip-pen 64 nanolithography (DPN)

4.2.3.1 Writing with different contact times 65

4.2.3.2 Writing at various speeds 68

4.2.4 Fabrication of His-DsRed patterns by $\mu \mathrm{CP}$ and DPN 71

4.3 Conclusions $\quad 72$

$\begin{array}{lll}4.4 & \text { Experimental } & 72\end{array}$

$\begin{array}{lll}4.5 & \text { Acknowledgements } & 74\end{array}$

$\begin{array}{lll}4.6 & \text { References } & 74\end{array}$

Chapter 5 Reactive Microcontact Printing and Dip-Pen Nanolithography: 77 a strategy towards patterning submicrometer-sized orthogonal functional groups

$\begin{array}{lll}5.1 & \text { Introduction } & 78\end{array}$

5.2 Results and Discussion $\quad 80$

5.2.1 Fabrication of patterns with microcontact printing 81 $(\mu \mathrm{CP})$

5.2.1.1 Fabricating $\beta$-cyclodextrin ( $\beta C D$ ) patterns 81

5.2.1.2 Fabricating quantum dot patterns 83

5.2.1.3 Fabricating $\beta$-cyclodextrin and quantum 84 dot patterns together

5.2.2 Fabrication of patterns with dip-pen 86 nanolithography (DPN)

5.2.2.1 Fabricating quantum dot patterns 86

5.2.2.2 Fabricating $\beta$-cyclodextrin $(\beta C D)$ patterns $\quad 88$

5.3 Conclusions 
$\begin{array}{lll}5.4 & \text { Experimental } & 92\end{array}$

5.5 Acknowledgements 95

$\begin{array}{lll}5.6 & \text { References } & 95\end{array}$

Chapter 6 Porous Multilayer-coated AFM Tips for Dip-Pen 97 Nanolithography of Proteins

6.1 Introduction 98

6.2 Results and Discussion 99

6.2.1 Fabrication and characterization of porous 99 multilayer-coated AFM tip

6.2.2 Fabrication of protein patterns with 102 multilayer-coated AFM tip

6.2.2.1 His-EGFP patterns 103

6.2.2.2 His-DsRed patterns 104

6.2.3 Examining the co-deposition of the porous material 107

$\begin{array}{lll}6.3 \text { Conclusions } & 108\end{array}$

$\begin{array}{lll}6.4 & \text { Experimental } & 109\end{array}$

6.5 Acknowledgements 110

6.5 References 111

Chapter 7 Ink Loading of Porous Multilayer-coated AFM Tips 113

$\begin{array}{lll}7.1 & \text { Introduction } & 114\end{array}$

7.2 Results and Discussion 115

7.2.1 Fabrication of porous multilayer-coated AFM tips 115

7.2.2 Ink loading study of porous multilayer-coated AFM 120 tips

$\begin{array}{lll}7.3 & \text { Conclusions } & 123\end{array}$

$\begin{array}{lll}7.4 & \text { Experimental } & 124\end{array}$

7.5 References 125

Appendix 127

$\begin{array}{lr}\text { Summary } & 129\end{array}$

$\begin{array}{ll}\text { Samenvatting } & 133\end{array}$

$\begin{array}{ll}\text { Abbreviations } & 137\end{array}$

$\begin{array}{lr}\text { Acknowledgements } & 139\end{array}$ 
- iv - 


\section{Chapter 1}

\section{General Introduction}

Nanotechnology is a multidisciplinary research field aimed at manipulating matter at the nanometer scale, and even at an atomic or molecular level. ${ }^{1}$ One rapidly emerging area of nanotechnology in the past decade is nanofabrication, which aims at building structures with nanometer-scale features. The fabricated nanostructures can be integrated into complex hierarchical systems that provide a variety of applications, such as integrated circuits for the memory and processors of computers, optical components for display units, miniaturized sensors for environmental and chemical monitoring, and biochips for clinical diagnosis. $^{2}$ Therefore, nanofabrication begins to exert dramatic influences on our daily life.

Nanofabrication methods can be roughly divided into two categories: (1) top-down approaches, such as traditional photolithography, ${ }^{3}$ focused ion beam (FIB) lithography ${ }^{4}$ and nanoimprint lithography (NIL) ${ }^{5}$ that utilize irradiation or molding to reduce the lateral dimensions of a bulk substrate and to obtain a desired nanostructure, ${ }^{6}$ and (2) bottom-up approaches, such as microcontact printing $(\mu \mathrm{CP})^{7}$ and scanning tunneling microscopy (STM) lithography, ${ }^{8}$ that utilize self-assembly or manipulation of atoms or molecules to build up nanostructures. ${ }^{6}$

Dip-pen nanolithography (DPN) is an atomic force microscopy (AFM)-based lithography technique, which has the ability to fabricate patterns with a feature size down to $\sim 15 \mathrm{~nm}$ using both top-down and bottom-up approaches. ${ }^{9}, 10$ The serial nature of operation of DPN during its early development stages restricted the patterning efficiency. However, since the successful design and fabrication of AFM probe arrays, DPN has acquired the capacity to increase its throughput dramatically by generating patterns in parallel. ${ }^{11}$ Therefore, DPN has become a popular tool for nanofabrication. 
The research presented in this thesis focuses on two aspects: use of DPN to fabricate metal-ion and (bio)molecular patterns at micrometer or sub-micrometer scale, and development of AFM tip surface-modification methods for long-term DPN writing.

Chapter 2 is a literature review describing the development of the DPN technique in the past decade and the related chemical strategies that have been used to generate biomolecular architectures on solid surfaces with DPN. Some other AFM-based lithography techniques used for biopatterning are also briefly described at the end of the chapter.

Chapter 3 introduces a custom-built instrument, the atomic force fluorescence microscope (AFFM) implemented with a relative humidity ( $\mathrm{RH})$ control system, used for performing all the DPN experiments described in this thesis. AFFM allows one to write patterns and subsequently visualize in situ the written results in a sequential write-read mode. The controlled delivery of calcium metal ions with DPN on fluorescent sensitive monolayer-functionalized glass substrates is demonstrated by visualizing the modulation of the fluorescence intensity. The fabrication of metal ion patterns can be utilized to generate templates to immobilize biomolecules, and is described in detail in Chapter 4. Deposition of nickel ions onto nitrilotriacetic acid-terminated monolayer-functionalized glass substrates with DPN and $\mu \mathrm{CP}$ is demonstrated by immobilization of histidine-tagged visible fluorescent proteins via a specific metal-protein interaction. This approach also enabled us to characterize the dependence of transfer of nickel ions on fundamental parameters of DPN, such as writing speeds and tip-surface contact times.

Instead of using non-covalent interactions to generate patterns as presented in Chapter 3 and Chapter 4 , reactive $\mu \mathrm{CP}$ and $\mathrm{DPN}$ are demonstrated in Chapter 5. Amine containing $\beta$-cyclodextrin $\left(\beta C D-\mathrm{NH}_{2}\right)$ and quantum dots $\left(\mathrm{QD}-\mathrm{NH}_{2}\right)$ were microcontact printed and written onto $N, N$-carbonyldiimidazole-activated amino monolayerfunctionalized glass slides via the covalent formation of urethane bonds. Additionally, orthogonal microcontact printing of $\beta C D$ and QD- $\mathrm{NH}_{2}$ is also demonstrated.

In Chapter 6, a simple method is presented to coat the surfaces of AFM tips with a thin layer of porous film by using Layer-by-Layer ( $\mathrm{LbL})$ techniques. This porous film can easily absorb biomolecules and act as a larger volume ink reservoir compared to a bare AFM tip. Fluorescent protein patterns were generated at micron and sub-micron length scales by using the porous tips. A further investigation of the morphology variations of porous films-coated AFM tips, prepared under various conditions, is described in Chapter 7. These porous tips, inked with two kinds of visible fluorescent proteins, are used to 
study the ink reservoir properties of the porous films used to functionalize the DPN tips.

\section{References}

1. Bhushan, B., Springer Handbook of Nanotechnology. $2^{\text {nd }}$ ed.; Springer Science+Business Media, Inc.: 2007.

2. Geissler, M.; Xia, Y. N. Patterning: Principles and Some New Developments. Adv. Mater. 2004, 16, 1249-1269.

3. Xia, Y. N.; Rogers, J. A.; Paul, K. E.; Whitesides, G. M. Unconventional Methods for Fabricating and Patterning Nanostructures. Chem. Rev. 1999, 99, 1823-1848.

4. Tseng, A. A. Recent Developments in Micromilling Using Focused Ion Beam Technology. J. Micromech. Microeng. 2004, 14, R15-R34.

5. Chou, S. Y.; Krauss, P. R.; Renstrom, P. J. Imprint Lithography with 25-Nanometer Resolution. Science 1996, 272, 85-87.

6. Whitesides, G. M.; Love, J. C. The Art of Building Small - Researchers Are Discovering Cheap, Efficient Ways to Make Structures Only a Few Billionths of a Meter Across. Scientific American 2001, 285, 38-47.

7. Xia, Y. N.; Whitesides, G. M. Soft Lithography. Angew. Chem. Int. Ed. 1998, 37, 551-575.

8. Tseng, A. A.; Notargiacomo, A.; Chen, T. P. Nanofabrication by Scanning Probe Microscope Lithography: A Review. J. Vac. Sci. Technol., B 2005, 23, 877-894.

9. Piner, R. D.; Zhu, J.; Xu, F.; Hong, S. H.; Mirkin, C. A. "Dip-Pen" Nanolithography. Science 1999, 283, 661-663.

10. Ginger, D. S.; Zhang, H.; Mirkin, C. A. The Evolution of Dip-Pen Nanolithography. Angew. Chem., Int. Ed. 2004, 43, 30-45.

11. Salaita, K.; Wang, Y. H.; Mirkin, C. A. Applications of Dip-Pen Nanolithography. Nat. Nanotechnol. 2007, 2, 145-155. 


\title{
Chapter 2
}

Developments in Dip-Pen Nanolithography and Related Chemical Strategies for Patterning Biomolecules

\begin{abstract}
Dip-pen nanolithography (DPN) is a high-resolution atomic force microscopy (AFM)-based lithography technique. It utilizes the water meniscus formed between an AFM tip and a substrate to transfer ink molecules onto surfaces. This chapter reviews the development of this technique and its application to fabricate patterns of biomolecules at micrometer and nanometer scale.
\end{abstract}




\subsection{Introduction}

Controlled micro- and nano-patterning of biomolecules on solid surfaces while simultaneously maintaining the activity and structural integrity of the molecules has attracted a lot of attention in recent years because of its potential applications in biotechnology and medicine, including production and preparation of biochips and biosensors, drug discovery, and fundamental studies of biological processes. ${ }^{1,2}$ There are various techniques to fabricate architectures of small scale, such as traditional photolithography, ${ }^{3,} 4$ microcontact/nanocontact printing $(\mu \mathrm{CP} / \mathrm{nCP}){ }^{5-7}$ nanoimprint lithography (NIL), ${ }^{8,9}$ electron-beam lithography, ${ }^{10-12}$ and scanning probe-lithography (SPL). ${ }^{13,14}$ One scanning probe technique, atomic force microscopy (AFM), was invented in 1986 and uses Van der Waals interactions to study surface properties. ${ }^{15}$ AFM has become a very popular technique to elucidate the nanometer scale topography of different materials including insulators, semiconductors, conductors, and biological molecules. Besides direct imaging, AFM has also been explored to manipulate atoms and molecules on surfaces. AFM-based lithography utilizes an AFM probe to create patterns chemically, mechanically or electrically. AFM-based lithography techniques such as dip-pen nanolithography (DPN), nanografting, nanoshaving, conductive AFM lithography and enzymatic lithography attract enormous interest due to the technical potential and relatively low cost. Among these techniques, DPN is a popular method for fabricating biomolecular patterns.

A comprehensive review of recent efforts in all directions mentioned above is beyond the scope of this chapter. Therefore, the focus in this chapter is on two aspects: the development of DPN and the use of DPN for generating biomolecular architectures on different solid surfaces. Finally, some other AFM-based lithography techniques used for the fabrication of bio-patterns will be briefly summarized.

\subsection{Development of dip-pen nanolithography}

Before dip-pen nanolithography (DPN) was officially introduced by the Mirkin group in 1999 for transferring 1-octadecanethiol (ODT) from an AFM tip to a gold surface, ${ }^{14}$ Jaschke et al. had reported the deposition of aggregates of ODT onto high-energy surfaces of mica in 1995. ${ }^{16}$ Although some of the conclusions of Jaschke et al. conflicted with the results reported by others, these disordered ODT deposits might be considered as the first 
nanopatterns written with DPN. ${ }^{16,17}$ It is known that condensation of water results in a capillary force between a tip and a substrate when scanning force microscopy (SFM) is conducted in air. This has a significant influence on the contrast and resolution of AFM and lateral force microscopy (LFM) images. ${ }^{18}$ In 1997, Piner et al. demonstrated how water was transported to and from polymeric and inorganic substrates by LFM via a narrow gap capillary. ${ }^{19}$ They concluded that the phenomenon of water transport depended upon the relative humidity $(\mathrm{RH})$, surface properties and dynamics of the tip motion. Although the main purpose of this work was to characterize the effects of water on LFM measurements, this research inspired the authors to explore the DPN technique further.

The basic idea of DPN is very similar to writing ink with a conventional quill pen. Figure 2.1 illustrates the general process of DPN. ${ }^{20} \mathrm{~A}$ water meniscus is formed while an ink-coated AFM probe is brought into contact with a substrate in an ambient environment. The ink molecules are transferred to the surface via this water "bridge". Depending on the precise nature of the molecule-substrate interaction, which can be either (electro-)chemisorption or physisorption, the ink molecules may become immobilized. ${ }^{14,21 \text {, }}$ 22

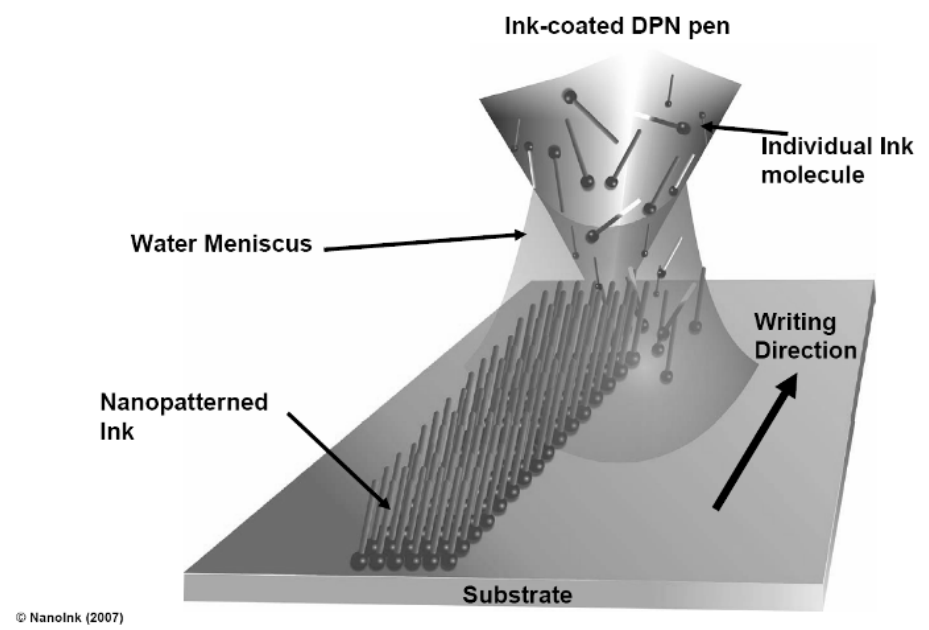

Figure 2.1. Schematic representation of molecular transport in the dip-pen nanolithography (DPN) process. Reprinted with permission from Ref. [20]. Copyright (2005) Elsevier. . 
The precise mechanism of ink transportation is complicated and different from one system to another. Over the past years, several groups have reported factors which have an influence on the deposition processes, including the chemical properties of the ink molecules and the chemical nature of the tip and the substrate, as well as the probe geometry, the ambient environment (temperature and relative humidity), and writing speed. $^{14,}$ 20-28 These conclusions are mostly based on studies of ODT or 16-mercaptohexadecanoic acid (MHA) deposited on gold surfaces. Haaheim et al. demonstrated that narrower MHA lines on gold surfaces were obtained by using sharper AFM tips or smoother surfaces. ${ }^{20}$ Many inks including organic molecules or bio-molecules have been used to demonstrate that longer tip-surface contact times or slower writing speeds results in larger feature sizes. ${ }^{14,23-25,29,30}$ However, this was not observed in all cases. A negligible dependence of the writing speed while depositing ODT on a Au surface at ambient RH was reported by Sheehan et al. ${ }^{26}$

Since DPN utilizes the water meniscus to deliver ink molecules, the shape and size of the water meniscus is another crucial issue. Weeks et al. used environmental scanning electron microscopy (ESEM) to image the formation of the water meniscus between an AFM tip and a substrate. ${ }^{31}$ The height of the meniscus is a function of RH as shown in Figure 2.2. There is no observation of meniscus formation while increasing RH until $70 \%$ with an $\mathrm{Au}$ substrate and $\sim 80 \%$ with a Si substrate. The height of the meniscus appears to increase exponentially with $\mathrm{RH}$ between $70 \%$ and $95 \%$ and is much larger than that calculated either using the Kelvin equation or Monte Carlo modeling. Because the resolution of ESEM is $\sim 50 \mathrm{~nm}$, these authors were not able to unequivocally visualize the formation of a meniscus at lower relative humidities. ${ }^{31}$ These results suggested that the transport rate of MHA does not change much at RH between $0 \%$ and $50 \%$, but abruptly increases while the RH is larger than $50 \% .{ }^{32}$ Later, the same authors used ESEM to study the dynamic growth of the water meniscus. They found that the water meniscus took many minutes to reach an equilibrium state when the tip was brought into contact with the surface. This observation suggests that the meniscus growth may account for the DPN ink transport. ${ }^{33}$ Rozhok et al. used ODT/MHA-Au systems to demonstrate how temperature and RH influence the growth rates of ODT and MHA. An increase of growth rate for both inks was observed with increasing temperature. However, only the growth rate of MHA rose with increasing $\mathrm{RH}$. These results suggest that the nature of the ink influences ink transport. In this case, the authors deduced that differences in behavior between ODT and MHA could be explained in terms of their different solubilities in the water meniscus. ${ }^{27}$ 


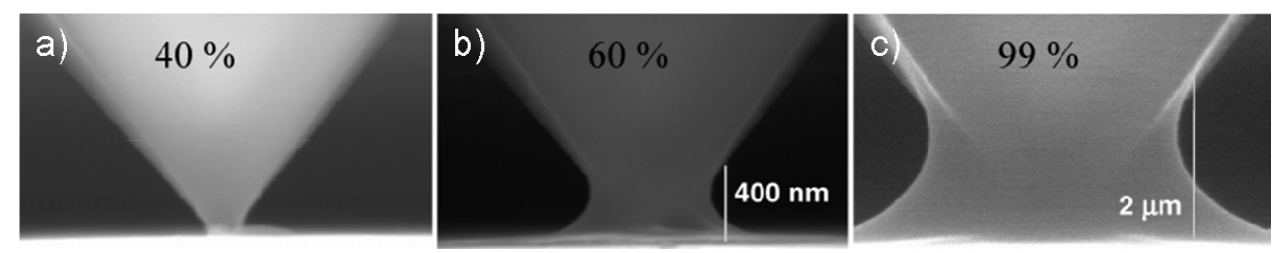

Figure 2.2. (a) - (c) ESEM images of water meniscus formed between an AFM tip and a substrate. Images collected at $5{ }^{\circ} \mathrm{C}$ at relative humidities of $40 \%, 60 \%$ and $99 \%$, respectively. Reprinted with permission from Ref. [31]. Copyright (2005), American Chemical Society.

According to the experimental DPN results, several models have been proposed to explain the ink transport. Jang et al. modeled the deposition of self-assembly molecules by DPN as a two-dimensional diffusion with a source. ${ }^{34}$ They treated the flow of ink from an AFM tip as a constant flux of molecules. Later, Sheehan et al. suggested that the process of deposition could be modeled as two-dimensional diffusion from an annular source of constant concentration with a fixed surface diffusion coefficient independent of $\mathrm{RH}^{26}$ In addition, Weeks et al. brought up a model that takes the kinetics of ink dissolution as well as the effect of RH and meniscus growth into account. ${ }^{31,33}$ However, none of these models could be used to explain all conditions. Therefore, a thorough understanding of the interplay of all the mechanisms will enable the development of more optimized ink-substrate combinations and achieve a higher resolution. ${ }^{21}$

DPN enables the preparation and registration of patterns from the sub $100-\mathrm{nm}$ to many micrometers length scale. The highest resolution achieved so far is with alkanethiol patterns fabricated on gold surfaces with a size of $\sim 15 \mathrm{~nm}$. Moreover, the flexibility of DPN allows the preparation of different patterns on substrates. Since its invention, DPN has been performed with a large variety of combinations of inks and substrates that are summarized in Table 2.1. 
Table 2.1. Overview of reported ink-substrate combinations which were used for DPN. ${ }^{35}$

\begin{tabular}{|c|c|}
\hline Ink molecules & Substrates \\
\hline Various Alkanethiols & $\mathrm{Au}$ \\
\hline Silane & $\mathrm{SiO}_{\mathrm{x}}$, Glass \\
\hline Silazanes & $\mathrm{SiO}_{\mathrm{x}}, \mathrm{GaAs}$ \\
\hline Alkynes & $\mathrm{Si}$ \\
\hline Cystamine & $\mathrm{Au}$ \\
\hline $\begin{array}{l}\text { Sol-Gels }\left(\mathrm{M}_{2} \mathrm{O}_{\mathrm{n}}, \mathrm{M}=\mathrm{Al}, \mathrm{Si}, \mathrm{Sn}\right) \text { or } \text { (Magnetic } \\
\text { nanostructures, } \mathrm{BaFe})\end{array}$ & $\mathrm{SiO}_{\mathrm{x}}$ \\
\hline $\begin{array}{l}\text { Nanoparticles (Q-Au); Gold Nanoclusters (thiol } \\
\text { capped) }\end{array}$ & $\mathrm{SiO}_{\mathrm{x}}$, Electrodes \\
\hline NanoParticles $\left(\mathrm{Fe}_{3} \mathrm{O}_{4}\right.$, magnetic, quantum dots) & $\mathrm{Si}, \mathrm{SiO}_{\mathrm{x}}, \mathrm{Mica}$ \\
\hline $\begin{array}{l}\text { Catalysts as sites for growing Polymer or } \mathrm{GaN} \\
\text { nanowires }\end{array}$ & $\mathrm{SiO}_{\mathrm{x}}$ \\
\hline $\begin{array}{l}\text { Gold }\left(\mathrm{HAuCl}_{4} \mathrm{Salt}_{\text {was }} \text { used }\right) ; \\
\text { Platinum }\left(\mathrm{H}_{2} \mathrm{PtCl}_{6} \text { salt was used }\right)\end{array}$ & $\mathrm{Si}$ \\
\hline Metal ions $\left(\mathrm{Ca}^{2+}, \mathrm{Ni}^{2+}\right)$ & silane modified $\mathrm{SiO}_{x}$ \\
\hline DNA & $\mathrm{Au}$, silane modified $\mathrm{SiO}_{\mathrm{x}}$ \\
\hline Proteins & $\mathrm{Au}, \mathrm{SiO}_{2}, \mathrm{NiO}_{\mathrm{x}}$, silane modified $\mathrm{SiO}_{\mathrm{x}}$ \\
\hline Collagen & $\mathrm{Au}$ \\
\hline Peptide & $\mathrm{Au}, \mathrm{Ni}, \mathrm{SiO}_{\mathrm{x}}$ \\
\hline Biotin & $\mathrm{SiO}_{\mathrm{x}}$, silanized modified glass \\
\hline poly(allylamine hydrochloride) (PAH) & $\mathrm{SiO}_{\mathrm{x}}$ \\
\hline poly (styrenesulfonate) (PSS) & $\mathrm{SiO}_{\mathrm{x}}$ \\
\hline Mercaptoimidazole & Gold \\
\hline Conducting Polymers (PolyPyrrole \& THF) & Gold electrodes \\
\hline Solid inks (Octadecylphosphonic Acid) & Mica \\
\hline Luminescent molecules (e.g. Rhodamine) & Glass \\
\hline Polymer (Nanowires, poly(thiophene)) & $\mathrm{SiO}_{\mathrm{x}}$ \\
\hline Dendrimers & $\mathrm{SiO}_{\mathrm{x}}$ \\
\hline Polylysine & Mica \\
\hline phospholipid & $\begin{array}{l}\mathrm{Si} \text {, glass, evaporated metal films, } \\
\text { polystyrene }\end{array}$ \\
\hline
\end{tabular}


The idea of DPN has been extended to several DPN-based techniques including nanopen reader and writer (NPRW), ${ }^{36,37}$ electrochemical DPN (E-DPN), ${ }^{38}$ electro pen nanolithography $(\mathrm{EPN})^{39}$ and scanning probe contact printing (SP-CP). ${ }^{40}$ The process of NPRW shown in Figure 2.3a is based on the combination of DPN and nanografting (Section 2.4). ${ }^{36}$ A tip was inked with molecules different from the molecules of a self-assembled monolayer (SAM) on the substrate. Subsequently, the tip was brought into contact with the surface at higher load. The pre-functionalized SAM was removed mechanically, followed immediately by the deposition of molecules from the tip. The E-DPN and EPN approaches have in common that a bias voltage between a (semi)conductive tip and a substrate is applied while patterning nanostructures. E-DPN utilizes the water meniscus as a tiny electrochemical cell where metal salts pre-coated on the tip, e.g. $\mathrm{H}_{2} \mathrm{PtCl}_{6}$, are dissolved and then reduced to metal that is deposited on the substrate. ${ }^{38}$ For EPN (Figure 2.3b), a voltage-biased tip is moved over a methyl-terminated SAM-functionalized surface. By electro-oxidation, the methyl groups are converted to reactive carboxylic acid groups which can be used to attach ink molecules in situ. ${ }^{39}$ SP-CP utilizes sharp or flat-topped DPN tips made of poly(dimethylsiloxane) (PDMS) to transfer ink onto a substrate. ${ }^{40}$

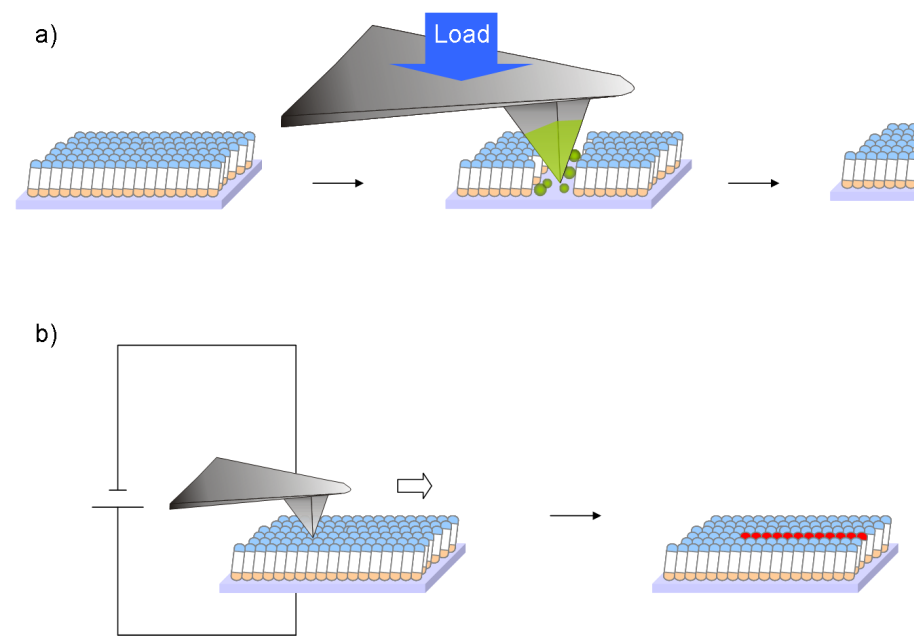

Figure 2.3. The schematic processes of (a) nanopen reader and writer (NPRW) (b) electro pen nanolithography (EPN). 
Although DPN can be regarded as an effective tool for nanofabrication, initially a major drawback, like all other scanning probe lithography systems, was that it is inherently a serial process, which limits the throughput. In order to increase the efficiency of DPN, two different systems have been developed: one with passive "duplicating" tip arrays and the other with individually actuated active tips. ${ }^{22}$

The first and simplest passive probe array implemented for parallel DPN was described by Hong et al. in $2000 .^{41}$ The pens were brought simultaneously into contact with a slightly tilted substrate and scanned together. This produced the same number of patterns as the number of tips in the array at the same time. The results showed that the feature size of patterns had less than $10 \%$ standard deviation in line-width, implying that line-widths in DPN experiments are not strongly dependent on the tip-substrate contact force at least within certain ranges. ${ }^{42}$ In designing passive probe arrays, the force constant has to be such that scratching of the substrate, oversensitivity to external noise, or difficulties in lifting the array due to large adhesive forces are prevented. ${ }^{43,44}$ Recently, passive probe arrays have been developed to write multiple arrays at one time and the number of probes of each array has dramatically increased to $\sim 55,000$ which allows users to obtain as many as $450,000,000$ sophisticated features at sub-100nm scale within 30 minutes. $^{22,45-47}$ Such an array has been used to pattern small molecules e.g. ODT and phospholipid membranes. ${ }^{45,48}$

In order to write different patterns simultaneously, the development of active arrays in which each tip can be controlled separately is necessary. Zou et al. have developed a conductive-based contact sensing system for probe arrays in DPN. ${ }^{49}$ This method uses the electrical resistance between the tip and the substrate as a sensitive indicator of physical contact and has the capability of switching each individual probe on/off independently based on electrical conduction. ${ }^{49}$ Another way for controlling each tip is using thermal bimorph actuation (Figure 2.4a). Heating an embedded electrical heater causes differential expansion of components and therefore leads to bending of the cantilever close to/away from the substrate. ${ }^{50-52}$ Since the distance between tips is fixed, it is possible to arrange the actions of tips spatially. Wang et al. used such a thermal bimorph actuator to develop an array consisting of different functional probes for nano-patterning and imaging shown in Figure 2.4b. ${ }^{53}$ Advantages of this device are that the cross contamination is minimized, improving the efficiency of operation and increasing the registration accuracy. Recently, the electrostatic mechanism has also been utilized to actuate DPN probe arrays. This mechanism does not require probe heating, which is necessary for the thermal bimorph 
actuation. In this way, the problem of cross talk between neighboring probes is reduced, which allows more closely spaced probe arrays. ${ }^{54}$

a)

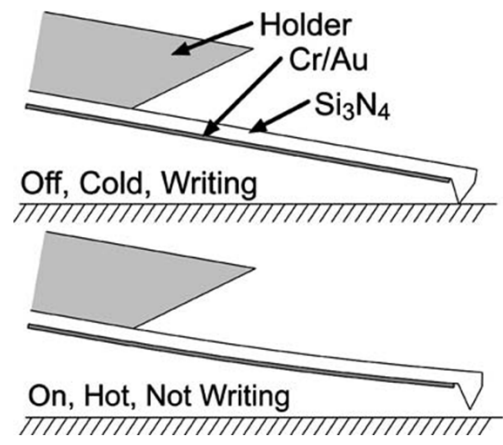

b)

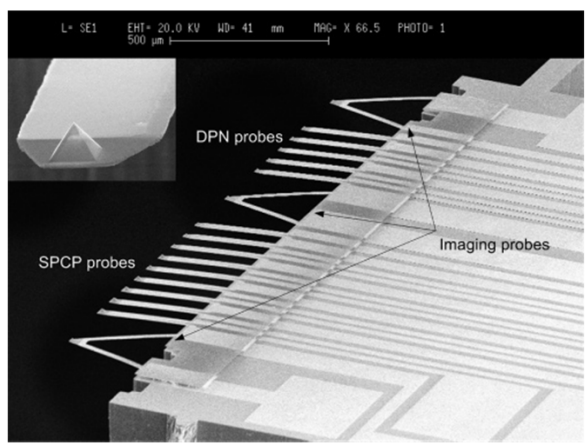

Figure 2.4. (a) The concept of operating an active DPN probe actuated by a thermal bimorph mechanism. Reprinted with permission from Ref. [50]. Copyright (2004) American Institute of Physics. (b) SEM image of a multifunctional probe array including five DPN Si $i_{3} N_{4}$ probes, nine SP-CP PDMS probes and three imaging $\mathrm{Si}_{3} \mathrm{~N}_{4}$ probes. The inset shows a $\mathrm{Si}_{3} \mathrm{~N}_{4}$ tip with a curvature radius of $100 \mathrm{~nm}$. Reprinted with permission from Ref. [53]. Copyright (2005) American Chemical Society.

All of these developments in DPN enable many applications in chemistry, materials sciences, and bio-sciences. ${ }^{21,22}$ Areas that are considered as unique applications of DPN include (1) fabricating biomolecular patterns as micro- and nano-arrays, (2) building chemical nanostructures as templates for the controlled orthogonal assembly of materials, and (3) generating hard features on surfaces such as etch-resist to obtain nanostructures with comparable feature sizes to those fabricated by e-beam lithography. ${ }^{21,22}$ In this chapter, the focus is on the first application, which will be described in detail in the next section. 


\subsection{Patterning biomolecules with dip-pen nanolithography}

The range of biomolecules is very broad including cellulose, lipids, peptides, DNA, saccharides, and proteins. To focus the scope of this review, only proteins ${ }^{23,24,30,55-79}$, peptides $^{80-89}$, and DNA ${ }^{29,90-92}$ used for patterning by DPN are discussed.

Generally, two strategies can be discerned to pattern biomolecules with the DPN technique, namely an indirect method and a direct method (Figure 2.5). The indirect method (Section 2.3.1) places a suitable molecular functionality at a surface. For instance, first a layer of protein-adherent molecules is fabricated, followed by the immobilization of proteins from solution. The direct method (Section 2.3.2) comprises direct deposition of the biomolecule from an ink-coated tip onto a surface. ${ }^{1,2}$

Functional immobilization of biomolecules, especially proteins, onto solid surfaces is very challenging since the interaction of proteins with a bare solid substrate frequently results in denaturation of proteins. ${ }^{24} \mathrm{~A}$ variety of chemical strategies, sorted by non-covalent and covalent approaches, have been utilized in either indirect or direct DPN for patterning biomolecules, and are summarized in Table 2.2 and 2.3, respectively. In general, two main types of substrates, gold and silicon-based substrates have been used. Therefore, in each subsection, the chemical strategies utilized for patterning biomolecules are discussed based on the classification of the substrates used. In addition to the approaches summarized here, there are many other methods employed to fabricate biomolecular patterns onto a solid support using other lithography techniques, which have been described in several review articles and may also be applied to DPN in the near future. $^{93-96}$

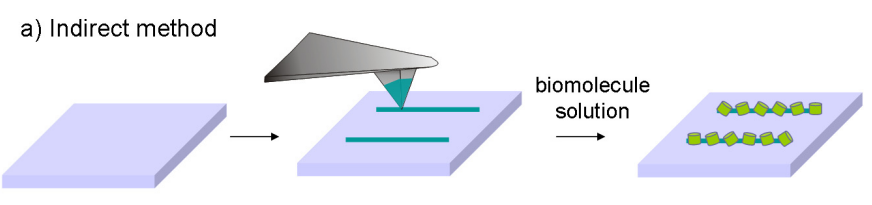

b) Direct method

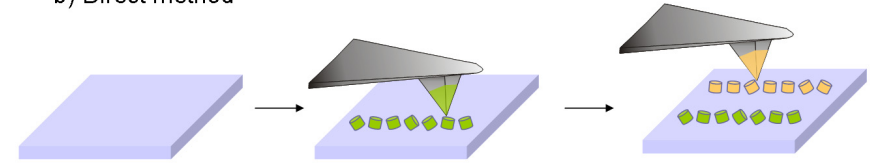

Figure 2.5. Schematic representation of (a) indirect and (b) direct dip-pen nanolithography (DPN) method for patterning biomolecules. 
Table 2.2. Overview of reported chemical strategies utilized for indirect DPN for patterning biomolecules on solid surface.

\begin{tabular}{|c|c|c|c|}
\hline \multicolumn{4}{|c|}{ Indirect DPN: non-covalent attachments } \\
\hline Mechanism & \multicolumn{2}{|r|}{ Examples } & Surfaces ${ }^{*}$ \\
\hline \multirow[t]{2}{*}{$\begin{array}{l}\text { Electrostatic or } \\
\text { hydrophobic interaction }\end{array}$} & $>$ & $\begin{array}{l}\text { Immunoglobulin G }(\operatorname{IgG})^{60,} \quad 66, \quad{ }^{69}, \text { lysozyme }^{60} \text {, } \\
\text { retronectin } \\
\text { (CPMV) nanoparticles }^{55} \text { on carboxylic acid-terminated } \\
\text { surface. } \\
\text { Membrane protein complexes }{ }^{65} \text { on protonated } \\
\text { amino-terminated surface. }\end{array}$ & $\mathrm{Au}$ \\
\hline & & $\begin{array}{l}\text { Negatively charged } \mathrm{DNA}^{90,92} \text { on poly(allylamine } \\
\text { hydrochloride) (PAH) }\end{array}$ & Si-based \\
\hline \multirow{2}{*}{$\begin{array}{l}\text { Biotin-streptavidin } \\
\quad \text { interaction }\end{array}$} & $>$ & $\begin{array}{l}\text { Biotinylated bovine serum albumin (Biotin-BSA })^{67} \text { on } \\
\text { streptavidin-terminated surface. } \\
\text { Streptavidin }{ }^{67,75,79} \text { biotin-terminated surface. }\end{array}$ & $\mathrm{Au}$ \\
\hline & $>$ & 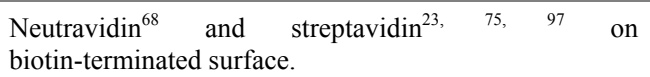 & Si-based \\
\hline \multirow[t]{2}{*}{ Coordination chemistry } & $>$ & $\begin{array}{l}\text { Tobacco mosaic virus (TMV) }{ }^{70} \text { on } \mathrm{Zn}(\mathrm{II}) \text {-carboxylic } \\
\text { acid rich-terminated surface. } \\
\text { Histidine (His)-tagged ifnar } 2^{79} \text { on } \mathrm{Ni}^{\mathrm{BT}} \text { trisnickel(II)- } \\
\text { nitrilotriacetic acid (NTA)-terminated surface. }\end{array}$ & $\mathrm{Au}$ \\
\hline & $>$ & His-VFPs ${ }^{63,74,75,78}$ on Ni-NTA- terminated surface. & Si-based \\
\hline ELP fusion & & $\begin{array}{l}\text { ELP fusion proteins }{ }^{87} \text { on elastin-like polypeptides } \\
\text { (ELP). }\end{array}$ & $\mathrm{Au}$ \\
\hline Others (not specified) & $>$ & $\begin{array}{l}\text { Antibodies }{ }^{72} \text {, e.g. } \operatorname{IgY}, \operatorname{IgM} \text { on } \mathrm{Zn}(\mathrm{II}) \text {-carboxylic acid } \\
\text { rich-terminated surface. }\end{array}$ & $\mathrm{Au}$ \\
\hline \multicolumn{4}{|c|}{ Indirect DPN: covalent attachments } \\
\hline \multirow{2}{*}{ Thioether bond formation } & & $\begin{array}{l}\text { Cysteine mutant Cowpea mosaic virus (Cys-CPMV) } \\
\text { nanoparticles }{ }^{55,76} \text { on maleimide-terminated surface. }\end{array}$ & $\mathrm{Au}$ \\
\hline & $>$ & Biotin-maleimide ${ }^{23,97}$ on thiol-terminated monolayer. & Si-based \\
\hline \multirow[t]{2}{*}{ Amide bond formation } & & $\begin{array}{l}\text { Amine-containing } \mathrm{DNA}^{91} \text { on carboxylic acid terminated } \\
\text { monolayer } \\
\text { protein } \mathrm{A} / \mathrm{G}^{71} \text { and amine-containing biotin } \\
\text { (ELPs }^{67} \text {, peptide } \\
\text { ester-terminated monolayer. }\end{array}$ & $\mathrm{Au}$ \\
\hline & & $\begin{array}{l}\text { NHS functionalized biotin on polyethylenimine } \\
\text { (PEI)-sputtered surface. }{ }^{68}\end{array}$ & Si-based \\
\hline Oxyme formation & $>$ & $\begin{array}{l}\text { Oxyamine-terminated linear } \quad \text { peptide }^{83} \\
\text { quinone-terminated surface. }\end{array}$ & $\mathrm{Au}$ \\
\hline $\begin{array}{l}\text { Ring opening } \\
\text { polymerization (ROP) }\end{array}$ & & $\begin{array}{l}\text { tryptophan- } N \text {-carboxyanhydride (Trp-NCA) on } \mathrm{G} 4-\mathrm{NH}_{2} \\
\text { polyamidoamine (PAMAM) dendrimers deposited } \\
\text { surface. }^{81}\end{array}$ & Si-based \\
\hline
\end{tabular}

\footnotetext{
* Si-based surfaces include silicon, silicon oxide and glass.
} 
Table 2.3. Overview of reported chemical strategies utilized for direct DPN for patterning biomolecules on solid surface.

\begin{tabular}{|c|c|c|c|}
\hline \multicolumn{4}{|c|}{ Direct DPN: non-covalent attachments } \\
\hline Mechanism & & Examples & Surfaces \\
\hline $\begin{array}{l}\text { Electrostatic or hydrophobic } \\
\text { interaction }\end{array}$ & & $\begin{array}{l}\operatorname{IgG}^{59} \text { on negatively charged } \mathrm{SiO}_{2} \text { surface } \\
\mathrm{IgG}^{64} \text { and His-VFPs }{ }^{63} \text { on amino-terminated surface. }\end{array}$ & Si-based \\
\hline $\begin{array}{l}\text { Crown ether to protonated } \\
\text { amine interaction }\end{array}$ & $>$ & $\begin{array}{l}\text { Angiogenin }{ }^{56} \text { and integrin } \alpha_{\mathrm{v}} \beta_{3}{ }^{56} \text { on Prolinker }{ }^{\mathrm{TM}} \text {-coated } \\
\text { surface. }\end{array}$ & $\mathrm{Au}$ \\
\hline coordination chemistry & & $\begin{array}{l}\text { His-VFPs }{ }^{63,74,75} \text { on Ni-NTA-terminated surface. } \\
\text { His-ubiquitin }{ }^{58} \text { and thioredoxin }{ }^{58} \text { on nickel oxide } \\
\text { surface. }\end{array}$ & Si-based \\
\hline \multirow{4}{*}{ Others (not specified) } & $>$ & 3RGBP1 peptides ${ }^{80}$ on Au surface. & $\mathrm{Au}$ \\
\hline & & $\begin{array}{l}\text { QBP1 }{ }^{80}, \mathrm{QBP}^{80} \text { peptides and amphiphile (PA) } \\
\text { nanofibers }{ }^{86} \text { on Si surface. }\end{array}$ & Si-based \\
\hline & & Antibody ${ }^{77}$ and streptavidin ${ }^{77}$ on DNA. & \multirow[b]{2}{*}{ biosurface } \\
\hline & $>$ & $\begin{array}{l}\text { Biotin-collagen-binding peptides onto inner collagenous } \\
\text { layer of Brunch's membrane (ICLBM) }\end{array}$ & \\
\hline \multicolumn{4}{|c|}{ Direct DPN: covalent attachments } \\
\hline Thioether bond formation & $>$ & $\begin{array}{l}\text { TAT peptide with cysteine } \text { residues }^{85} \text { on } \\
\text { maleimide-terminated surface. }\end{array}$ & Si-based \\
\hline Au-thiol interaction & $>$ & $\begin{array}{l}\text { Thiol-containing collagen }{ }^{62} ; \mathrm{DNA}^{29}, \mathrm{IgG}^{30}, \text { lysozyme }^{30} \\
\text { TAT peptide } \\
\text { surface. }\end{array}$ & $\mathrm{Au}$ \\
\hline Michael addition & $>$ & $\begin{array}{l}\text { Acrylamide-containing } \mathrm{DNA}^{29} \text { and oligonucleotide }{ }^{73} \text { on } \\
\text { thiol-terminated surface. }\end{array}$ & Si-based \\
\hline Amide bond formation & & $\begin{array}{l}\text { Amine-containing } \mathrm{DNA}^{24} \text { and } \mathrm{IgG}^{24} \text { on NHS ester- } \\
\text { terminated surface. }\end{array}$ & Si-based \\
\hline Imine bond formation & $>$ & $\begin{array}{l}\text { Amine-containing } \operatorname{IgG}^{59} \text { on aldehyde-terminated } \\
\text { monolayer }\end{array}$ & Si-based \\
\hline Epoxy to amine reaction & $>$ & $\begin{array}{l}\text { Amine-containing } \mathrm{HCG}^{61} \text { onto epoxy-terminated } \\
\text { surface. }\end{array}$ & Si-based \\
\hline
\end{tabular}

* Si-based surfaces include silicon, silicon oxide and glass. 


\subsubsection{Indirect DPN Methods}

\subsubsection{Gold surfaces}

The indirect method offers a very elegant and site-specific approach for biomolecule patterning. Several indirect DPN methods utilize 16-mercaptohexadecanoic acid (MHA) as ink molecules to generate dot or line patterns on gold surfaces. Subsequently, the non-patterned areas are passivated with ODT to prevent the non-specific adsorption of biomolecules. The carboxylic acid-terminated groups of MHA are subsequently used to immobilize biomolecules via electrostatic or hydrophobic interactions. Kwak et al. used this method to fabricate cytochrome c patterns on gold surfaces with a feature size of $200 \mathrm{~nm} .^{57}$ Because of the electron transport properties of cytochrome c, these protein patterns have potential applications in biomolecular photodiode systems. Lee et al. generated lysozyme, Immunoglobulin $\mathrm{G}(\mathrm{IgG})$ and retronectin arrays with feature sizes of $\sim$ 100-350 nm. ${ }^{60}$ The areas surrounding MHA patterns were passivated with 11-mercaptoundecyl-tri(ethylene glycol) $\left(\mathrm{EG}_{3}-\mathrm{SH}\right)$ instead of ODT. The biological activity of IgG arrays was retained as confirmed by binding anti-IgG to IgG. Patterns of retronectin, the cell adhesion recombinant fragment of fibronectin, were used to adhere to 3 T3 Swiss fibroblast cells. This array was used for a study of cellular interactions with nanopatterned surfaces. The authors observed that the cells only attached to the printed region and were immobilized in a more flattened morphology. ${ }^{60}$ Later, they extended this approach to fabrication of arrays of antibodies (anti-p24) with well-defined feature sizes of sub-100 nm. The bio-activity on $\mathrm{Au}$ surfaces in highly sensitive and selective immunoassays to detect human immunodeficiency virus type 1 p24 antigen (HIV-1 p24) was preserved (Figure 2.6). The HIV-1 p24 in plasma obtained from HIV-1-infected patients was hybridized with the antibody array. The bound protein was hybridized to a gold antibody-functionalized nanoparticle probe in order to amplify the detected signal. The nanoarray features in the three-component sandwich assay were confirmed by AFM. The authors demonstrated that such an immunoassay can exceed the detection limit of the conventional enzyme-linked ELISA based immunoassays by more than 1000 -fold. ${ }^{66}$ In these above mentioned cases, the biomolecular patterns were all fabricated on Au surfaces. Zhang et al. used the same concept to fabricate a single layer of Rabbit IgG dot patterns on MHA coated elevated $\mathrm{Au}$ nanostructures on $\mathrm{SiO}_{\mathrm{x}} / \mathrm{Si}$ substrates. The fluorophore-labeled anti rabbit IgG was used to confirm the bioactivity of proteins on solid supports. ${ }^{69}$ 


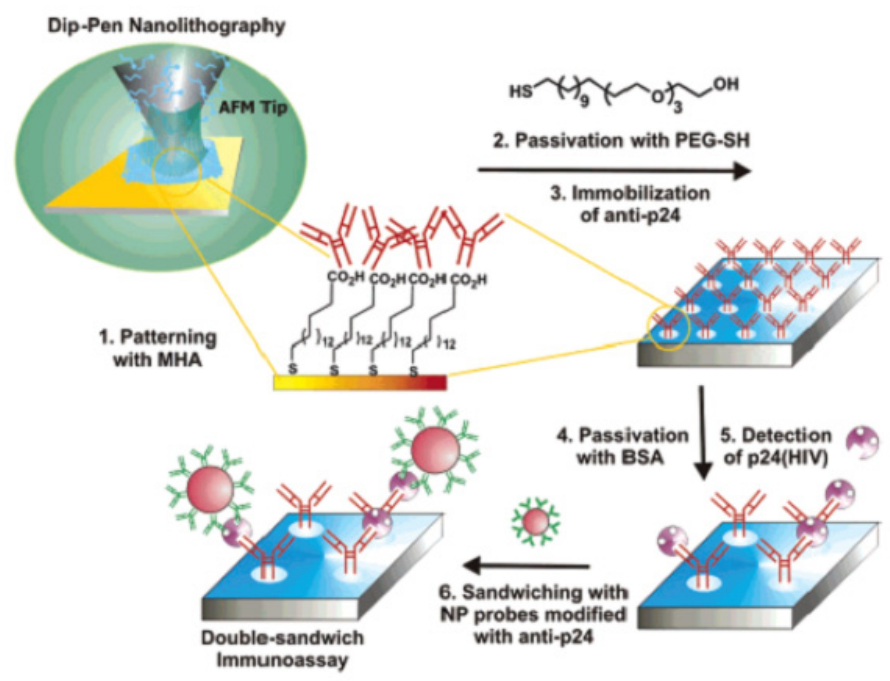

Figure 2.6. Schematic representation of the fabrication of immunoassay for detecting HIV-1 p24 antigen with anti-p24 antibody. Reprinted with permission from Ref. [66]. Copyright (2004) American Chemical Society.

Instead of utilizing electrostatic interactions, Demers et al. generated MHA patterns on $\mathrm{Au}$ surfaces to couple with alkylamine-modified DNA via formation of amide bonds. ${ }^{91}$ The non-patterned area was passivated with ODT. The authors found that the MHA patterns could be fabricated again on the areas previously passivated with ODT during the initial writing procedure. Therefore, another sequence of alkylamine-modified DNA could be immobilized onto the newly-created MHA patterns. The formation of two DNA patterns was confirmed by coupling DNA with complementary predesigned oligonucleotide-modified particles of different sizes. Different heights of DNA dot patterns and some stray particles nonspecifically bound to the areas surrounding the oligonucleotide patterns were observed in the AFM topography images. ${ }^{91}$

Smith et al. reported two ways to fabricate cowpea mosaic virus (CPMV) particles onto solid surfaces: one is via electrostatic/hydrophobic interaction and the other is via covalent attachment. ${ }^{55}$ In the non-covalent method, they immobilized CPMV particles onto DPN-generated MHA patterns with the rest of the area passivated with $\mathrm{EG}_{3}-\mathrm{SH}$. Since nonspecific adsorption of proteins often results in substantial denaturation and hence loss of biological activity, these authors developed a covalent method to immobilize 
CPMV particles. They wrote mixed ink, including 11-mercaptoundecyl-penta(ethylene glycol) disulfide and a mixed disulfide substituted with one maleimide, onto gold surfaces. Subsequently, cysteine mutant CPMV (Cys-CPMV) particles were attached to the patterned area via a covalent reaction of cysteine and maleimide. In both cases, AFM topography measurements confirmed the formation of single layer CPMV particles patterns. ${ }^{55}$ In the same year, Cheung et al. also demonstrated patterning Cys-CPMV particles with the same chemical reaction. They used DPN and nanografting to create amine-terminated alkanethiol molecules on gold surfaces. The amine groups were reacted subsequently with 3-maleimidopropionate N-hydroxysuccinimide ester (MPS) to form maleimide-terminated groups, to which Cys-CPMV particles were adhered. ${ }^{76}$

Membrane protein complexes attached to protonated amino-terminated SAMs created by DPN via electrostatic interactions have also been reported by Valiokas et al. ${ }^{65}$ The authors deposited $\mathrm{HS}\left(\mathrm{CH}_{2}\right)_{16} \mathrm{NH}_{2}$ onto a Au surface with a resolution of $\sim 300 \mathrm{~nm}$ and subsequently blocked the non-patterned region with $\mathrm{HS}(\mathrm{CH} 2)_{2} \mathrm{CONH}\left(\mathrm{CH}_{2} \mathrm{CH}_{2} \mathrm{O}\right)_{\sim 15} \mathrm{CH}_{3}$ for immobilization of the selective recruitment of membrane protein complexes from mutant Rhodobacter sphaeroides. These contain photosynthetic core complexes that form the reaction center of the light-harvesting complex one (LH1). ${ }^{65}$

Lee et al. demonstrated writing, with passive 26-probe arrays, of $N$-hydroxysuccinimide ester-terminated alkylthiols $\left(\mathrm{NHSC}_{11} \mathrm{SH}\right)$ onto $\mathrm{Au}$ surfaces as a template for immobilizing protein $\mathrm{A} / \mathrm{G} .^{71}$ The NHS ester groups are frequently used to couple with the primary amino groups of biomolecules via the formation of amide bonds. Protein $\mathrm{A} / \mathrm{G}$ has affinity to the Fc region of $\mathrm{IgG}$. By utilizing this affinity binding, massively parallel nanoarrays with biologically active antibodies can be generated. ${ }^{71}$ Hyun et al. also used NHS ester groups to fabricate biotin/streptavidin-terminated arrays. They first patterned MHA on Au surfaces with a feature size of $\sim 200 \mathrm{~nm}$. After obtaining the MHA patterns, they passivated the background with $\mathrm{EG}_{3}-\mathrm{SH}$ and then activated carboxylic acid terminated groups of an MHA SAM with $N$-hydroxysuccinimide (NHS) and 1-ethyl-3-(dimethylamino)propyl carbodiimide (EDAC) to form NHS ester groups. ${ }^{67}$ Biotinyl-3,6,9-trioxaundecanediamine (biotin- $\mathrm{NH}_{2}$ ) was used to generate biotin-terminated patterns. Subsequently, streptavidin, a tetrameric protein with very high affinity to bind four biotins $\left(\mathrm{K}_{\mathrm{a}} \sim 10^{13} \mathrm{M}^{-1}\right)$, was adhered to the patterned biotin area from solution. Therefore these protein arrays can be utilized to bind a variety of biotinylated molecules. The authors used biotinylated bovine serum albumin (Biotin-BSA) to demonstrate the retained functionality of this streptavidin-biotin system. ${ }^{67}$ Later, Hyun et 
al. extended their approach to immobilize amine-containing stimulus-responsive elastin-like polypeptide (ELP) onto patterned NHS ester-terminated regions. ${ }^{87}$ The authors believe that ELP is end-grafted at the surface in a highly orientated manner because ELP only has two amino groups, one located at the N-terminus and the other on the side chain of the sole lysine residue. Such ELP patterns can be used as templates to capture an ELP fusion protein (Trx-ELP) above its lower critical solution temperature (LCST). The dissociation of Trx-ELP occurs when proteins become soluble in aqueous solution. This switch process is reversible which offers the potential application of building reusable protein nanoarrays. $^{87}$

Rakickas et al. presented a platform to study protein-protein interactions and to form functional protein complexes at nanometer scale. For this purpose, multivalent chelator (MCh)-terminated templates were fabricated in a stepwise procedure combining dip-pen nanolithography and molecular recognition-directed assembly (Figure 2.7). The biotin-oligo(ethylene glycol) (OEG) disulfide molecules were first deposited on gold with line widths below $100 \mathrm{~nm}$ and then streptavidin was adhered to the biotin-terminated patterns from solution. Subsequently, the streptavidin groups were attached with tris-nitriloacetic acid biotin derivatives $\left({ }^{\mathrm{BT}}\right.$ trisNTA) to form $\mathrm{MCh}$ terminated templates for assembly of histidine-tagged (His-tagged) proteins. Histidine tags are generally engineered onto protein sequences to facilitate protein purification in analytical procedures. The ${ }^{\mathrm{BT}}$ trisNTA coordinated with $\mathrm{Ni}(\mathrm{II})$ area was used to immobilize His $_{10}$-ifnar2 proteins, whose functionality was confirmed by coupling INF $\alpha 2$ labeled with $\mathrm{QD}_{655}$. This process was reversible by adding imidazole solution to remove His-tagged proteins. ${ }^{79}$ This His-Ni(II)-NTA system is frequently used to immobilize biomolecules with orientation on solid surfaces. ${ }^{93}$

Hoover et al. presented an indirect approach to produce peptide arrays for immobilizing cells (3T3Swiss Albino mouse fibroblasts). They produced nanoarrays of hydroquinone terminated alkanethiol on glass substrates covered by a thin gold film. The non-patterned regions were passivated with tetra(ethylene glycol) terminated alkanethiol. By electrochemically oxidizing the nanopatterned hydroquinone, the corresponding quinone was obtained, which can react selectively with water soluble oxyamine-terminated linear Arg-Gly-Asp (RGD) peptide. These peptide patterns were used to study the polarity of adhered cells without the influences of cell-cell interactions. It was found that a cell was affected by its nano-environment to polarize toward the patterned higher density peptide region. ${ }^{83}$ 


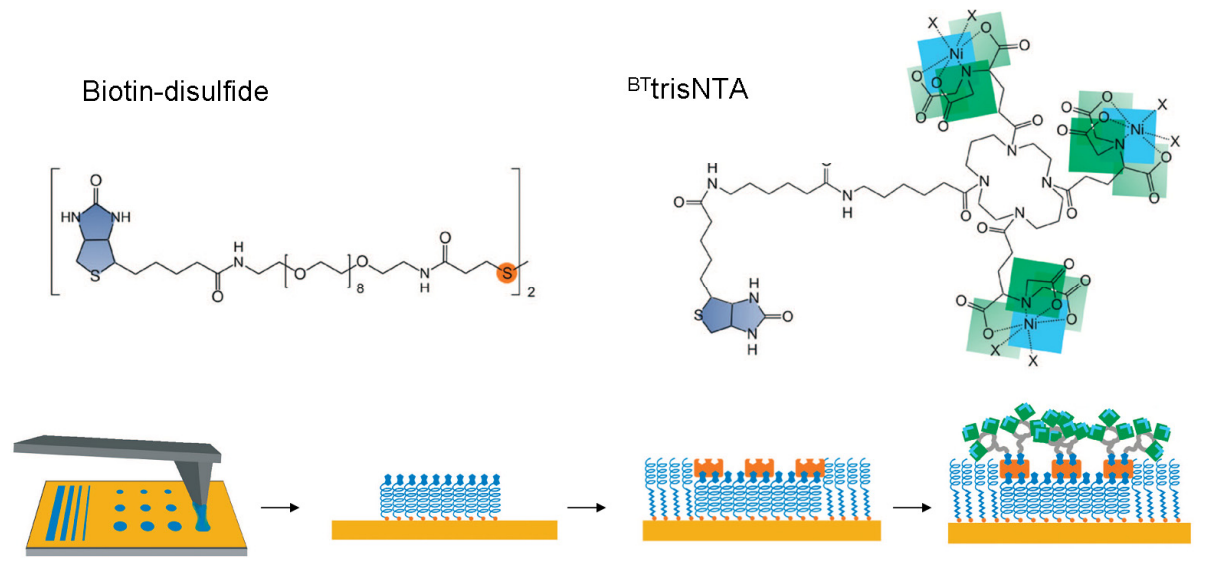

Figure 2.7. Schematic representation of the fabrication of ${ }^{B T}$ trisNTA-terminated nanoarrays by DPN used for studying protein-protein interactions. Reprinted with permission from Ref. [79]. Copyright (2008) American Chemical Society.

Vega et al. relied on the ability of $\mathrm{Zn}$ (II) ions to bridge a surface patterned with MHA and the tobacco mosaic virus (TMV) with its carboxylate-rich surface. They demonstrated the possibility of isolating and controlling the orientation of TMV particles. ${ }^{70}$ Later, they used the same approach to immobilize antibodies, such as IgY and IgM. Although they did not know the precise chemistry behind the reaction between the antibodies and $\mathrm{Zn}$ (II) ions, this approach enables the further adhesion of protein $A, G, A / G$ or $L$ with functionality. ${ }^{72}$

\subsubsection{Silicon-based surfaces}

The majority of DPN experiments use the thiol-on-gold couple, where the chemisorption of the thiol molecules onto gold surfaces is the driving force of ink transfer from tips. Patterning of biomolecules with indirect DPN on silicon-based surfaces is also reported. ${ }^{23,68,74,75,78,81,90,97}$

Pena et al. reported one way to fabricate biotin-terminated arrays to immobilize fluorophore-labeled neutravidin on silicon based substrates. ${ }^{68}$ They first sputtered polyethylenimine (PEI) onto $\mathrm{SiO}_{2}$ surfaces, followed by depositing NHS functionalized biotin. Some nonspecific binding was observed, but the authors claimed that the signal to 
noise ratio was sufficient. Nyamjav et al. fabricated poly(allylamine hydrochloride) $(\mathrm{PAH})$, a polyelectrolyte, patterns on $\mathrm{SiO}_{\mathrm{x}}$ wafers, demonstrating that these well-defined positively charged regions can be used to guide the stretching of long DNA molecules. ${ }^{90}$ Such stretched DNA adhered to patterned PAH areas and was subsequently used as a template to immobilize $\mathrm{Fe}_{3} \mathrm{O}_{4}$ nanoparticles by electrostatic interactions. ${ }^{92}$

Jung et al. demonstrated that it is possible to fabricate alkoxysilanes patterns on glass substrates. They used 3 '-mercaptopropyltrimethoxysilane (MPTMS) as the ink molecules. The MPTPS patterns were verified by reaction with biotin-maleimide via the formation of thioether bonds to immobilize Cy3-labeled streptavidin. ${ }^{97}$ Later, the authors extended this approach to fabricate (+)-biotinyl-3-maleimidopropionamidyl-3,6-dioxaoctanediamine (maleimide $\mathrm{PEO}_{2}$-biotin) directly onto MPTPS-functionalized glass substrates. ${ }^{23}$ However, the authors observed that obtaining consistent patterns of the maleimide- $\mathrm{PEO}_{2}$-biotin was very difficult even when DPN experiments were performed with RH above $90 \%$. Finally, they found that adding small amounts of the nonionic surfactant Tween-20 (polyoxyethylene sorbitan monolaurate) to the ink can activate the transfer of the biotin linker molecules from the tip to the MPTMS-coated substrates, enabling biotin patterning routinely with sub-100 $\mathrm{nm}$ resolution at a moderate relative humidity of $50-65 \% \mathrm{RH}$. Cy3-labeled streptavidin was again used to confirm the successful generation of maleimide $\mathrm{PEO}_{2}$-biotin patterns. ${ }^{23}$ The same chemical strategy has also been utilized by Kim et $a .^{74}$ They wrote maleimido- $\mathrm{C}_{3}$-nitrilotriacetic acid (NTA) onto MPTMS-functionalized glass substrates for the effective immobilization of His-tagged enhanced green fluorescent proteins by utilizing coordination chemistry. ${ }^{74}$ Sekula et al. demonstrated spatial patterned supported phospholipid membranes with biotin and nitrilotriacetic acid (NTA) head groups onto hydrophilic glass substrates as templates to immobilize Cy3-labeled streptavidin and His-tagged green fluorescent proteins (His-GFP) (Figure 2.8a), respectively. By using passive 26-probes arrays, integrated protein nanoarrays exploiting two different funtionalities were generated (Figure 2.8b). The authors also demonstrated that these biomimetic membrane patterns can be used as substrates for cell culture, by the selective adhesion and activation of T-cells. ${ }^{75}$

Zhou et al. reported an approach to fabricate nanopatterns of peptides of specific chain length. The preparation procedure started by depositing $\mathrm{G} 4-\mathrm{NH}_{2}$ polyamidoamine (PAMAM) dendrimers onto $\mathrm{Si} / \mathrm{SiOx}$ substrates with feature sizes of $\sim 180 \mathrm{~nm}$ via electrostatic interactions. The patterned dendrimer substrates were incubated in tryptophan- $N$-carboxyanhydride (Trp-NCA) solution for different times to initiate 
ring-opening polymerization (ROP), a well known method to obtain oligopeptides with variable chain lengths. ${ }^{81}$

a)

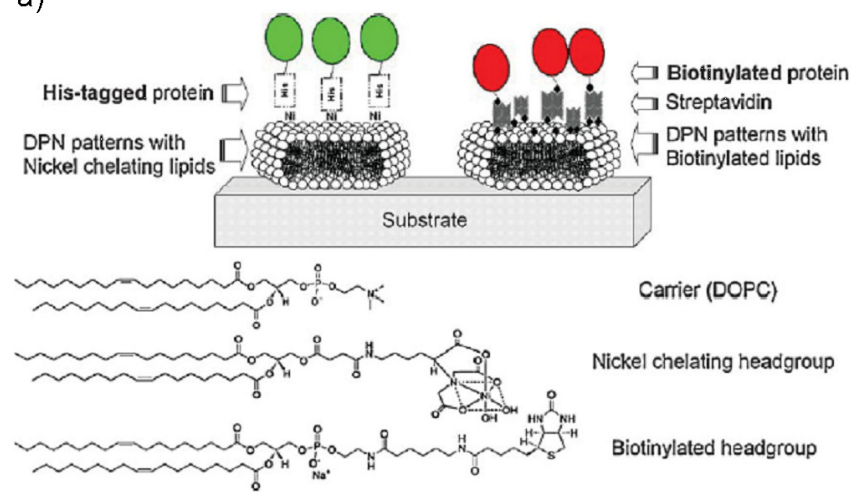

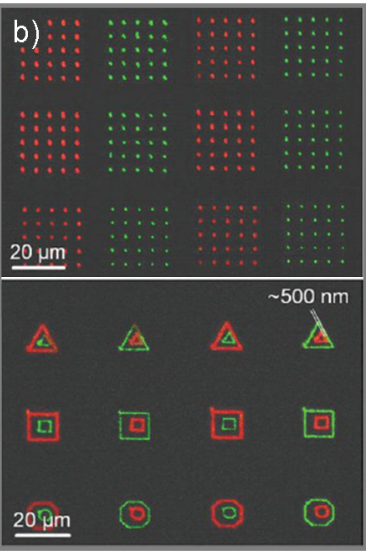

Figure 2.8. (a) Schematic representation of the protein-coupling strategies by pattering lipids onto a substrate and the chemical structures of the used lipids. (b) Fluorescence image of Cy3-labeled streptavidin and His-GFP selectively immobilized on the pre-generated different lipid patterns written with a passive 26-probes array. Reproduced with permission from Ref. [75]. Copyright (2008) Wiley-VCH Verlag GmbH \& Co. KGaA.

\subsubsection{Direct DPN Methods}

Compared with indirect DPN, direct DPN is a simple and straightforward approach to fabricate protein patterns without non-specific binding. ${ }^{2}$ However, it has several restrictions, such as, slow transportation of high molecular weight biomolecules from an AFM tip to a substrate, difficulties of preserving the biological functionalities of biomolecules on the tips surface during writing, and short operation time due to the limited ink volume. ${ }^{64}$ To overcome these restrictions, chemical modification of AFM tip surfaces is a common strategy used to overcome the restrictions mentioned above. Therefore, in this section we separately address tips with and without surface functionalization. 


\subsubsection{With bare tips}

\subsubsection{1 a Gold surfaces}

Fabricating nanostructures with DPN is usually performed by operating AFM in contact mode. However, in the bio-related domain, the use of tapping-mode AFM for lithography and imaging may be more suitable, because of the sensitivity of biomolecules to the shear and friction forces required to control the surface in the contact mode. Two examples of utilizing tapping-mode AFM to deposit biomolecules were reported. The first was reported by Wilson et al. They delivered thiolated collagen and collagen-like peptides onto gold substrates with line widths as small as 30-50 nm and showed that collagen still preserved its native triple-helical structure and bioactivity. ${ }^{62}$ Later, Agarwal et al. used DPN in tapping mode to deposit synthetic peptides (MH2) containing an active cysteine (Cys) residue onto a gold surface with a feature size as small as $70 \mathrm{~nm}^{84}$

Cho et al. demonstrated that thiol-containing TAT peptides, derived from the TAT protein of the HIV-1, can be directly written onto Au substrates. ${ }^{88}$ More recently, Wei et al. generated 3-repeat GBP1 peptide with biotin incorporated (biotin-3RGBP1) onto Au surfaces with the smallest feature size of $\sim 80 \mathrm{~nm}$. Alexa Fluor 594-labeled streptavidin was subsequently used to visualize the biotinylated peptide patterns. ${ }^{80}$ Both Cho et al. and Wei et al. also demonstrated peptide patterns on Si-based surfaces, which are described in the next section.

\subsubsection{1b Silicon-based surfaces}

Noy et al. patterned lines of human chorionic gonadotropin (HCG) antibody tagged with tetramethylrhodamine (TMR) at sub-micrometer scale on a glass substrate pretreated with 3-glycidoxypropyltrimethoxysilane via the reaction of amines with epoxides. The epoxy-terminated monolayer facilitates the adhesion of proteins to surfaces. The resulting patterns were examined by an instrument that combines AFM and scanning confocal microscopy. ${ }^{61}$ However, the biological activity of the resulting patterns was not demonstrated.

A ribosome display is a cell-free system for in vitro selection of proteins and peptides from large libraries that can produce protein-ribosome-mRNA fusion molecules by coupling individual proteins (phenotypes) to their corresponding mRNA (genotypes). Kim et al. demonstrated deposition of acrylamide-modified oligonucleotides mixed with deionized water and $N, N$-dimethylformamide (DMF) onto MPTMS-coated glass 
substrates. The volume percentage of DMF used to facilitate the ink transport was very important for obtaining consistent patterns. The bioactivity of written oligonucleotide was confirmed via hybridization with fluorophore-labeled complementary oligonucleotide or protein-ribosome-mRNA fusion molecules. The authors demonstrated that this approach (Figure 2.9) can be used to fabricate protein arrays without prior protein purification. ${ }^{73}$

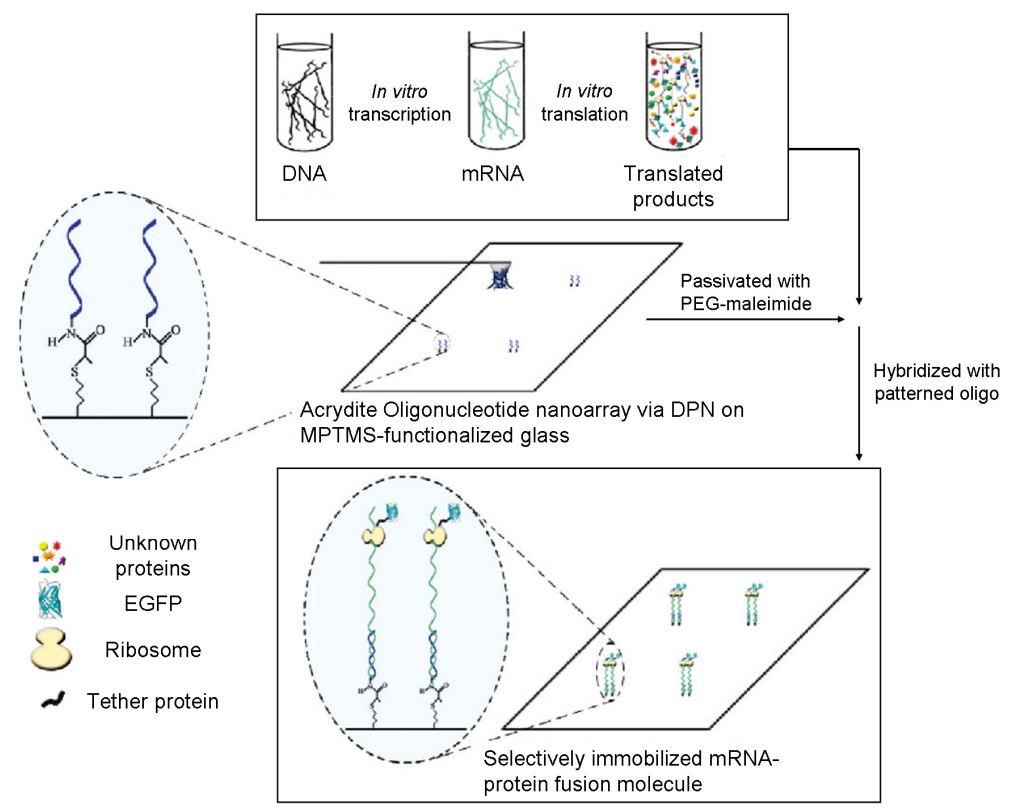

Figure 2.9. Schematic illustration of the ribosome display technique and the procedure of fabricating protein arrays by directly depositing oligonucleotide as templates. Reproduced with permission from Ref. [73]. Copyright (2008) Wiley-VCH Verlag GmbH \& Co. KGaA.

Cho et al. not only demonstrated direct patterning of thiol-containing TAT peptides onto an $\mathrm{Au}$ surface but also onto $\mathrm{Si}$ substrates functionalized with a malemide-terminated monolayer. ${ }^{85}$ For patterning TAT peptides on surface modified Si substrates, $\sim 80 \% \mathrm{RH}$ was required in order to obtain nanostructures. This was attributed to the high hydrophobicity of the malemide-terminated monolayer. ${ }^{85}$ Besides fabricating biotin-3RGBP1 peptide patterns on Au surfaces, Wei et al. also deposited two kinds of 
biotinylated peptide (biotin-QBP1 and biotin-QBP3) onto Si surfaces with the smallest feature size of $\sim 80 \mathrm{~nm}$ and confirmed the formation of the peptide patterns with Alexa Fluor 594-labled streptavidin. ${ }^{80}$ Sistiabudi et al. investigated how surface properties affected the direct writing of polyarginine and poly(glutamic acid) polypeptides. Three different surfaces were utilized including hydrophilic bare silicon oxide and 3-aminopropyltriethoxysilane (APTES)-functionalized $\mathrm{Si}$ substrate and hydrophobic trimethoxypropylsilane (TMPS)-functionalized Si substrate. Their results demonstrated that both polypeptides were not transferred onto hydrophobic TMPS-functionalized surface even when performing DPN experiments under high RH conditions. Besides, they found that the transport of polypeptides was facilitated by adding Tween-20 surfactant, because of the increased wettability of the collagenous surface. The deposition of ink was therefore able to be operated at lower contact force and $\mathrm{RH}^{89}$

\subsubsection{1c Other surfaces}

Most DPN experiments have been performed by writing ink onto hard solid supports and it is a challenge to deliver molecules onto soft matter and therefore only few examples have been reported. Li et al. wrote Cy3-antibody and Cy3-streptavidin onto a stretched DNA, which was pre-deposited onto a 3-aminopropyltriethoxysilane (APTES)-functionalized mica surface. The smallest feature size achieved was $\sim 40 \mathrm{~nm} .^{77}$ Sistiabudi et al. not only deposited polyarginine and poly(glutamic acid) polypeptide onto Si-based substrates but also onto the inner collagenous layer of Brunch's membrane (ICLBM). More recently, they presented the deposition of biotin-collagen-binding peptide (CBP) molecules onto the ICLBM surface and verified their bioactivity through the binding of Cy3-labeled streptavidin. ${ }^{82}$

\subsubsection{With functionalized tips}

\subsubsection{2a Gold surfaces}

Lee et al. reported a method to functionalize Au-coated $\mathrm{Si}_{3} \mathrm{~N}_{4}$ cantilevers, which was used to fabricate lysozyme (Lyz) and rabbit IgG arrays at the same time with the smallest feature size of $\sim 45 \mathrm{~nm}$ in diameter without cross-contaminations. ${ }^{30}$ The process started with growing a monolayer of symmetric 11-mercapto-undecylpenta(ethylene glycol)disulfide (PEG) on a cantilever, coating of an Au layer on the tip side via thermal 
evaporation, and finally forming a carboxylic-terminated SAM on the tip surface. The PEG layer prevents the adsorption of protein on the backside of the cantilever and the hydrophilic carboxylic groups can facilitate the adsorption of proteins onto the tip surfaces. The Cys residues of rabbit IgG also provided a strong driving force to adhere proteins onto gold surfaces due to thiol-Au reaction. The bio-recognition properties of two component protein arrays were confirmed by the observation of anti-rabbit IgG binding to the rabbit IgG features only (Figure 2.10a). ${ }^{30}$ The authors found that the optimum patterning results were obtained when performing DPN at a $\mathrm{RH}$ of $\sim 80-90 \%$ at room temperature. $^{30}$

Another possibility to increase the hydrophilic property of the tip surface by functionalizing the tip with mercaptoundecanoic acid (MUDA) has been reported. ${ }^{56}$ Angiogenin and integrin $\alpha_{v} \beta_{3}$ were written on a gold substrate coated with a layer of Prolinker ${ }^{\mathrm{TM}}$ with pattern sizes as small as $120 \mathrm{~nm}$. The Prolinker ${ }^{\mathrm{TM}}$ contains crown ether moieties that act as a host cavity for protonated amino groups on protein surfaces. ${ }^{56}$

Demers et al. found that it was difficult to control patterned DNA sizes and shapes while using a bare $\mathrm{Si}_{3} \mathrm{~N}_{4}$ tip. Therefore, they functionalized tip surfaces with 3 '-aminopropyltrimethoxysilane (APTMS) to improve the adhesion of DNA to the tips. ${ }^{29}$ Using these APTMS-functionalized tips, DNA bearing hexanethiol groups and oligonucleotides with 5'-terminal acrylamide groups were written onto gold at sub-100 nm scale. $^{29}$

\subsubsection{2b Silicon-based surfaces}

Demers et al. again used APTMS-functionalized AFM tips to write oligonucleotides containing 5'-terminal acrylamide groups onto MPTPS-modified $\mathrm{SiO}_{2}$ substrates at sub-100 nm scale. $^{29}$ The authors demonstrated the possibility of generating two-component DNA arrays on $\mathrm{SiO}_{2}$ surfaces while retaining their biological activities, which was confirmed by hybridization with complementary fluorophore-labeled DNA (Figure 2.10b). ${ }^{29}$

Lim et al. modified an AFM tip with 2-[methoxypoly(ethyleneoxy)-propyl] trimethoxysilane (Si-PEG) to write (Anti-)rabbit IgG onto a negatively charged, aldehyde-terminated $\mathrm{SiO}_{2}$ surface via respectively electrostatic interaction and covalent bonding down to a length scale of $\sim 50 \mathrm{~nm} .{ }^{59} \mathrm{Si}-\mathrm{PEG}$ can facilitate the transfer of proteins from tip to surfaces and also prevent the denaturation of proteins on the tip surface. ${ }^{59}$ 
Jiang et al. demonstrated direct patterning of two kinds of amphiphile (PA) peptide nanofibers onto $\mathrm{Si}$ substrates at micrometer scale by using AFM tips coated with poly(ethylene glycol) (PEG), which avoids the self-assembly of nanofibers on the tip surfaces. $^{86}$
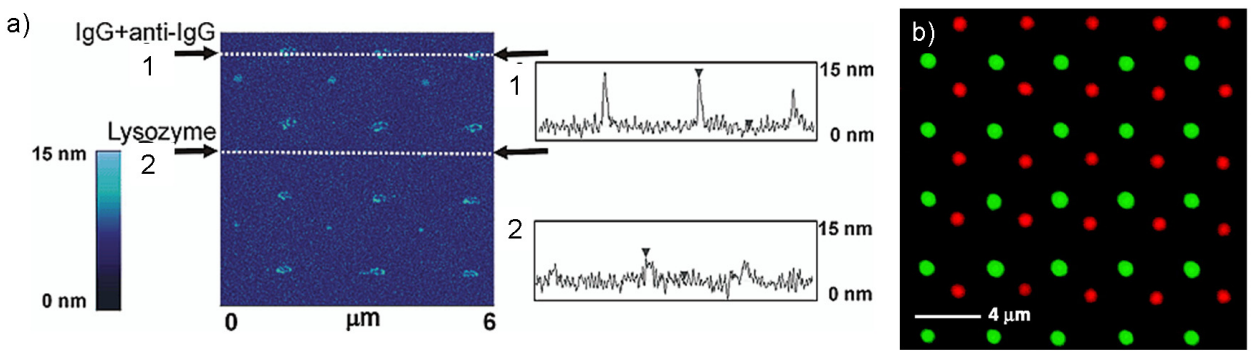

Figure 2.10. (a) AFM topography image of two-components protein nanoarrays after reacting with anti-IgG. The height increase was only observed on the the IgG features indicating that the bio-recognition properties of $\operatorname{Ig} G$ were preserved. Reprinted with permission from Ref. [30]. Copyright (2003) American Chemical Society. (b) Combined red-green epifluorescence image of two-components DNA nanoarrays after simultaneously hybridizing with two different complementary fluorophore-labeled DNA. Reprinted with permission from Ref. [29]. Copyright (2003) AAAS.

Apart from microfluidic approaches, porous matrix materials have been developed to functionalize the AFM tip surfaces to increase the total amount of coated ink molecules. A few methods have been reported. Choi et al. fabricated hydrophilic nanoporous polyoxazoline-coated $\mathrm{Si}$ AFM tips by functionalizing the bare surface with 11-iodoundecyltrichlorosilane as an initiator and performing the polymerization of 2-methyl-2-oxazoline monomers on the pre-treated surface. ${ }^{64}$ Human $\operatorname{IgG}$ and prostate specific antigen (PSA) protein arrays were generated onto $\mathrm{N}$-2-amino-ethyl-11aminoundecyltrimethoxysilane (AUMS)-functionalized Si substrate with feature sizes as small as $\sim 250 \mathrm{~nm}$ and $\sim 85 \mathrm{~nm}$ in diameter, respectively. The authors demonstrated that it is at least five times faster to generate protein nanoarrays with the nanoporous polyoxazoline-coated tip than with a conventional Si tip. ${ }^{64}$ By using agarose as a "universal" carrier to generate biomolecular patterns, Senesi et al. presented another way 
(Figure 2.11a and b) to encapsulate proteins (IgG) and oligonucleotides onto AFM tip surfaces. They wrote the smallest feature size of $\sim 50 \mathrm{~nm}$ on $N$-hydroxysuccinimide-ester activated glass substrates. By reacting with fluorophore-labeled anti-IgG and complementary oligonucleotides, respectively, the biorecognition properties of $\operatorname{IgG}$ and oligonucleotides were confirmed. Combined with passive probe arrays, Cy3-labeled oligonucleotide arrays were fabricated in a parallel manner (Figure 2.11c). The authors reported that the process of agarose-assisted DPN for patterning biomolecules is very fast and can be systematically adjusted by controlling the concentrations of accelerating agents, e.g. tricine buffer, used in agarose matrix. ${ }^{24}$

a)

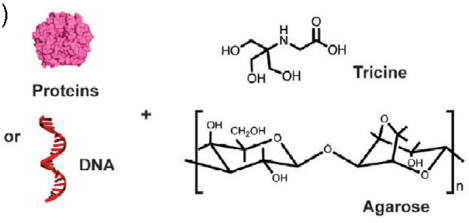

c)

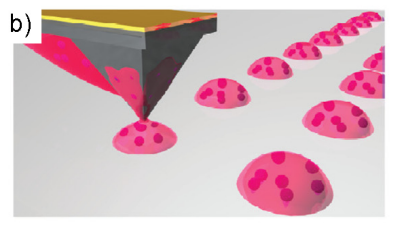

10 inim

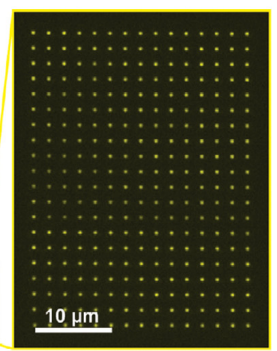

Figure 2.11. (a) Schematic representation of ink and matrix components; (b) an illustration showing the process of agarose-assisted DPN; (c) epifluorescent microscope image of a $15 \times 20$ array of $500 \mathrm{~nm}$ Cy3 labeled oligonucleotide features generated in parallel from a 12-tip cantilever array. Reprinted with permission from Ref. [24]. Copyright (2009) American Chemical Society.

\subsubsection{2c Other surfaces}

Most direct DPN experiments have been performed by writing ink onto either $\mathrm{Au}$ or Si-based substrates. There is one example of using other surfaces reported by Nam et al.. They described a way to facilitate the wetting and transport of His-tagged ubiquitin or thioredoxin by coating a thin layer of $\sim 5 \mathrm{~nm}$ nickel onto a $\mathrm{Si}_{3} \mathrm{~N}_{4}$ AFM tip. These proteins were deposited onto nickel oxide surfaces with a feature size down to $\sim 85 \mathrm{~nm} .{ }^{58}$ 


\subsection{Other AFM-based lithography techniques for patterning biomolecules}

Besides DPN, there are a few other AFM-based lithography techniques for generating biomolecule patterns including nanoshaving/nanografting, enzymatic lithography, bias-AFM lithography, and fountain pen lithography. ${ }^{13}$

Figure 2.12a illustrates the operation procedures for indirect and direct nanografting. The concept is to shave SAM molecules by force with an AFM tip and then refill from solution the gaps with desired molecules that have higher affinity to the surface than the removed molecules. ${ }^{98}$ In indirect nanografting, it was reported that carboxylic acid-terminated SAMs or aldhyde-terminated SAMs were nanografted onto methyl-terminated SAMs on Au surfaces for immobilizing Lysozyme and IgG onto the patterned region with a feature size as small as $10 \mathrm{~nm}$, either via electrostatic interaction or formation of imine bonds, respectively. ${ }^{99,} 100$ By using 1-ethyl-3-(3-dimethylaminopropyl)-carbodiimide (EDC), IgG was also bound to carboxylic acid groups via the formation of amide bonds. ${ }^{101}$ Cheung et al. used indirect DPN to pattern Cys-CPMV onto maleimide-terminated monolayers. ${ }^{76} \mathrm{~A}$ few years later, they demonstrated nanografting of Ni-NTA alkanethiol molecules onto PEG-terminated alkanethiol for immobilizing His-tagged CPMV with a line width equal to the virus diameter $(\sim \quad 28 \mathrm{~nm}) .{ }^{102}$ Lee et al. demonstrated nanografting of octyldimethylmonochlorosilane $\left(\mathrm{C}_{8} \mathrm{DMS}\right)$ with aminosilane on a silicon oxide surface. The aminosilane area created was subsequently used to immobilize negatively charged DNA via electrostatic interactions. ${ }^{103}$

Direct nanografting is in most of the cases based on immobilizing DNA ${ }^{104}$ and proteins $^{105,106}$ containing thiol groups onto Au surfaces via the well-known Au-thiol interaction. An extended approach of nanografting called native protein nanolithography was reported by Tinazli et al. to assemble proteins or multiprotein complexes at nanometer scale under native conditions. Their concept (Figure 2.12b) uses the contact oscillation mode of AFM to detach one kind of His-tagged proteins and replace them by another kind on a Ni-NTA-terminated monolayer. Functional protein complexes, with uniform orientation at dimensions down to $50 \mathrm{~nm}$, were obtained (Figure 2.12c). ${ }^{107}$ The disadvantage of nanografting is that the type of surface is limited to Au. However, this technique enables generating high resolution nanostructures with a feature size as small as $10 \mathrm{~nm}^{100}$ 

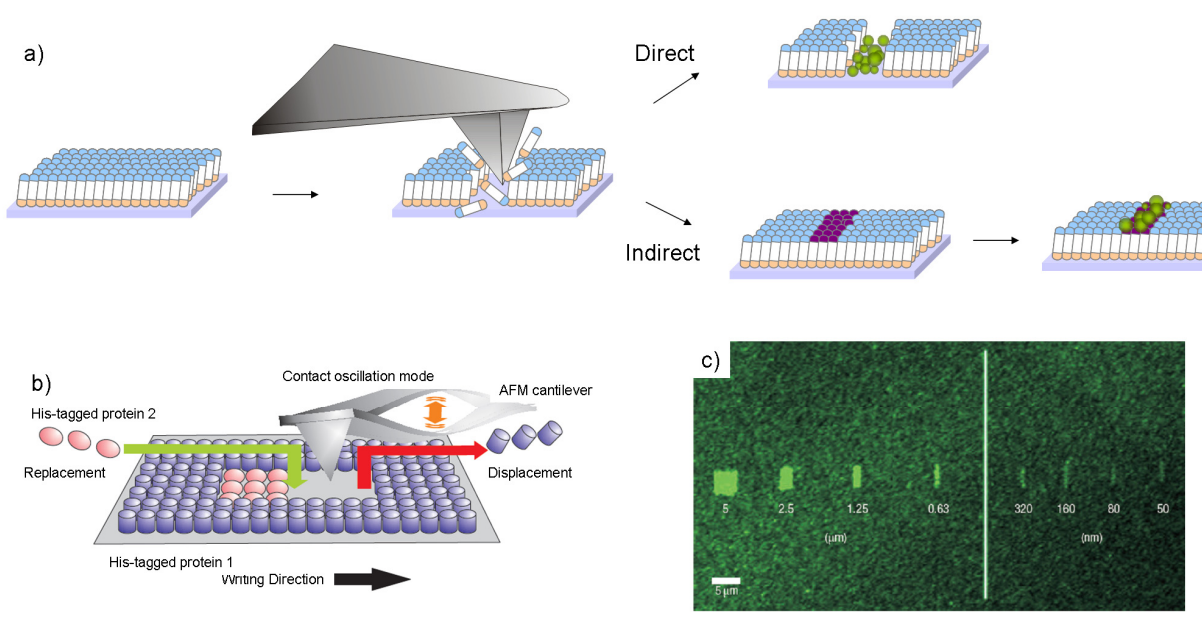

Figure 2.12. Schematic representation of (a) direct and indirect nanografting technique for patterning biomolecules, (b) native protein lithography. (c)Fluorescent image of protein patterns generated by local exchange of affinity-captured proteasome complexes $\left(\alpha \mathrm{N}-\mathrm{His}_{6}\right)$ with Oregon Green-labeled MBP-His 10 . Reprinted by permission from Macmillan Publishers Ltd: [Nature Nanotechnology, Ref. 107], copyright (2007).

The idea of enzymatic lithography is to bring an enzyme-coated tip to touch a surface and then induce an enzymatic reaction. Takeda et al. first demonstrated this technique by using a Staphylococcal serine V8 protease-coated AFM tip to digest peptide functionalized mica on a $\mu \mathrm{m}$ scale surface in a buffered solution. ${ }^{108}$ Later, the authors utilized resonance energy transfer (FRET) peptides to confirm the reaction of enzymatic lithography. A quencher residue was released from the peptide when Staphylococcal serine V8 protease digested the reporter peptide. This resulted in the enhancement of fluorescence intensity. ${ }^{109}$ Hyun et al. proposed another approach by scanning oligonucleotide-functionalized Au surfaces with a DNase I-coated tip. The DNase I was activated by adding $\mathrm{Mg}$ (II) ions in the solution and then the local digestion of oligonucleotides was carried out to form nanotrenches on surfaces. ${ }^{110}$

Tang et al. presented another approach to deliver proteins with nanoscale precision by functionalizing tip surfaces with a streptavidin-terminated photocleavable cross linker. By irradiating with UV light, the streptavidin molecules were released from the tip surface and bound well to the biotin-terminated monolayer. ${ }^{111}$ 
Several groups demonstrated that applying a bias between tip and substrate locally modifies the surface properties ${ }^{12-115}$ for adhering biomolecules or facilitating the transport of biomolecules. ${ }^{116}$ Pavlovic et al. converted a thiol-terminated monolayer to a thiolsulfinates/thiolsulfonates-terminated monolayer by applying a voltage. Thiol-containing proteins were immobilized onto the oxidized area via the formation of disulfide bonds. The advantage of this approach is that the bonding can be reversed by adding dithiothreithol (DTT) to break the disulfide bonds. ${ }^{115} \mathrm{Gu}$ et al. applied a voltage on oligo(ethylene glycol) (OEG)-terminated substrates to generate dot patterns with a diameter of $\sim 90 \mathrm{~nm}$. Their results suggested that the bias-applied hydroxyl groups could be converted to carboxylic groups by adding succinic anhydride. Subsequently, avidin was attached to the patterned region in the presence of 1-ethyl-3-(3-dimethylaminopropyl)carbodiimide (EDAC) and biotinylated-BSA was used to confirm the success of the reaction. ${ }^{114}$ Recently, the same group demonstrated that the resolution of feature size can be scaled down to sub-10 nm. ${ }^{117}$

The disadvantages of DPN for patterning biomolecules are the short operation times due to the limited ink volume and difficulties of maintaining biological activity on dry tip surfaces. Therefore, cantilevers connected to an ink reservoir were developed to overcome these restrictions. Taha et al. used cantilevered nanopipette fountain pens to deliver protein G and GFP onto super-aldehyde surfaces and BSA-coated substrates respectively. The smallest feature size was $\sim 200 \mathrm{~nm} \cdot{ }^{118} \mathrm{Xu}$ et al. developed quill-type surface patterning chips including both single and multiple cantilevers which were used to generate Cy3-streptavidin arrays with routine spot sizes of 2-3 $\mu \mathrm{m}$ onto dithiobis(succinimidyl) undecanoate monolayer-functionalized $\mathrm{Au}$ substrates. ${ }^{119}$ Moldovan et al. designed so called nanofountain pen (NFP) arrays ${ }^{120-122}$ with the ability of carrying multiple inks (Figure 2.13a and 2.13b). Several other examples of generating biomolecular features with these nanofountain pen arrays were reported by Espinosa's group. $^{122-124}$ For example, Kim et al. utilized NFP to deposit alkanethiol-modified oligonucleotides onto $\mathrm{Au}$ surfaces with smallest feature sizes of $\sim 200 \mathrm{~nm}$ and subsequently passivated the unpatterned area with hexanethiol to avoid unspecific binding. The formation of oligonucleotide patterns was confirmed by hybridization of the linker and probe DNA strands (Figure 2.13c). ${ }^{122}$ 
a)

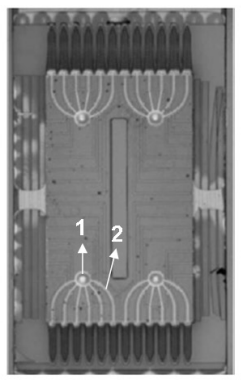

b)

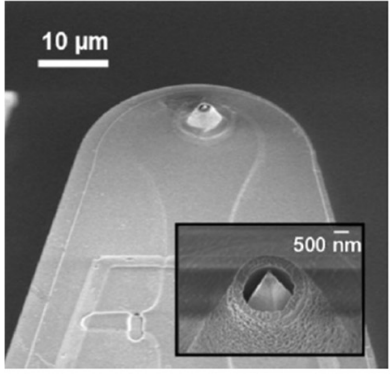

c)

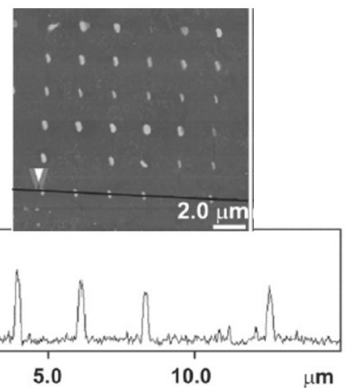

Figure 2.13. (a) Optical image of the second generation nanofountain pen (NFP) chip. 1 and 2 indicate on-chip reservoir and microchannel, respectively. (b) SEM image of a cantilever integrated with a volcano tip at the end; in the inset a close-up of the volcano tip is shown. (c) AFM tapping-mode topography image of an array of dots generated with three different tip-surface contact times of 2, 1, and $0.5 \mathrm{~s}$, for each alternating row of the array. The height profile of the same array indicated by the black line is shown below of the AFM image. Reproduced with permission from Ref. [122]. Copyright (2008) Wiley-VCH Verlag GmbH \& Co. KGaA..

\subsection{Conclusions}

In summary, in the first part of this chapter, recent developments of dip-pen nanolithography (DPN), which is a relatively inexpensive and high-resolution AFM-based lithography technique, are reviewed. Although the patterning efficiency of DPN was low during the early stages of development of the technique, the successful design and fabrication of active and passive probe arrays during the past few years has dramatically improved the patterning efficiency. These probe arrays allow users to deposit different ink molecules simultaneously and in a massively parallel manner at sub-micrometer scale.

In the second part of this chapter, different chemical strategies used to fabricate biomolecular nanostructures on solid supports with either direct or indirect DPN are reviewed. It can be concluded that gold and Si-based materials are the most commonly used solid substrates for DPN. Also, chemical modification of AFM tip surfaces is a frequently utilized strategy for direct DPN to facilitate the deposition of biomolecules onto surfaces. In addition to DPN, a selection of AFM-based lithography techniques used for bio-patterning was briefly described. 
Besides the strategies reviewed in this chapter, an indirect DPN approach (see also Chapter 4) and a direct DPN approach (see also Chapter 6) are presented in this thesis to fabricate protein patterns. Chapter 4 describes how $\mathrm{Ni}(\mathrm{II})$ ions can be written onto NTA-terminated monolayer-functionalized glass substrates as templates to immobilize His-tagged visible fluorescent proteins (His-VFPs) via a specific metal-protein interaction. This approach not only enables the generation of protein patterns but also the characterization of the dependence of $\mathrm{Ni}$ (II) ions transfer on fundamental parameters of DPN, such as writing speeds and tip-surface contact times. In Chapter 6, direct depositions of His-VFPs onto Ni(II)-NTA monolayers will be described. In order to absorb larger amount of biomolecules onto tip surfaces, a method is developed to coat the surfaces of AFM tips with a thin layer of porous film by using Layer-by-Layer (LbL) techniques. Compared to a bare AFM tip, this porous film can act as a larger volume ink reservoir for long-term DPN writing.

The combination of nanofabrication and biomolecules enables the study of fundamental interactions between cells and nano-structured surfaces, and the development of high-density bio-nanoarrays with potential for several applications including fast screening of routine diagnostic tests.

\subsection{References}

1. Mendes, P. M.; Yeung, C. L.; Preece, J. A. Bio-Nanopatterning of Surfaces. Nanoscale Res. Lett. 2007, 2 , 373-384.

2. Christman, K. L.; Enriquez-Rios, V. D.; Maynard, H. D. Nanopatterning Proteins and Peptides. Soft Matter 2006, 2, 928-939.

3. Lom, B.; Healy, K. E.; Hockberger, P. E. A Versatile Technique for Patterning Biomolecules onto Glass Coverslips. J. Neurosci. Meth. 1993, 50, 385-397.

4. Schwarz, A.; Rossier, J. S.; Roulet, E.; Mermod, N.; Roberts, M. A.; Girault, H. H. Micropatterning of Biomolecules on Polymer Substrates. Langmuir 1998, 14, 5526-5531.

5. Rozkiewicz, D. I.; Gierlich, J.; Burley, G. A.; Gutsmiedl, K.; Carell, T.; Ravoo, B. J.; Reinhoudt, D. N. Transfer Printing of DNA by "Click" Chemistry. Chembiochem 2007, 8, 1997-2002.

6. Rozkiewicz, D. I.; Brugman, W.; Kerkhoven, R. M.; Ravoo, B. J.; Reinhoudt, D. N. Dendrimer-Mediated Transfer Printing of DNA and Rna Microarrays. J. Am. Chem. Soc. 2007, 129, 11593-11599.

7. Li, H. W.; Muir, B. V. O.; Fichet, G.; Huck, W. T. S. Nanocontact Printing: A Route to Sub-50-Nm-Scale Chemical and Biological Patterning. Langmuir 2003, 19, 1963-1965.

8. Escalante, M.; Zhao, Y. P.; Ludden, M. J. W.; Vermeij, R.; Olsen, J. D.; Berenschot, E.; Hunter, C. N.; Huskens, J.; Subramaniam, V.; Otto, C. Nanometer Arrays of Functional Light Harvesting Antenna 
Complexes by Nanoimprint Lithography and Host-Guest Interactions. J. Am. Chem. Soc. 2008, 130, 8892-8893.

9. Escalante, M.; Maury, P.; Bruinink, C. M.; van der Werf, K.; Olsen, J. D.; Timney, J. A.; Huskens, J.; Hunter, C. N.; Subramaniam, V.; Otto, C. Directed Assembly of Functional Light Harvesting Antenna Complexes onto Chemically Patterned Surfaces. Nanotechnology 2008, 19, -025101.

10. Rundqvist, J.; Hoh, J. H.; Haviland, D. B. Directed Immobilization of Protein-Coated Nanospheres to Nanometer-Scale Patterns Fabricated by Electron Beam Lithography of Poly(Ethylene Glycol) Self-Assembled Monolayers. Langmuir 2006, 22, 5100-5107.

11. Rundqvist, J.; Mendoza, B.; Werbin, J. L.; Heinz, W. F.; Lemmon, C.; Romer, L. H.; Haviland, D. B.; Hoh, J. H. High Fidelity Functional Patterns of an Extracellular Matrix Protein by Electron Beam-Based Inactivation. J. Am. Chem. Soc. 2007, 129, 59-67.

12. Glezos, N.; Misiakos, K.; Kakabakos, S.; Petrou, P.; Terzoudi, G. Electron Beam Patterning of Biomolecules. Biosens. Bioelectron. 2002, 17, 279-282.

13. Ngunjiri, J.; Garno, J. C. Afm-Based Lithography for Nanoscale Protein Assays. Anal. Chem. 2008, 80, 1361-1369.

14. Piner, R. D.; Zhu, J.; Xu, F.; Hong, S. H.; Mirkin, C. A. "Dip-Pen" Nanolithography. Science 1999, 283, 661-663.

15. Binnig, G.; Gerber, C.; Stoll, E.; Albrecht, T. R.; Quate, C. F. Atomic Resolution with Atomic Force Microscope. Europhys. Lett. 1987, 3, 1281-1286.

16. Jaschke, M.; Butt, H. J. Deposition of Organic Material by the Tip of a Scanning Force Microscope. Langmuir 1995, 11, 1061-1064.

17. Kramer, S.; Fuierer, R. R.; Gorman, C. B. Scanning Probe Lithography Using Self-Assembled Monolayers. Chem. Rev. 2003, 103, 4367-4418.

18. Thundat, T.; Zheng, X. Y.; Chen, G. Y.; Warmack, R. J. Role of Relative-Humidity in Atomic-Force Microscopy Imaging. Surf. Sci. 1993, 294, L939-L943.

19. Piner, R. D.; Mirkin, C. A. Effect of Water on Lateral Force Microscopy in Air. Langmuir 1997, 13, 6864-6868.

20. Haaheim, J.; Eby, R.; Nelson, M.; Fragala, J.; Rosner, B.; Zhang, H.; Athas, G. Dip Pen Nanolithography (Dpn): Process and Instrument Performance with Nanoink's Nscriptor System. Ultramicroscopy 2005, 103, 117-132.

21. Ginger, D. S.; Zhang, H.; Mirkin, C. A. The Evolution of Dip-Pen Nanolithography. Angew. Chem., Int. Ed. 2004, 43, 30-45.

22. Salaita, K.; Wang, Y. H.; Mirkin, C. A. Applications of Dip-Pen Nanolithography. Nat. Nanotechnol. 2007, 2, 145-155.

23. Jung, H.; Dalal, C. K.; Kuntz, S.; Shah, R.; Collier, C. P. Surfactant Activated Dip-Pen Nanolithography. Nano Lett. 2004, 4, 2171-2177.

24. Senesi, A. J.; Rozkiewicz, D. I.; Reinhoudt, D. N.; Mirkin, C. A. Agarose-Assisted Dip-Pen Nanolithography of Oligonucleotides and Proteins. Acs Nano 2009, 3, 2394-2402.

25. Giam, L. R.; Wang, Y. H.; Mirkin, C. A. Nanoscale Molecular Transport: The Case of Dip-Pen Nanolithography. J. Phys. Chem. A 2009, 113, 3779-3782.

26. Sheehan, P. E.; Whitman, L. J. Thiol Diffusion and the Role of Humidity in "Dip Pen Nanolithography". Phys. Rev. Lett. 2002, 88, -. 
27. Rozhok, S.; Piner, R.; Mirkin, C. A. Dip-Pen Nanolithography: What Controls Ink Transport? J. Phys. Chem. B 2003, 107, 751-757.

28. Schwartz, P. V. Molecular Transport from an Atomic Force Microscope Tip: A Comparative Study of Dip-Pen Nanolithography. Langmuir 2002, 18, 4041-4046.

29. Demers, L. M.; Ginger, D. S.; Park, S. J.; Li, Z.; Chung, S. W.; Mirkin, C. A. Direct Patterning of Modified Oligonucleotides on Metals and Insulators by Dip-Pen Nanolithography. Science 2002, 296, 1836-1838.

30. Lee, K. B.; Lim, J. H.; Mirkin, C. A. Protein Nanostructures Formed Via Direct-Write Dip-Pen Nanolithography. J. Am. Chem. Soc. 2003, 125, 5588-5589.

31. Weeks, B. L.; Vaughn, M. W.; DeYoreo, J. J. Direct Imaging of Meniscus Formation in Atomic Force Microscopy Using Environmental Scanning Electron Microscopy. Langmuir 2005, 21, 8096-8098.

32. Peterson, E. J.; Weeks, B. L.; De Yoreo, J. J.; Schwartz, P. V. Effect of Environmental Conditions on Dip Pen Nanolithography of Mercaptohexadecanoic Acid. J. Phys. Chem. B 2004, 108, 15206-15210.

33. Weeks, B. L.; DeYoreo, J. J. Dynamic Meniscus Growth at a Scanning Probe Tip in Contact with a Gold Substrate. J. Phys. Chem. B 2006, 110, 10231-10233.

34. Jang, J. Y.; Schatz, G. C.; Ratner, M. A. Liquid Meniscus Condensation in Dip-Pen Nanolithography. J. Chem. Phys. 2002, 116, 3875-3886.

35. http://www.nanoink.net/TheChemistry.htm, NanoInk.

36. Amro, N. A.; Xu, S.; Liu, G. Y. Patterning Surfaces Using Tip-Directed Displacement and Self-Assembly. Langmuir 2000, 16, 3006-3009.

37. Garno, J. C.; Yang, Y. Y.; Amro, N. A.; Cruchon-Dupeyrat, S.; Chen, S. W.; Liu, G. Y. Precise Positioning of Nanoparticles on Surfaces Using Scanning Probe Lithography. Nano Lett. 2003, 3, 389-395.

38. Li, Y.; Maynor, B. W.; Liu, J. Electrochemical Afm "Dip-Pen" Nanolithography. J. Am. Chem. Soc. 2001, 123, 2105-2106.

39. Cai, Y. G.; Ocko, B. M. Electro Pen Nanolithography. J. Am. Chem. Soc. 2005, 127, 16287-16291.

40. Wang, X. F.; Ryu, K. S.; Bullen, D. A.; Zou, J.; Zhang, H.; Mirkin, C. A.; Liu, C. Scanning Probe Contact Printing. Langmuir 2003, 19, 8951-8955.

41. Hong, S. H.; Mirkin, C. A. A Nanoplotter with Both Parallel and Serial Writing Capabilities. Science 2000, 288, 1808-1811.

42. Mirkin, C. A.; Hong, S. H.; Demers, L. Dip-Pen Nanolithography: Controlling Surface Architecture on the Sub-100 Nanometer Length Scale. Chemphyschem 2001, 2, 37-39.

43. Zhang, M.; Bullen, D.; Chung, S. W.; Hong, S.; Ryu, K. S.; Fan, Z. F.; Mirkin, C. A.; Liu, C. A Mems Nanoplotter with High-Density Parallel Dip-Pen Manolithography Probe Arrays. Nanotechnology 2002, 13, 212-217.

44. Liu, C. Parallel Scanning Probe Arrays: Their Applications. Mater. Today 2008, 11, 22-29.

45. Salaita, K.; Wang, Y. H.; Fragala, J.; Vega, R. A.; Liu, C.; Mirkin, C. A. Massively Parallel Dip-Pen Nanolithography with 55000-Pen Two-Dimensional Arrays. Angew. Chem., Int. Ed. 2006, 45, 7220-7223.

46. Haaheim, J.; Nafday, O. A. Dip Pen Nanolithography (R): A "Desktop Nanofab (Tm)" Approach Using High-Throughput Flexible Nanopatterning. Scanning 2008, 30, 137-150. 
47. Salaita, K.; Lee, S. W.; Wang, X. F.; Huang, L.; Dellinger, T. M.; Liu, C.; Mirkin, C. A. Sub-100 Nm, Centimeter-Scale, Parallel Dip-Pen Nanolithography. Small 2005, 1, 940-945.

48. Lenhert, S.; Sun, P.; Wang, Y. H.; Fuchs, H.; Mirkin, C. A. Massively Parallel Dip-Pen Nanolithography of Heterogeneous Supported Phospholipid Multilayer Patterns. Small 2007, 3, 71-75.

49. Zou, J.; Bullen, D.; Wang, X. F.; Liu, C.; Mirkin, C. A. Conductivity-Based Contact Sensing for Probe Arrays in Dip-Pen Nanolithography. Appl. Phys. Lett. 2003, 83, 581-583.

50. Bullen, D.; Chung, S. W.; Wang, X. F.; Zou, J.; Mirkin, C. A.; Liu, C. Parallel Dip-Pen Nanolithography with Arrays of Individually Addressable Cantilevers. Appl. Phys. Lett. 2004, 84, 789-791.

51. Wang, X. F.; Bullen, D. A.; Zou, J.; Liu, C.; Mirkin, C. A. Thermally Actuated Probe Array for Parallel Dip-Pen Nanolithography. J. Vac. Sci. Technol., B 2004, 22, 2563-2567.

52. Bullen, D.; Wang, X. F.; Zou, J.; Chung, S. W.; Mirkin, C. A.; Liu, C. Design, Fabrication, and Characterization of Thermally Actuated Probe Arrays for Dip Pen Nanolithography. J. Microelctromec. Sys. 2004, 13, 594-602.

53. Wang, X. F.; Liu, C. Multifunctional Probe Array for Nano Patterning and Imaging. Nano Lett. 2005, 5 , 1867-1872.

54. Bullen, D.; Liu, C. Electrostatically Actuated Dip Pen Nanolithography Probe Arrays. Sensors and Actuators a-Physical 2006, 125, 504-511.

55. Smith, J. C.; Lee, K. B.; Wang, Q.; Finn, M. G.; Johnson, J. E.; Mrksich, M.; Mirkin, C. A. Nanopatterning the Chemospecific Immobilization of Cowpea Mosaic Virus Capsid. Nano Letters 2003, 3, 883-886.

56. Lee, M.; Kang, D. K.; Yang, H. K.; Park, K. H.; Choe, S. Y.; Kang, C.; Chang, S. I.; Han, M. H.; Kang, I. C. Protein Nanoarray on Prolinker (Tm) Surface Constructed by Atomic Force Microscopy Dip-Pen Nanolithography for Analysis of Protein Interaction. Proteomics 2006, 6, 1094-1103.

57. Kwak, S. K.; Lee, G. S.; Ahn, D. J.; Choi, J. W. Pattern Formation of Cytochrome C by Microcontact Printing and Dip-Pen Nanolithography. Mater. Sci. Eng., C 2004, 24, 151-155.

58. Nam, J. M.; Han, S. W.; Lee, K. B.; Liu, X. G.; Ratner, M. A.; Mirkin, C. A. Bioactive Protein Nanoarrays on Nickel Oxide Surfaces Formed by Dip-Pen Nanolithography. Angew. Chem., Int. Ed. 2004, 43, 1246-1249.

59. Lim, J. H.; Ginger, D. S.; Lee, K. B.; Heo, J.; Nam, J. M.; Mirkin, C. A. Direct-Write Dip-Pen Nanolithography of Proteins on Modified Silicon Oxide Surfaces. Angew. Chem., Int. Ed. 2003, 42, 2309-2312.

60. Lee, K. B.; Park, S. J.; Mirkin, C. A.; Smith, J. C.; Mrksich, M. Protein Nanoarrays Generated by Dip-Pen Nanolithography. Science 2002, 295, 1702-1705.

61. Noy, A.; Miller, A. E.; Klare, J. E.; Weeks, B. L.; Woods, B. W.; DeYoreo, J. J. Fabrication of Luminescent Nanostructures and Polymer Nanowires Using Dip-Pen Nanolithography. Nano Lett. 2002, 2, 109-112.

62. Wilson, D. L.; Martin, R.; Hong, S.; Cronin-Golomb, M.; Mirkin, C. A.; Kaplan, D. L. Surface Organization and Nanopatterning of Collagen by Dip-Pen Nanolithography. PNAS 2001, 98, 13660-13664.

63. Wu, C. C.; Xu, H. P.; Otto, C.; Reinhoudt, D. N.; Lammertink, R. G. H.; Huskens, J.; Subramaniam, V.; Velders, A. H. Porous Multilayer-Coated Afm Tips for Dip-Pen Nanolithography of Proteins. J. Am. Chem. Soc. 2009, 131, 7526-7527. 
64. Choi, D. S.; Yun, S. H.; An, Y. C.; Lee, M. J.; Kang, D. G.; Chang, S. I.; Kim, H. K.; Kim, K. M.; Lim, J. H. Nanopatterning Proteins with a Stamp Tip for Dip-Pen Nanolithography. Biochip J. 2007, 1, 200-203.

65. Valiokas, R.; Vaitekonis, A.; Klenkar, G.; Trinkunas, G.; Liedberg, B. Selective Recruitment of Membrane Protein Complexes onto Gold Substrates Patterned by Dip-Pen Nanolithography. Langmuir 2006, 22, 3456-3460.

66. Lee, K. B.; Kim, E. Y.; Mirkin, C. A.; Wolinsky, S. M. The Use of Nanoarrays for Highly Sensitive and Selective Detection of Human Immunodeficiency Virus Type 1 in Plasma. Nano Lett. 2004, 4, 1869-1872.

67. Hyun, J.; Ahn, S. J.; Lee, W. K.; Chilkoti, A.; Zauscher, S. Molecular Recognition-Mediated Fabrication of Protein Nanostructures by Dip-Pen Lithography. Nano Lett. 2002, 2, 1203-1207.

68. Pena, D. J.; Raphael, M. P.; Byers, J. M. "Dip-Pen" Nanolithography in Registry with Photolithography for Biosensor Development. Langmuir 2003, 19, 9028-9032.

69. Zhang, H.; Lee, K. B.; Li, Z.; Mirkin, C. A. Biofunctionalized Nanoarrays of Inorganic Structures Prepared by Dip-Pen Nanolithography. Nanotechnology 2003, 14, 1113-1117.

70. Vega, R. A.; Maspoch, D.; Salaita, K.; Mirkin, C. A. Nanoarrays of Single Virus Particles. Angew. Chem., Int. Ed. 2005, 44, 6013-6015.

71. Lee, S. W.; Oh, B. K.; Sanedrin, R. G.; Salaita, K.; Fujigaya, T.; Mirkin, C. A. Biologically Active Protein Nanoarrays Generated Using Parallel Dip-Pen Nanolithography. Adv. Mater. 2006, 18, 1133-1136.

72. Vega, R. A.; Maspoch, D.; Shen, C. K. F.; Kakkassery, J. J.; Chen, B. J.; Lamb, R. A.; Mirkin, C. A. Functional Antibody Arrays through Metal Ion-Affinity Templates. Chembiochem 2006, 7, 1653-1657.

73. Kim, J. D.; Ahn, D. G.; Oh, J. W.; Park, W. J.; Jung, H. I. Ribosome Display and Dip-Pen Nanolithography for the Fabrication of Protein Nanoarrays. Adv. Mater. 2008, 20, 3349-3353.

74. Kim, K. H.; Kim, J. D.; Kim, Y. J.; Kong, S. H.; Jung, S. Y.; Jung, H. Protein Immobilization without Purification Via Dip-Pen Nanolithography. Small 2008, 4, 1089-1094.

75. Sekula, S.; Fuchs, J.; Weg-Remers, S.; Nagel, P.; Schuppler, S.; Fragala, J.; Theilacker, N.; Franueb, M.; Wingren, C.; Ellmark, P., et al. Multiplexed Lipid Dip-Pen Nanolithography on Subcellular Scales for the Templating of Functional Proteins and Cell Culture. Small 2008, 4, 1785-1793.

76. Cheung, C. L.; Camarero, J. A.; Woods, B. W.; Lin, T. W.; Johnson, J. E.; De Yoreo, J. J. Fabrication of Assembled Virus Nanostructures on Templates of Chemoselective Linkers Formed by Scanning Probe Nanolithography. J. Am. Chem. Soc. 2003, 125, 6848-6849.

77. Li, B.; Zhang, Y.; Hu, J.; Li, M. Q. Fabricating Protein Nanopatterns on a Single DNA Molecule with Dip-Pen Nanolithography. Ultramicroscopy 2005, 105, 312-315.

78. Wu, C. C.; Reinhoudt, D. N.; Otto, C.; Velders, A. H.; Subramaniam, V. Protein Immobilization on Ni(Ii) Ion Patterns Prepared by Microcontact Printing and Dip-Pen Nanolithography. Acs Nano 2010, in press.

79. Rakickas, T.; Gavutis, M.; Reichel, A.; Piehler, J.; Liedberg, B.; Valiokas, R. Protein-Protein Interactions in Reversibly Assembled Nanopatterns. Nano Lett. 2008, 8, 3369-3375.

80. Wei, J. H.; Kacar, T.; Tamerler, C.; Sarikaya, M.; Ginger, D. S. Nanopatterning Peptides as Bifunctional Inks for Templated Assembly. Small 2009, 5, 689-693.

81. Zhou, X. Z.; Chen, Y. H.; Li, B.; Lu, G.; Boey, F. Y. C.; Ma, J.; Zhang, H. Controlled Growth of Peptide Nanoarrays on Si/Siox Substrates. Small 2008, 4, 1324-1328. 
82. Sistiabudi, R.; Ivanisevic, A. Dip-Pen Nanolithography of Bioactive Peptides on Collagen-Terminated Retinal Membrane. Adv. Mater. 2008, 20, 3678-3681.

83. Hoover, D. K.; Chan, E. W. L.; Yousaf, M. N. Asymmetric Peptide Nanoarray Surfaces for Studies of Single Cell Polarization. J. Am. Chem. Soc. 2008, 130, 3280-3281.

84. Agarwal, G.; Sowards, L. A.; Naik, R. R.; Stone, M. O. Dip-Pen Nanolithography in Tapping Mode. $J$. Am. Chem. Soc. 2003, 125, 580-583.

85. Cho, Y.; Ivanisevic, A. Siox Surfaces with Lithographic Features Composed of a Tat Peptide. J. Phys. Chem. B 2004, 108, 15223-15228.

86. Jiang, H. Z.; Stupp, S. I. Dip-Pen Patterning and Surface Assembly of Peptide Amphiphiles. Langmuir 2005, 21, 5242-5246.

87. Hyun, J.; Lee, W. K.; Nath, N.; Chilkoti, A.; Zauscher, S. Capture and Release of Proteins on the Nanoscale by Stimuli-Responsive Elastin-Like Polypeptide "Switches". J. Am. Chem. Soc. 2004, 126, 7330-7335.

88. Cho, Y.; Ivanisevic, A. Tat Peptide Immobilization on Gold Surfaces: A Comparison Study with a Thiolated Peptide and Alkylthiols Using Afm, Xps, and Ft-Irras. J. Phys. Chem. B 2005, 109, 6225-6232.

89. Sistiabudi, R.; Ivanisevic, A. Patterning of Polypeptides on a Collagen-Terminated Tissue Surface. $J$. Phys. Chem. C 2007, 111, 11676-11681.

90. Nyamjav, D.; Ivanisevic, A. Alignment of Long DNA Molecules on Templates Generated Via Dip-Pen Nanolithography. Adv. Mater. 2003, 15, 1805-1809.

91. Demers, L. M.; Park, S. J.; Taton, T. A.; Li, Z.; Mirkin, C. A. Orthogonal Assembly of Nanoparticle Building Blocks on Dip-Pen Nanolithographically Generated Templates of DNA. Angew. Chem., Int. Ed. 2001, 40, 3071-3073.

92. Nyamjav, D.; Ivanisevic, A. Templates for DNA-Templated Fe3o4 Nanoparticles. Biomaterials 2005, 26, 2749-2757.

93. Jonkheijm, P.; Weinrich, D.; Schroder, H.; Niemeyer, C. M.; Waldmann, H. Chemical Strategies for Generating Protein Biochips. Angew. Chem., Int. Ed. 2008, 47, 9618-9647.

94. Rozkiewicz, D. I. Covalent Microcontact Printing of Biomolecules. University of Twente, Enschede, 2007.

95. You, C.; Bhagawati, M.; Brecht, A.; Piehler, J. Affinity Capturing for Targeting Proteins into Micro and Nanostructures. Anal. Bioanal. Chem. 2009, 393, 1563-1570.

96. Ariga, K.; Nakanishi, T.; Michinobu, T. Immobilization of Biomaterials to Nano-Assembled Films (Self-Assembled Monolayers, Langmuir-Blodgett Films, and Layer-by-Layer Assemblies) and Their Related Functions. J. Nanosci. Nanotechnol. 2006, 6, 2278-2301.

97. Jung, H.; Kulkarni, R.; Collier, C. P. Dip-Pen Nanolithography of Reactive Alkoxysilanes on Glass. J. Am. Chem. Soc. 2003, 125, 12096-12097.

98. Liu, M.; Amro, N. A.; Liu, G. Y. Nanografting for Surface Physical Chemistry. Annu. Rev. Phys. Chem. 2008, 59, 367-386.

99. Wadu-Mesthrige, K.; Amro, N. A.; Garno, J. C.; Xu, S.; Liu, G. Y. Fabrication of Nanometer-Sized Protein Patterns Using Atomic Force Microscopy and Selective Immobilization. Biophys. J. 2001, 80, 1891-1899.

100. Wadu-Mesthrige, K.; Xu, S.; Amro, N. A.; Liu, G. Y. Fabrication and Imaging of Nanometer-Sized Protein Patterns. Langmuir 1999, 15, 8580-8583. 
101. Kenseth, J. R.; Harnisch, J. A.; Jones, V. W.; Porter, M. D. Investigation of Approaches for the Fabrication of Protein Patterns by Scanning Probe Lithography. Langmuir 2001, 17, 4105-4112.

102. Cheung, C. L.; Chung, S. W.; Chatterji, A.; Lin, T. W.; Johnson, J. E.; Hok, S.; Perkins, J.; De Yoreo, J. J. Physical Controls on Directed Virus Assembly at Nanoscale Chemical Templates. J. Am. Chem. Soc. 2006, 128, 10801-10807.

103. Lee, M. V.; Nelson, K. A.; Hutchins, L.; Becerril, H. A.; Cosby, S. T.; Blood, J. C.; Wheeler, D. R.; Davis, R. C.; Woolley, A. T.; Harb, J. N., et al. Nanografting of Silanes on Silicon Dioxide with Applications to DNA Localization and Copper Electroless Deposition. Chem. Mater. 2007, 19, 5052-5054.

104. Liu, M. Z.; Amro, N. A.; Chow, C. S.; Liu, G. Y. Production of Nanostructures of DNA on Surfaces. Nano Lett. 2002, 2, 863-867.

105. Hu, Y.; Das, A.; Hecht, M. H.; Scoles, G. Nanografting De Novo Proteins onto Gold Surfaces. Langmuir 2005, 21, 9103-9109.

106. Case, M. A.; McLendon, G. L.; Hu, Y.; Vanderlick, T. K.; Scoles, G. Using Nanografting to Achieve Directed Assembly of De Novo Designed Metalloproteins on Gold. Nano Lett. 2003, 3, 425-429.

107. Tinazli, A.; Piehler, J.; Beuttler, M.; Guckenberger, R.; Tampe, R. Native Protein Nanolithography That Can Write, Read and Erase. Nat. Nanotechnol. 2007, 2, 220-225.

108. Takeda, S.; Nakamura, C.; Miyamoto, C.; Nakamura, N.; Kageshima, M.; Tokumoto, H.; Miyake, J. Lithographing of Biomolecules on a Substrate Surface Using an Enzyme-Immobilized Afm Tip. Nano Lett. 2003, 3, 1471-1474.

109. Nakamura, C.; Miyamoto, C.; Obataya, I.; Takeda, S.; Yabuta, M.; Miyake, J. Enzymatic Nanolithography of Fret Peptide Layer Using V8 Protease-Immobilized Afm Probe. Biosens. Bioelectron. 2007, 22, 2308-2314.

110. Hyun, J.; Kim, J.; Craig, S. L.; Chilkoti, A. Enzymatic Nanolithography of a Self-Assembled Oligonucleotide Monolayer on Gold. J. Am. Chem. Soc. 2004, 126, 4770-4771.

111. Tang, Q. L.; Zhang, Y. X.; Chen, L. H.; Yan, F. N.; Wang, R. Protein Delivery with Nanoscale Precision. Nanotechnology 2005, 16, 1062-1068.

112. Naujoks, N.; Stemmer, A. Using Local Surface Charges for the Fabrication of Protein Patterns. Colloids Surf., A 2004, 249, 69-72.

113. Sarveswaran, K.; Hu, W. C.; Huber, P. W.; Bernstein, G. H.; Lieberman, M. Deposition of DNA Rafts on Cationic Sams on Silicon [100]. Langmuir 2006, 22, 11279-11283.

114. Gu, J. H.; Yam, C. M.; Li, S.; Cai, C. Z. Nanometric Protein Arrays on Protein-Resistant Monolayers on Silicon Surfaces. J. Am. Chem. Soc. 2004, 126, 8098-8099.

115. Pavlovic, E.; Oscarsson, S.; Quist, A. P. Nanoscale Site-Specific Immobilization of Proteins through Electroactivated Disulfide Exchange. Nano Lett. 2003, 3, 779-781.

116. Agarwal, G.; Naik, R. R.; Stone, M. O. Immobilization of Histidine-Tagged Proteins on Nickel by Electrochemical Dip Pen Nanolithography. J. Am. Chem. Soc. 2003, 125, 7408-7412.

117. Qin, G. T.; Cai, C. Z. Sub-10-Nm Patterning of Oligo(Ethylene Glycol) Monolayers on Silicon Surfaces Via Local Oxidation Using a Conductive Atomic Force Microscope. Nanotechnology 2009, 20, -355306.

118. Taha, H.; Marks, R. S.; Gheber, L. A.; Rousso, I.; Newman, J.; Sukenik, C.; Lewis, A. Protein Printing with an Atomic Force Sensing Nanofountainpen. Appl. Phys. Lett. 2003, 83, 1041-1043. 
119. Xu, J. T.; Lynch, M.; Huff, J. L.; Mosher, C.; Vengasandra, S.; Ding, G. F.; Henderson, E. Microfabricated Quill-Type Surface Patterning Tools for the Creation of Biological Micro/Nano Arrays. Biomed. Microdevices 2004, 6, 117-123.

120. Moldovan, N.; Kim, K. H.; Espinosa, H. D. Design and Fabrication of a Novel Microfluidic Nanoprobe. J. Microelctromec. S. 2006, 15, 204-213.

121. Moldovan, N.; Kim, K. H.; Espinosa, H. D. A Multi-Ink Linear Array of Nanofountain Probes. $J$. Micromech. Microeng. 2006, 16, 1935-1942.

122. Kim, K. H.; Sanedrin, R. G.; Ho, A. M.; Lee, S. W.; Moldovan, N.; Mirkin, C. A.; Espinosa, H. D. Direct Delivery and Submicrometer Patterning of DNA by a Nanofountain Probe. Adv. Mater. 2008, 20, 330-334.

123. Loh, O.; Lam, R.; Chen, M.; Moldovan, N.; Huang, H. J.; Ho, D.; Espinosa, H. D. Nanofountain-Probe-Based High-Resolution Patterning and Single-Cell Injection of Functionalized Nanodiamonds. Small 2009, 5, 1667-1674.

124. Loh, O. Y.; Ho, A. M.; Rim, J. E.; Kohli, P.; Patankar, N. A.; Espinosa, H. D. Electric Field-Induced Direct Delivery of Proteins by a Nanofountain Probe. PNAS 2008, 105, 16438-16443. 


\title{
Chapter 3
}

\section{Fabrication and Visualization of Metal-ion Patterns on Glass by Dip-Pen Nanolithography*}

\begin{abstract}
Fluorescent monolayers sensitive to metal ions were used as dip-pen nanolithography (DPN) substrates for the fabrication of patterns of $\mathrm{Ca}$ (II) ions. The driving force for the transfer of these ions from an atomic force microscope (AFM) tip to the surface is their complexation to the organic ligands on the monolayer. Using the fluorescent surfaces, the patterns could be visualized using a fluorescence microscope. A custom-built atomic force fluorescence microscope (AFFM), a combination of an atomic force microscope and a confocal fluorescence microscope, was used to deposit the metal ions onto the sensitive monolayers by DPN and to subsequently visualize the modulations of fluorescence intensity in a sequential write-read mode.
\end{abstract}

\footnotetext{
*Part of this chapter has been published in Basabe-Desmonts, L. ; Wu, C. C.\#; van der Werf, K. O.; Peter, M.; Bennink, M.; Otto, C.; Velders, A. H.; Reinhoudt, D. N.; Subramaniam, V.; Crego-Calama, M. ChemPhysChem 2008, 9, 1680-1687. (" shared first author)
} 


\subsection{Introduction}

Chemical sensing of ions and small organic molecules is significant for various fields including environmental science, medical diagnostics, and the food industry. ${ }^{1}$ High sensitivity and easy operation are two essential requirements for such sensors; fluorescence based approaches are among the most suitable candidates for chemical sensing because of the sensitivity and specificity of fluorescence, and the relative simplicity of the assays. ${ }^{1}$ The traditional design of a fluorescent indicator comprises a receptor and a fluorophore, which are responsible for recognizing the analyte and signaling the recognition event, respectively. These fluorescent indicators have to be incorporated into a solid support to function as a chemosensor. The most common approaches of incorporation reported to date are physical entrapment or covalent attachment of sensitive probes to a polymeric matrix, and confining the fluorophore indicators in space. Among these methods, surface-confinement of the chemical sensing system provides some advantages, e.g. avoiding problems of leaking, fast response times, easy and reproducible synthesis, and the introduction of additional chelating effects from the pre-organized surface platform. ${ }^{1,2}$

The sensitive monolayers previously developed in our group offer a simple and efficient method for detecting metal ions by observing the modulation of fluorescence intensity. ${ }^{3-5}$ This methodology is comprised of randomly functionalizing monolayers with different fluorophores and ligand molecules onto amino-terminated monolayers on glass substrates, as shown in Scheme 3.1. By taking advantage of the interaction of organic ligands with metal ions, these monolayer-functionalized glass slides become metal-ion sensing platforms. Modulation of the fluorescence properties, i.e. different degrees of fluorescence enhancement or quenching, occurs upon complexation of different kinds of metal ions by the organic monolayers on top of the glass slide. It has also been demonstrated previously that the metal-ion patterns at micrometer $(\mu \mathrm{m})$ scale can be fabricated on the sensitive monolayer-functionalized glass slides with microcontact printing $(\mu \mathrm{CP})^{2,4}$ and dip-pen nanolithography $(\mathrm{DPN})^{2}$ techniques, where the sensing properties of the fluorescent substrates allow the visualization of the patterns by confocal fluorescence microscopy. It is well-known that patterns with sub- $\mu \mathrm{m}$ feature sizes can be achieved easily with DPN. ${ }^{6-8}$ However, due to the intrinsic difficulty of finding the written pattern after transferring the sample from an atomic force microscope (AFM) to a confocal fluorescence microscope, only patterns with larger feature sizes (20 $\mu \mathrm{m}$ or larger) have been reported. ${ }^{2}$ 


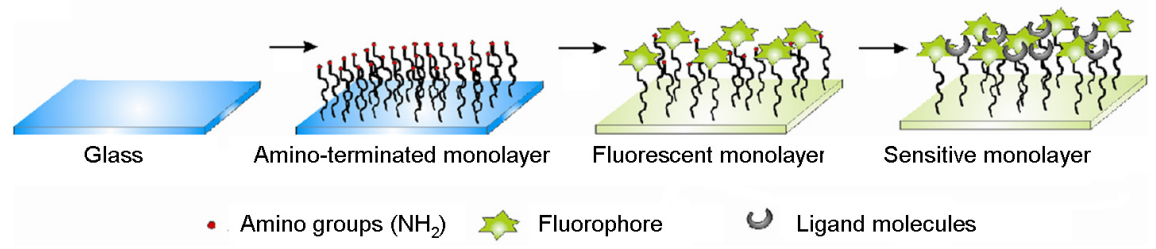

Scheme 3.1. General schematic of the fabrication procedure of the metal-ion-sensitive monolayer-functionalized glass substrates.

In this chapter, a modification of the protocol for preparing sensitive monolayers is described. The metal ion sensing property of the modified monolayers was confirmed by $\mu \mathrm{CP} . \mu \mathrm{CP}$ is a soft lithographic approach using an elastomeric stamp with relief features prepared from a solid master to transfer ink molecules onto the target hard surface, as shown in Scheme 3.2. Poly(dimethylsiloxane) (PDMS) is typically used to prepare soft stamps. The generation of patterns relies on the conformal contact between the stamp and the substrate. ${ }^{9}$ Subsequently, the controlled delivery of metal ions in well-defined $\mu \mathrm{m}$ and sub- $\mu \mathrm{m}$ patterns on discrete areas of the monolayer-functionalized glass by DPN with a custom-built instrument, the atomic force fluorescence microscope (AFFM), is presented. AFFM enables us to write metal ions and subsequent visualize in situ the written results in a sequential write-read mode.

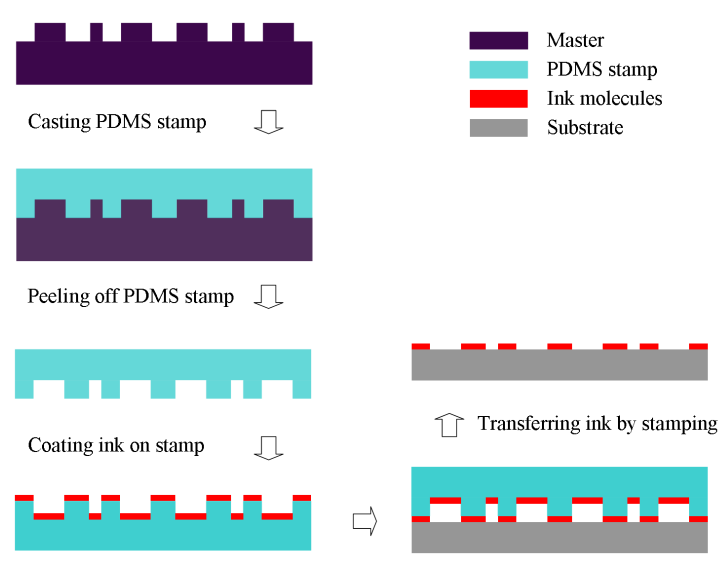

Scheme 3.2. Schematic representation of preparing PDMS stamp and the microcontact printing $(\mu C P)$ technique. 


\subsection{Results and Discussion}

\subsubsection{The Atomic force fluorescence microscope (AFFM)}

Scheme 3.3a shows the layout of the AFFM, which is an analysis tool combining an AFM and a confocal fluorescence microscope. A detailed description of AFFM has been reported by Kassies et al. ${ }^{10,11}$ AFFM enables the possibility to do DPN experiments and to immediately observe patterning results in situ, thereby facilitating the scaling down of feature sizes of the metal-ion patterns to sub- $\mu \mathrm{m}$ range without facing the difficulty of finding the written patterns back after moving samples from an AFM to a confocal fluorescence microscope. Scheme 3.3b depicts how the writing and visualization can be performed in situ. An AFM tip has to be brought into contact with a substrate right above the focus point of the objective lens while writing.

a)

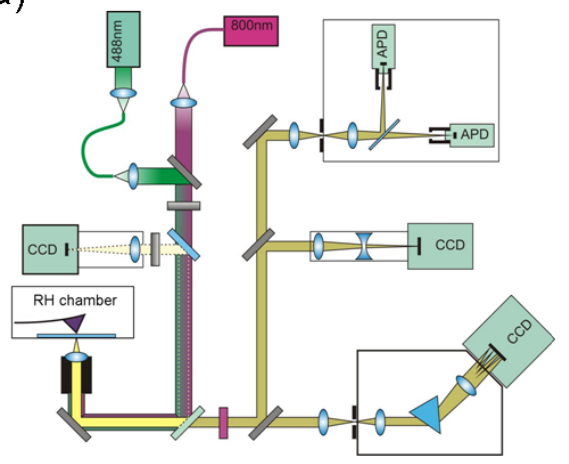

b)

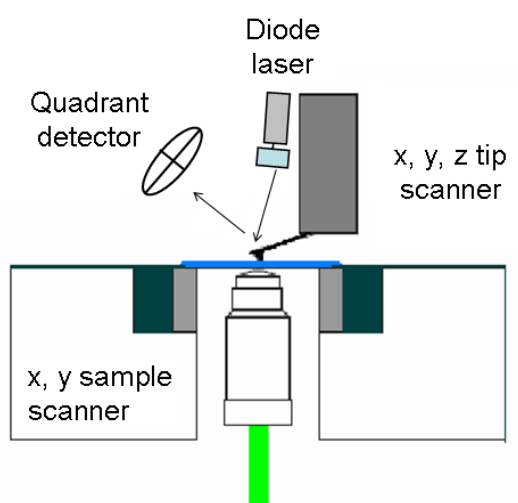

Scheme 3.3. (a) Schematic layout of the atomic force fluorescence microscope (AFFM) shows two excitation wavelengths of lasers, $488 \mathrm{~nm}$ and $800 \mathrm{~nm}$ and two different detection paths for photon accounting (APD) available. Spectral discrimination can be obtained by a prism-based spectrograph. (b) Schematic of DPN and in situ fluorescence visualization by AFFM.

Over the past years, several groups have presented their DPN experimental results mostly based on the studies of depositing mercaptohexadecanoic acid (MHA) or 1-octadecanethiol (ODT) on gold substrates and concluded that relative humidity (RH) is one of the important factors affecting the transportation of ink molecules from the tip 
surface to the substrate. ${ }^{12-17}$ In the original AFFM setup, there was no equipment for controlling relative humidity while writing ink molecules. Therefore, building a chamber with a relative humidity generator on the AFFM set-up has been necessary in order to have better control of DPN experiments.

The specific manner in which to build a humidity chamber on an AFM strongly depends on the original design of the instrument. Several requirements have to be considered including sealing, small volume, and avoiding exposing electronic components and optics to high humidity and temperature. Some approaches to building custom humidity chambers on an AFM have been reported. For example, Maxwell et al. used a small Perspex chamber to enclose the whole AFM scanner head and sample stage, or the lower portion of the AFM scanner tube. ${ }^{18}$ This approach is very straightforward but it takes a long time to reach a desired RH value due to the large volume of the chamber. Stukalov et al. presented another method to control RH near an AFM probe by only enclosing the sample and the cantilever holder. ${ }^{19}$ This kind of chamber is more suitable to adapt on the AFFM because the volume is small and enables one to quickly reach a desired RH value. Besides, not too many modifications to the AFFM have to be made. Photographs of the designed RH chamber and AFM head are shown in Figure 3.1a and $\mathrm{b}$. The diameter and height of the chamber are $\sim 5 \mathrm{~cm}$ and $\sim 1 \mathrm{~cm}$, respectively. A commercial sensor (SHT75, Sensirion, Switzerland) was placed into the chamber for monitoring relative humidity $(\mathrm{RH})$ and temperature while performing DPN experiments. It can be seen in Figure 3.1b that the chamber is not fully sealed because of the open access for the AFM nose and therefore there is a leakage of the flowing humid air. Nevertheless, the RH still can reach a desired value with $\pm 3 \%$ deviation within 20 minutes in the chamber. Moist air with different RH near the AFM tip was created by mixing dry and wet nitrogen $\left(\mathrm{N}_{2}\right)$ with varying ratios. (Figure $3.1 \mathrm{c}$ ) The wet $\mathrm{N}_{2}$ was generated by flowing dry $\mathrm{N}_{2}$ through two bottles of milliQ water followed by a bottle containing $5 \mathrm{~mm}$ diameter glass beads. ${ }^{18}$ This home-built $\mathrm{RH}$ chamber combined with the simple humidity generator is not suitable for performing DPN experiments under the condition of high RH because the electronic components of AFM exposed to moist air may be damaged. This humidity control system was only used to raise the RH to approximately $50 \%$ when the environmental humidity was too low. 


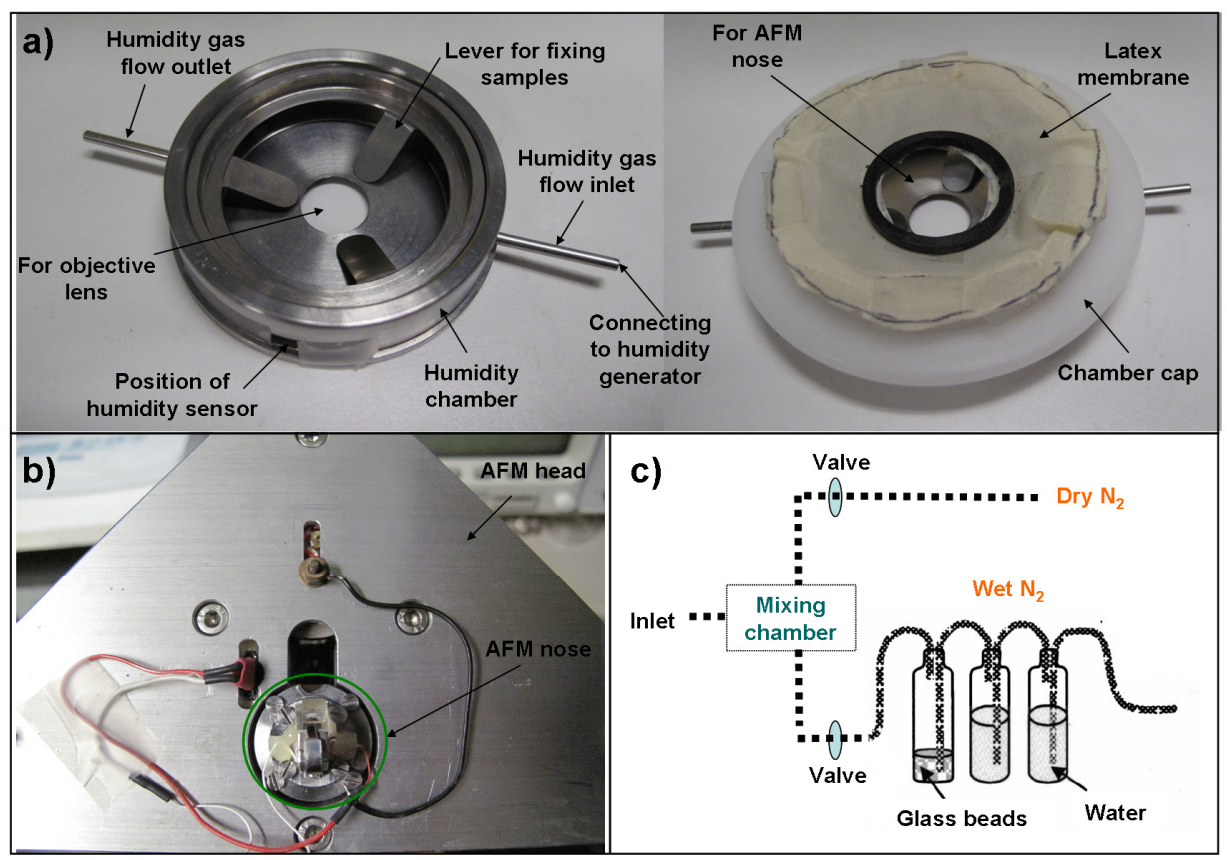

Figure 3.1. (a) Photographs of the home-built relative humidity chamber with (top left) and without (top right) the chamber cap. (b) Photographs of the AFM head. The AFM nose can access the substrate via the open area of chamber cap shown in (a). (c) Schematic illustration of the generator of moist air.

\subsubsection{Sensitive monolayers}

The monolayer functionalized-substrate chosen for DPN experiments was selected from a library of sensitive monolayers, presented by Basabe-Desmonts et al. previously, based on its high sensitivity for calcium ions. ${ }^{20}$ The synthesis of the sensitive monolayer is depicted in Scheme 3.4. A three-step procedure was followed. An amino-terminated monolayer was first formed by silylation of the glass substrates with (N-[3-(trimethoxysilyl)propyl]-ethylenediamine) (TPEDA). Subsequently the TPEDA monolayer was converted into a fluorescent monolayer by reacting amino groups with the fluorophore 5-(and-6)-carboxytetramethylrhodamine succinimidyl ester (TAMRA). The sensitive monolayer was obtained in the end by covalently attaching the ligand molecules, hexanoyl chloride, to the remaining amino groups. 


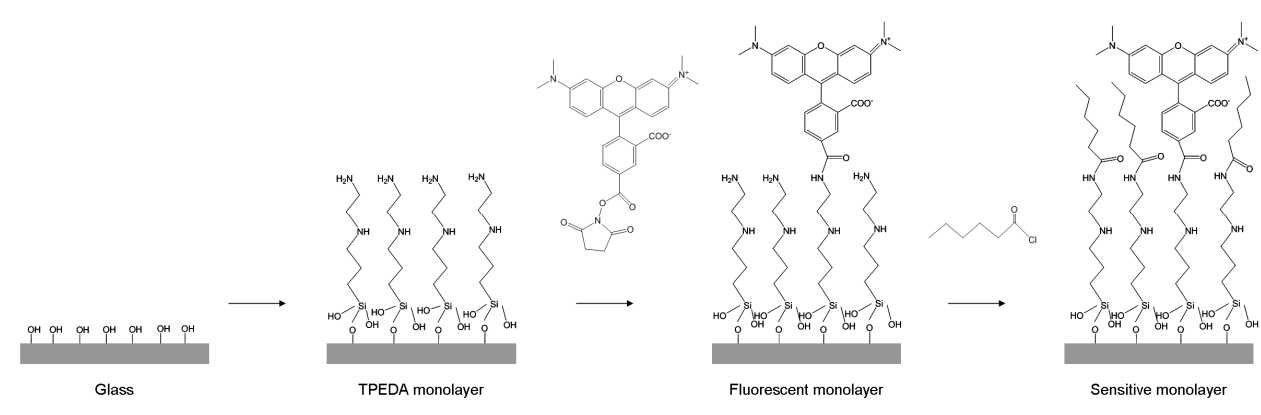

Scheme 3.4. Illustration of the preparation procedure of a sensitive monolayer on glass.

When beginning with DPN experiments, an experimental problem with the fluorescent monolayers prepared by following the original protocol came to light. It was found that the emission of fluorescence could be enhanced simply by scanning with 488 $\mathrm{nm}$ Argon laser light. We conjectured that the anomalous fluorescence enhancement might result from the dequenching of closely-packed fluorophore molecules on the top of the fluorescent monolayer. By scanning with the laser light, some self-quenchers were likely removed by photobleaching and consequently resulted in fluorescence enhancement.

To avoid any influence on the analyses of DPN results, modifying the original protocol for preparing fluorescent monolayers was necessary. The fluorescent monolayers prepared with different concentrations of TAMRA were examined by scanning laser light for two minutes. The corresponding fluorescence images are displayed in Figure 3.2. The effect of initial enhancement of fluorescence intensity was not visible only in the monolayers prepared in TAMRA solution of $0.01 \mathrm{mM}$.

Further, the fluorescent monolayers prepared with $0.07 \mathrm{mM}$ and $0.01 \mathrm{mM}$ TAMRA solutions were microcontact printed with metal ions to inspect if they still retained the ability to sense metal ions. The freshly prepared PDMS stamps were oxidized with UV/ozone cleaner to increase the wettability and then inked with $0.1 \mathrm{M}$ ethanol solutions of the perchlorate salts of $\mathrm{Ca}(\mathrm{II})$ ions. After $\mu \mathrm{CP}$ process, the fluorescent monolayers were imaged with a fluorescence microscope. Figure $3.3 \mathrm{a}$ and $3.3 \mathrm{~b}$ show the fluorescence images of fluorescent monolayers-functionalized substrates after printing with $\mathrm{Ca}(\mathrm{II})$ ions. The enhancement of fluorescence by the printed $\mathrm{Ca}(\mathrm{II})$ ions corresponds to the result 
published before. ${ }^{4,20}$ The $\mu \mathrm{CP}$ results show that the modified monolayers are capable of sensing metal ions and there is no conspicuous difference between the monolayer prepared from different concentrations of used fluorophore solutions. In accordance with these test experiments, fluorescent monolayers prepared at $0.01 \mathrm{mM}$ were chosen for DPN experiments.
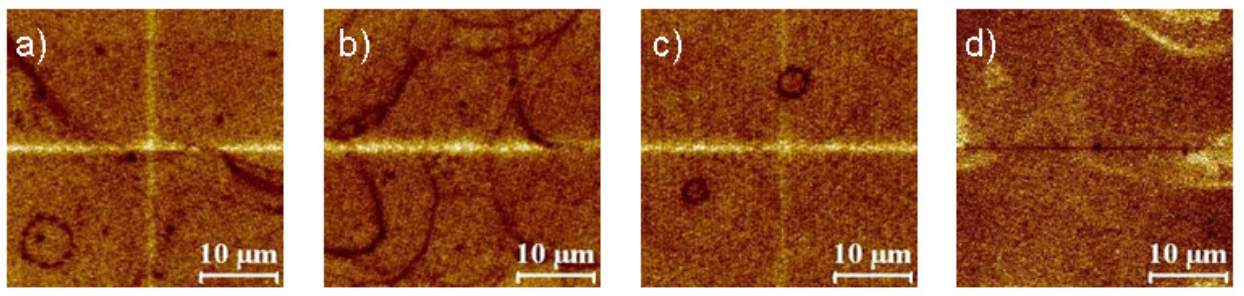

Figure 3.2. Fluorescence images of fluorescent monolayers prepared in (a) $0.07 \mathrm{mM}$, (b) $0.05 \mathrm{mM}$, (c) $0.03 \mathrm{mM}$, (d) $0.01 \mathrm{mM}$ TAMRA solutions were obtained by AFFM after continuous illumination with $488 \mathrm{~nm}$ laser light for two minutes during an intro-scanning routine.
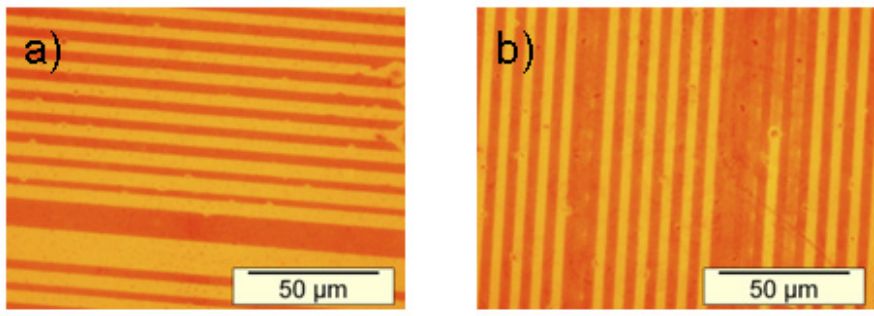

Figure 3.3. Fluorescence images of fluorescent monolayers prepared from (a) $0.07 \mathrm{mM}$ and (b) $0.01 \mathrm{mM}$ TAMRA solution acquired after microcontact printing with calcium metal ions. 


\subsubsection{Fabrication and visualization of metal-ion patterns}

\subsubsection{Writing metal ions at micron scale}

Commercial $\mathrm{Si}_{3} \mathrm{~N}_{4}$ AFM tips incubated in $0.1 \mathrm{M}$ ethanol solutions of the perchlorate salts of $\mathrm{Ca}(\mathrm{II})$ ions were used to generate metal-ion patterns on the sensitive monolayer-functionalized substrate. Sequential AFFM write-read experiments confirm an increase in the fluorescence upon complexation of $\mathrm{Ca}$ (II) ions to the sensitive monolayers. Figure 3.4 shows a $15 \times 15 \mu \mathrm{m}^{2}$ square written with $\mathrm{Ca}$ (II) ions. The bright square in the fluorescence image indicates the transfer of the metal ions onto the written area. The vertical edges and the central part of the square are brighter, suggesting that larger amounts of metal ions were transferred from the tip onto the sensitive monolayer at these specific locations. This is in agreement with the fact that the tip is in contact with the substrate for longer time in these areas. The center part corresponds to the intro-scanned process, which was performed before writing the whole square pattern in order to adjust the electronic offset signal to the tilt of the substrate. During this adjustment the $\mathrm{Ca}$ (II) ions-loaded tip scans the same line several times. The tip was also longer in contact with the substrate at the vertical edges of the square due to the change of direction between trace and retrace at the end of each scan line. The darker cross lines shown in Figure 3.4 resulted from the photobleaching of the substrate when scanning with the $488 \mathrm{~nm}$ Argon laser light before performing DPN.

a)

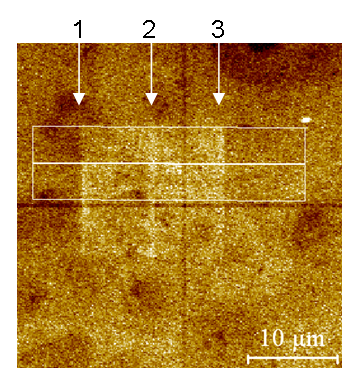

b)

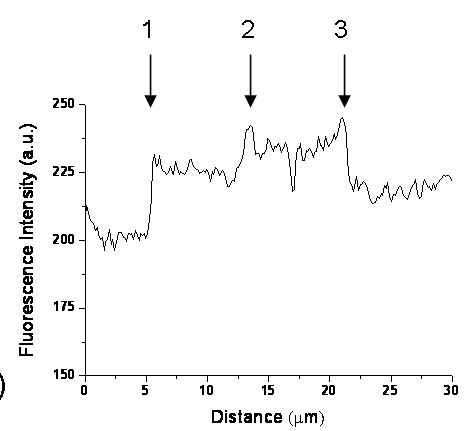

Figure 3.4. (a) Fluorescence image of a sensitive monolayer-funstionalized substrate obtained by AFFM. A square pattern $\left(15 x \quad 15 \quad \mu^{2}\right)$ was generated by dip-pen nanolithography using an AFM tip inked with Ca (II) ions. (b) Average fluorescence intensity profile of the area defined by the white lines. The arrows indicate the lines with higher fluorescence intensity. 


\subsubsection{Writing metal ions at sub-micron scale}

The fabrication and visualization of sub- $\mu$ scale metal-ion patterns have also been explored. Single lines of $\mathrm{Ca}$ (II) ions were deposited onto the substrates, and therefore bright lines with sub-micrometer widths were expected in the fluorescence images. During the experiments, we varied the scan speed between $\sim 18$ and $\sim 1.8 \mu \mathrm{m} / \mathrm{s}$ to observe the relation between the tip-substrate contact time and the level of modulation of fluorescence. It was expected that a slower speed (longer contact time between the tip and the substrate on a certain area) would result in a higher amount of ink molecules transferred to the substrate and therefore it would induce higher enhancement of fluorescence. Figures 3.5a and $3.5 \mathrm{c}$ show that the widths of lines written on a sensitive monolayer-functionalized substrate are $\sim 0.8 \mu \mathrm{m}$ and $\sim 0.6 \mu \mathrm{m}$ respectively. In Figure $3.5 \mathrm{a}$, six horizontal lines of $18 \mu \mathrm{m}$ in length were generated with six different scan speeds without retracting the tip during the whole DPN process. Each line was scanned 15 times and the fluorescence image was recorded immediately after completion of DPN of all six lines. As expected, slight differences in the brightness of the lines can be observed in Figure 3.5a. Lines written at a lower scan rate show higher fluorescence intensity, which is in agreement with a larger amount of transferred $\mathrm{Ca}$ (II) ions. Close inspection of the fluorescence intensity profile shown in Figure 3.5b exhibits a gradual increase of signal with the lower scan rates. However, the trend is reversed in the last two lines. This effect may be attributed to depletion of ink from the tip. Figure 3.5c shows two lines written with a scan rate of $\sim 1.8 \mu \mathrm{m} / \mathrm{s}$ for 5 and 10 minutes. In spite of the fact that one line was written for a longer time, the amplitude of the fluorescence emission from both lines was similar. We postulate that after scanning for a few minutes, the surface was saturated with $\mathrm{Ca}$ (II) ions leading to the observation of the maximum possible emission intensity. Some variations of line width between different experiments, such as those presented in Figure 3.5, have been observed. These differences might result from a different $\mathrm{RH}$ in the environment of the cantilever, or manufacturing differences of the tip. These effects have been already reported in literature. ${ }^{7,21,22}$ 
a)

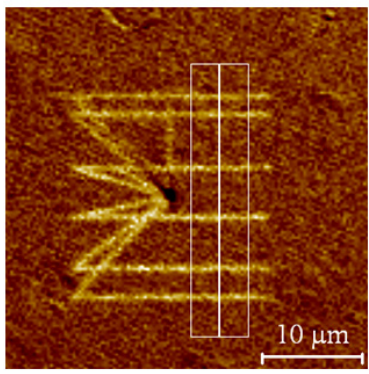

c)

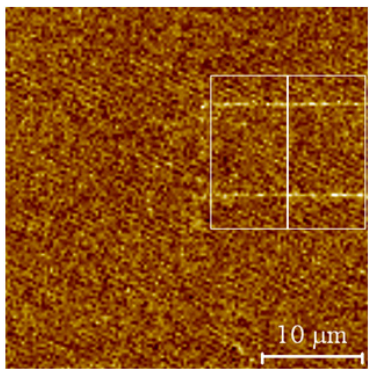

b)

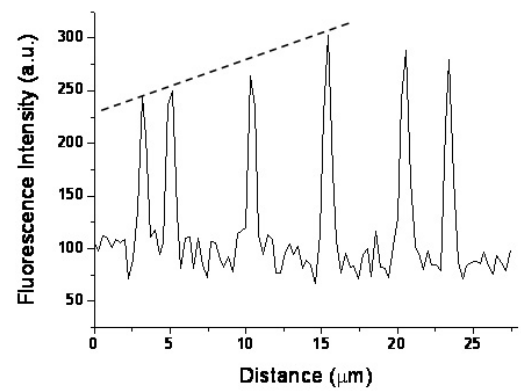

d)

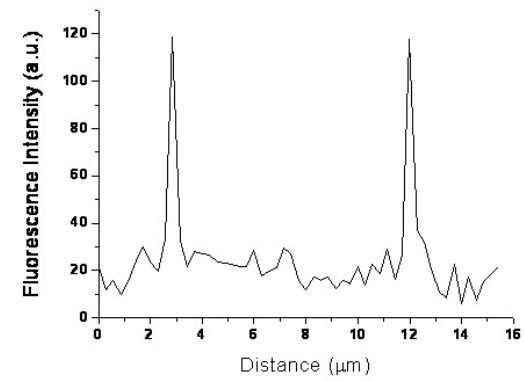

Figure 3.5. (a) Fluorescence image of a sensitive monolayer-functionalized substrate acquired immediately after DPN with the AFFM. Six horizontal lines (18 $\mu \mathrm{m}$ in length) were successively generated by using an AFM tip inked with Ca(II) ions using scanning speeds of 17.7, 14.1, 10.6, 7.1, 3.5 and $1.8 \mu \mathrm{m} / \mathrm{s}$, from top to bottom. (b) The average fluorescence intensity profile of the area inside the rectangle indicated in (a) The peaks from left to right correspond to the written lines from top to bottom. The dashed line indicates the increasing enhancement of fluorescence trend corresponding to a lower scanning speed (c) Fluorescent image of a sensitive monolayer-functionalized substrate acquired with the AFFM. Two horizontal lines (18 $\mu \mathrm{m}$ long) were generated by DPN using an AFM tip inked with Ca(II) ions for 5 (top) and 10 (bottom) minutes, respectively. The upper line was written first. (d) Average fluorescence intensity profile of the area defined by the white lines. The left peak corresponds to the upper written line. In both images a) and c) the background, which is the fluorescence image before DPN, was subtracted. 


\subsection{Conclusions}

In summary, the AFFM instrument used in this thesis work has been briefly described. A custom-built humidity chamber was designed for the AFFM for performing DPN experiments while controlling the relative humidity. Then it has been demonstrated that it is possible to fabricate $\mathrm{Ca}$ (II)-ion patterns, at micrometer and sub-micrometer scale, on the modified non-conductive sensitive monolayer-functionalized glass substrates with DPN. The driving force for the transfer of ink from the AFM tip to the substrate is the formation of $\mathrm{Ca}$ (II) ion complexes on the organic monolayer on the glass substrates. Such transfer of metal ions was revealed by an enhancement of the fluorescence response. The sensitive monolayer coated glass substrates used for the deposition offer a number of advantages, allowing optical visualization of the pattern even in cases in which the concentration of metal ions on the surface is so low that the AFM images do not exhibit any feature. Moreover, the combination of the topographic resolution of the AFM and the confocal fluorescence microscope in the AFFM enhances the possibilities of this approach by allowing immediate fluorescence imaging of the fabricated patterns in a sequential write-read mode in situ. Additionally, the fabrication of metal ion patterns by DPN can be used for the production of protein nanopatterns, which is described in detail in Chapter 4.

\subsection{Experimental}

\section{General procedures.}

All glassware used to prepare the monolayers was cleaned by sonicating for 60 minutes in a $2 \% \mathrm{v} / \mathrm{v}$ Hellmanex II solution in high purity water (MilliQ, 18.2 M $\Omega \mathrm{cm}$ ), and then rinsed thoroughly with MilliQ water, and dried in an ambient environment. Microscope glass slide substrates and $\mathrm{Si}_{3} \mathrm{~N}_{4}$ AFM cantilevers (NP, Veeco) were cleaned in piranha solution for 20 minutes and 1 minute, respectively, (concentrated $\mathrm{H}_{2} \mathrm{SO}_{4}$ and $33 \%$ aqueous $\mathrm{H}_{2} \mathrm{O}_{2}$ in a $3: 1$ ratio). [Warning: Piranha solution should be handled with caution: it has been reported to detonate unexpectedly.] The glass slides and cantilevers were then rinsed several times with Milli-Q water, and dried gently under a nitrogen stream before forming the monolayer.

\section{Monolayer preparation.}

Preparation of the amino terminated monolayer (TPEDA). Formation of the $\mathrm{N}$-[3-(trimethoxysilyl)propyl]-ethylenediamine monolayer (TPEDA) was achieved in a glovebox under an atmosphere of dry nitrogen. The freshly cleaned substrates were immersed in a $5 \mathrm{mM}$ solution of $N$-[3-(trimethoxysilyl)propyl]-ethylenediamine in freshly distilled toluene for 3.5 hours. 
After the substrates were taken from the solution, they were rinsed twice with toluene (under nitrogen atmosphere) to remove excess silane and avoid polymerization. The substrates were then removed from the glovebox and rinsed with ethanol and dichloromethane to remove physisorbed material. The following protocol was repeated twice: shaking of the slide in a beaker filled with $\mathrm{EtOH}$, then rinsing with a stream of $\mathrm{EtOH}$, followed by stirring in $\mathrm{CH}_{2} \mathrm{Cl}_{2}$, then rinsing with a stream of $\mathrm{CH}_{2} \mathrm{Cl}_{2}$. The slides were then dried under an air stream.

Preparation of sensitive monolayer. For the synthesis of the sensitive monolayers, the fluorescent monolayers have to be prepared first by attaching the fluorophores to the TPEDA monolayers. This step was achieved by immersing the TPEDA monolayers-functionalized slides into the acetonitrile solutions of different concentrations of the fluorophore, (5-(and-6)-carboxytetramethylrhodamine, succinimidyl ester (5(6)-TAMRA, SE) *mixed isomers)) (TAMRA) for 4 hours. Then the substrates were taken out from the solution and rinsed with $\mathrm{CH}_{3} \mathrm{CN}$, EtOH, and $\mathrm{CH}_{2} \mathrm{Cl}_{2}$ to remove physisorbed material. The slides were then dried under an air stream. The fluorescent monolayers-functionalized slides were subsequently immersed in the $50 \mathrm{mM}$ acetonitrile solution of hexanoyl chloride with $100 \mu \mathrm{LEt}_{3} \mathrm{~N}$ to yield sensitive monolayers. The reaction was carried out under an atmosphere of dry $\mathrm{N}_{2}$ for 16 hours. After the substrates were taken out from the solution, they were rinsed sequentially with $\mathrm{CH}_{3} \mathrm{CN}$, EtOH, and $\mathrm{CH}_{2} \mathrm{Cl}_{2}$ to remove physisorbed material. The slides were then dried under an air stream. All monolayer-functionalized glass slides were then sonicated for 1 minute in a beaker filled with $\mathrm{CH}_{2} \mathrm{Cl}_{2}$, then rinsed again with $\mathrm{CH}_{2} \mathrm{Cl}_{2}$ and dried under an air stream, to assure clean sensitive monolayers. The formation of monolayers on the glass slides was characterized by ellipsometry, water contact angle goniometry, fluorescent spectroscopy and XPS as reported elsewhere. $^{20}$

\section{Microcontact printing ( $\mu \mathrm{CP})$ with $\mathrm{Ca}(\mathrm{II})$ ions on sensitive monolayers.}

A 10:1 (v/v) mixture of poly(dimethylsiloxane) (PDMS) and curing agent (Sylgard 184, Dow Corning) was cast against patterned silicon master and cured at $60{ }^{\circ} \mathrm{C}$ overnight. The PDMS stamps were obtained after carefully peeling them off from the silicon master after curing. Prior to inking process, all the freshly prepared PDMS stamps were oxidized by exposure to UV/ozone cleaner for 30 minutes to increase the wettability of stamps' surfaces. The oxidized stamps were immediately incubated into the $10^{-1} \mathrm{M}$ ethanol solutions of the perchlorate salts of $\mathrm{Ca}(\mathrm{II})$ ions for at least 20 minutes. After inking, the stamps were dried under a stream of $\mathrm{N}_{2}$ to blow the excess ink away. The inked stamps were brought into conformal contact with sensitive monolayers-functionalized substrates for 3 minutes without the use of external pressure. The sensitive monolayers-functionalized substrates printed with $\mathrm{Ca}$ (II) ions were examined with a fluorescence microscope. 


\section{Dip-pen nanolithography (DPN) with Ca(II) ions on sensitive monolayers.}

Commercial $\mathrm{Si}_{3} \mathrm{~N}_{4}$ AFM cantilevers with nominal spring constant of $0.05 \mathrm{~N} / \mathrm{m}$ were used. Immediately before a DPN experiment, cantilevers were rinsed rigorously with ethanol and dried under a $\mathrm{N}_{2}$ stream very gently. After cleaning, the cantilevers were exposed to UV light for 30 minutes to break down contaminants and make the cantilever surface more hydrophilic. Cleaned cantilevers were then immersed into $0.1 \mathrm{M}$ ethanol solutions of the perchlorate salts of $\mathrm{Ca}$ (II) ions for 5 minutes and then dried in air. The $\mathrm{Ca}$ (II)-coated cantilever was immediately mounted on the AFM head to carry out DPN experiments on sensitive monolayers in contact mode with or without humid control at a temperature between 20 and $23{ }^{\circ} \mathrm{C}$. The trace of the scanned cantilever was consistent with the re-trace.

The $15 \times 15 \mu \mathrm{m}^{2}$ square-shaped patterns were written twice with a scan rate of $28 \mu \mathrm{m} / \mathrm{s}$. Furthermore, $18 \mu \mathrm{m}$ long fluorescent lines were fabricated with defined number of scans (15 scans) or controlled scan rate $(1.8 \mu \mathrm{m} / \mathrm{s})$. During the writing period, the fluorescence excitation light was switched off to prevent the samples from photobleaching. Immediately after DPN, the patterned substrate was excited with $488 \mathrm{~nm}$ Argon laser light and the emission of the fluorescence was recorded by an avalanche photodiode (APD) (SPCM-AQR-14, Perkin Elmer Optoelectronics). The acquired fluorescent images were analyzed by the commercial software SPIP (Image Metrology, Version 4.4.3.0).

\subsection{Acknowledgements}

Dr. Lourdes Basabe-Desmonts is acknowledged for the original idea of this project and her valuable comments, and Kees van der Werf is acknowledged for his kind help of designing the relative humidity chamber.

\subsection{References}

1. Basabe-Desmonts, L.; Reinhoudt, D. N.; Crego-Calama, M. Design of Fluorescent Materials for Chemical Sensing. Chem. Soc. Rev. 2007, 36, 993-1017.

2. Lourdes, B.-D. Fluorescent Self-Assembled Monolayers as New Sensing Materials. University of Twente, 2005.

3. Zimmerman, R.; Basabe-Desmonts, L.; van der Baan, F.; Reinhoudt, D. N.; Crego-Calama, M. A Combinatorial Approach to Surface-Confined Cation Sensors in Water. J. Mater. Chem. 2005, 15, 2772-2777.

4. Basabe-Desmonts, L.; Reinhoudt, D. N.; Crego-Calama, M. Combinatorial Fabrication of Fluorescent Patterns with Metal Ions Using Soft Lithography. Adv. Mater. 2006, 18, 1028-1032.

5. Crego-Calama, M.; Reinhoudt, D. N. New Materials for Metal Ion Sensing by Self-Assembled Monolayers on Glass. Adv. Mater. 2001, 13, 1171-1174. 
6. Piner, R. D.; Zhu, J.; Xu, F.; Hong, S. H.; Mirkin, C. A. "Dip-Pen" Nanolithography. Science 1999, 283, 661-663.

7. Ginger, D. S.; Zhang, H.; Mirkin, C. A. The Evolution of Dip-Pen Nanolithography. Angew. Chem., Int. Ed. 2004, 43, 30-45.

8. Salaita, K.; Wang, Y. H.; Mirkin, C. A. Applications of Dip-Pen Nanolithography. Nat. Nanotechnol. 2007, 2, 145-155.

9. Kumar, A.; Whitesides, G. M. Features of Gold Having Micrometer to Centimeter Dimensions Can Be Formed through a Combination of Stamping with an Elastomeric Stamp and an Alkanethiol Ink Followed by Chemical Etching. Appl. Phys. Lett. 1993, 63, 2002-2004.

10. Kassies, R.; Van der Werf, K. O.; Lenferink, A.; Hunter, C. N.; Olsen, J. D.; Subramaniam, V.; Otto, C. Combined Afm and Confocal Fluorescence Microscope for Applications in Bio-Nanotechnology. $J$. Microsc 2005, 217, 109-116.

11. Kassies, R. Atomic Force Fluorescence Microscopy. University of Twente, 2005.

12. Weeks, B. L.; Noy, A.; Miller, A. E.; De Yoreo, J. J. Effect of Dissolution Kinetics on Feature Size in Dip-Pen Nanolithography. Phys. Rev. Lett. 2002, 88, 255505.

13. Jang, J. Y.; Schatz, G. C.; Ratner, M. A. Liquid Meniscus Condensation in Dip-Pen Nanolithography. J. Chem. Phys. 2002, 116, 3875-3886.

14. Peterson, E. J.; Weeks, B. L.; De Yoreo, J. J.; Schwartz, P. V. Effect of Environmental Conditions on Dip Pen Nanolithography of Mercaptohexadecanoic Acid. J. Phys. Chem. B 2004, 108, 15206-15210.

15. Weeks, B. L.; Vaughn, M. W.; DeYoreo, J. J. Direct Imaging of Meniscus Formation in Atomic Force Microscopy Using Environmental Scanning Electron Microscopy. Langmuir 2005, 21, 8096-8098.

16. Nafday, O. A.; Weeks, B. L. Relative Humidity Effects in Dip-Pen Nanolithography of Alkanethiol Mixtures. Langmuir 2006, 22, 10912-10914.

17. Wang, W. C. M.; Stoltenberg, R. M.; Liu, S. H.; Bao, Z. N. Direct Patterning of Gold Nanoparticles Using Dip-Pen Nanolithography. Acs Nano 2008, 2, 2135-2142.

18. Maxwell, J. M.; Huson, M. G. Using the Scanning Probe Microscope to Measure the Effect of Relative Humidity on Sample Stiffness. Rev. Sci. Instrum. 2002, 73, 3520-3524.

19. Stukalov, O.; Murray, C. A.; Jacina, A.; Dutcher, J. R. Relative Humidity Control for Atomic Force Microscopes. Rev. Sci. Instrum. 2006, 77, 033704.

20. Basabe-Desmonts, L.; Beld, J.; Zimmerman, R. S.; Hernando, J.; Mela, P.; Parajo, M. F. G.; van Hulst, N. F.; van den Berg, A.; Reinhoudt, D. N.; Crego-Calama, M. A Simple Approach to Sensor Discovery and Fabrication on Self-Assembled Monolayers on Glass. J. Am. Chem. Soc. 2004, 126, 7293-7299.

21. Su, M.; Pan, Z. X.; Dravid, V. P.; Thundat, T. Locally Enhanced Relative Humidity for Scanning Probe Nanolithography. Langmuir 2005, 21, 10902-10906.

22. Haaheim, J.; Eby, R.; Nelson, M.; Fragala, J.; Rosner, B.; Zhang, H.; Athas, G. Dip Pen Nanolithography (Dpn): Process and Instrument Performance with Nanoink's Nscriptor System. Ultramicroscopy 2005, 103, 117-132. 


\title{
Chapter 4
}

\section{Protein Immobilization on Ni(II) Ion Patterns Prepared by Microcontact Printing and Dip-Pen Nanolithography*}

\begin{abstract}
An indirect method of protein patterning by using Ni(II) ion templates for immobilization via a specific metal-protein interaction is described. A nitrilotriacetic acid (NTA)-terminated monolayer allows oriented binding of histidine-tagged proteins via complexation with late first-row transition metal ions, such as $\mathrm{Ni}$ (II). Patterns of nickel(II) ions were prepared on NTA-terminated monolayer-functionalized glass slides by microcontact printing $(\mu \mathrm{CP})$ and dip-pen nanolithography (DPN) to obtain micrometer and submicrometer scale patterns. Consecutive dipping of the slides in $\mathrm{His}_{6}$-protein solutions resulted in the formation of protein patterns, as was subsequently proven by AFM and confocal fluorescence microscopy. This indirect method prevents denaturation of fragile biomolecules caused by direct printing or writing of proteins. Moreover, it yields well-defined patterned monolayers of proteins and, in principle, is well suited for biomolecules with a high molecular weight. This approach also enabled us to characterize the dependence of transfer of $\mathrm{Ni}$ (II) ions on fundamental parameters of DPN, such as writing speeds and tip-surface contact times.
\end{abstract}

*Part of this chapter has been published in Wu, C. C.; Reinhoudt, D. N.; Otto, C.; Velders, A. H.; Subramaniam, V. ACS Nano 2010, 4, 1083-1091. 


\subsection{Introduction}

Micro- and nanopatterning of biomolecules on surfaces attracts growing attention due to the potential applications in medicine and biotechnology. ${ }^{1}$ Applications include production and preparation of biochips and biosensors for diagnostics, drug discovery, and fundamental studies of biological processes. ${ }^{1,2}$ Different techniques have been developed to accomplish biopatterning at the micro- and nanoscale, such as soft lithography, ${ }^{3-5}$ scanning probe lithography, ${ }^{6-11}$ nanoimprint lithography, ${ }^{12-14}$ and electron beam lithography. ${ }^{15-17}$

In Chapter 3, it had been demonstrated that metal ions can be transferred onto fluorescent monolayers using microcontact printing $(\mu \mathrm{CP})$ and dip-pen nanolithography (DPN) with micrometer and sub-micrometer precision. ${ }^{18}$ Subsequent modulations of fluorescent signals were visualized in situ by using a hybrid atomic force fluorescence microscope (AFFM). ${ }^{18,19}$ A potential application of metal-ion patterns is their use as a template for selective and controlled immobilization of biomolecules via a specific metal-protein interaction. ${ }^{20,21}$

Nickel(II)-nitrilotriacetic acid (Ni-NTA) was originally developed in immobilized metal affinity chromatography (IMAC) for purifying proteins labeled with a short sequence of histidines (His-tagged proteins). ${ }^{22}$ NTA is a tetradentate ligand which can form a hexagonal complex with $\mathrm{Ni}$ (II) ions (or other divalent metal ions such as $\mathrm{Cu}$ (II) or $\mathrm{Co}(\mathrm{II})$. This way, four of six binding sites are occupied by the metal ion and the other two binding positions are available for histidines. The affinity of the hexahistidine $\left(\mathrm{His}_{6}\right)$ to Ni-NTA is high $\left(\mathrm{K}_{\mathrm{d}} \approx 10^{-13} \mathrm{M}\right){ }^{23}$ but the complexation of $\mathrm{His}_{6}$ to Ni-NTA can also be reversed by adding imidazole or EDTA. ${ }^{12,} 24$ These characteristics make the Ni-NTA system a popular choice for fabricating oriented and reversible His-tagged biomolecule patterns on surfaces. ${ }^{8,11,12,24}$ The Ni-NTA-His-tag system is thus a suitable candidate to prove our concept of using metal-ion patterns as templates for the immobilization of biomolecules.

In this chapter, the use of microcontact printing $(\mu \mathrm{CP})$ to fabricate $\mu \mathrm{m}$ scale $\mathrm{Ni}(\mathrm{II})$ ion patterns onto NTA-terminated monolayer-functionalized glass slides is first described because of the simplicity and high-throughput production of the $\mu \mathrm{CP}$ technique. ${ }^{3}$ The metal-ion patterned substrates were incubated immediately with His-tagged protein solutions to anchor the proteins onto the printed areas. Subsequently, the AFFM was utilized to perform DPN to scale down the size of Ni(II) ion patterns to the sub- $\mu \mathrm{m}$ scale, accompanied by direct visualization of the results by atomic force microscopy (AFM) and 
confocal fluorescence microscopy. The procedure of fabricating protein patterns is illustrated in Scheme 4.1.

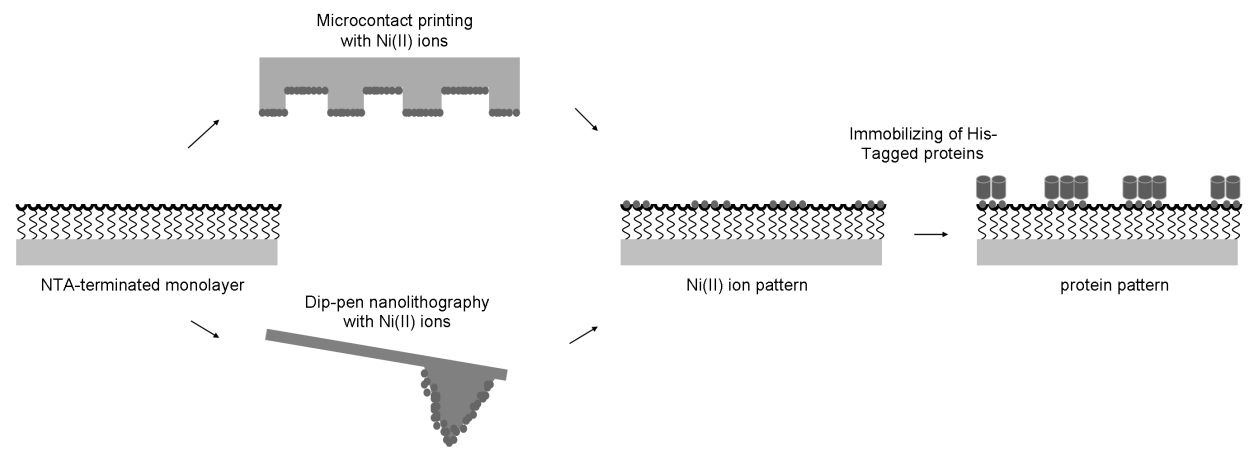

Scheme 4.1. Fabrication of Ni(II)-ion patterns onto NTA monolayer with microcontact printing $(\mu C P)$ and dip-pen nanolithography (DPN) to immobilize His-tagged proteins.

Using metal ion patterns as a template is an indirect approach to fabricate protein patterns, with the advantage that denaturation of fragile biomolecules readily observed when printing or writing proteins directly is avoided. ${ }^{25}$ Enhanced green fluorescent protein incorporating a $\mathrm{His}_{6}$-tag (His-EGFP) was used as a model protein. EGFP is intrinsically fluorescent when properly folded and thus can be directly used for optical recognition. The intrinsic fluorescence is also a marker of the structural integrity of the protein; misfolded or otherwise structurally damaged proteins do not fluoresce. The AFFM enables both fabrication of $\mathrm{Ni}$ (II) ion patterns and in situ observation of the fluorescence and AFM topography of the protein patterns. AFM measurements provide a better determination of the actual size of protein patterns as they surpass the resolution limitation of confocal fluorescence microscopy. Despite the difference in sizes of the proteins and the intermediate metal ions, fluorescence and topographic images of the protein patterns enabled us to quantitatively characterize the transfer and diffusion on the surface of Ni(II) ions as a function of fundamental parameters of DPN, such as writing speeds and tip-surface contact times. In a further demonstration of the general applicability of this method, a higher molecular weight histidine-tagged protein, the tetrameric reef coral fluorescent protein (His-DsRed), has also been used for patterning. 


\subsection{Results and Discussion}

\subsubsection{Ni-NTA system}

The Ni-NTA monolayers were prepared following the procedures reported by Paik et $a{ }^{26}$ The approach starts with the formation of epoxy-terminated monolayers of 3-glycidyloxypropyl trimethoxysilane on cleaned microscope glass slides. The epoxy groups were subsequently reacted with $N, N$-bis(carboxymethyl)-L-lysine to form NTA-terminated monolayers. Ni-NTA monolayers were obtained by immersing NTA-functionalized substrates into $\mathrm{NiCl}_{2}$ solution for the complexation of metal ions. The process of Ni-NTA monolayer preparation is depicted in Scheme 4.2. The formation of the monolayers was examined by water contact-angle goniometry and ellipsometry. The results are consistent with the values reported in literature. ${ }^{12,27}$

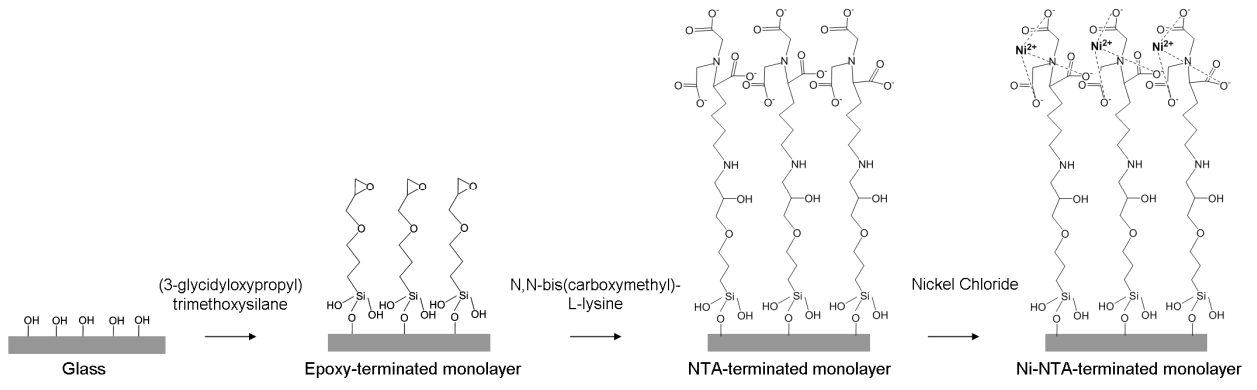

Scheme 4.2. Illustration of the preparation procedure for Ni-NTA-terminated monolayers.

In order to confirm that there is sufficient fluorescence contrast for further $\mu \mathrm{CP}$ and DPN experiments, $\sim 20 \mu \mathrm{l}$ of $100 \mathrm{nM}$ His-EGFP solution was placed onto NTA and Ni-NTA-functionalized surfaces for 30 minutes, which then were rinsed with $10 \mathrm{mM}$ Tris- $\mathrm{HCl}$ buffer ( $\mathrm{pH}$ 8.0) and Milli-Q water before drying with $\mathrm{N}_{2}$. The background-subtracted fluorescence images on NTA and Ni-NTA-functionalized surfaces obtained by AFFM are shown in Figure 4.1a and 4.1b, respectively. Non-specific binding of His-EGFP on the NTA-terminated monolayer was observed. The average fluorescence intensity values (a.u.) of Figure 4.1a and 4.1b, plotted in Figure 4.1c, are 1.26 and 14.02, respectively, yielding a ratio of average fluorescence intensity of the specifically-bound protein to the unspecifically bound protein of $\sim 11: 1$, which is a sufficiently large fluorescence contrast to perform $\mu \mathrm{CP}$ and DPN experiments. 
a)

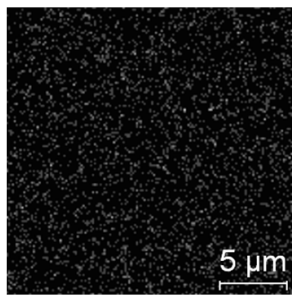

b)

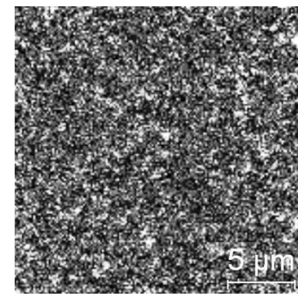

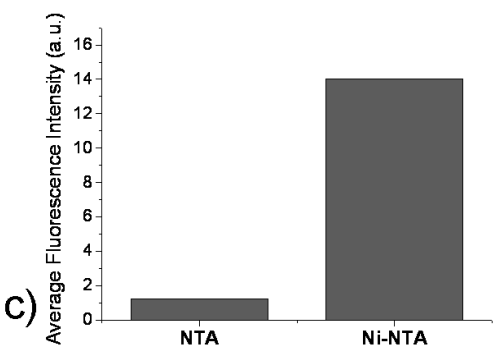

Figure 4.1. Fluorescence image of His-EGFP attached on (a) NTA- and (b) Ni-NTA-terminated monolayer-functionalized surface. The exposure time of the images is $0.25 \mathrm{~ms}$ per pixel. (c) The bars in the graph indicate the average fluorescence intensity of (a) and (b).

\subsubsection{Fabrication of His-EGFP patterns by microcontact printing ( $\mu \mathrm{CP})$}

Microcontact printing $(\mu \mathrm{CP})$ is an easy and fast approach to prove that $\mathrm{Ni}(\mathrm{II})$ ion patterns on NTA-terminated monolayer-functionalized surfaces can be used as templates for the immobilization of His-tagged proteins at $\mu \mathrm{m}$ scale. A PDMS stamp with $5 \mu \mathrm{m}$ line features with a spacing of $3 \mu \mathrm{m}$ was coated with $\mathrm{Ni}(\mathrm{II})$ ions and brought into conformal contact with the NTA-modified surface for 3 minutes before incubating the latter in a 100 nM His-EGFP solution. In Figure 4.2a, the fluorescence image of a patterned surface shows His-EGFP functionalized areas with the expected feature size of the microcontact printed Ni(II) ion pattern, illustrating that His-EGFP patterns can be readily obtained by this indirect method of using $\mathrm{Ni}$ (II) ions as templates. It has been previously reported that His-tagged proteins complexed on Ni(II)-NTA-terminated monolayers can be easily removed by an EDTA solution for reusing the functionalized substrates. ${ }^{12}$ To demonstrate that our functionalized surfaces are reusable, a substrate with a His-EGFP pattern was incubated in a $0.1 \mathrm{M}$ EDTA solution overnight and then rinsed with water and $10 \mathrm{mM}$ Tris-HCl buffer ( $\mathrm{pH} 8$ ). Figure $4.2 \mathrm{~b}$ shows that there are no apparent fluorescent features on the substrate after treatment with EDTA solution. A PDMS stamp with a different feature size (10 $\mu \mathrm{m}$ line features, separated by $5 \mu \mathrm{m})$ was used for a second microcontact printing step of $\mathrm{Ni}(\mathrm{II})$ ions on the regenerated slide. Figure 4.2c shows that His-EGFP was successfully immobilized on these surfaces, confirming that the NTA-functionalized surface is robust and can be reutilized. The slight difference in fluorescence intensities 
between panels a and c of Figures 4.2 may result from the difference in the amount of $\mathrm{Ni}(\mathrm{II})$ ions adsorbed onto the surfaces of the different PDMS stamps used.

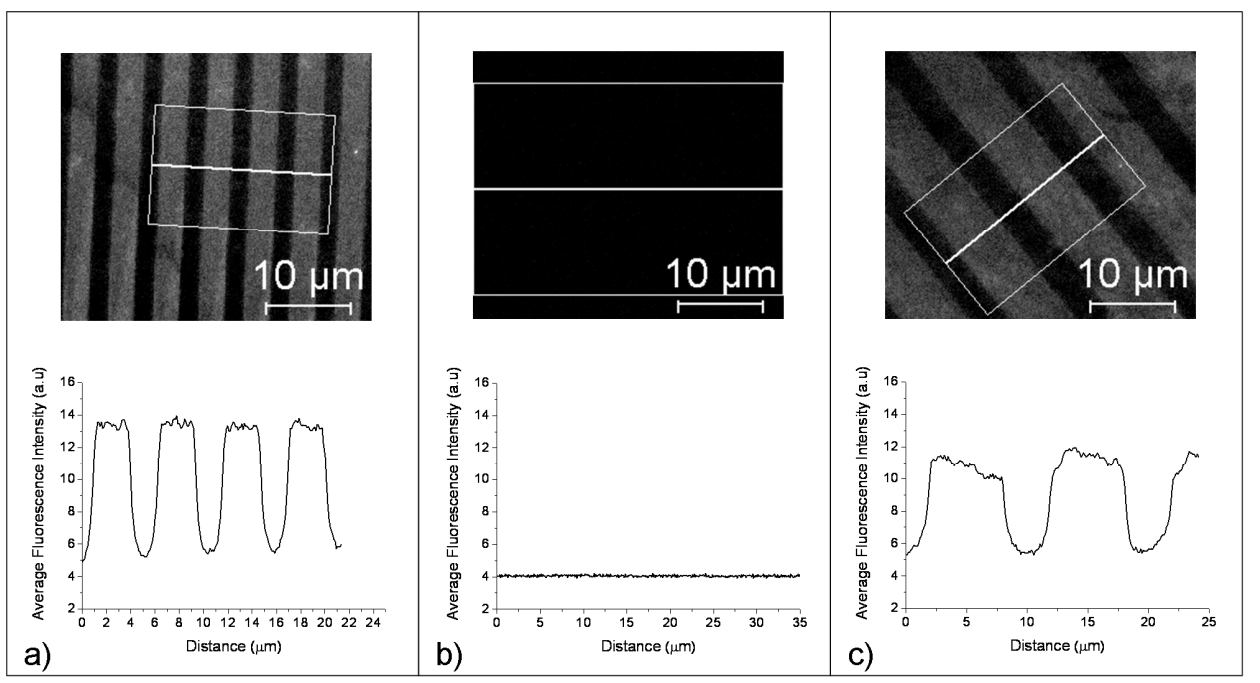

Figure 4.2. Fluorescence images and average fluorescence intensity profiles of the area inside the rectangle of His-EGFP immobilized on Ni(II) patterns created after (a) $1^{\text {st }} \mu C P$ of $5 \mu \mathrm{m}$ line features, (b) after rinsing with $0.1 \mathrm{M}$ EDTA solution and (c) after $2^{\text {nd }} \mu \mathrm{CP}$ of $10 \mu \mathrm{m}$ line features. The exposure time of the camera for each of the images is $200 \mathrm{~ms}$.

\subsubsection{Fabrication of His-EGFP patterns by dip-pen nanolithography (DPN)}

The fabrication of sub- $\mu \mathrm{m}$ scale metal ion patterns on NTA monolayer-functionalized glass was explored by DPN, a technique which is more flexible and can create patterns with smaller feature sizes than $\mu \mathrm{CP} .{ }^{28} \mathrm{DPN}$ can also modify the surface with different inks whereas $\mu \mathrm{CP}$ is normally a single ink process. The metal-ion patterns were fabricated with DPN, followed by protein incubation, and subsequently monitored by fluorescence spectroscopy and AFM topography in situ using the AFFM. The use of this hybrid instrument avoids the intrinsic difficulty of finding the written protein patterns after transferring the sample from the AFM to a fluorescence microscope. For the purpose of scaling down the size of $\mathrm{Ni}$ (II) ion patterns, a cleaned $\mathrm{Si}_{3} \mathrm{~N}_{4}$ AFM tip was inked with $\mathrm{Ni}(\mathrm{II})$ ions by dipping into a $\mathrm{NiCl}_{2}$ solution, and the ink-loaded tip was brought into contact with 
an NTA-functionalized surface for writing dot or line patterns directly above the central focus of the objective lens. The driving forces for the transfer of Ni(II) ions from an AFM tip to a surface are the dissolution of metal ions into the aqueous meniscus formed between tip and surface and the complex formation of Ni(II) ions with NTA molecules functionalized on the glass substrates. The written $\mathrm{Ni}$ (II) ion patterns were then immersed in a protein solution and consecutively visualized by fluorescence microscopy.

Metal ions are among the smallest ink "molecules" one can use. It is very difficult to directly characterize the transferred $\mathrm{Ni}$ (II) ions on a surface because of the small size of the ions. However, the amount of transfer of $\mathrm{Ni}$ (II) ions can be revealed by the subsequent immobilization of His-EGFP and measurement of its intrinsic fluorescence, allowing the systematic investigation of metal-ion deposition by DPN. Therefore, DPN experiments were carried out in which either an ink-coated AFM tip was held at different fixed positions for different times (dot patterns), or in which the AFM tip was moved at different, but constant, writing speeds (line patterns).

\subsubsection{Writing with different contact times}

A Ni(II) ion pattern of 18 dots was written with different tip-surface contact times for each dot, varying between 1 and $60 \mathrm{~s}$. A commercial $\mathrm{Si}_{3} \mathrm{~N}_{4}$ AFM tip was incubated into 10 $\mathrm{mM}$ Tris- $\mathrm{HCl}$ buffer ( $\mathrm{pH} 8.0$ ) containing $0.1 \mathrm{M} \mathrm{NiCl}_{2}$ for coating $\mathrm{Ni}(\mathrm{II})$ ions and then blown dry with $\mathrm{N}_{2}$. Subsequently, the ink-coated tip was brought into contact with the surface for a given time and was retracted after writing each dot. The contact time was monotonically increased, and the whole writing sequence was completed without reinking the tip. The written area was immediately incubated in a $100 \mathrm{nM}$ His-EGFP solution for 30 minutes and then rinsed with $10 \mathrm{mM}$ Tris- $\mathrm{HCl}$ buffer $(\mathrm{pH} 8)$ and Milli-Q water before drying with $\mathrm{N}_{2}$. The fluorescence image of the His-EGFP dot patterns is shown in Figure 4.3a. The observed His-EGFP dot patterns are consistent with the written $\mathrm{Ni}$ (II) ion patterns, which indicates that $\mathrm{Ni}$ (II) ions have been transferred from the AFM tip onto the surface. To elucidate the relationship between the fluorescence intensity and tip-surface contact time, the fluorescence intensities of a 32x32 pixel area covering each dot were averaged. It is known that there is a correlation between the tip-substrate contact time and the amount of deposited ink molecules. ${ }^{9,28,29}$ Normally, longer contact times between the tip and the substrate on a certain area, or using slower scanning speeds, should result in higher amount of ink transferred to the substrate. In Figure 4.3a, dots deposited for longer 
times show higher fluorescence intensity, indicating that more His-EGFP molecules were attached to the written area. In figure $4.3 \mathrm{~b}$, a plot of the averaged fluorescence intensities versus the tip-surface contact time shows a linear relationship, suggesting that the amount of Ni(II) ions transferred onto the NTA-functionalized surface increases linearly with the tip-surface contact time.

One important factor in determining the resolution in DPN experiments is how ink molecules diffuse from a tip to a surface via a water meniscus. ${ }^{30}$ Our DPN experiments were carried out at $\sim 60 \%$ relative humidity $(\mathrm{RH})$, and it has been reported that, at this $\mathrm{RH}$, a water meniscus of $\sim 1.5 \mu \mathrm{m}$ in diameter is formed between tip and substrate. ${ }^{31}$ The size of the water meniscus may suggest that the maximum size of the $\mathrm{Ni}$ (II) ion spot and its corresponding His-EGFP pattern is also $~ 1.5 \mu \mathrm{m}$ in diameter. ${ }^{31}$ The diameters of His-EGFP dot patterns written with tip-surface contact times longer than 10 seconds shown in Figure 4.3a are in fact between $\sim 1.0$ and $\sim 1.4 \mu \mathrm{m}$. Besides, a gradient in fluorescence intensity was observed; that is each dot exhibits higher emission in the center and a lower emission at the borders; one example is exhibited in Figure 4.3c, which is the enlargement of the fluorescent dot pattern written with a tip-surface contact time of $60 \mathrm{~s}$. Figure $4.3 \mathrm{~d}$ shows the average fluorescence intensity profile of the area inside the rectangle as indicated in Figure 4.3c, and clearly highlights the gradient of fluorescence intensity. This profile can be fit well with a Gaussian function, which suggests that the gradient phenomenon may be attributed to the radial diffusion of the nickel ions from the contact position of the tip and surface to the border of the spot.

AFM imaging reveals topographic information of the protein patterns adhered to metal-ion sites. To avoid contaminations, a new $\mathrm{Si}_{3} \mathrm{~N}_{4}$ AFM tip was brought to the original position in the DPN experiments, directly above the central focus of the objective lens. The topography measurements were performed in tapping mode and are shown in Figure 4.4a. The 18-dots protein pattern shown in the AFM height image corresponds well to the pattern in the fluorescence image. It can be observed that the spots with higher fluorescence intensity have more distinct AFM patterns, suggesting the denser packing of His-EGFP. The diameters of these more densely packed protein dots are between $\sim 300$ $\mathrm{nm}$ and $\sim 800 \mathrm{~nm}$. The height profile of protein patterns generated with tip-surface contact times of $10 \mathrm{~s}, 28 \mathrm{~s}$ and $48 \mathrm{~s}$ is shown in Figure 4.4b. The reported size of EGFP in literature is $\sim 2 \mathrm{~nm}$ in width and $\sim 4 \mathrm{~nm}$ in height. ${ }^{32}$ The data in Figure $4.4 \mathrm{~b}$ thus suggest that a monolayer of patterned proteins was achieved. 
a)

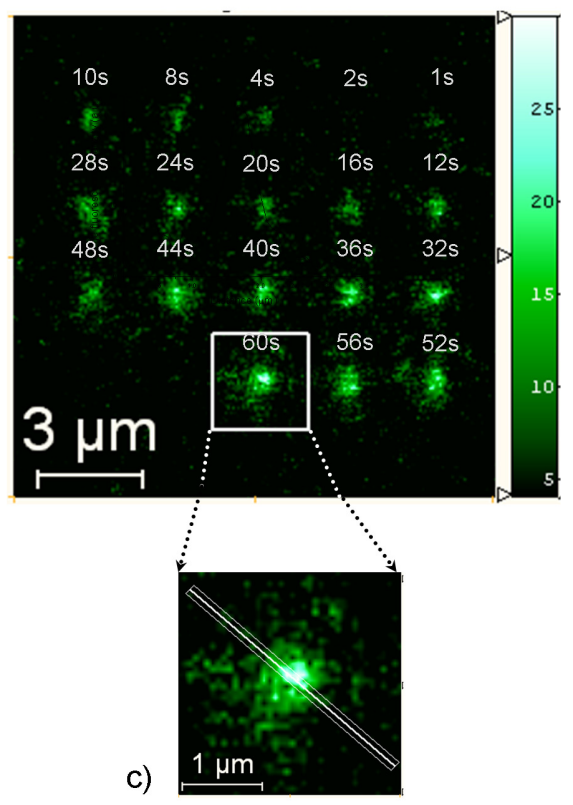

b)

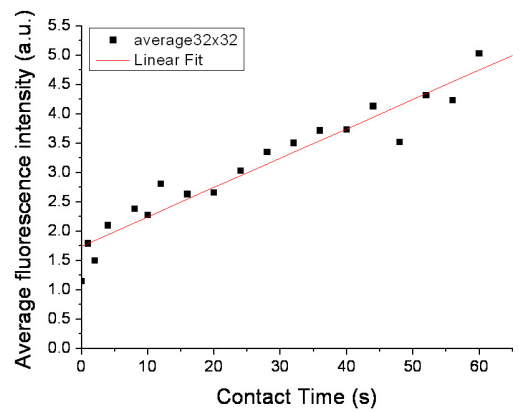

d)

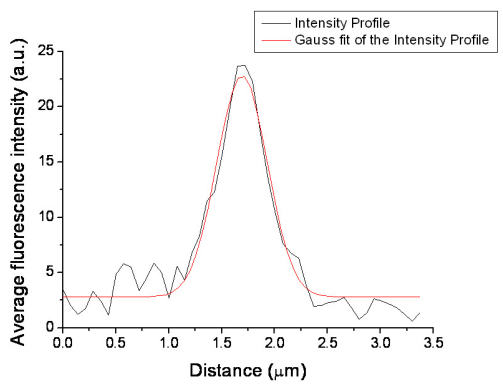

Figure 4.3. (a) Fluorescence images of His-EGFP patterns acquired after incubating slides, with deposited metal ions areas, with the protein solution. The dot patterns were generated by using an AFM tip inked with Ni(II) ion using tip-surface contact times listed above the dot patterns. The exposure time used for obtaining the images was $0.25 \mathrm{~ms}$ per pixel. (b) Plot of average fluorescence intensity of each dot within a $32 \times 32$ pixel region around the dot versus tip-surface contact time. The intensity with a contact time of 0 second was obtained by averaging a 32x32 pixel area which had not been in contact with the ink-coated tip; the observed fluorescence can be attributed to the non-specific binding effect of protein on the NTA functionalized surface. A linear fit to the data is plotted in red. (c) Zoom-in of the His-EGFP dot written with tip-surface contact time of 60 seconds shown in (a). (d) Average fluorescence intensity profile of the area inside the rectangle indicated in (c) (black line) and its Gaussian fit profile (red line). 
a)
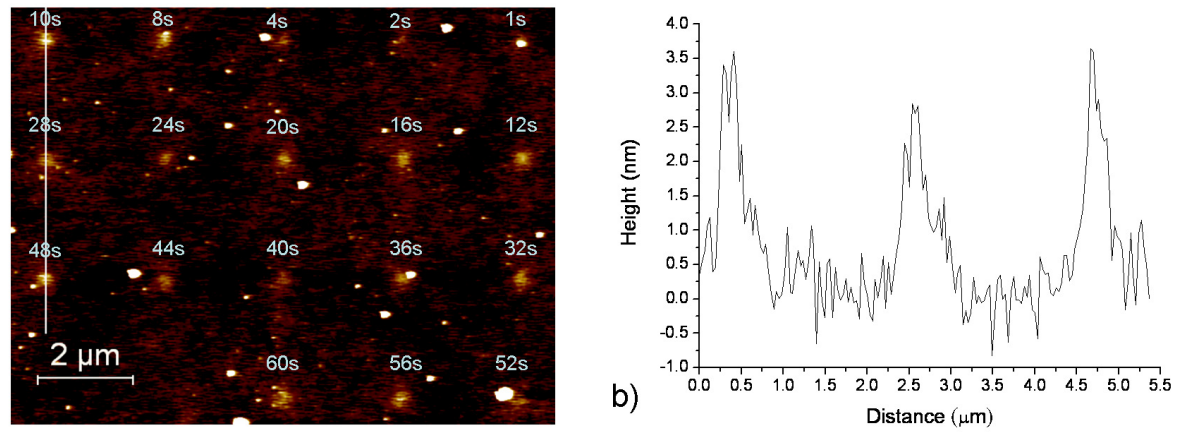

Figure 4.4. (a) AFM tapping mode height image of His-EGFP attached to Ni(II) ion patterns fabricated with dip-pen nanolithography. Some (white) spots shown in the image may result from dust particles or dried salt crystals from the buffer solution. (b) The height profile of protein dots generated with the tip-surface contact times of $10 \mathrm{~s}, 28 \mathrm{~s}$ and 48 s (from left to right).

\subsubsection{Writing at various speeds}

Also line features of His-EGFP were successfully generated by fabricating Ni(II) ion patterns as a template. A Ni(II) ion pattern of five $10 \mu \mathrm{m}$ long lines with five different scan speeds varying between $10 \mu \mathrm{m} / \mathrm{s}$ and $0.1 \mu \mathrm{m} / \mathrm{s}$ was written. Each line was scanned once, and the tip was retracted after finishing each line. The same tip was used without re-inking to fabricate a second, identical, 5-line pattern. The written area was incubated with a His-EGFP solution to develop the Ni(II) ion pattern. The fluorescence image of the fabricated His-EGFP lines is shown in Figure 4.5a. It was again observed that the protein line patterns correspond to the designed metal ion patterns. A gradient, in-plane and orthogonal to the writing direction, in the fluorescence intensity could also be observed in the line patterns, suggesting that a similar diffusion of $\mathrm{Ni}$ (II) ions takes place as observed in the dot patterning. Figure $4.5 \mathrm{~b}$ shows the average fluorescence intensity profiles of each line in the first 5-line pattern which were fit well with Gaussian functions. The lines with higher fluorescence intensity have more distinct AFM line patterns with a line width of $700 \mathrm{~nm}$. 

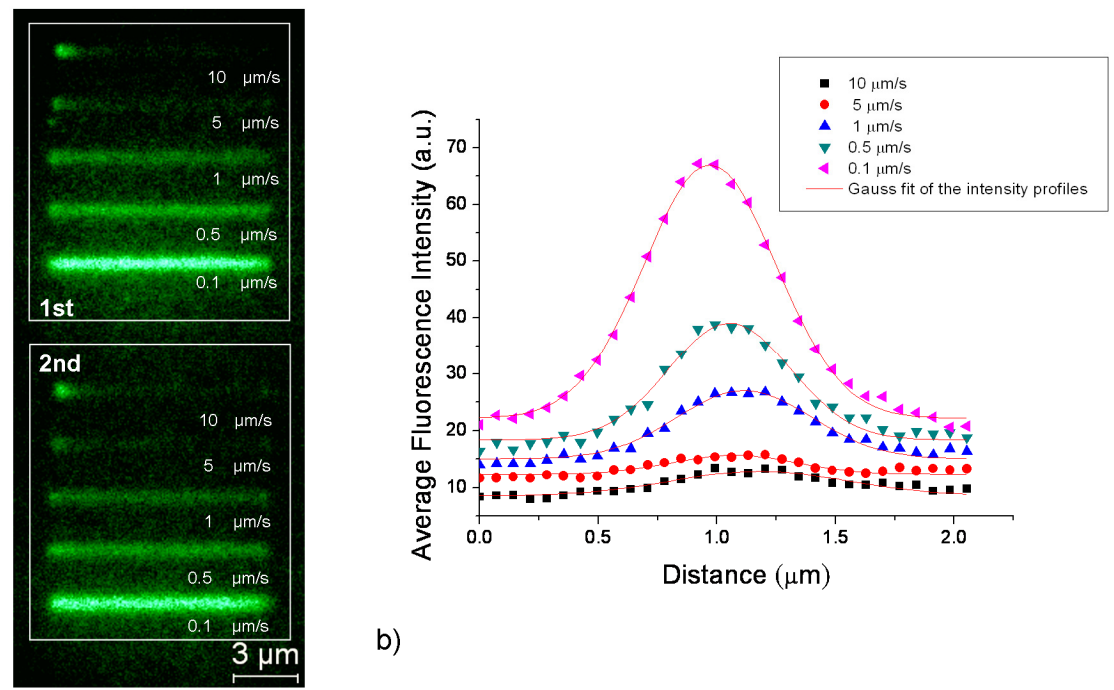

b)

Figure 4.5. (a) Fluorescence images of His-EGFP patterns acquired after incubating metal-ion written areas with protein solution. The upper 5-line pattern was written first.

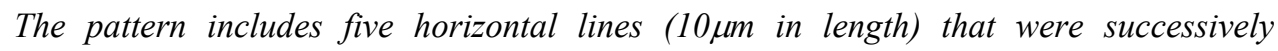
generated by using an AFM tip inked with Ni(II) ions, using scanning speeds of 10, 5, 1, 0.5 , and $0.1 \mu \mathrm{m} / \mathrm{s}$, from top to bottom. A second 5-lines pattern was generated by using the same tip without re-inking. The camera exposure time for these images is $0.25 \mathrm{~ms}$ per pixel. (b) Average fluorescence intensity profiles and Gaussian fit (in red) of each line in $1^{\text {st }}$ 5-line pattern.

The relationship between the amount of deposited Ni(II) ions and scanning speeds derived by measuring the average fluorescence intensity of each line versus writing speed is shown in Figure 4.6a. Comparing the average fluorescence intensities of these two 5-line patterns, we note that the lines written with lower speeds in the first 5-line pattern have slightly higher fluorescence intensity than the ones in the second 5-line pattern. This decrease of fluorescence intensity might result from the depletion of $\mathrm{Ni}$ (II) ions coated on the AFM tip surface while performing DPN experiments continuously, that is, without reinking. From Figure 4.5 and $4.6 \mathrm{a}$, it is clear that Ni(II) ion lines written at a lower scanning rate show higher fluorescence intensity, which indicates that more His-EGFP molecules were attached to the written area. To convert the relation between the 
fluorescence intensity versus writing speed to the fluorescence intensity versus tip-surface contact time, the fluorescence intensities over the area of each line (142 x 14 pixels) were averaged. The tip-surface contact time is the time taken to finish the scan of one line. Figure $4.6 \mathrm{~b}$ shows the average fluorescence intensity versus tip-surface contact time of these two 5-line patterns. A close to saturation fluorescence intensity was observed while depositing Ni(II) ions with the slowest scanning speed. A similar result has been reported by Deng et al., observing the saturation of fluorescence emitted from $\mathrm{His}_{6}$-GFP when the $\mathrm{Cu}$ (II) ion density on polyether-functionalized glass substrates is more than $\sim 1.5 \mathrm{x} 10^{14}$ $\mathrm{cm}^{-2} \cdot 33$

It is interesting to notice the difference between Figure 4.3b (steady state) and Figure $4.6 \mathrm{~b}$ (writing), although the values of the fluorescence intensity in these two figures are not easily compared with each other due to different experimental conditions. Figure $4.6 \mathrm{~b}$ shows that the average fluorescence intensity saturates gradually, while the average fluorescence intensity in Figure $4.3 \mathrm{~b}$ does not show a tendency to saturate even after depositing $\mathrm{Ni}(\mathrm{II})$ ions for 60 seconds. This difference is probably due to the different amounts of $\mathrm{Ni}$ (II) ions deposited onto the tip surface at the beginning of DPN experiments. There was a practical difficulty to make tips be coated with the same amount of Ni(II) ions. To allow a more thorough investigation of the fundamental DPN parameters, it would be useful to overcome the ink depletion and imperfect coating of ink molecules, for example, by exploiting fountain pen nanolithography ${ }^{34}$ or functionalized tip surfaces ${ }^{35,36}$ as an alternative approach for future experiments.

a)

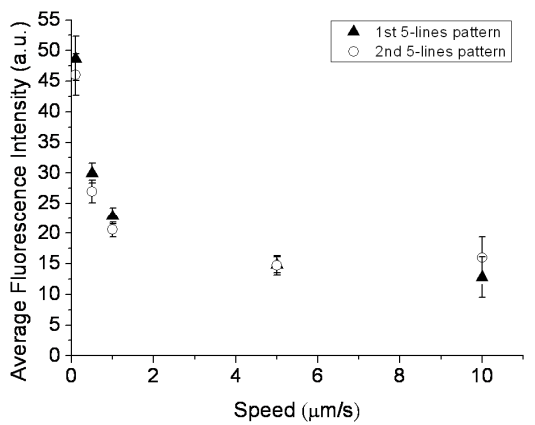

b)

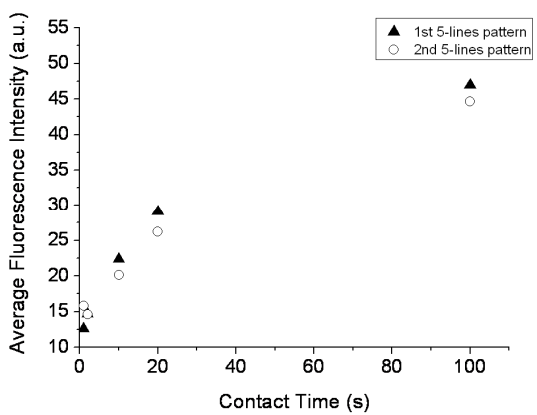

Figure 4.6. (a) Average fluorescence intensities of each line in Fig. 4.5a versus the writing speeds. (b) Plot of average fluorescence intensity of each line within $142 x 14$ pixels $(\sim 10 \mu \mathrm{m}$ in length and $\sim 1 \mu \mathrm{m}$ in width) in Fig. 4.5 a versus tip-surface contact time. 


\subsubsection{Fabrication of His-DsRed patterns by $\mu C P$ and DPN}

Monomeric His-EGFP was used to demonstrate successfully that proteins could be immobilized onto metal-ion templates fabricated with $\mu \mathrm{CP}$ and DPN. To further demonstrate the general applicability of this method, the tetrameric reef coral fluorescent protein incorporating a His-tag (His-DsRed) was also used for patterning. Figure 4.7a and 4.7b show that His-DsRed was attached well onto the Ni(II) ion patterns fabricated by $\mu \mathrm{CP}$ and DPN, respectively. A tapping mode AFM topography image of the region displayed in Figure $4.7 \mathrm{~b}$ is shown in Figure $4.7 \mathrm{c}$. From the cross section profile of the AFM height image (Figure 4.7d), it can be seen clearly that the line width and height of His-DsRed line pattern is $\sim 400 \mathrm{~nm}$ and $\sim 4 \mathrm{~nm}$, respectively. The AFM topographic information again demonstrates the monolayer formation of proteins. In principle, this indirect writing method puts no limits regarding the molecular weight of the molecule (e.g., protein) to be immobilized, which offers an important flexibility and advantage as a patterning technique. Besides, this approach may provide the possibility of generating multiple protein patterns, for example, using EDTA to selectively remove some regions of pre-immobilized proteins and then back filling with another kind of His-tagged protein.

a)

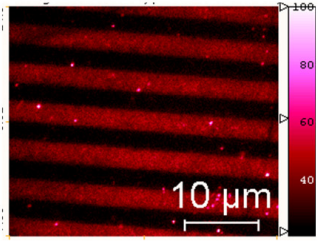

c)
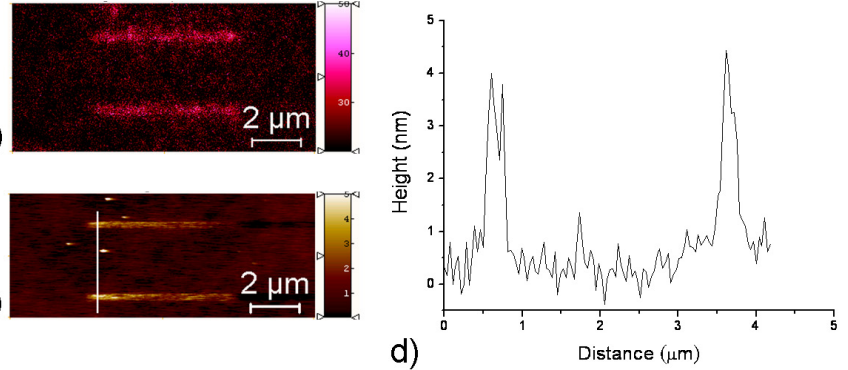

Figure 4.7. Fluorescence images of His-DsRed immobilized on Ni(II) ion pattern created by (a) microcontact printing and (b) dip-pen nanolithography. (c) AFM tapping mode height image of (b). (d) The cross section profile of His-DsRed line pattern shown in (c). 


\subsection{Conclusions}

In summary, a general method of fabricating Ni(II) ion patterns on NTA-terminated monolayer-functionalized glass substrates as templates has been developed for the immobilization of His-tagged biomolecules at micrometer and sub-micrometer scale by microcontact printing $(\mu \mathrm{CP})$ or dip-pen nanolithography (DPN). The advantage of this method is that it allows the oriented and reversible attachment of biomolecules while avoiding protein denaturation because no direct printing or writing of biomolecules is needed. Moreover, this method furnishes well-defined single-protein layers. The driving force for the transfer of $\mathrm{Ni}$ (II) ions from an AFM tip to a substrate is the dissolution of metal ions into the aqueous meniscus formed between tip and surface and the complex formation of Ni(II) ions with NTA molecules functionalized on the glass substrates.

The smallest size in diameter or width of His-EGFP or His-DsRed patterns that we can presently achieve by using DPN is around $400 \mathrm{~nm}$. This indirect approach may enable us to fabricate multiple protein patterns in the near future. We have used a hybrid atomic force and fluorescence microscope to perform direct write-read DPN experiments and obtained in situ fluorescence images and AFM topography information of the fabricated patterns. By utilizing the intrinsic fluorescence property of His-EGFP, we could study the relationship between tip-surface contact time and deposited amount of $\mathrm{Ni}$ (II) ions.

\subsection{Experimental}

\section{General procedures.}

All glassware used to prepare the monolayers was cleaned by sonicating for 60 minutes in a $2 \% \mathrm{v} / \mathrm{v}$ Hellmanex II solution in high purity water (Milli-Q, $18.2 \mathrm{M} \Omega \mathrm{cm}$ ), and then rinsed thoroughly with Milli-Q water, and dried in an ambient environment. Microscope glass slide substrates were cleaned in piranha solution for 20 minutes, (concentrated $\mathrm{H}_{2} \mathrm{SO}_{4}$ and $33 \%$ aqueous $\mathrm{H}_{2} \mathrm{O}_{2}$ in a $3: 1$ ratio). [Warning: Piranha solution should be handled with caution: it has been reported to detonate unexpectedly.] The glass slides were then rinsed several times with Milli-Q water, and dried gently under a nitrogen stream before forming the monolayer.

\section{NTA and Ni-NTA monolayer preparation. ${ }^{26}$}

The freshly cleaned substrates were immersed into a distilled toluene solution containing $1 \%$ (vol/vol) 3-glycidyloxypropyl trimethoxysilane under argon for two days. After the substrates were removed from the solution, they were rinsed with distilled toluene and dried under a nitrogen stream. The substrates functionalized with epoxy-terminated monolayer were incubated in $10 \mathrm{mM}$ Tris- $\mathrm{HCl}$ 


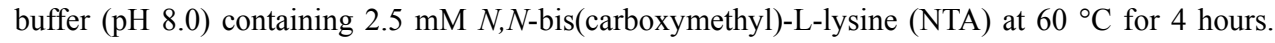
The substrates were rinsed with Milli-Q water and dried in preparation for $\mu \mathrm{CP}$ and DPN experiments. Ni-NTA surfaces were obtained by immersing the NTA-functionalized substrates into $10 \mathrm{mM}$ Tris-HCl buffer ( $\mathrm{pH}$ 8.0) containing $0.1 \mathrm{M} \mathrm{NiCl}_{2}$ for 30 minutes. They were then rinsed several times with Milli-Q water, and dried under a nitrogen stream. The formation of the monolayers was examined by water contact-angle goniometry and ellipsometry. The results are consistent with the values reported in literature. ${ }^{12,27}$

\section{Microcontact printing $(\mu \mathrm{CP})$.}

The preparation procedure of PDMS stamps was described in Chapter 3. The non-oxidized PDMS stamps with $5 \mu \mathrm{m}$ line features, with a spacing of $3 \mu \mathrm{m}$ and $10 \mu \mathrm{m}$ line features, with a spacing of 5 $\mu \mathrm{m}$ were incubated in $10 \mathrm{mM}$ Tris- $\mathrm{HCl}$ buffer ( $\mathrm{pH}$ 8.0) containing $0.1 \mathrm{M} \mathrm{NiCl}_{2}$ for $\sim 1$ hour and then dried with a nitrogen stream. The stamps were brought into contact with a NTA-terminated substrate for 3 minutes. After peeling off the stamp, the $\mathrm{Ni}(\mathrm{II})$ ions-printed substrates were incubated in $\sim 200 \mu \mathrm{l}$ of $25 \mathrm{mM}$ Tris- $\mathrm{HCl}$ buffer (pH 7.5) containing $100 \mathrm{nM}$ of His-EGFP or His-DsRed for 30 minutes and then rinsed with $10 \mathrm{mM}$ Tris-HCl buffer (pH 8.0) and Milli-Q water to remove excess protein.

\section{Acquisition of fluorescent images.}

The substrates with protein patterns were dried under a nitrogen stream before imaging with a fluorescence microscope (TE2000-U, Nikon) equipped with a mercury lamp as light source and a digital camera (Nikon digital Sight DS-Qi1Mc). The fluorescence images of His-EGFP and His-DsRed patterns were obtained by using filter cubes with blue excitation light $(\lambda=470 / 22 \mathrm{~nm}) /$ green emission light $(\lambda=520 / 35 \mathrm{~nm}) / \operatorname{dichroic}(\lambda=495 \mathrm{~nm})$ and green excitation light $(\lambda=510 / 40 \mathrm{~nm}) /$ red emission light $(\lambda=617 / 73 \mathrm{~nm}) /$ dichroic $(\lambda=562 \mathrm{~nm})$, respectively. The fluorescence images obtained were analyzed with the commercial software SPIP (Image Metrology, Version 4.4.3.0) and ImageJ (Version 1.40g).

\section{Dip pen nanolithography (DPN) and visualization with AFFM.}

A custom-built atomic force fluorescence microscope (AFFM) was used to perform dip-pen nanolithography and to observe the results of patterning in situ without having to change to another instrument. Commercial $\mathrm{Si}_{3} \mathrm{~N}_{4}$ AFM cantilevers (Veeco Probes) with nominal spring constant of $0.54 \mathrm{Nm}^{-1}$ were used. Immediately before a DPN experiment, the cantilever was rinsed rigorously with ethanol and dried under a stream of $\mathrm{N}_{2}$. Cleaned cantilevers were then immersed into $10 \mathrm{mM}$ Tris- $\mathrm{HCl}$ buffer $\left(\mathrm{pH} 8.0\right.$ ) containing $0.1 \mathrm{M} \mathrm{NiCl}_{2}$ for at least 15 minutes and dried with $\mathrm{N}_{2}$. The $\mathrm{Ni}$ (II) ions-coated cantilever was mounted on the AFM head and used to carry out DPN experiments on NTA-terminated monolayer-functionalized substrates in contact mode inside a custom-built chamber with a controlled humidity $(\sim 60 \%)$ at a temperature between 20 and $22{ }^{\circ} \mathrm{C} .{ }^{18,37}$ 
Immediately after DPN, $\sim 5 \mu \mathrm{l}$ of $100 \mathrm{nM}$ His-EGFP or His-DsRed solution was placed onto the written area for 30 minutes, and rinsed with $10 \mathrm{mM}$ Tris- $\mathrm{HCl}$ buffer $(\mathrm{pH} 8.0)$ and water to remove excess protein. Before fluorescence imaging of the DPN patterns, the substrate was carefully dried with a $\mathrm{N}_{2}$ stream in situ to avoid displacement of the patterned area from the observation range of the objective. The His-EGFP and His-DsRed patterns were excited with the $488 \mathrm{~nm}$ line of an argon-ion laser (163-D Laser System, Spectra Physics), and the emission of the fluorescence was recorded by an avalanche photodiode (APD) (SPCM-AQR-14, Perkin Elmer Optoelectronics). The original ink-coated tip was replaced by a new $\mathrm{Si}_{3} \mathrm{~N}_{4}$ AFM tip to prevent contamination of the patterned areas during subsequent imaging. AFM-tapping mode was used to obtain topographic information of the protein patterns. The images were obtained with 512 x 512 pixels acquisitions and scanning speeds of 1000 pixels per second. Fluorescence and topographic images were analyzed with the commercial software SPIP (Image Metrology, Version 4.4.3.0).

\subsection{Acknowledgements}

Dr. Martin Bennink and Kees van der Werf are acknowledged for their kind help with the modification of the AFM setup and Dr. Francesca Costantini for generously sharing her knowledge of preparing the monolayer.

\subsection{References}

1. Christman, K. L.; Enriquez-Rios, V. D.; Maynard, H. D. Nanopatterning Proteins and Peptides. Soft Matt. 2006, 2, 928-939.

2. Mendes, P. M.; Yeung, C. L.; Preece, J. A. Bio-Nanopatterning of Surfaces. Nanoscale Res. Lett. 2007, 2, 373-384.

3. Weibel, D. B.; DiLuzio, W. R.; Whitesides, G. M. Microfabrication Meets Microbiology. Nat. Rev. Microbiol. 2007, 5, 209-218.

4. Kane, R. S.; Takayama, S.; Ostuni, E.; Ingber, D. E.; Whitesides, G. M. Patterning Proteins and Cells Using Soft Lithography. Biomaterials 1999, 20, 2363-2376.

5. Li, H. W.; Muir, B. V. O.; Fichet, G.; Huck, W. T. S. Nanocontact Printing: A Route to Sub-50-Nm-Scale Chemical and Biological Patterning. Langmuir 2003, 19, 1963-1965.

6. Nam, J. M.; Han, S. W.; Lee, K. B.; Liu, X. G.; Ratner, M. A.; Mirkin, C. A. Bioactive Protein Nanoarrays on Nickel Oxide Surfaces Formed by Dip-Pen Nanolithography. Angew. Chem., Int. Ed. 2004, 43, 1246-1249.

7. Kenseth, J. R.; Harnisch, J. A.; Jones, V. W.; Porter, M. D. Investigation of Approaches for the Fabrication of Protein Patterns by Scanning Probe Lithography. Langmuir 2001, 17, 4105-4112.

8. Kim, K. H.; Kim, J. D.; Kim, Y. J.; Kong, S. H.; Jung, S. Y.; Jung, H. Protein Immobilization without Purification Via Dip-Pen Nanolithography. Small 2008, 4, 1089-1094. 
9. Ginger, D. S.; Zhang, H.; Mirkin, C. A. The Evolution of Dip-Pen Nanolithography. Angew. Chem., Int. Ed. 2004, 43, 30-45.

10. Salaita, K.; Wang, Y. H.; Mirkin, C. A. Applications of Dip-Pen Nanolithography. Nat. Nanotechnol. 2007, 2, 145-155.

11. Tinazli, A.; Piehler, J.; Beuttler, M.; Guckenberger, R.; Tampe, R. Native Protein Nanolithography That Can Write, Read and Erase. Nat. Nanotechnol. 2007, 2, 220-225.

12. Maury, P.; Escalante, M.; Peter, M.; Reinhoudt, D. N.; Subramaniam, V.; Huskens, J. Creating Nanopatterns of His-Tagged Proteins on Surfaces by Nanoimprint Lithography Using Specific Ninta-Histidine Interactions. Small 2007, 3, 1584-1592.

13. Falconnet, D.; Pasqui, D.; Park, S.; Eckert, R.; Schift, H.; Gobrecht, J.; Barbucci, R.; Textor, M. A Novel Approach to Produce Protein Nanopatterns by Combining Nanoimprint Lithography and Molecular Self-Assembly. Nano Lett. 2004, 4, 1909-1914.

14. Escalante, M.; Zhao, Y. P.; Ludden, M. J. W.; Vermeij, R.; Olsen, J. D.; Berenschot, E.; Hunter, C. N.; Huskens, J.; Subramaniam, V.; Otto, C. Nanometer Arrays of Functional Light Harvesting Antenna Complexes by Nanoimprint Lithography and Host-Guest Interactions. J. Am. Chem. Soc. 2008, 130, 8892-8893.

15. Hong, Y.; Krsko, P.; Libera, M. Protein Surface Patterning Using Nanoscale Peg Hydrogels. Langmuir 2004, 20, 11123-11126.

16. Rundqvist, J.; Hoh, J. H.; Haviland, D. B. Directed Immobilization of Protein-Coated Nanospheres to Nanometer-Scale Patterns Fabricated by Electron Beam Lithography of Poly(Ethylene Glycol) Self-Assembled Monolayers. Langmuir 2006, 22, 5100-5107.

17. Christman, K. L.; Schopf, E.; Broyer, R. M.; Li, R. C.; Chen, Y.; Maynard, H. D. Positioning Multiple Proteins at the Nanoscale with Electron Beam Cross-Linked Functional Polymers. J. Am. Chem. Soc. 2009, 131, 521-527.

18. Basabe-Desmonts, L.; Wu, C. C.; van der Werf, K. O.; Peter, M.; Bennink, M.; Otto, C.; Velders, A. H.; Reinhoudt, D. N.; Subramaniam, V.; Crego-Calama, M. Fabrication and Visualization of Metal-Ion Patterns on Glass by Dip-Pen Nanolithography. Chemphyschem 2008, 9, 1680-1687.

19. Kassies, R.; Van der Werf, K. O.; Lenferink, A.; Hunter, C. N.; Olsen, J. D.; Subramaniam, V.; Otto, C. Combined Afm and Confocal Fluorescence Microscope for Applications in Bio-Nanotechnology. $J$. Microsc. 2005, 217, 109-116.

20. Vega, R. A.; Maspoch, D.; Shen, C. K. F.; Kakkassery, J. J.; Chen, B. J.; Lamb, R. A.; Mirkin, C. A. Functional Antibody Arrays through Metal Ion-Affinity Templates. Chembiochem 2006, 7, 1653-1657.

21. Cha, T.; Guo, A.; Jun, Y.; Pei, D. Q.; Zhu, X. Y. Immobilization of Oriented Protein Molecules on Poly(Ethylene Glycol)-Coated Si(111). Proteomics 2004, 4, 1965-1976.

22. Porath, J.; Carlsson, J.; Olsson, I.; Belfrage, G. Metal Chelate Affinity Chromatography, a New Approach to Protein Fractionation. Nature 1975, 258, 598-599.

23. Paborsky, L. R.; Dunn, K. E.; Gibbs, C. S.; Dougherty, J. P. A Nickel Chelate Microtiter Plate Assay for Six Histidine-Containing Proteins. Anal. Biochem. 1996, 234, 60-65.

24. Ludden, M. L. W.; Mulder, A.; Schulze, K.; Subramaniam, V.; Tampe, R.; Huskens, J. Anchoring of Histidine-Tagged Proteins to Molecular Printboards: Self-Assembly, Thermodynamic Modeling, and Patterning. Chem. Eur. J. 2008, 14, 2044-2051. 
25. Lee, K. B.; Lim, J. H.; Mirkin, C. A. Protein Nanostructures Formed Via Direct-Write Dip-Pen Nanolithography. J. Am. Chem. Soc. 2003, 125, 5588-5589.

26. Paik, H. J.; Kim, Y. R.; Orth, R. N.; Ober, C. K.; Coates, G. W.; Batt, C. A. End-Functionalization of Poly(3-Hydroxybutyrate) Via Genetic Engineering for Solid Surface Modification. Chem. Commun. 2005, 1956-1958.

27. Luzinov, I.; Julthongpiput, D.; Liebmann-Vinson, A.; Cregger, T.; Foster, M. D.; Tsukruk, V. V. Epoxy-Terminated Self-Assembled Monolayers: Molecular Glues for Polymer Layers. Langmuir 2000, 16, 504-516.

28. Piner, R. D.; Zhu, J.; Xu, F.; Hong, S. H.; Mirkin, C. A. "Dip-Pen" Nanolithography. Science 1999, 283, 661-663.

29. Noy, A.; Miller, A. E.; Klare, J. E.; Weeks, B. L.; Woods, B. W.; DeYoreo, J. J. Fabrication of Luminescent Nanostructures and Polymer Nanowires Using Dip-Pen Nanolithography. Nano Lett. 2002, 2, 109-112.

30. Salazar, R. B.; Shovsky, A.; Schonherr, H.; Vancso, G. J. Dip-Pen Nanolithography on (Bio)Reactive Monolayer and Block-Copolymer Platforms: Deposition of Lines of Single Macromolecules. Small 2006, 2, 1274-1282.

31. Weeks, B. L.; Vaughn, M. W.; DeYoreo, J. J. Direct Imaging of Meniscus Formation in Atomic Force Microscopy Using Environmental Scanning Electron Microscopy. Langmuir 2005, 21, 8096-8098.

32. Tsien, R. Y. The Green Fluorescent Protein. Annual Review of Biochemistry 1998, 67, 509-544.

33. Deng, Y.; Zhu, X. Y.; Kienlen, T.; Guo, A. Transport at the Air/Water Interface Is the Reason for Rings in Protein Microarrays. J. Am. Chem. Soc. 2006, 128, 2768-2769.

34. Kim, K. H.; Sanedrin, R. G.; Ho, A. M.; Lee, S. W.; Moldovan, N.; Mirkin, C. A.; Espinosa, H. D. Direct Delivery and Submicrometer Patterning of DNA by a Nanofountain Probe. Adv. Mater. 2008, 20, 330-334.

35. Wu, C. C.; Xu, H. P.; Otto, C.; Reinhoudt, D. N.; Lammertink, R. G. H.; Huskens, J.; Subramaniam, V.; Velders, A. H. Porous Multilayer-Coated AFM Tips for Dip-Pen Nanolithography of Proteins. J. Am. Chem. Soc. 2009, 131, 7526-7527.

36. Senesi, A. J.; Rozkiewicz, D. I.; Reinhoudt, D. N.; Mirkin, C. A. Agarose-Assisted Dip-Pen Nanolithography of Oligonucleotides and Proteins. Acs Nano 2009, 3, 2394-2402.

37. Stukalov, O.; Murray, C. A.; Jacina, A.; Dutcher, J. R. Relative Humidity Control for Atomic Force Microscopes. Rev. Sci. Instrum, 2006, 77, 033704. 


\title{
Chapter 5
}

\section{Reactive Microcontact Printing and Dip-Pen Nanolithography: a strategy towards patterning submicrometer-sized orthogonal functional groups ${ }^{*}$}

\begin{abstract}
A reactive microcontact printing $(\mu \mathrm{CP})$ and dip-pen nanolithography (DPN) approach is described to create covalently immobilized nanoparticle and supramolecular host patterns. Amine functionalized $\beta$-cyclodextrin $\left(\beta \mathrm{CD}-\mathrm{NH}_{2}\right)$ and quantum dots $\left(\mathrm{QD}-\mathrm{NH}_{2}\right)$ have been printed and written onto $N, N$-carbonyldiimidazole-activated amino monolayer (IM monolayer)-functionalized glass slides, via the covalent formation of urethane bonds. The quantum dots patterns were directly imaged by exploiting their intrinsic fluorescence. To confirm the covalent formation of $\beta C D-\mathrm{NH}_{2}$ patterns, adamantyl-labeled fluorophores and ferrocenyl-labeled fluorescent proteins were used to image the obtained patterns. Additionally, orthogonal microcontact printing of $\beta-\mathrm{CD}$ and QD-NH $\mathrm{NH}_{2}$ patterns is succesfully demonstrated, furnishing a viable chemical strategy for the generation of patterns of different functional groups through reactive DPN for selective immobilization of multiple desired molecules with complementary functionalities.
\end{abstract}

${ }^{*}$ Manuscript in preparation 


\subsection{Introduction}

Introducing reactive functional units on monolayers offers the opportunity of anchoring desired molecules onto solid support surfaces via the formation of covalent bonds and therefore attracts enormous attention. By combining lithography techniques with reactive monolayers, patterns of desired ligand molecules at micro- or nanometer scale can be obtained,with potential applications in different fields. ${ }^{1-6}$ These processes may include single or multiple steps. The Whitesides group has utilized microcontact printing $(\mu \mathrm{CP})$ to respectively generate poly(ethylene-imine) patterns on carboxylic anhydride-terminated self-assembled monolayers (SAMs), ${ }^{7}$ and amino derivatives of biotin and benzenesulfonamide patterns on pentafluorophenylesters-terminated SAMs, ${ }^{8}$ via formation of amide bonds. We have also demonstrated patterning through microcontact printing amines on aldehyde-terminated SAMs via imine formation, ${ }^{9}$ and acetylene-containing molecules on azide-terminated SAMs via triazole formation. ${ }^{10,11}$ Several results have been reported where dip-pen nanolithography (DPN) is used to generate patterns on reactive monolayers. Mirkin's group demonstrated the Michael addition reaction of thiols and acrylamide moieties for covalently immobilizing oligonucleotides. ${ }^{12}$ Collier and co-workers also used the Michael addition reaction between thiols and maleimides for the generation of biotin patterns. ${ }^{13}$ In addition, the Diels-Alder reaction, ${ }^{14}$ borohydride reduction, ${ }^{15}$ triazole reaction, ${ }^{16,17}$ and amide-coupling reactions using $N$-hydroxysuccinimidyl ester $^{18,} 19$ and carboxylic anhydride ${ }^{20}$ are representative covalent organic reactions that have been applied with DPN.

$N, N$-carbonyldiimidazole (CDI)-activated amino monolayers (IM monolayers) developed in our group have been demonstrated by $\mu \mathrm{CP}$ to react with various functional groups including amino-, carboxyl- and alcohol groups, via the formation of urethane, amide and carbamate covalent bonds respectively. ${ }^{21}$ The rapid and efficient covalent reaction between amines and imidazolide groups is thought to be an advantage for efficient reactive DPN experiments. These IM monolayers can be used as platforms to immobilize a variety of functional molecules and $\beta$-cyclodextrin $(\beta C D)$ is one good candidate. $\beta C D$ is a water soluble cyclic oligosaccharide consisting of seven glucose units linked through $\alpha-1,4$ bonds to form a toroidal structure. ${ }^{22}$ The interior of the $\beta C D$ toroid is relatively hydrophobic. This specific geometrical shape and chemical anisotropy of $\beta C D$ molecules enable hosting of hydrophobic guest molecules within the toroid in aqueous media based on hydrophobic interactions. ${ }^{23}$ We have developed a platform, the so-called molecular printboard, that consists of $\beta$-cyclodextrin-terminated monolayers $(\beta C D$ 
monolayers) on different surfaces e.g. gold and glass substrates. ${ }^{24}$ A wide range of guest molecules or macromolecules containing suitable guest moieties, e.g. adamantyl moiety-labeled fluorescent molecules ${ }^{25}$ and proteins ${ }^{26}$ or ferrocene-labeled dendrimers ${ }^{27,28}$ have been placed onto the molecular printboard. $\beta$-cyclodextrin $(\beta C D)$ patterns on silicon oxide substrates have been fabricated by using nanoimprint lithography (NIL). ${ }^{29}$ The disadvantage of this technique is that it is difficult to introduce a second functional group onto reactive SAMs. However, by using $\mu \mathrm{CP}$ or DPN, the orthogonal printing or writing of molecules can be achieved relatively easily.

In this chapter, we first use $\mu \mathrm{CP}$ to print amine containing $\beta \mathrm{CD}$ and quantum dots (QDs- $\mathrm{NH}_{2}$ ) onto IM monolayer-functionalized glass slides, because of the simplicity and high-throughput production of $\mu \mathrm{CP}$. Fluorophores equipped with adamantyl moieties were used to confirm the formation of $\beta C D$ patterns. Subsequently, the atomic force fluorescence microscope (AFFM) was used to perform DPN accompanied by direct visualization of the results by atomic force microscopy (AFM) and confocal fluorescence microscopy. The experimental procedures are illustrated in Scheme 5.1. This is the first demonstration of using DPN to covalently anchor molecules onto IM monolayers. Additionally, the orthogonal printing of $\beta \mathrm{CD}$ and $\mathrm{QD}-\mathrm{NH}_{2}$ on IM monolayers is also demonstrated, setting the first step towards submicrometer patterns of orthogonal functional groups, including supramolecular cyclodextrin patterns. 


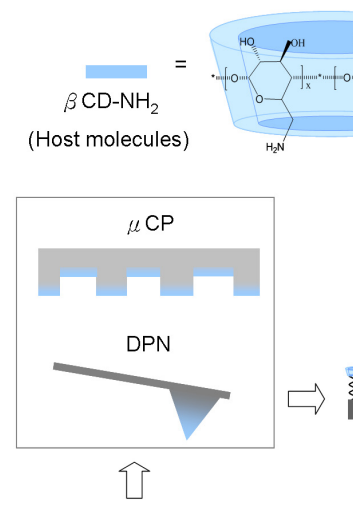

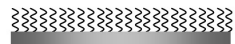

IM SAM
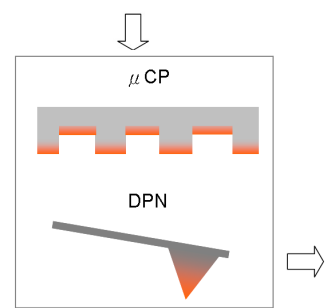

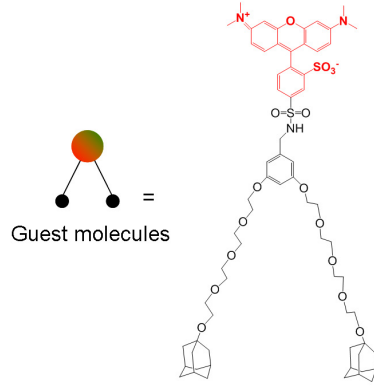

Lissamine-Ad

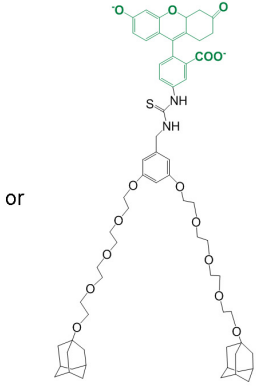

Fluorescein- $\mathrm{Ad}_{2}$

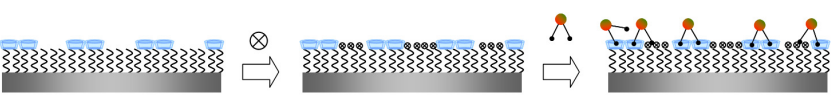

$\otimes$

Blocking molecules

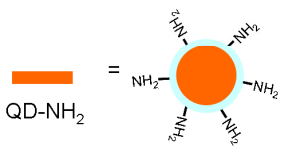

33333333333333333333333333333

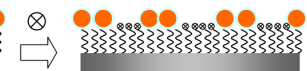

Scheme 5.1. Illustration of fabricating $\beta$-cyclodextrin $(\beta C D)$ and quantum dot $(Q D)$ patterns onto N,N-carbonyldiimidazole (CDI)-activated amino monolayers (IM monolayers) on glass substrates, with microcontact printing $(\mu C P)$ and dip-pen nanolithography (DPN). Lissamine and fluorescein-linked with adamantyl units were used to confirm formation of $\beta C D$ patterns.

\subsection{Results and Discussion}

$N, N$-carbonyldiimidazole (CDI)-activated amino monolayers utilized for $\mu \mathrm{CP}$ and DPN experiments were prepared by following the procedures reported by Hsu et al. and its synthesis process is depicted in Scheme 5.2 (see Experimental section 5.4). ${ }^{21} \mathrm{~A}$ two-step procedure was followed. Amino-terminated (TPEDA) monolayers were first formed by silylation of the cleaned glass substrates with ( $N$-[3-(trimethoxysilyl)propyl]ethylenediamine). Subsequently the TPEDA monolayers were converted into imidazolide 
monolayers (IM monolayers) by reacting CDI with the amino groups. By measuring the advancing water contact angles $\left(\theta_{\mathrm{a}}\right)$ of monolayers, a clear change in the polarity between TPEDA monolayers $\left(\theta_{\mathrm{a}}: \sim 64^{\circ}\right)$ and IM monolayers $\left(\theta_{\mathrm{a}}: \sim 55^{\circ}\right)$ was observed, which corresponds to reported values in the literature. ${ }^{21}$

\subsubsection{Fabrication of patterns with microcontact printing $(\mu \mathrm{CP})$}

\subsubsection{Fabricating $\beta$-cyclodextrin ( $\beta C D)$ patterns}

Per-7-amino- $\beta$-cyclodextrin $\left(\beta C D-\mathrm{NH}_{2}\right)$ (Scheme 5.1) was used as ink molecule. Non-oxidized poly(dimethylsiloxane) (PDMS) stamps with $10 \mu \mathrm{m}$ line features and a spacing of $5 \mu \mathrm{m}$, inked with $\beta \mathrm{CD}-\mathrm{NH}_{2}$, were brought into conformal contact with IM monolayer-functionalized glass slides for 5 minutes. After peeling off the stamps, the microcontact printed substrates were vigorously rinsed with MilliQ water to remove excess physisorbed $\beta \mathrm{CD}-\mathrm{NH}_{2}$ and then incubated into $0.1 \mathrm{M}$ ethanolamine aqueous solution for 30 minutes to passivate the non-reacted imidazolide groups. These substrates were rinsed with MilliQ water and then dried with nitrogen before examining with AFM. For measurements of the topography of the surfaces, the AFM was operated in contact mode. The height image and its average profile are shown in Figure 5.1a and 5.1b, respectively. The height of the pattern is $\sim 0.7 \mathrm{~nm}$ which indicates a single layer of $\beta C D-\mathrm{NH}_{2}$ lying flat on the surface. ${ }^{30}$ To confirm the reactivity of the $\beta C D-\mathrm{NH}_{2}$ patterns and their ability to exhibit host-guest interactions, lissamine equipped with two adamantyl

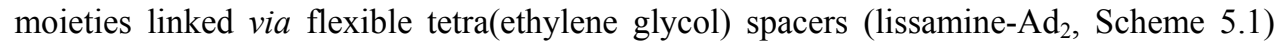
were used for fluorescent signaling. The fluorescence image and its average fluorescence intensity profile (Figure 5.1c and 5.1d) show that the lissamine- $\mathrm{Ad}_{2}$ area is consistent with the expected feature size of the microcontact printed $\beta C D$ region, indicating the quick immobilization of $\beta \mathrm{CD}-\mathrm{NH}_{2}$ onto IM monolayers since the microcontact printing time is only 5 minutes. 
a)

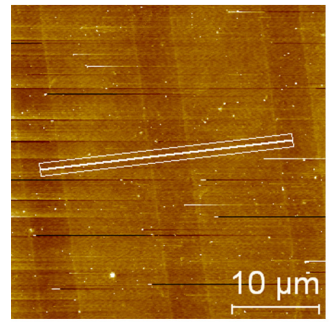

b)

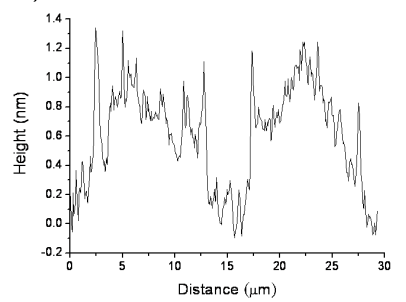

c)

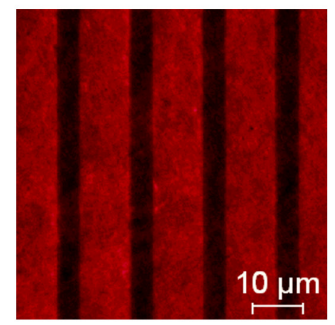

d)

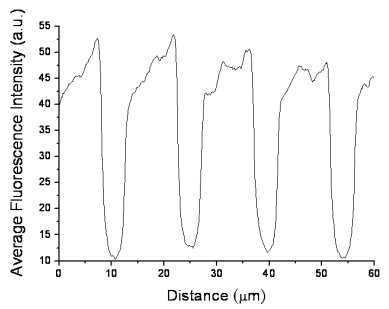

Figure 5.1. (a) AFM contact mode height image of $\beta C D-\mathrm{NH}_{2}$ ( $\mu$ CP patterns) covalently bound to IM monolayers. (b) Average height profile of the area defined by the white lines in (a). (c) Fluorescence images of lissamine-Ad $d_{2}$ adhered to $\beta C D-\mathrm{NH}_{2}$ via host-guest interaction. (d) Average fluorescence intensity profile of (c). Exposure time of the camera was $400 \mathrm{~ms}$.

Fabricated $\beta C D$ patterns can also be used as an indirect strategy to immobilize guest moieties-labeled proteins with controlled orientation, which provides potential applications described in the introductions of Chapter 2 and Chapter 4. Non-oxidized $\beta C D-\mathrm{NH}_{2}$ inked PDMS stamps with $5 \mu \mathrm{m}$ line features and a spacing of $3 \mu \mathrm{m}$ were brought into conformal contact with IM monolayers-functionalized glass slides for 20 minutes. The remaining active imidazolide groups were blocked by incubating the substrate with aqueous ethanolamine solution for 30 minutes. Yellow fluorescent protein labeled with a ferrocenyl moiety (Fc-YFP) was used to adhere to printed $\beta C D-\mathrm{NH}_{2}$ patterns. The longer microcontact printing time (20 minutes) was used in order to have more $\beta C D-\mathrm{NH}_{2}$ adhered to IM-monolayers, in accordance with previous reports that the maximum fluorescence intensity was obtained after printing 4-(2-aminoethylamino)-7( $N, N$-dimethylsulfamoyl)benzofurazan (ADF) with IM monolayers for 20 minutes. $^{21}$ The fluorescent image of Fc-YFP (Figure 5.2a) shows the protein patterns with the expected feature size. 
a)

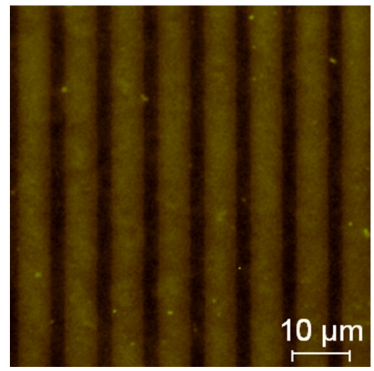

b)

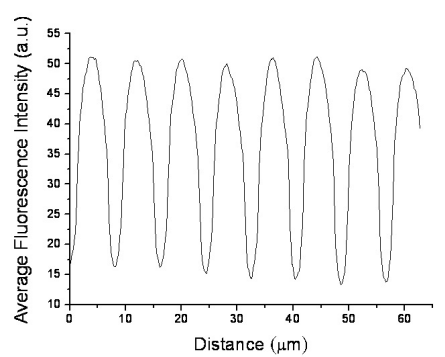

Figure 5.2. (a) Fluorescence images of Fc-YFP adhered to $\beta C D-\mathrm{NH}_{2}$ patterns via host-guest interaction. (b) The average fluorescence intensity profile of (a). The exposure time of the camera to obtain the image is $2 \mathrm{~s}$.

\subsubsection{Fabricating quantum dot patterns}

Amine-containing quantum dots (QDs- $\mathrm{NH}_{2}$, eBioscience Inc.) (Scheme 5.1) were also used as ink. The emission wavelength of QDs- $\mathrm{NH}_{2}$ is $605 \mathrm{~nm}$. Non-oxidized poly(dimethylsiloxane) (PDMS) stamps with $5 \mu \mathrm{m}$ line features and a spacing of $3 \mu \mathrm{m}$, inked with QDs- $\mathrm{NH}_{2}$ were brought into conformal contact with IM monolayers-functionalized glass slides for 20 minutes. After removing the stamps, the microcontact printed substrates were vigorously rinsed with Milli-Q water and then examined with a fluorescence microscope. Figure 5.3 shows the successful transfer of QDs- $\mathrm{NH}_{2}$ from the PDMS stamp to IM monolayers.

a)

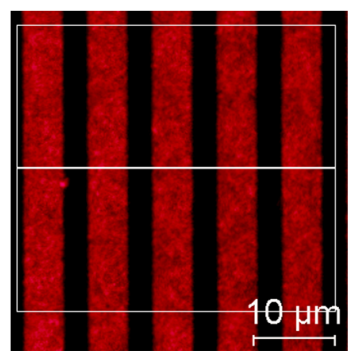

b)

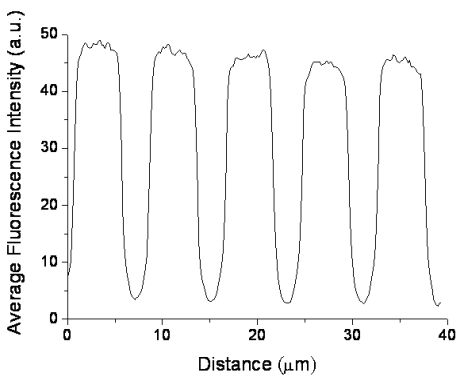

Figure 5.3. a) Fluorescence image of $Q D-\mathrm{NH}_{2}$ patterns via covalent bonding between amine and imidazolide groups. b) Average fluorescence intensity profile of the area inside the rectangle indicated in (a). Exposure time was $100 \mathrm{~ms}$. 


\subsubsection{Fabricating $\beta$-cyclodextrin and quantum dot patterns together}

The remaining active imidazolide groups after patterning QDs- $\mathrm{NH}_{2}$ on IM monolayers can subsequently react with $\beta C D-\mathrm{NH}_{2}$. To have clear fluorescent patterns a non-oxidized PDMS stamp with $10 \mu \mathrm{m}$ diameter circular features separated with a distance of $5 \mu \mathrm{m}$ was used. After 20 minutes of printing QDs- $\mathrm{NH}_{2}$, the patterned substrate was rinsed with water, dried with $\mathrm{N}_{2}$ and then immediately incubated with $0.5 \mathrm{mM}$ $\beta C D-\mathrm{NH}_{2}$ aqueous solution for 30 minutes. To distinguish the $\beta C D-\mathrm{NH}_{2}$ region from QDs- $\mathrm{NH}_{2}$, fluorescein equipped with two adamantyl moieties (fluorescein- $\mathrm{Ad}_{2}$, Scheme 5.1) was used to visualize the $\beta C D-\mathrm{NH}_{2}$ area. (Figure 5.4a-c) This experimental procedure was also performed in the opposite sequence, i.e. printing $\beta C D-\mathrm{NH}_{2}$ first and then functionalizing the unprinted region with QDs- $\mathrm{NH}_{2}$ (Figure $5.4 \mathrm{~d}$-e). Fluorescence images of QD-NH $\mathrm{NH}_{2}$ (Figure 5.4a and 5.4d) and fluorescein- $\mathrm{Ad}_{2}$ (Figure 5.4b and 5.4e) patterns were obtained separately using suitable filter cubes.

Figure 5.4 demonstrates the successful surface modification of non-printed areas of the IM monolayers and indicates the potential of orthogonal microcontact printing of different inks. To explore this further, a PDMS stamp with $10 \mu \mathrm{m}$ line features and a spacing of $5 \mu \mathrm{m}$ inked with QDs- $\mathrm{NH}_{2}$ was first brought into conformal contact with the IM monolayer-functionalized surface for 20 minutes. Another stamp inked with $\beta C D-\mathrm{NH}_{2}$ was subsequently brought into contact with the same substrate in the orthogonal direction for 20 minutes. After blocking the remaining active imidazolide groups with ethanolamine, this substrate was incubated into fluorophore solution to fill printed $\beta C D-\mathrm{NH}_{2}$ area with fluorescein- $\mathrm{Ad}_{2}$. Clear fluorescence line patterns of QDs- $\mathrm{NH}_{2}$ (Figure 5.5a) and well-defined areas of fluorescein- $\mathrm{Ad}_{2}$ (Figure 5.5b) were obtained, demonstrating successful orthogonal microcontact printing of the two inks. 
a)

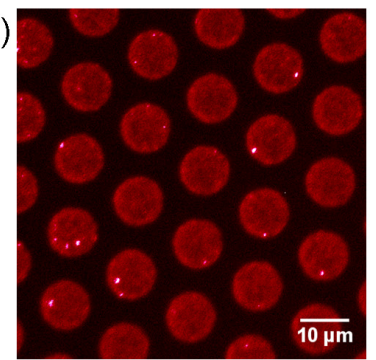

d)

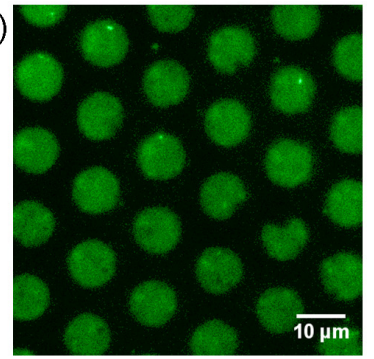

b)

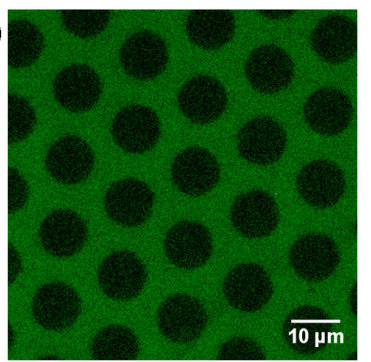

e)

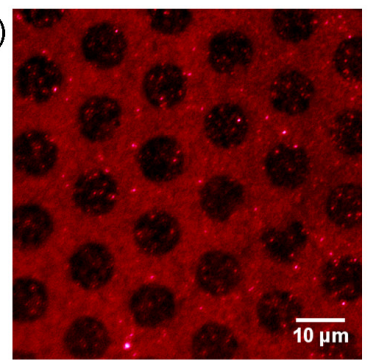

c)

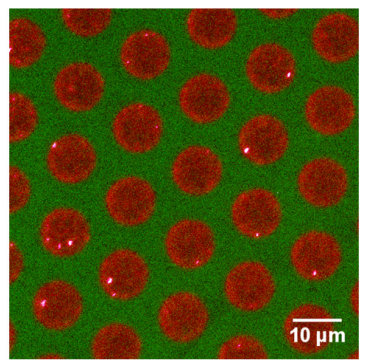

f)

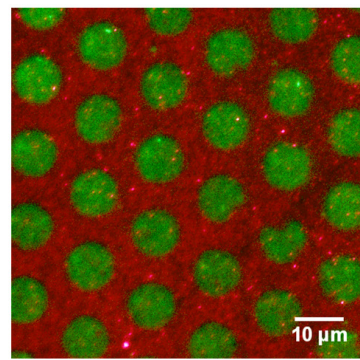

Figure 5.4. Fluorescence images (exposure time $100 \mathrm{~ms}$ ). Top, (a) microcontact printed $Q D-\mathrm{NH}_{2}$ patterns, (b) unprinted $Q D-\mathrm{NH}_{2}$ regions reacted with $\beta C D-\mathrm{NH}_{2}$ and then filled with fluorescein-Ad, and (c) the added result of (a) and (b). Bottom, (d) microcontact printed $\beta \mathrm{CD}-\mathrm{NH}_{2}$ patterns consecutively filled with fluorescein-Ad, (e) the unprinted $\beta C D-\mathrm{NH}_{2}$ region shown in (d) functionalized with $\mathrm{QD}-\mathrm{NH}_{2}$, and (f) the added result of (d) and $(e)$.

a)

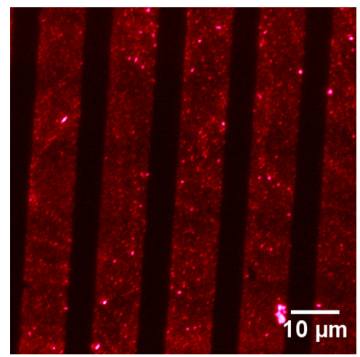

b)

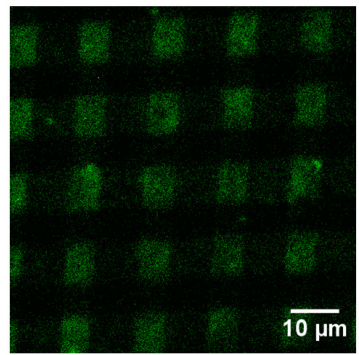

c)

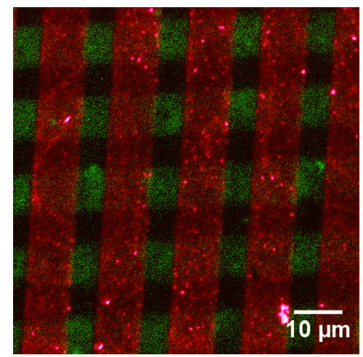

Figure 5.5. Fluorescence images of (a) $Q D-\mathrm{NH}_{2}$ patterns via the covalent bonding between amine and imidazolide groups, (b) Fluorescein-Ad 2 reacted with microcontact printed region of $\beta C D-\mathrm{NH}_{2}$. (c) The added result of (a) and (b). The exposure time of (a) and (b) is 100ms. 


\subsubsection{Fabrication of patterns with dip-pen nanolithography (DPN)}

\subsubsection{Fabricating quantum dot patterns}

Here, QDs- $\mathrm{NH}_{2}$ were used as ink first for DPN experiments because their intrinsic fluorescence enables immediate visualization of the patterning results in situ on the atomic force fluorescence microscope (AFFM). To prove the reactive covalent bond formation of urethane, commercial $\mathrm{Si}_{3} \mathrm{~N}_{4}$ AFM tips incubated in a $2 \mu \mathrm{M}$ QDs- $\mathrm{NH}_{2}$ solution were used to generate quantum dot patterns on both IM monolayer-functionalized and cleaned bare glass substrates. The QDs- $\mathrm{NH}_{2}$-coated tip was brought into contact with an IM monolayer-functionalized surface to continuously generate 5 single vertical lines of $\sim 7.2$ $\mu \mathrm{m}$ in length without re-inking the tip. The 5 lines (from left to right) were written with a scan rate of $\sim 28 \mu \mathrm{m} / \mathrm{s}$ for $1,2,3,4$ and 5 minutes at $\sim 40 \%$ relative humidity (RH). The fluorescence image of the 5-lines pattern was acquired by the confocal microscope of the AFFM immediately after writing (Figure 5.6a) and the average fluorescence intensity profile (Figure 5.6c) was extracted, which indicated the successful transfer of QDs- $\mathrm{NH}_{2}$ from the tip to the substrate. The width of the quantum dot line is $\sim 1 \mu \mathrm{m}$. The written area was subsequently rinsed with HPLC water carefully and imaged (Figure 5.6b). The average fluorescence intensity of each line acquired after rinsing decreases showing some physisorbed QDs- $\mathrm{NH}_{2}$ were removed (Figure 5.6d). Nevertheless, the 5-lines quantum dot pattern still can be clearly observed in Figures 5.6b and 5.6d confirming the formation of irreversible covalent bonds through reactive DPN.

A control DPN experiment was performed by bringing a QDs- $\mathrm{NH}_{2}$-coated tip into contact with a cleaned bare glass substrate to write $\mathrm{a} \sim 1.5 \mu \mathrm{m}$ wide square in one move with a writing speed of $\sim 7 \mu \mathrm{m} / \mathrm{s}$. A bright square pattern was obtained immediately after writing showing the deposition of QDs- $\mathrm{NH}_{2}$ (Figure 5.6e). However, the QD- $\mathrm{NH}_{2}$ pattern did not stay on the substrate after rinsing with HPLC water, which indicated the lack of strong interactions between quantum dots and substrate (Figure 5.6f). 


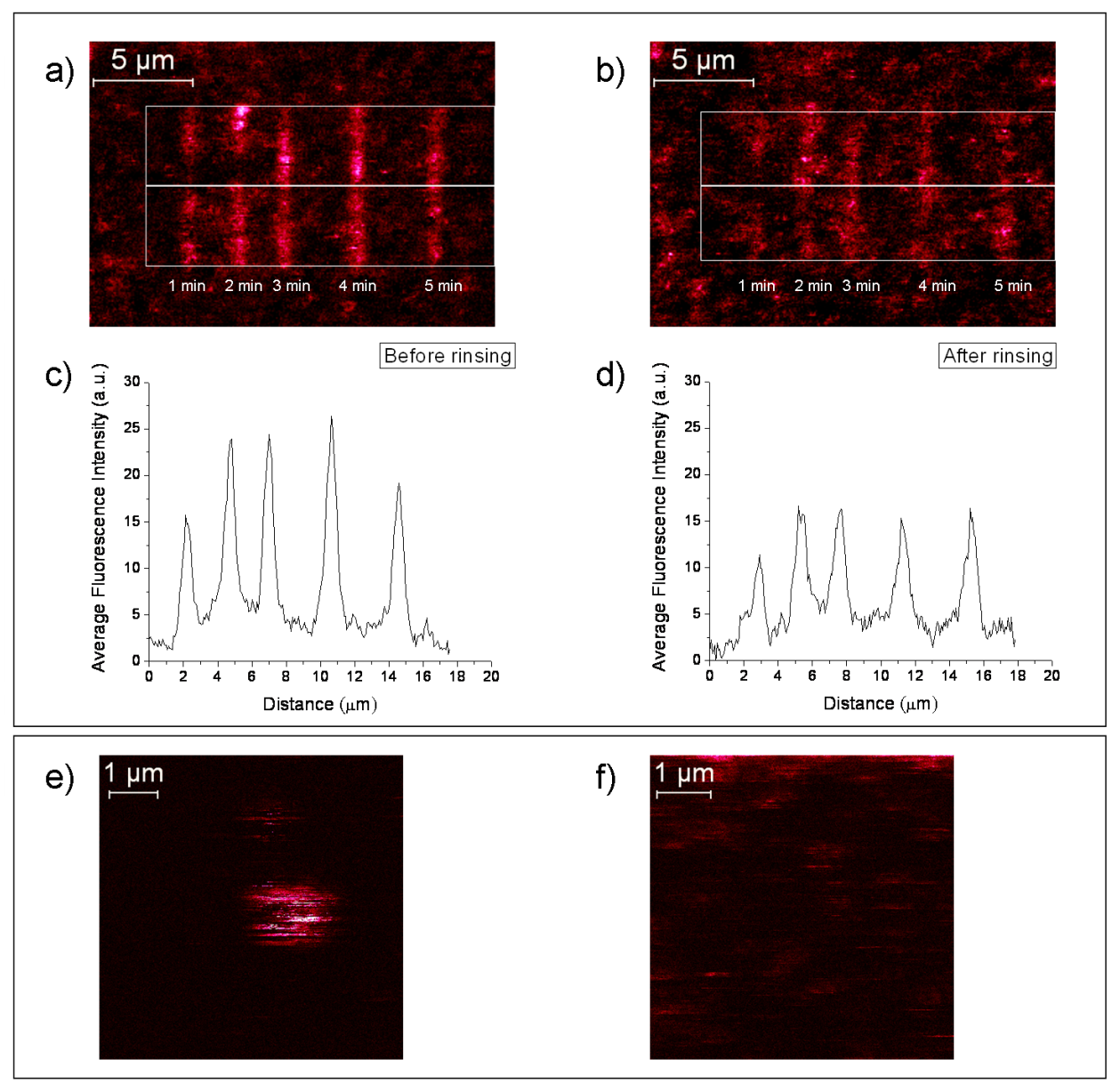

Figure 5.6. Fluorescence image of five vertical lines ( $7.2 \mu \mathrm{m}$ in length) were successively generated from left to right by using an AFM tip inked with QDs-NH $\mathrm{H}_{2}$ using a scanning speed of $28 \mu \mathrm{m} / \mathrm{s}$ for varied times (a) before and (b) after rinsing with HPLC water. The scanning time of each line is listed on the images. (c) and (d) The average fluorescence intensity profile of the area inside the rectangle indicated in (a) and (b), respectively. Fluorescence images of a square $Q D-N_{2}$ pattern $\left(\sim 1.5 \times 1.5 \mu m^{2}\right)$ written onto a cleaned bare glass substrate (e) before and (f) after rinsing with HPLC water. The exposure time of all images is $0.25 \mathrm{~ms}$ per pixel. 


\subsubsection{Fabricating $\beta$-cyclodextrin ( $\beta C D)$ patterns}

Commercial $\mathrm{Si}_{3} \mathrm{~N}_{4}$ AFM tips incubated in filtered saturated $\beta C D-\mathrm{NH}_{2}$ aqueous solution overnight were used to fabricate $\beta C D$ patterns which were confirmed by complexing with fluorescein- $\mathrm{Ad}_{2}$ for fluorescent signaling via host-guest interaction. Using the AFFM at $\sim 40 \%$ relative humidity $(\mathrm{RH})$, the $\beta \mathrm{CD}-\mathrm{NH}_{2}$-coated tip was brought into contact with an IM monolayer-functionalized and a cleaned bare glass substrate to scan $\mathrm{a} \sim 3.6 \mu \mathrm{m}$ wide square once with a writing speed of $\sim 1.4 \mu \mathrm{m} / \mathrm{s}$. After DPN, the topographic information of the $\beta C D$ patterns was revealed by AFM imaging. To avoid contaminations, a new $\mathrm{Si}_{3} \mathrm{~N}_{4}$ AFM tip was brought to the original position in the DPN experiments, directly above the central focus of the objective lens. The topography measurements were performed in tapping mode and are shown in Figure 5.7a and 5.7b indicating the transfer of material from the tip to the substrate. The corresponding average height profiles (Figures 5.7c and 5.7d) show that multilayers of $\beta C D-\mathrm{NH}_{2}$ adhered to both substrates.

After passivation of the remaining imidazolide groups on the IM monolayer with 0.1 $\mathrm{M}$ ethanolamine aqueous solution, fluorescein- $\mathrm{Ad}_{2}$ solution was placed onto the written area on both bare and IM monolayer-functionalized glass substrates for confirming the immobilization of $\mathrm{CD}$ by reactive DPN. After removing fluorescein- $\mathrm{Ad}_{2}$ solution and rinsing the written areas with saturated $\beta$-cyclodextrin $(\beta C D)$ aqueous solution and HPLC water, the written areas were subsequently imaged with AFFM (Figures 5.8a and 5.8b). It can be clearly observed that the fluorescent square pattern is only observed on the IM monolayer-functionalized glass substrate, again indicating the formation of covalent bonds that have been successfully obtained using DPN. The average fluorescence intensity profile of the fluorescein- $\mathrm{Ad}_{2}$ pattern is shown in Figure 5.8c. 
a)
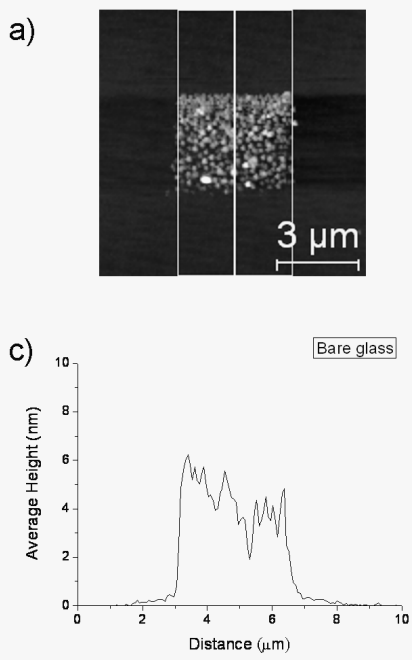

d)

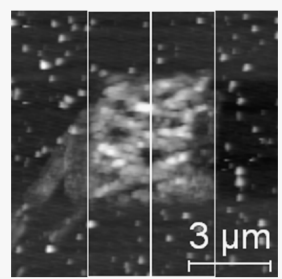

b)

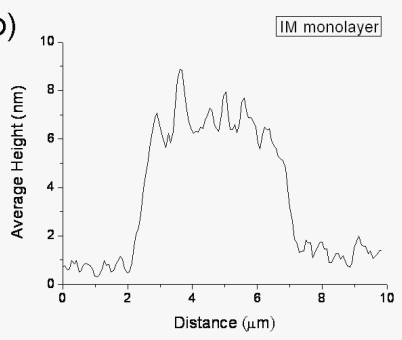

Figure 5.7. (a) AFM tapping mode height image of $\beta C D$ patterns fabricated by depositing $\beta C D-\mathrm{NH}_{2}$ with dip-pen nanolithography onto (a) a cleaned bare glass substrate and (b) IM monolayer-functionalized glass substrate. Some white spots shown outside of the square area in (a) result from the N,N-carbonyldiimidazole (CDI) adsorbed onto the surface while preparing IM monolayer. (c) and (d) Average height profile of the area inside the rectangle indicated in (a) and (b), respectively.

a)

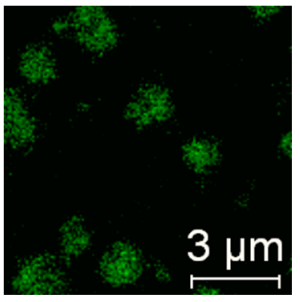

b)

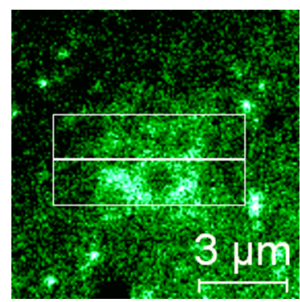

c)

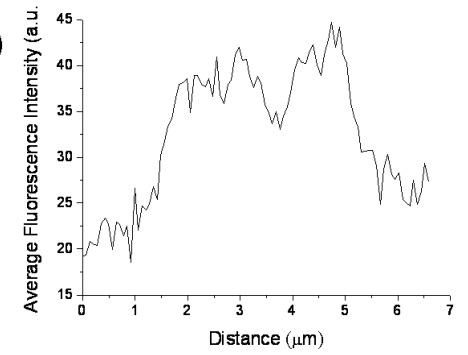

Figure 5.8. Fluorescence image of $\beta C D-\mathrm{NH}_{2}$ pattern generated by dip-pen nanolithography on (a) a cleaned bare glass substrate and (b) a IM monolayer-functionalized glass substrate after reacting with fluorescein- $\mathrm{Ad}_{2}$ via host-guest interaction. The exposure time of the fluorescence image obtained by AFFM is $0.25 \mathrm{~ms}$ per pixel. The excitation laser power for obtaining figure (a) is 10 times higher than for figure (b). (c) The average fluorescence intensity profile of the area defined by the white lines shown in (b). 
In Figure 5.8, the $\beta C D-\mathrm{NH}_{2}$ pattern was generated with a writing speed of $\sim 1.4 \mu \mathrm{m} / \mathrm{s}$. To demonstrate that the patterns can be fabricated within a shorter time, another $\beta C D-\mathrm{NH}_{2}$-coated tip was brought into contact with an IM monolayer-functionalized surface to continuously generate 2 squares of $\sim 3.6 \mu \mathrm{m}$ in width without re-inking the tip. The 2 squares (from right to left) were written once with a scan rate of $\sim 28$ and $\sim 14 \mu \mathrm{m} / \mathrm{s}$ respectively, at $\sim 40 \%$ relative humidity $(\mathrm{RH})$,. After passivating the un-patterned area and filling the $\mathrm{CD}$ area with fluorescein- $\mathrm{Ad}_{2}$, the fluorescence image and its average fluorescence intensity profile (Figure $5.9 \mathrm{a}$ and $5.9 \mathrm{~b}$ ) show that fluorescein- $\mathrm{Ad}_{2}$ patterns are consistent with the expected feature sizes of the written $\beta C D-\mathrm{NH}_{2}$ region. This result indicates that $\sim 3.6 \mu \mathrm{m}$ wide patterns of covalently immobilized $\beta \mathrm{CD}$ can be fabricated within a minute. Comparing with the fluorescent images obtained by $\mu \mathrm{CP}$ (Figures 5.1 and 5.2), much higher background signals are obtained while performing DPN experiments (Figures 5.8b and 5.9a). This difference may be attributed to the difficulty of rinsing written areas right above the objective lens without moving the substrate.

a)

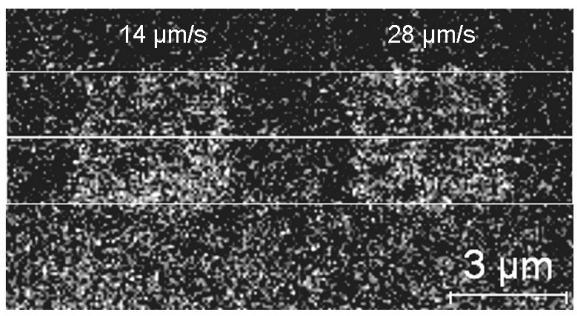

b)

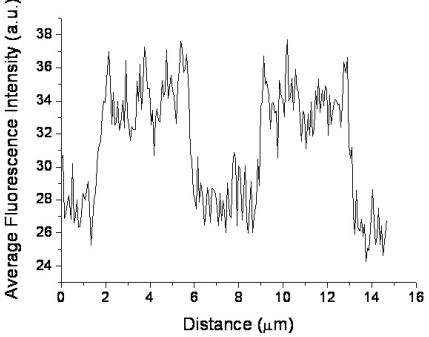

Figure 5.9. (a)Fluorescence image of $2 \beta C D-\mathrm{NH}_{2}$ square pattern generated by dip-pen nanolithography with a scanning speed of $\sim 28$ and $\sim 14 \mu \mathrm{m} / \mathrm{s}$ (from right to left) respectively on a IM monolayer-functionalized glass substrate after reacting with fluorescein-Ad $\mathrm{d}_{2}$ via host-guest interaction. The exposure time of the fluorescence image obtained by AFFM is $0.25 \mathrm{~ms}$ per pixel. (b) The average fluorescence intensity profile of the area defined by the white lines shown in (a). 
Although the DPN results are still clearly not optimized, section 5.2.2 demonstrates preliminary DPN results showing that the covalent reaction between imidazolide groups and amine groups can be obtained after DPN. Since DPN allows one to create patterns with a feature size of tens of nanometers, we expect to be able to achieve sub-micrometer patterns of $\beta C D-\mathrm{NH}_{2}$ and $\mathrm{QDs}-\mathrm{NH}_{2}$ in the near future. Additionally, it has been demonstrated in Section 5.2.1.3 that $\beta \mathrm{CD}-\mathrm{NH}_{2}$ and $\mathrm{QDs}-\mathrm{NH}_{2}$ patterns can be fabricated together using orthogonal microcontact printing. Therefore, we believe that this chemical approach can be used with DPN to generate different types of amino-containing functional groups onto IM monolayer-functionalized surfaces for selectively anchoring desired molecules.

\subsection{Conclusions}

In summary, a covalent reaction approach has been developed for immobilizing amine-containing $\beta$-cyclodextrin and quantum dots $\left(\beta C D-\mathrm{NH}_{2}\right.$ and $\left.\mathrm{QD}-\mathrm{NH}_{2}\right)$ at micrometer scale onto $N, N$-carbonyldiimidazole-activated amino monolayer (IM monolayer)- functionalized glass slides via the covalent formation of urethane bonds by microcontact printing $(\mu \mathrm{CP})$ and dip-pen nanolithography (DPN). Fluorophores equipped with adamantyl moieties (fluorecein- $\mathrm{Ad}_{2}$ and lissamine- $\mathrm{Ad}_{2}$ ) and yellow fluorescent proteins labeled with ferrocenyl moieties (Fc-YFP) were used for fluorescence microscopy confirmation of the formation of covalently immobilized $\beta C D-\mathrm{NH}_{2}$ patterns. The orthogonal microcontact printing of $\beta C D-\mathrm{NH}_{2}$ and $\mathrm{QD}-\mathrm{NH}_{2}$ has also successfully been demonstrated. Therefore this chemical strategy provides the possibility of writing different types of amino-containing functional groups with DPN onto IM monolayer-functionalized surfaces at sub-micrometer scales for selective anchoring of desired molecules in the near future, e.g. as an indirect DPN approach to generate multiple protein patterns. 


\subsection{Experimental}

\section{General procedures}

All glassware used to prepare the monolayers was cleaned by sonicating for 60 minutes in a $2 \% \mathrm{v} / \mathrm{v}$ Hellmanex II solution in high purity water (Milli-Q, $18.2 \mathrm{M} \Omega \mathrm{cm}$ ), and then rinsed thoroughly with Milli-Q water, and dried in an ambient environment. Microscope glass slide substrates were cleaned in piranha solution for 20 minutes, (concentrated $\mathrm{H}_{2} \mathrm{SO}_{4}$ and $33 \%$ aqueous $\mathrm{H}_{2} \mathrm{O}_{2}$ in a $3: 1$ ratio). [Warning: Piranha solution should be handled with caution: it has been reported to detonate unexpectedly.] The glass slides were then rinsed several times with Milli-Q water, and dried gently under a nitrogen stream before forming the monolayer.

\section{Imidazolide monolayer preparation.}

The freshly cleaned microscope glass slide substrates were placed in a desiccator under vacuum overnight for the gas-phase evaporation of $N$-[3-(trimethoxysilyl)propyl]ethylenediamine to form amino-terminated monolayer (TPEDA monolayer). After the TPEDA monolayer-functionalized substrates were taken out from the desiccator, they were rinsed carefully with ethanol and dichloromethane to remove physisorbed materials and then dried under a stream of $\mathrm{N}_{2}$. In order to obtain more stable TPEDA monolayer, the substrates were placed in an oven at $110{ }^{\circ} \mathrm{C}$ for 3 hours before reacting with $N, N$-carbonyldiimidazole (CDI). The TPEDA monolayer-functionalized substrates were then incubated with saturated CDI solution in freshly distilled Tetrahydrofuran (THF) under Argon overnight. ${ }^{21}$ After the formation of imidazolide monolayer, the substrates were rinsed with THF, followed by nitrogen drying. All samples were freshly prepared and used immediately after drying.

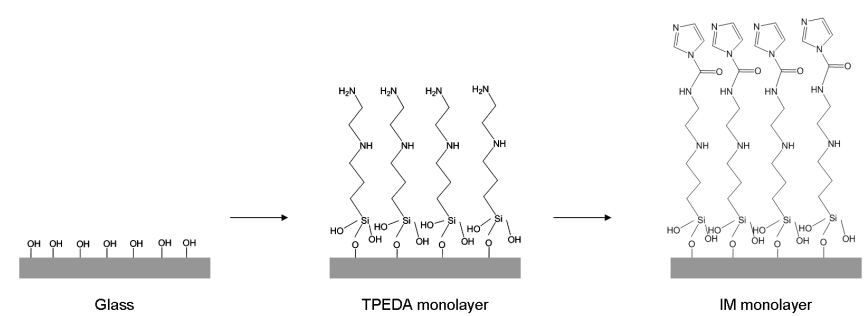

Scheme 5.2. Illustration of the preparation procedure for an imidazolide-terminated monolayer (IM monolayers) on glass.

\section{Microcontact printing $(\mu \mathrm{CP})$.}

The preparation procedure of PDMS stamps were described in Chapter 3. The non-oxidized PDMS stamps were incubated in $0.5 \mathrm{mM}$ per-7-amino- $\beta$-cyclodextrin $\left(\beta \mathrm{CD}-\mathrm{NH}_{2}\right)$ aqueous solution or 500 $\mathrm{nM}$ amine-containing quantum dots (QDs- $\mathrm{NH}_{2}$, eFluor ${ }^{\mathrm{TM}} 605^{\mathrm{NC}}$ (Amine), eBioscience, Inc. USA) 
aqueous solution for at least 20 minutes and then dried with a nitrogen stream. The inked stamps were subsequently brought into contact with IM monolayers-functionalized glass slides for defined time without the use of external pressure. After removing the stamps, the printed substrates were rinsed vigorously with Milli-Q water and then dried. The $\beta C D-\mathrm{NH}_{2}$ printed substrates were immediately incubated into $0.1 \mathrm{M}$ ethanolamine aqueous solution for 30 minutes to block the remaining active imidazolide groups and then rinsed with MilliQ water to remove excess physisorbed materials before drying with $\mathrm{N}_{2}$. These printing procedures were also applied in the experiment of cross printing.

The $\beta \mathrm{CD}-\mathrm{NH}_{2}$ printed substrates were incubated into an $\sim 1 \mu \mathrm{M}$ aqueous solution of lissamine equipped with two adamantyl moieties linked via flexible tetra(ethylene glycol) spacers (lissamine- $\mathrm{Ad}_{2}$ ) for 10 minutes. After removing substrates from the fluorophore solution, they were rinsed vigorously with $1 \mathrm{mM} \beta$-cyclodextrin $(\beta C D)$ aqueous solution and Milli-Q water mixed with $1 \%(\mathrm{v} / \mathrm{v})$ ethanol to remove the excess physisorbed fluorophores and then dried. The $\beta \mathrm{CD}-\mathrm{NH}_{2}$ patterned substrates were also incubated into $\sim 1 \mu \mathrm{M}$ solution in PBS buffer $(\mathrm{pH} 7.4)$ of ferrocene derivative yellow fluorescent protein (Fc-YFP) for 30 minutes and then rinsed with $\mathrm{pH} 7.4$ PBS buffer and Milli-Q water to remove excess proteins and then dried.

The printed QDs- $\mathrm{NH}_{2}$ patterns were also incubated into $0.5 \mathrm{mM} \beta \mathrm{CD}-\mathrm{NH}_{2}$ aqueous solution for 30 minutes to backfill the unprinted region and then vigorously rinsed with Milli-Q water. These substrates were incubated into an $\sim 1 \mu \mathrm{M}$ aqueous solution of fluorescein equipped with two adamantyl moieties linked via flexible tetra(ethylene glycol) spacers (fluorescein- $\mathrm{Ad}_{2}$ ) for 10 minutes. After removing substrates from the fluorophore solution, they were rinsed with $1 \mathrm{mM} \beta \mathrm{CD}$ aqueous solution and Milli-Q water mixed with $1 \%(\mathrm{v} / \mathrm{v})$ ethanol and then dried. This experimental procedure was also performed when printing $\beta \mathrm{CD}-\mathrm{NH}_{2}$ and then backfilling with QDs- $\mathrm{NH}_{2}$.

\section{Acquisition of fluorescent images.}

All these pattered surfaces with $\mu \mathrm{CP}$ were imaged with a fluorescence microscope (TE2000-U, Nikon) equipped with a mercury lamp as light source and a digital camera (Nikon digital Sight DS-Qi1Mc). To obtain fluorescence images of QD- $\mathrm{NH}_{2}$ and lissamine- $\mathrm{Ad}_{2}$ patterns, a filter cube with green excitation light $(\lambda=510 / 40 \mathrm{~nm})$, red emission light $(\lambda=617 / 73 \mathrm{~nm})$ and dichroic $(\lambda=562$ $\mathrm{nm}$ ) was used. To obtain fluorescence images of Fc-YFP and fluorescein-Ad $\mathrm{A}_{2}$ patterns, a filter cube with blue excitation light $(\lambda=470 / 22 \mathrm{~nm})$, green emission light $(\lambda=520 / 35 \mathrm{~nm})$ and dichroic $(\lambda=495$ $\mathrm{nm})$ was used. The fluorescence images obtained were analyzed with the commercial software SPIP (Image Metrology, Version 4.4.3.0) and ImageJ (Version 1.40g).

\section{AFM imaging of microcontact printed patterns.}

AFM topography measurements in height were carried out with a custom-built atomic force fluorescence microscope (AFFM) in contact mode, with 512 × 512 pixels acquisitions. Commercial 
$\mathrm{Si}_{3} \mathrm{~N}_{4}$ AFM cantilevers (Veeco Probes) with nominal spring constant of $0.54 \mathrm{~N} / \mathrm{m}$ were used. The scan speed is 500 pixels per second.

\section{Dip pen nanolithography (DPN) and visualization with AFFM.}

AFFM was also used to perform dip-pen nanolithography and to observe the results of patterning in situ without having to change to another instrument. Commercial $\mathrm{Si}_{3} \mathrm{~N}_{4}$ AFM cantilevers (Veeco Probes) with nominal spring constant of $0.32 \mathrm{~N} / \mathrm{m}$ were used. Immediately before a DPN experiment, the cantilever was rinsed rigorously with ethanol and dried under a stream of $\mathrm{N}_{2}$. Cleaned cantilevers were then immersed into $2 \mu \mathrm{M}$ QD-NH 2 solution for at least 15 minutes or filtered saturated $\beta C D-\mathrm{NH}_{2}$ aqueous solution overnight and then dried with $\mathrm{N}_{2}$. The QDs- $\mathrm{NH}_{2}$ or $\beta C D-\mathrm{NH}_{2}$-coated cantilever was mounted on the AFM head and used to carry out DPN experiments on IM monolayers-functionalized or cleaned bare glass substrates in contact mode inside a custom-built chamber with a controlled humidity $(\sim 40 \%)$ at a temperature between 20 and $22{ }^{\circ} \mathrm{C}$.

After depositing QDs- $\mathrm{NH}_{2}$, the fluorescence image of the generated quantum dot pattern was obtained by exciting with the $488 \mathrm{~nm}$ line of an Argon-ion laser (163-D Laser System, Spectra Physics) and then recording the fluorescent signals with an avalanche photodiode (APD) (SPCM-AQR-14, Perkin Elmer Optoelectronics). The same area was imaged again after carefully rinsing with HPLC water to remove the physisorbed materials and then drying under a stream of $\mathrm{N}_{2}$ in situ to avoid displacement of the patterned area from the observation range of the objective.

Before complexing $\beta C D-\mathrm{NH}_{2}$ with fluorescein- $\mathrm{Ad}_{2}$ for fluorescent imaging, the topography of generated $\beta \mathrm{CD}-\mathrm{NH}_{2}$ square patterns on either IM monolayers-functionalized or cleaned bare glass substrates was characterized by AFM in tapping mode. The original ink-coated tip was replaced by a new $\mathrm{Si}_{3} \mathrm{~N}_{4}$ AFM tip (Veeco Probes) with nominal spring constant of $0.54 \mathrm{~N} / \mathrm{m}$ to prevent contamination of the patterned areas during subsequent imaging. The image was obtained with 256 x 256 pixels acquisitions and scanning speeds of 2000 pixels per second. Subsequently, the written $\beta C D-\mathrm{NH}_{2}$ pattern on the IM monolayer-functionalized substrate was incubated with $5 \mu$ of $0.1 \mathrm{M}$ ethanolamine aqueous solution for 30 minutes to block the remaining active imidazolide groups and then rinsed with a few drops of HPLC water before drying with $\mathrm{N}_{2} .5 \mu \mathrm{l}$ of $200 \mathrm{nM}$ fluorescein- $\mathrm{Ad}_{2}$ solution was placed onto the written area on both substrates for 5 minutes. To remove the excess fluorescein- $\mathrm{Ad}_{2}$ adsorbed onto the surface, $5 \mu$ of filtered saturated $\beta$-cyclodextrin $(\beta C D)$ aqueous solution was placed onto the written region for 5 minutes. After removing $\beta C D$ solution, the $\beta C D-\mathrm{NH}_{2}$ deposited areas were subsequently rinsed with few drops of HPLC water before drying with $\mathrm{N}_{2}$. The fluorescein- $\mathrm{Ad}_{2}$ patterns were also excited with the $488 \mathrm{~nm}$ line of an Argon-ion laser and the emission of the fluorescence was recorded using an APD. Both fluorescence and AFM topographic images were analyzed and prepared with the commercial software SPIP (Image Metrology, Version 4.4.3.0). 


\subsection{Acknowledgements}

I acknowledge Dr. Arántzazu González-Campo and Dr. Shu-Han Hsu for generously sharing their knowledge on preparing the IM monolayer and Dr. Pascal Jonkheijm for generously offering ferrocene derivative yellow fluorescent protein.

\subsection{References}

1. Smith, R. K.; Lewis, P. A.; Weiss, P. S. Patterning Self-Assembled Monolayers. Prog. Surf. Sci. 2004, 75, $1-68$.

2. Li, X. M.; Huskens, J.; Reinhoudt, D. N. Reactive Self-Assembled Monolayers on Flat and Nanoparticle Surfaces, and Their Application in Soft and Scanning Probe Lithographic Nanofabrication Technologies. J. Mater. Chem. 2004, 14, 2954-2971.

3. Arya, S. K.; Solanki, P. R.; Datta, M.; Malhotra, B. D. Recent Advances in Self-Assembled Monolayers Based Biomolecular Electronic Devices. Biosensors \& Bioelectronics 2009, 24, 2810-2817.

4. Gooding, J. J.; Mearns, F.; Yang, W. R.; Liu, J. Q. Self-Assembled Monolayers into the 21(St) Century: Recent Advances and Applications. Electroanalysis 2003, 15, 81-96.

5. Kramer, S.; Fuierer, R. R.; Gorman, C. B. Scanning Probe Lithography Using Self-Assembled Monolayers. Chem. Rev. 2003, 103, 4367-4418.

6. Rosa, L. G.; Liang, J. Atomic Force Microscope Nanolithography: Dip-Pen, Nanoshaving, Nanografting, Tapping Mode, Electrochemical and Thermal Nanolithography. Journal of Physics-Condensed Matter 2009, 21, 483001 .

7. Yan, L.; Huck, W. T. S.; Zhao, X. M.; Whitesides, G. M. Patterning Thin Films of Poly(Ethylene Imine) on a Reactive Sam Using Microcontact Printing. Langmuir 1999, 15, 1208-1214.

8. Lahiri, J.; Ostuni, E.; Whitesides, G. M. Patterning Ligands on Reactive Sams by Microcontact Printing. Langmuir 1999, 15, 2055-2060.

9. Rozkiewicz, D. I.; Ravoo, B. J.; Reinhoudt, D. N. Reversible Covalent Patterning of Self-Assembled Monolayers on Gold and Silicon Oxide Surfaces. Langmuir 2005, 21, 6337-6343.

10. Rozkiewicz, D. I.; Janczewski, D.; Verboom, W.; Ravoo, B. J.; Reinhoudt, D. N. "Click" Chemistry by Microcontact Printing. Angew. Chem., Int. Ed. 2006, 45, 5292-5296.

11. Scaramuzzo, F. A.; González-Campo, A.; Wu, C. C.; Velders, A. H.; Subramaniam, V.; Doddi, G.; Mencarelli, P.; Barteri, M.; Jonkheijm, P.; Huskens, J. Pyrylium Monolayers as Amino-Reactive Platform. Chem. Commun. 2010, in press.

12. Demers, L. M.; Ginger, D. S.; Park, S. J.; Li, Z.; Chung, S. W.; Mirkin, C. A. Direct Patterning of Modified Oligonucleotides on Metals and Insulators by Dip-Pen Nanolithography. Science 2002, 296, 1836-1838.

13. Jung, H.; Dalal, C. K.; Kuntz, S.; Shah, R.; Collier, C. P. Surfactant Activated Dip-Pen Nanolithography. Nano Lett. 2004, 4, 2171-2177.

14. Matsubara, S.; Yamamoto, H.; Oshima, K.; Mouri, E.; Matsuoka, H. Fabrication of Nano-Structure by Diels-Alder Reaction. Chem. Lett. 2002, 886-887.

15. Blasdel, L. K.; Banerjee, S.; Wong, S. S. Selective Borohydride Reduction Using Functionalized Atomic 
Force Microscopy Tips. Langmuir 2002, 18, 5055-5057.

16. Long, D. A.; Unal, K.; Pratt, R. C.; Malkoch, M.; Frommer, J. Localized "Click" Chemistry through Dip-Pen Nanolithography. Adv. Mater. 2007, 19, 4471-4473.

17. Paxton, W. F.; Spruell, J. M.; Stoddart, J. F. Heterogeneous Catalysis of a Copper-Coated Atomic Force Microscopy Tip for Direct-Write Click Chemistry. J. Am. Chem. Soc. 2009, 131, 6692-+.

18. Degenhart, G. H.; Dordi, B.; Schonherr, H.; Vancso, G. J. Micro- and Nanofabrication of Robust Reactive Arrays Based on the Covalent Coupling of Dendrimers to Activated Monolayers. Langmuir 2004, 20, 6216-6224.

19. Pena, D. J.; Raphael, M. P.; Byers, J. M. "Dip-Pen" Nanolithography in Registry with Photolithography for Biosensor Development. Langmuir 2003, 19, 9028-9032.

20. Chi, Y. S.; Choi, I. S. Dip-Pen Nanolithography Using the Amide-Coupling Reaction with Interchain Carboxylic Anhydride-Terminated Self-Assembled Monolayers. Adv. Funct. Mater. 2006, 16, 1031-1036.

21. Hsu, S. H.; Reinhoudt, D. N.; Huskens, J.; Velders, A. H. Imidazolide Monolayers for Reactive Microcontact Printing. J. Mater. Chem. 2008, 18, 4959-4963.

22. Kham, K.; Guerrouache, M.; Carbonnier, B.; Lazerges, M.; Perrot, H.; Millot, M. C. Suprarnolecular Interactions between Beta-Cyclodextrin and Hydrophobically End-Capped Poly(Ethylene Glycol)S: A Quartz Crystal Microbalance Study. J. Colloid Interface Sci. 2007, 315, 800-804.

23. Park, I. K.; Von Recum, H. A.; Jiang, S. Y.; Pun, S. H. Supramolecular Assembly of Cyclodextrin-Based Nanoparticles on Solid Surfaces for Gene Delivery. Langmuir 2006, 22, 8478-8484.

24. Huskens, J. Multivalent Interactions at Interfaces. Curr. Opin. Chem. Biol. 2006, 10, 537-543.

25. Mulder, A.; Onclin, S.; Peter, M.; Hoogenboom, J. P.; Beijleveld, H.; ter Maat, J.; Garcia-Parajo, M. F.; Ravoo, B. J.; Huskens, J.; van Hulst, N. F., et al. Molecular Printboards on Silicon Oxide: Lithographic Patterning of Cyclodextrin Monolayers with Multivalent, Fluorescent Guest Molecules. Small 2005, 1, 242-253.

26. Ludden, M. J. W.; Reinhoudt, D. N.; Huskens, J. Molecular Printboards: Versatile Platforms for the Creation and Positioning of Supramolecular Assemblies and Materials. Chem. Soc. Rev. 2006, 35, 1122-1134.

27. Nijhuis, C. A.; Yu, F.; Knoll, W.; Huskens, J.; Reinhoudt, D. N. Multivalent Dendrimers at Molecular Printboards: Influence of Dendrimer Structure on Binding Strength and Stoichiometry and Their Electrochemically Induced Desorption. Langmuir 2005, 21, 7866-7876.

28. Dorokhin, D.; Hsu, S. H.; Tomczak, N.; Reinhoudt, D. N.; Huskens, J.; Velders, A. H.; Vancso, G. J. Fabrication and Luminescence of Designer Surface Patterns with Beta-Cyclodextrin Functionalized Quantum Dots Via Multivalent Supramolecular Coupling. Acs Nano 2010, 4, 137-142.

29. Maury, P.; Peter, M.; Crespo-Biel, O.; Ling, X. Y.; Reinhoudt, D. N.; Huskens, J. Patterning the Molecular Printboard: Patterning Cyclodextrin Monolayers on Silicon Oxide Using Nanoimprint Lithography and Its Application in 3d Multilayer Nanostructuring. Nanotechnology 2007, 18, -.

30. Onclin, S.; Mulder, A.; Huskens, J.; Ravoo, B. J.; Reinhoudt, D. N. Molecular Printboards: Monolayers of Beta-Cyclodextrins on Silicon Oxide Surfaces. Langmuir 2004, 20, 5460-5466. 


\title{
Chapter 6
}

\section{Porous Multilayer-coated AFM Tips for Dip-Pen Nanolithography of Proteins*}

\begin{abstract}
A simple and novel method was developed for fabricating porous multilayer-coated silicon nitride tips for dip-pen nanolithography (DPN) by using Layer-by-Layer (LbL) technique. The pore sizes can be adjusted by treating the poly4-(vinylpyridine)/ poly(acrylic acid) (P4VP/PAA) films coated onto amino-terminated monolayer ( $\mathrm{NH}_{2}$-monolayer)-functionalized AFM tip surfaces with a base solution for different periods of time. This hydrophilic porous material can easily absorb biomolecules, and provides a larger volume ink reservoir compared to a bare silicon nitride tip. Proof-of-concept of the porous AFM tip is demonstrated by using fluorescent proteins as ink molecules to fabricate protein patterns at micrometer and sub-micrometer length scale.
\end{abstract}

*Part of this chapter has been published in: Wu, C. C.; Xu, H.; Otto, C.; Reinhoudt, D. N.; Lammertink, R. G. H.; Huskens, J.; Subramaniam, V.; Velders, A. H. J. Am. Chem. Soc. 2009, 131, 7526-7527. 


\subsection{Introduction}

It has been pointed out in Chapter 2 that generally two strategies can be discerned to pattern biomolecules with the DPN technique, namely an indirect method and a direct method. ${ }^{1}$ In the indirect method first a suitable molecular functionality at a surface is prepared by DPN, which is followed by the immobilization of proteins from a solution. One great advantage of this indirect method is avoiding the denaturation of fragile biomolecules while directly writing. ${ }^{2}$ One indirect approach has been described in Chapter 4 by fabricating nickel ions patterns to immobilize proteins. A disadvantage of the indirect approach is its relatively low efficiency because one or more chemical reaction steps have to be carried out before immobilizing biomolecules.

For efficient patterning, especially for non-fragile biomolecules, directly depositing from the ink-coated tip onto a surface is simpler and more straightforward. Unlike writing small organic or inorganic molecules, transferring high molecular weight molecules, e.g. biomolecules, onto solid surfaces is much more challenging because of the slow diffusion rate, the limited amount of ink, and the difficulty of maintaining functional biological properties of molecules. ${ }^{3}$ Several modifications of tip surfaces ${ }^{2-4}$ have been reported in order to overcome these restrictions. For instance, Lee et al. modified the tip surface with a PEG monolayer in order to facilitate the diffusion process from the tip to the substrate. ${ }^{2}$ Choi et al. utilized the polymerization method to grow a thin porous film on the tip surface and create a sponge-like tip. ${ }^{3}$ More recently, Senesi et al. utilized the porous property of agarose to encapsulate biomolecules for performing DPN experiments. ${ }^{5}$

In this chapter, a new and simple approach is presented, using Layer-by-Layer (LbL) self-assembly of poly4-(vinylpyridine)/poly(acrylic acid) (P4VP/PAA), to form hydrophilic porous structures on the surface of $\mathrm{Si}_{3} \mathrm{~N}_{4}$ AFM tips. This porous thin film coated on an AFM tip can act as an ink reservoir with increased volume. LbL self-assembly has increasingly attracted attention due to its simplicity, versatility, and systematic control over the thickness and underlying structure of the resulting layer. ${ }^{6-8}$ By immersing the LbL multilayer in solutions with different $\mathrm{pH}$ or ionic strengths, porous structures at micrometer and nanometer length scales have been formed attributing to the re-conformation or aggregation of remained polymer on the surface. ${ }^{9}{ }^{10}$ An attractive feature of this method is that the pore size and the thickness of the porous films can be tuned by altering parameters such as polymer concentrations, $\mathrm{pH}$ value of the alkaline solutions, and duration of base treatment. Such porous structures have also been applied onto poly(dimethylsiloxane) (PDMS) stamps to function as a reservoir for multiple 
microcontact printing $(\mu \mathrm{CP})$ of immunoglobulin $\mathrm{G}$ (IgG) onto a amino-terminated monolayer-functionalized glass substrate. ${ }^{11}$

\subsection{Results and Discussion}

\subsubsection{Fabrication and characterization of porous multilayer-coated AFM tip}

LbL films are generally constructed via electrostatic interactions ${ }^{7,8,12,13}$ between two kinds of polymers. Other interactions, e.g. covalent bonding, ${ }^{14}$ hydrogen bonding, ${ }^{15-18}$ and specific molecular recognition ${ }^{19}$ have also been utilized as the driving force for LbL assembly. The preparation of porous multilayer-coated AFM tips in principle followed the procedure published by Fu et al., and is shown in Scheme 6.1. ${ }^{9}$ To affix the polymer on the cantilever surface, a cleaned bare $\mathrm{Si}_{3} \mathrm{~N}_{4}$ cantilever was first functionalized with an amino-terminated self-assembled monolayer by gas-phase deposition of $N$-[3-(trimethoxysilyl)propyl]-ethylenediamine (TPEDA monolayer). This TPEDA monolayer allows the interaction with the polymer via hydrogen bonding. The TPEDA monolayer-functionalized AFM cantilevers were alternately incubated in poly (4-vinylpyridine) (P4VP) and poly(acrylic acid) (PAA) methanol solutions for assembling LbL15 films based on hydrogen bonding between the pyridine and carboxylic acid groups (15 denotes the number of P4VP/PAA bilayers). These LbL15-functionalized cantilevers were subsequently immersed into a $0.1 \mathrm{~N}$ of $\mathrm{KOH}$ solution for 5,10 or 15 minutes to dissolve the PAA in the multilayer. This dissolution phenomenon of PAA in alkaline solution has been reported previously showing the release of PAA did not remove whole multi-layers away from the tip surface. Due to the poor solubility of P4VP in the alkaline solution, the remaining P4VP led to the formation of porous structures on the cantilever surfaces. ${ }^{9,20}$ 


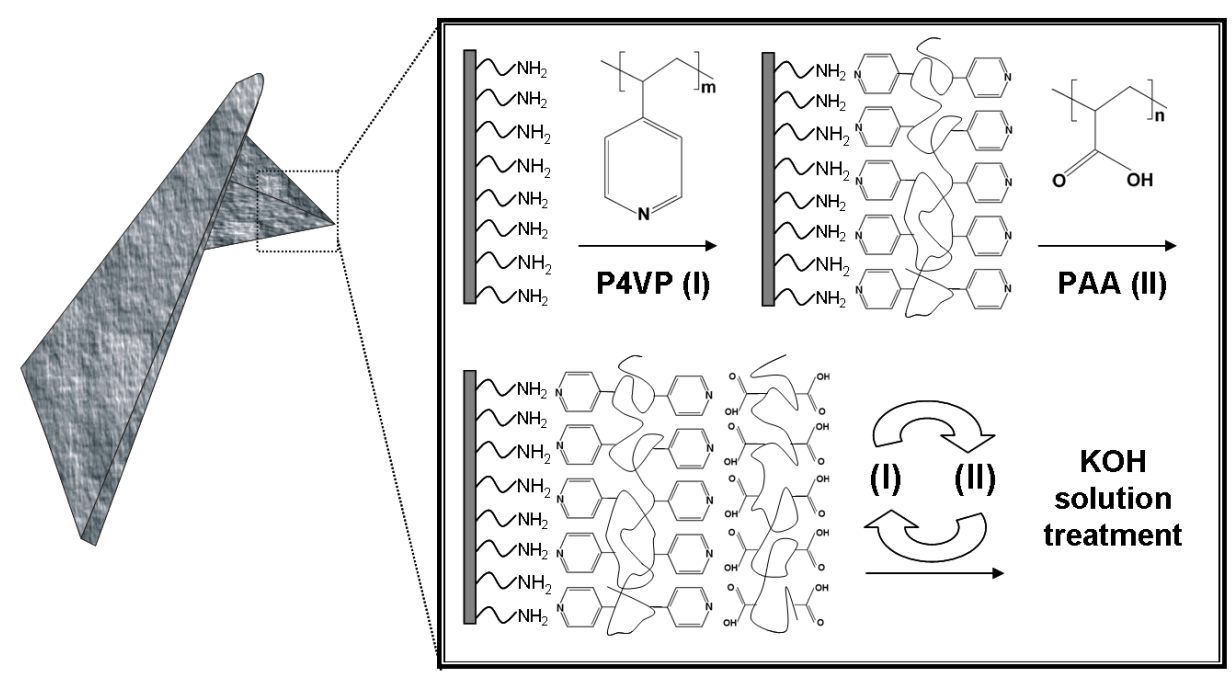

Scheme 6.1. Preparation procedure of a porous LbL AFM tip.

The porous LbL15 tips were characterized by scanning electron microscopy (SEM) and atomic force microscopy (AFM). (Figure 6.1a) The SEM images of the porous LbL15 tips, fabricated by varying the duration of the treatment in $\mathrm{KOH}$ solution, are shown in Figure 6.1c-e. The corresponding supporting chip areas of the cantilevers used for DPN were examined by AFM in tapping mode, and are shown in Figure 6.1f-h. Larger pore sizes and more apparent porous network structures were obtained upon longer treatment with alkaline solution. The pore sizes ranged from $\sim 50 \mathrm{~nm}$ to a few hundreds of $\mathrm{nm}$ as determined by AFM. The thickness of the porous film is between 20 and $30 \mathrm{~nm}$. The SEM images also show that the LbL structures make the tip blunter than the $\mathrm{Si}_{3} \mathrm{~N}_{4}$ tip functionalized with TPEDA monolayer (Figure 6.1b). Nevertheless, the radius of the porous tip is $\sim 100 \mathrm{~nm}$ which is still considered within the acceptable length scale for performing DPN experiments. 

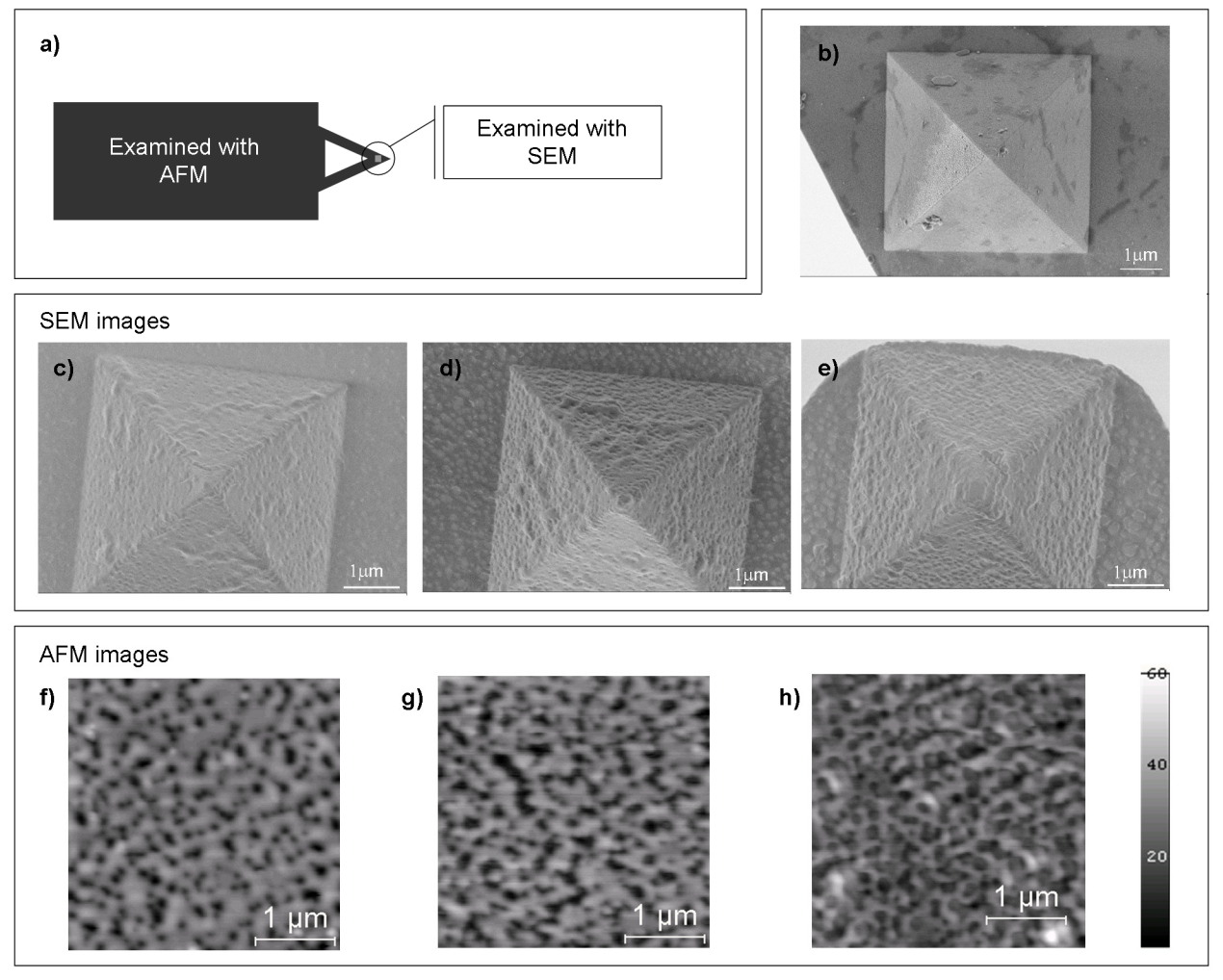

Figure 6.1. (a) Schematic illustration the position of the cantilever examined with SEM and $A F M$, respectively. (b) SEM image of a $\mathrm{Si}_{3} \mathrm{~N}_{4}$ AFM tip functionalized with TPEDA monolayer. (c), (d) and (e) SEM images of LbL15-functionalized tips treated with KOH solution for 5, 10 and 15 minutes, respectively. (f), (g) and (h) tapping mode AFM height images of the porous cantilever treated with $\mathrm{KOH}$ solution for 5, 10 and 15 minutes, respectively.

To prove that the prepared porous tip could encapsulate more biomolecular ink than a bare tip, a bare $\mathrm{Si}_{3} \mathrm{~N}_{4}$ AFM tip and a porous LbL15 tip were inked with a small drop of His-tagged tetrameric reef coral fluorescent protein (His-DsRed) solution $(2 \mu \mathrm{M})$ placed onto an octadecyltrichlorosilane (OTS monolayer)-functionalized glass slide for $\sim 10$ minutes. The OTS monolayer is hydrophobic and therefore it avoids the spreading of the protein solution. After inking, the tips were blown dry with $\mathrm{N}_{2}$ and were imaged with fluorescence microscopy (Figure 6.2). It can be seen that the fluorescence intensity of the 
porous LbL15 tip is significantly higher than that of the bare tip. This result proves that more fluorescent proteins are encapsulated into the porous tip, which effectively acts as a sponge.
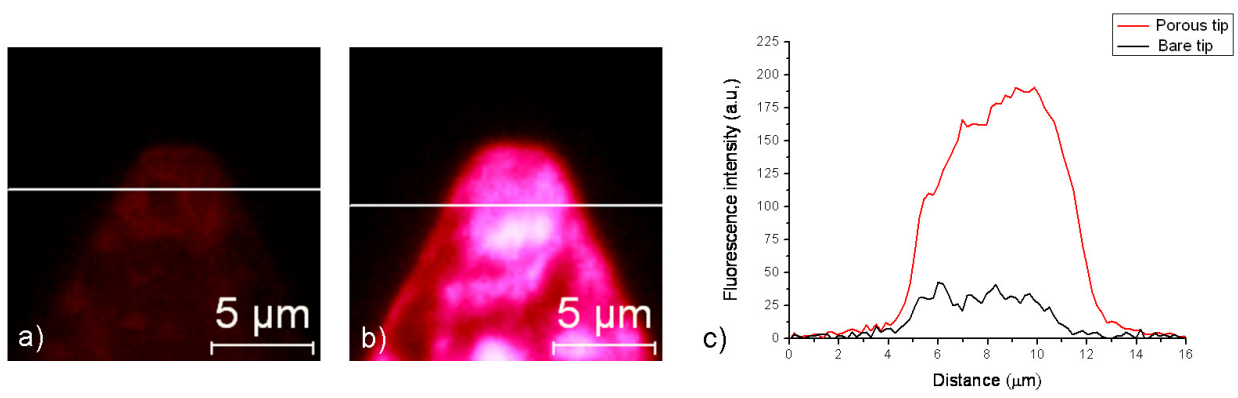

Figure 6.2. Fluorescence images of (a) a bare AFM tip and (b) a porous LbL15 tip, dipped into His-Ds Red solution (2 $\mu M)$ for 10 minutes and then dried with $N_{2}$. The exposure time of both images is $100 \mathrm{~ms}$. (c) The intensity profiles of the line shown in (a) and (b).

\subsubsection{Fabrication of protein patterns with multilayer-coated AFM tip}

Porous LbL tips were examined for their potential application in transferring biomolecules via DPN. Proof-of concept experiments were performed by generating His-tagged visible fluorescent protein (VFP) patterns on TPEDA monolayer- or nickel(II)nitrilotriacetic acid-terminated (Ni-NTA) monolayer-functionalized glass substrates through electrostatic interactions or the formation of metal ion-imidazole bonds, respectively. Two proteins with different molecular weights, His-tagged enhanced green fluorescent protein (His-EGFP, $\sim 27 \mathrm{kDa}$ ) and His-tagged tetrameric reef coral fluorescent protein (His-DsRed, $100 \mathrm{kDa}$ ), were used. The intrinsic fluorescence of the VFPs is an indicator of structural and functional integrity, since structurally damaged or misfolded proteins do not fluoresce.

In the procedure of inking metal ions, i.e. $\mathrm{Ca}(\mathrm{II})$ and $\mathrm{Ni}(\mathrm{II})$, as described in Chapter 3 and Chapter 4 respectively, the commercial $\mathrm{Si}_{3} \mathrm{~N}_{4}$ AFM tips were fully incubated into metal ion solutions for coating. Since proteins are much bigger molecules compared to metal ions, the inking procedure was modified, in order to avoid the proteins adhering to 
the backside of the cantilever. This adsorption may obstruct the detection of the laser deflection signal and affect performing further DPN experiments. Scheme 6.2 exhibits how a porous LbL15 tip inked with proteins. A small drop of protein solution $(\sim 2 \mu \mathrm{l})$ was placed onto the OTS monolayer-functionalized glass substrate right above an objective lens. To prevent the ink solution from drying during the inking process, a thin layer of wet tissue paper was placed surrounding the protein drop. Subsequently, a porous LbL15 tip mounted on the AFM head was very gently brought to touch the protein drop for $\sim 10$ minutes and then carefully dried with $\mathrm{N}_{2}$.
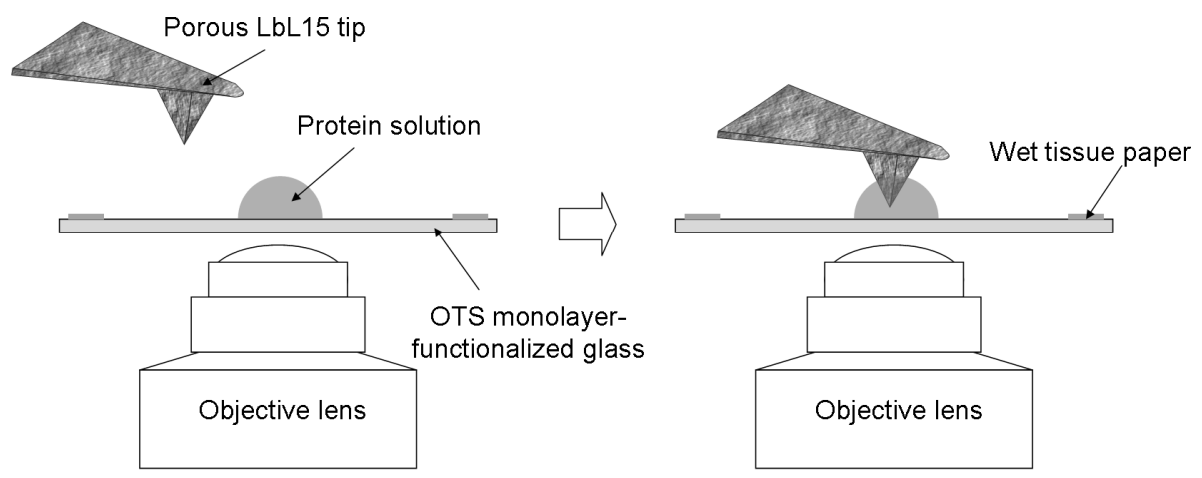

Scheme 6.2. Illustration of the procedure of inking proteins with a porous LbL15 tip. The schematic is not to scale.

\subsubsection{His-EGFP patterns}

A His-EGFP-impregnated porous tip was brought into contact with a TPEDA monolayer-functionalized surface to write a $25 \mu \mathrm{m}$ wide square in one move with an atomic force fluorescence microscope $(\mathrm{AFFM})^{21}$ at $\sim 60 \%$ relative humidity $(\mathrm{RH})$ with a writing speed of $25 \mu \mathrm{m} / \mathrm{s}$. The resulting fluorescent pattern was immediately imaged in situ by the AFFM. Without a re-inking process, the same His-EGFP square pattern was generated more than 20 times, as shown in Figure 6.3. This series of results again successfully demonstrates that the porous LbL tip acts as a sponge-like reservoir for encapsulating large amounts of aqueous protein solution, maintaining the protein functionality and enabling ink release during the short tip-surface contact period. It can 
also be observed that the protein lines became narrower while proceeding with the DPN experiments. This phenomenon indicates that there was a depletion of ink molecules from the tip as the DPN experiments progressed.
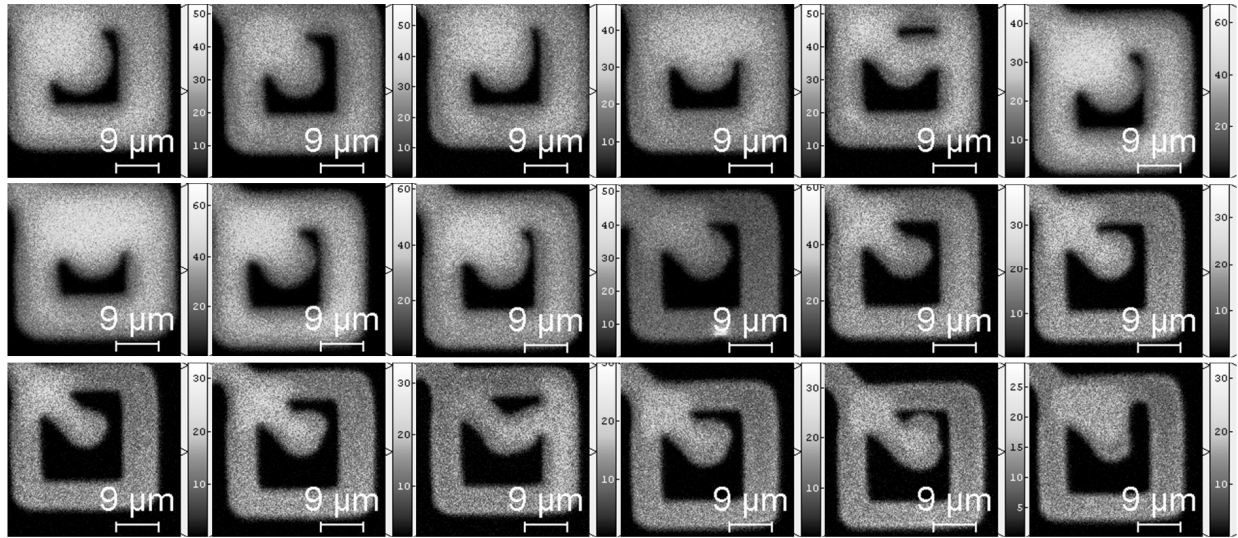

Figure 6.3. Fluorescence images of His-EGFP square patterns that were generated successively on TPEDA monolayer from left to right and from top to bottom (zigzag). The scale of the color bar for the different images is not uniform.

\subsubsection{His-DsRed patterns}

To further demonstrate the general applicability of the porous LbL15 tips, His-DsRed, another type of protein with 4 times higher molecular weight than His-EGFP, was also used to create patterns on the Ni-NTA monolayer-functionalized glass substrates. In order to obtain the protein patterns at sub-micron scale, a series of writing experiments at different $\mathrm{RH}$ was performed since $\mathrm{RH}$ has been reported as one of the important factors in determining the resolution in DPN experiments. ${ }^{22}$ A His-DsRed-impregnated porous tip was first brought into contact with a Ni-NTA monolayer-functionalized surface to generate a pattern at $\sim 60 \% \mathrm{RH}$. Immediately after writing, a fluorescent pattern at micron scale was observed and is shown in Figure 6.4a. The same tip, without re-inking, was subsequently used to generate some dot and line patterns at $\sim 30 \%$ RH. Figure $6.4 \mathrm{~b}$ and $6.4 \mathrm{c}$ demonstrate that fluorescent patterns at sub-micron scale were successfully obtained. The fluorescence intensity shown in Figure $6.4 \mathrm{~b}$ is lower than that shown in Figure 6.4a, indicating that less amount of proteins were deposited on the surface. To confirm that the 
protein patterns fabricated at sub-micron scale were due to the decrease of RH instead of the depletion of ink molecules, the same tip without re-inking was used again to generate patterns at $\sim 45 \%$ RH. In Figure $6.4 \mathrm{~d}$, fluorescent features at micron scale were observed again. This process can be repeated as shown in Figure $6.4 \mathrm{e}$ and $6.4 \mathrm{f}$ proving that controlling $\mathrm{RH}$ is a significant issue for obtaining protein patterns at the desired length scale. Since our interest is to generate features at sub-micron scale in order to obtain high-density protein patterns, lower RH was applied for the DPN experiments.

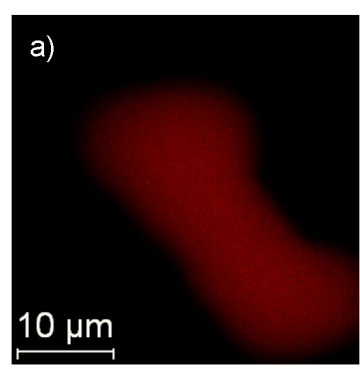

$\mathrm{RH}: \sim 60 \%$

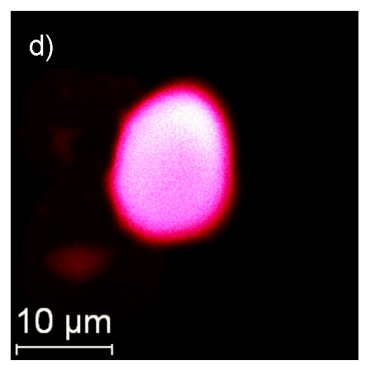

$\mathrm{RH}: \sim 45 \%$

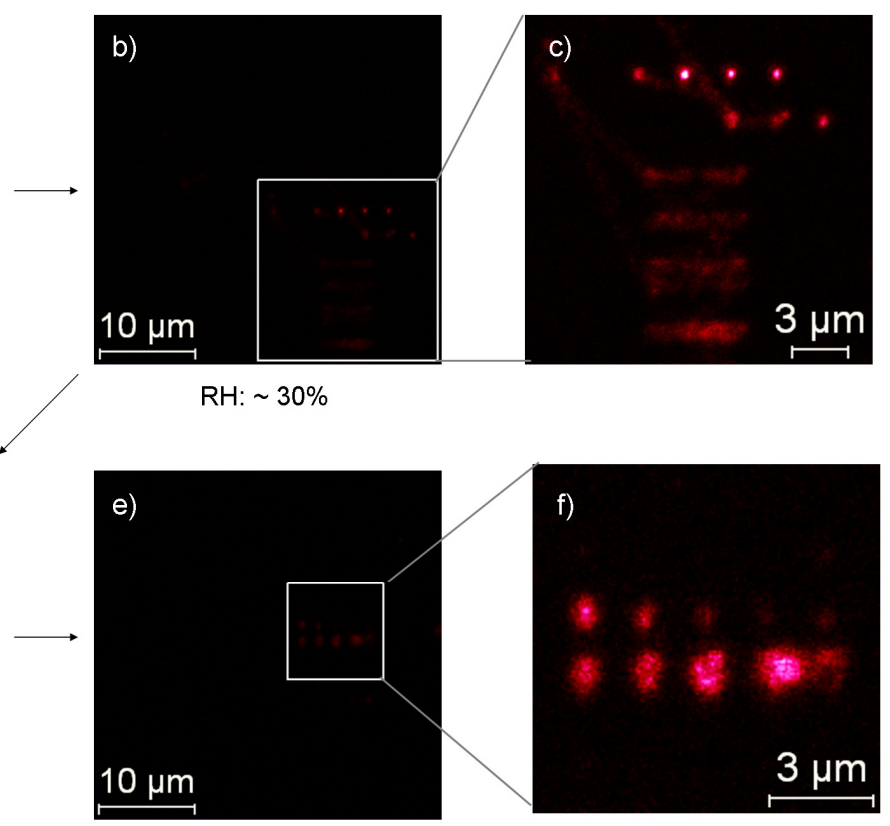

$\mathrm{RH}: \sim 30 \%$

Figure 6.4. (a), (b), (d) and (e) Fluorescence images of His-DsRed patterns which were generated successively without reinking the tip on Ni-NTA monolayer-functionalized substrates at varied relative humidity. The color scale of (a), (b), (d)and (e) is the same. (c) and $(f)$ are the zoom-in fluorescence image of the white square indicated in (b) and (e), respectively. 
A porous tip impregnated with His-DsRed was brought into contact with the surface for a given contact time of 10 seconds at $~ 35 \% \mathrm{RH}$. The inked porous tip was used to successively generate 42 spots and was retracted after depositing each dot. No re-inking process occurred during the entire writing sequence. Tapping mode AFM revealed further topographical information about the protein patterns. The fluorescence and topographical information about the protein dot patterns are shown in Figure $6.5 \mathrm{a}$ and $6.5 \mathrm{~b}$ respectively. To avoid contamination, a new $\mathrm{Si}_{3} \mathrm{~N}_{4}$ AFM tip was used for imaging. Despite repeated writing, it was observed that the fluorescence intensity does not decrease gradually, suggesting that the ink flows from the distal end to the tip. Figure $6.5 \mathrm{c}$ and $6.5 \mathrm{~d}$ showed that the diameters of His-DsRed arrays were between $200 \mathrm{~nm}$ and $300 \mathrm{~nm}$ and did not vary much. The height data suggest that multilayers of proteins were deposited on the surface. Another porous tip impregnated with His-DsRed was utilized to generate two continuous line patterns at $\sim 35 \% \mathrm{RH}$ with a writing speed of $5 \mu \mathrm{m} / \mathrm{s}$. The resulting fluorescent pattern is shown in Figure 6.6 and also shows that the fluorescence intensity did not decrease gradually despite writing continuously, consistent with the data in Figure $6.5 \mathrm{a}$.

a)

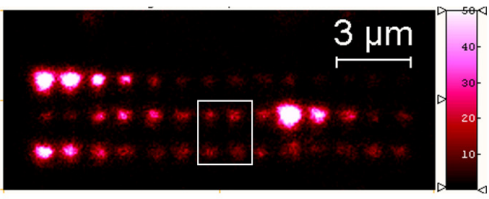

c)

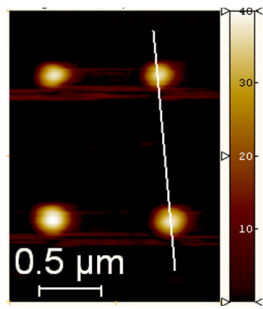

d) b)
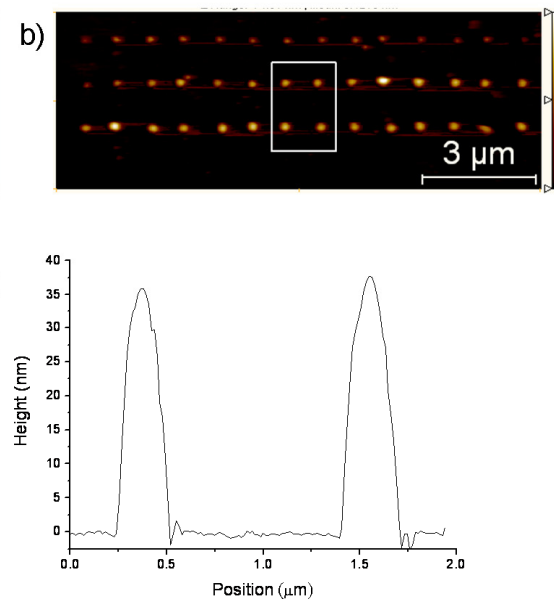

Figure 6.5. (a) Fluorescence images of His-DsRed square patterns acquired immediately after DPN with a porous LbL15 tip. The dot patterns were successively generated from left to right and from top to bottom (zigzag). (b) AFM tapping mode image in height of His-DsRed dot patterns shown in (a). (c) Zoom-in image of the indicated area shown in (b). (d) Topography information in height of the line indicated in (c). 


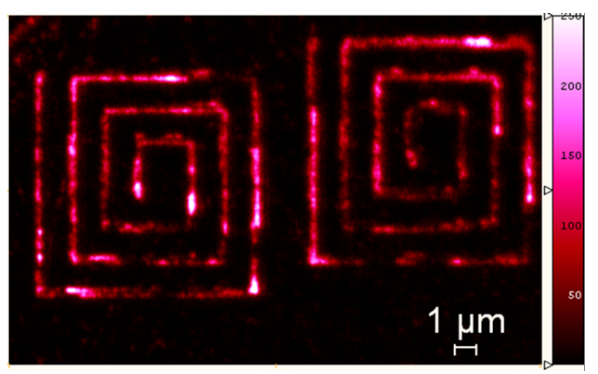

Figure 6.6. Fluorescence images of His-DsRed continuous line patterns acquired immediately after DPN with a porous LbL15 tip. The lines were written counterclockwise from outside to inside and the left pattern was created first.

\subsubsection{Examining the co-deposition of the porous material}

A potential risk was that the porous material coated on an AFM tip might be simultaneously deposited onto the substrate while performing DPN experiments. Therefore, a porous LbL15 tip before use and after writing with His-DsRed and subsequent rinsing with MilliQ water were examined by SEM, shown in Figure 6.7a and $6.7 \mathrm{~b}$, respectively. Although the tip was rinsed with MilliQ water, it was very difficult to remove all the remaining His-DsRed and a coating layer (remaining proteins and porous structures) was observed in Figure 6.7b. It can be seen that there was no apparent polymer release from the tip surface after writing. From Figure 6.7, we cannot conclusively exclude this possibility of co-deposition during the DPN experiments and therefore another experiment was performed for a further check.
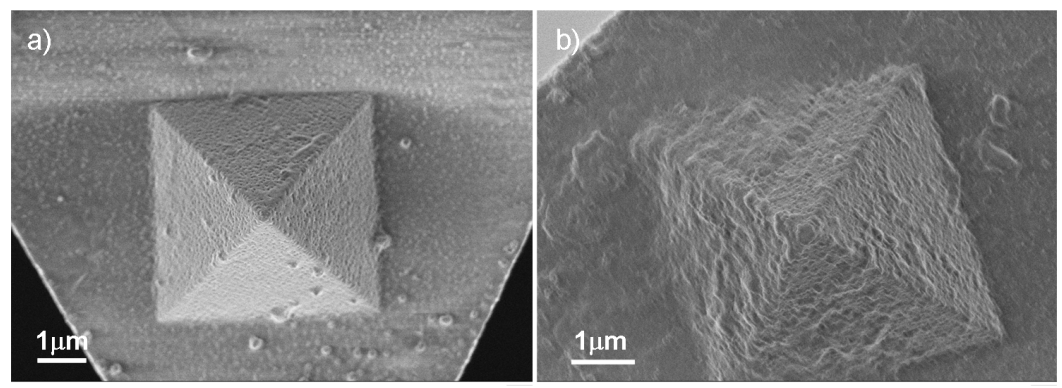

Figure 6.7. SEM images of an LbL15-functionalized porous tip, (a) before and (b) after writing His-DsRed. 
A cleaned glass substrate was first imaged in contact mode AFM, shown in Figure 6.8a, before performing DPN experiments. A porous LbL15 tip was incubated into MilliQ water for $\sim 10$ minutes and then blow-dried for writing. Subsequently, several line and dot patterns with varied writing speeds and tip-surface contact time were generated onto the cleaned glass substrate with the porous tip inked with MilliQ water. After writing, a new $\mathrm{Si}_{3} \mathrm{~N}_{4}$ AFM tip was used to image the written area shown in Figure 6.8b. There were no obvious features exhibited in Figure $6.8 \mathrm{~b}$, indicating the co-deposition of the porous material can be ignored.

a)

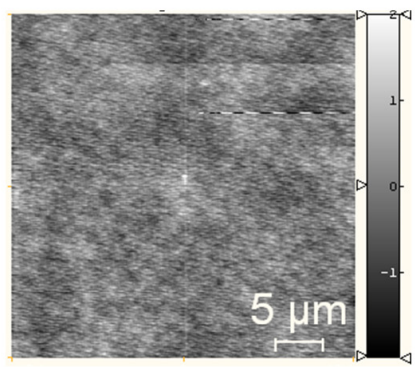

b)

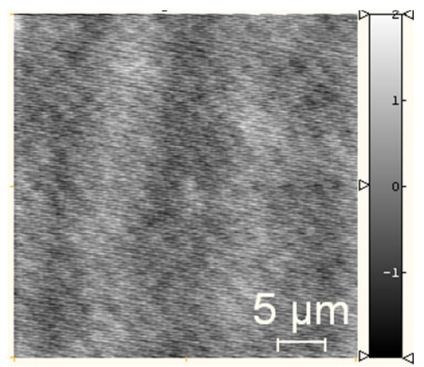

Figure 6.8. AFM contact mode image in height of a cleaned glass substrate (a) before and (b) after writing with a porous LbL15 tip inked with MilliQ water.

\subsection{Conclusions}

In summary, a simple method for fabricating surface-modified nanoporous AFM tips using the LbL technique has been presented in this chapter. The pore sizes of the porous film can easily be adjusted by varying the incubation time of the LbL15 tips in alkaline solution. We have successfully demonstrated that aqueous fluorescent protein solutions can easily be absorbed into the porous LbL tip while retaining the biological activity of the proteins for extended periods. Furthermore, the pore structures provide a large-volume ink reservoir for DPN experiments. His-EGFP and His-DsRed patterns at micrometer and submicrometer scales were successfully fabricated with the LbL15 porous tips. Different parameters, e.g. the molecular weight of P4VP and the number of bilayers, for tuning the pore sizes and properties will be described in more detail in Chapter 7. 


\subsection{Experimental}

\section{General procedures.}

All glassware used to prepare the monolayers was cleaned by sonicating for 60 minutes in a $2 \% \mathrm{v} / \mathrm{v}$ Hellmanex II solution in Milli-Q water, and then rinsed thoroughly with MilliQ water, and dried in an ambient environment. Microscope glass slide substrates and $\mathrm{Si}_{3} \mathrm{~N}_{4}$ AFM cantilevers (NP, Veeco) were cleaned in piranha solution for 20 minutes and 1 minute, respectively, (concentrated $\mathrm{H}_{2} \mathrm{SO}_{4}$ and $33 \%$ aqueous $\mathrm{H}_{2} \mathrm{O}_{2}$ in a 3:1 ratio). [Warning: Piranha solution should be handled with caution: it has been reported to detonate unexpectedly.] The glass slides and cantilevers were then rinsed several times with Milli-Q water, and dried gently under a nitrogen stream before forming the monolayer.

\section{Monolayers preparation.}

Preparation of the amino terminated monolayer (TPEDA). The preparation of cleaned microscope glass slide substrates and $\mathrm{Si}_{3} \mathrm{~N}_{4}$ AFM cantilevers were placed in a desiccator under vacuum overnight for the gas-phase evaporation of $N$-[3-(trimethoxysilyl)propyl]ethylenediamine to form amino-terminated monolayer (TPEDA monolayer). After the TPEDA monolayer-functionalized substrates and cantilevers were taken out from the desiccator, they were rinsed carefully with ethanol and dichloromethane to remove physisorbed materials and then dried under a stream of $\mathrm{N}_{2}$. In order to obtain more stable TPEDA monolayer, the cantilevers and substrates were placed in an oven at $60^{\circ} \mathrm{C}$ for 1 hour before performing experiments.

Preparation of OTS monolayer. ${ }^{23}$ Cleaned microscope glass slide substrates immersed in a distilled toluene solution of $1 \mathrm{mM}$ octadecyltrichlorosilane (OTS) for 1 hour. After the substrates were taken from the solution, they were rinsed carefully to remove excess physisorbed materials and then dried under a stream of $\mathrm{N}_{2}$.

Preparation of the Ni-NTA monolayer. ${ }^{24}$ The glass substrates functionalized with Ni-NTA monolayers were prepared by following the procedures described in Chapter 4 .

\section{Fabrication of multilayer-coated porous tip. ${ }^{9}$}

Poly(acrylic acid) $50 \mathrm{wt} \%$ solution in water MW 5000 was first freeze dried to obtain pure poly(acrylic acid) (PAA). Poly (4-vinylpyridine) (P4VP), MW 50000 and PAA were dissolved in methanol individually to obtain $1 \mathrm{mg} / \mathrm{ml} \mathrm{P4VP}$ and PAA methanol solution. The TPEDA monolayer-functionalized AFM cantilevers were first incubated into P4VP solution for 10 minutes and then rinsed several times with methanol before incubating into PAA solution for 10 minutes. This process was repeated for 15 times to obtained Layer-by-Layer (LbL)-functionalized cantilevers. The LbL-functionalized cantilevers were subsequently immersed into $\mathrm{KOH}$ solution of $0.1 \mathrm{~N}$ for varied periods of time to obtain porous structure coated cantilevers with different sizes of pores. The porous cantilevers were rinsed with Milli-Q water and then dried very gently under a stream of $\mathrm{N}_{2}$. 


\section{Analysis of porous structures.}

The surface structures of porous cantilevers were characterized by a scanning electron microscope (SEM) (Zeiss) and a custom-built atomic force microscope (AFM) in tapping mode equipped with a $\mathrm{Si}_{3} \mathrm{~N}_{4}$ AFM cantilever (MSCT, Veeco) with a nominal spring constant of $0.54 \mathrm{~N} / \mathrm{m}$.

\section{Acquisition of fluorescent images.}

The porous LbL15 tip and the bare $\mathrm{Si}_{3} \mathrm{~N}_{4}$ tip inked with His-DsRed were placed onto a cleaned glass substrate and then examined with a fluorescence microscope (TE2000-U, Nikon) equipped with a mercury lamp as light source and a digital camera (Nikon digital Sight DS-Qi1Mc). To obtain fluorescence images of proteins adhered to tip surfaces, the filter cube with green excitation light $(\lambda=510 / 40 \mathrm{~nm})$, red emission light $(\lambda=617 / 73 \mathrm{~nm})$ and dichroic $(\lambda=562 \mathrm{~nm})$ was used. The obtained fluorescence images were analyzed with the commercial software SPIP (Image Metrology, Version 4.4.3.0).

\section{Dip pen nanolithography with multilayer-coated porous tips and visualization with AFFM.}

For DPN experiments, the porous LbL tips treated with alkaline solution for 15 minutes were used. Immediately before a DPN experiment, $\sim 3 \mu \mathrm{l}$ of $10 \mathrm{mM}$ Tris- $\mathrm{HCl}$ buffer ( $\mathrm{pH}$ 7.5) containing $5 \mu \mathrm{M}$ His-EGFP or $2 \mu \mathrm{M}$ His-DsRed was placed onto OTS monolayer-functionalized glass slide. The porous cantilever mounted on the AFM head was very carefully brought to immerse into the drop of fluorescent proteins $\sim 10$ minutes and then dried very gently under a stream of $\mathrm{N}_{2}$. All DPN experiments were operated inside a custom-built chamber with a controlled humidity system at a temperature between 20 and $22{ }^{\circ} \mathrm{C}$. After creating His-EGFP or His-DsRed patterns on the surface, the fluorescent images of deposited fluorescent proteins were obtained by exciting molecules with $488 \mathrm{~nm}$ line of an Argon-ion laser (163-D Laser System, Spectra Physics) and recording the emission of the fluorescence by an avalanche photodiode (APD) (SPCM-AQR-14, Perkin Elmer Optoelectronics). To obtain AFM topography information of the protein patterns, tapping mode was used. The original ink-coated porous tip was replaced by a new $\mathrm{Si}_{3} \mathrm{~N}_{4}$ AFM tip (MSCT, Veeco) with a nominal spring constant of $0.54 \mathrm{~N} / \mathrm{m}$ to prevent contamination of the patterned areas during subsequent imaging.. Fluorescence and topographic images were analyzed and prepared with the commercial software SPIP (Image Metrology, Version 4.4.3.0).

\subsection{Acknowledgements}

Dr. Huaping $\mathrm{Xu}$ is acknowledged for generously sharing his knowledge about preparing the porous multilayer and for valuable suggestions. 


\subsection{References}

1. Christman, K. L.; Enriquez-Rios, V. D.; Maynard, H. D. Nanopatterning Proteins and Peptides. Soft Matter 2006, 2, 928-939.

2. Lee, K. B.; Lim, J. H.; Mirkin, C. A. Protein Nanostructures Formed Via Direct-Write Dip-Pen Nanolithography. J. Am. Chem. Soc. 2003, 125, 5588-5589.

3. Choi, D. S.; Yun, S. H.; An, Y. C.; Lee, M. J.; Kang, D. G.; Chang, S. I.; Kim, H. K.; Kim, K. M.; Lim, J. H. Nanopatterning Proteins with a Stamp Tip for Dip-Pen Nanolithography. Biochip J. 2007, 1, 200-203.

4. Lim, J. H.; Ginger, D. S.; Lee, K. B.; Heo, J.; Nam, J. M.; Mirkin, C. A. Direct-Write Dip-Pen Nanolithography of Proteins on Modified Silicon Oxide Surfaces. Angew. Chem., Int. Ed. 2003, 42, 2309-2312.

5. Senesi, A. J.; Rozkiewicz, D. I.; Reinhoudt, D. N.; Mirkin, C. A. Agarose-Assisted Dip-Pen Nanolithography of Oligonucleotides and Proteins. Acs Nano 2009, 3, 2394-2402.

6. Decher, G. Fuzzy Nanoassemblies: Toward Layered Polymeric Multicomposites. Science 1997, 277, 1232-1237.

7. Decher, G.; Hong, J. D.; Schmitt, J. Buildup of Ultrathin Multilayer Films by a Self-Assembly Process.3. Consecutively Alternating Adsorption of Anionic and Cationic Polyelectrolytes on Charged Surfaces. Thin Solid Films 1992, 210, 831-835.

8. Decher, G.; Hong, J. D. Buildup of Ultrathin Multilayer Films by a Self-Assembly Process.2. Consecutive Adsorption of Anionic and Cationic Bipolar Amphiphiles and Polyelectrolytes on Charged Surfaces. PCCP 1991, 95, 1430-1434.

9. Fu, Y.; Bai, S. L.; Cui, S. X.; Qiu, D. L.; Wang, Z. Q.; Zhang, X. Hydrogen-Bonding-Directed Layer-by-Layer Multilayer Assembly: Reconformation Yielding Microporous Films. Macromolecules 2002, 35, 9451-9458.

10. Kim, B. Y.; Bruening, M. L. Ph-Dependent Growth and Morphology of Multilayer Dendrimer/Poly(Acrylic Acid) Films. Langmuir 2003, 19, 94-99.

11. Xu, H. P.; Gomez-Casado, A.; Liu, Z. H.; Reinhoudt, D. N.; Lammertink, R. G. H.; Huskens, J. Porous Multilayer-Coated Pdms Stamps for Protein Printing. Langmuir 2009, 25, 13972-13977.

12. Decher, G.; Hong, J. D. Buildup of Ultrathin Multilayer Films by a Self-Assembly Process.1. Consecutive Adsorption of Anionic and Cationic Bipolar Amphiphiles on Charged Surfaces. Makromolekulare Chemie-Macromolecular Symposia 1991, 46, 321-327.

13. Ariga, K.; Hill, J. P.; Ji, Q. M. Layer-by-Layer Assembly as a Versatile Bottom-up Nanofabrication Technique for Exploratory Research and Realistic Application. PCCP 2007, 9, 2319-2340.

14. Fang, M. M.; Kaschak, D. M.; Sutorik, A. C.; Mallouk, T. E. A "Mix and Match" Ionic-Covalent Strategy for Self-Assembly of Inorganic Multilayer Films. J. Am. Chem. Soc. 1997, 119, 12184-12191.

15. Stockton, W. B.; Rubner, M. F. Molecular-Level Processing of Conjugated Polymers.4. Layer-by-Layer Manipulation of Polyaniline Via Hydrogen-Bonding Interactions. Macromolecules 1997, 30, 2717-2725.

16. Kharlampieva, E.; Kozlovskaya, V.; Sukhishvili, S. A. Layer-by-Layer Hydrogen-Bonded Polymer Films: From Fundamentals to Applications. Adv. Mater. 2009, 21, 3053-3065.

17. Wang, L. Y.; Fu, Y.; Wang, Z. Q.; Fan, Y. G.; Zhang, X. Investigation into an Alternating Multilayer Film of Poly(4-Vinylpyridine) and Poly(Acrylic Acid) Based on Hydrogen Bonding. Langmuir 1999, 15, 1360-1363.

18. Wang, L. Y.; Wang, Z. Q.; Zhang, X.; Shen, J. C.; Chi, L. F.; Fuchs, H. A New Approach for the 
Fabrication of an Alternating Multilayer Film of Poly(4-Vinylpyridine) and Poly(Acrylic Acid) Based on Hydrogen Bonding. Macromol. Rapid Commun. 1997, 18, 509-514.

19. Spaeth, K.; Brecht, A.; Gauglitz, G. Studies on the Biotin-Avidin Multilayer Adsorption by Spectroscopic Ellipsometry. J. Colloid Interface Sci. 1997, 196, 128-135.

20. Xu, H.; Gomez-Casado, A.; Reinhoudt, D. N.; Lammertink, R. G. H.; Huskens, J., Porous Multilayer-Coated Pdms Stamps for Protein Printing. In Langmuir, 2009.

21. Kassies, R.; Van der Werf, K. O.; Lenferink, A.; Hunter, C. N.; Olsen, J. D.; Subramaniam, V.; Otto, C. Combined Afm and Confocal Fluorescence Microscope for Applications in Bio-Nanotechnology. $J$. Microsc. 2005, 217, 109-116.

22. Ginger, D. S.; Zhang, H.; Mirkin, C. A. The Evolution of Dip-Pen Nanolithography. Angew. Chem., Int. Ed. 2004, 43, 30-45.

23. Kulkarni, S. A.; Mirji, S. A.; Mandale, A. B.; Gupta, R. P.; Vijayamohanan, K. P. Growth Kinetics and Thermodynamic Stability of Octadecyltrichlorosilane Self-Assembled Monolayer on Si (100) Substrate. Mater. Lett. 2005, 59, 3890-3895.

24. Paik, H. J.; Kim, Y. R.; Orth, R. N.; Ober, C. K.; Coates, G. W.; Batt, C. A. End-Functionalization of Poly(3-Hydroxybutyrate) Via Genetic Engineering for Solid Surface Modification. Chem. Commun. 2005, 1956-1958. 


\title{
Chapter 7
}

\section{Ink Loading of Porous Multilayer-coated AFM Tips*}

\begin{abstract}
This chapter describes the investigation of the morphology variations of porous multilayers-coated silicon nitride AFM tips using the Layer-by-Layer (LbL) technique described in Chapter 6. The porous films were prepared by systematically varying different parameters, including the molecular weight of poly(4-vinylpyridine) (P4VP), the number of assembled layers, and the duration of $\mathrm{KOH}$ solution treatment. The morphology variations were characterized using scanning electron microscopy (SEM) and atomic force microscopy (AFM). The porous tips prepared under various conditions were inked with two kinds of fluorescent proteins, i.e. histidine-tagged enhanced green fluorescent protein (His-EGFP, $\sim 27 \mathrm{kDa}$ ) and histidine-tagged tetrameric reef coral fluorescent protein (His-DsRed, $\sim 100 \mathrm{kDa}$ ), to study the ink reservoir properties of the porous films. The different LbL conditions yielded tips with different ink loading capacities, which indicates tailoring these variable parameters can be of significant value in generating porous tips capable of performing long-term dip-pen nanolithography (DPN) writing.
\end{abstract}

\footnotetext{
${ }^{*}$ Manuscript in preparation.
} 


\subsection{Introduction}

Chapter 6 described a simple approach using Layer-by-Layer (LbL) self-assembly techniques to alternately grow multiple poly(4-vinylpyridine) (P4VP) and poly(acrylic acid) (PAA) layers on silicon nitride AFM tip surfaces based on hydrogen bonding. ${ }^{1-4}$ By immersing the LbL multilayer into alkaline solutions for different times, hydrophilic porous structures at micrometer and nanometer length scales were formed. ${ }^{1,5,6}$ These porous thin films can act as a reservoir with increased volume for the ink molecules. ${ }^{5}$ In addition to the duration of post-base treatment, many other factors that have an influence on the formation of porous structures such as $\mathrm{pH}$ values of alkaline solutions, ${ }^{1}$ surface properties of substrates, ${ }^{7}$ type of polymers, ${ }^{8,9}$ type and molecular weights of polymers ${ }^{10}$ and the number of bilayers grown on tip surfaces ${ }^{1}$ have been described. Bai et al. have demonstrated that the strong attractive interaction between P4VP and substrates has led to slower variation of microporous morphology. ${ }^{7}$ Zhang et al. assembled PAA and poly(2-vinylpyridine) (P2VP) of different molecular weight onto amino-terminated monolayer-functionalized substrates followed by the post-base treatment and found that that the rate of micropore formation increases with increasing molecular weight of P2VP. ${ }^{10}$ Upon comparing the assembly of P4VP and P2VP of almost the same molecular weight, micropore formation in P2VP/PAA was found to be faster than in P4VP/PAA. ${ }^{10}$

In Chapter 6, a comparison of the ink reservoir capacity of a bare silicon nitride tip and a LbL15 multilayer-coated AFM tip (15 denotes the number of P4VP/PAA bilayers) was presented. Here in this chapter, we first describe the characterization of porous tips prepared under different conditions while varying parameters including molecular weight of P4VP, number of P4VP/PAA bilayers and duration of $\mathrm{KOH}$ solution treatment. The porous cantilevers were all characterized by scanning electron microscopy (SEM) and atomic force microscopy (AFM). It is interesting to investigate how these parameters result in differences in the functionality of the porous structure as an ink reservoir. Tailoring these parameters enables one to select the best protocol to prepare porous tips for performing long-term dip-pen nanolithography (DPN) experiments. The results obtained may also be applied to porous multilayer-coated poly(dimethylsiloxane) (PDMS) stamps for multiple microcontact printing $(\mu \mathrm{CP}) .{ }^{11}$ To study the ink reservoir properties of the porous films prepared under different conditions, two kinds of ink molecules, histidine-tagged enhanced green fluorescent protein (His-EGFP, $27 \mathrm{kDa}$ ) and histidine-tagged tetrameric reef coral fluorescent protein (His-DsRed, $\sim 100 \mathrm{kDa}$ ), were used. 


\subsection{Results and Discussion}

\subsubsection{Fabrication of porous multilayer-coated AFM tips}

The procedure for preparing multilayer-coated porous AFM tips was in principle the same as that illustrated in Scheme 6.1. Briefly, amino-terminated monolayer ( $N$-[3-(trimethoxysilyl) propyl]-ethylenediamine)-functionalized silicon nitride cantilevers were alternately incubated into P4VP $(1 \mathrm{mg} / \mathrm{ml})$ and PAA $(1 \mathrm{mg} / \mathrm{ml})$ methanol solutions to assemble LbL films. These LbL film-coated cantilevers were subsequently immersed into a $\mathrm{KOH}$ solution to form porous structures. Table 7.1 summarizes the different conditions that were applied to prepare a range of porous tips, including different molecular weight (MW) of P4VP (50,000 and 160,000 Da), number of bilayers (15 and 25) and duration of $\mathrm{KOH}(0.1 \mathrm{~N})$ solution treatment $(15,30,45$ and 60 minutes $)$.

Table 7.1. Summary of porous tips prepared at different conditions. Three parameters were utilized to tune the variations of porous morphology, including molecular weight of $\mathrm{P} 4 \mathrm{VP}$, number of bilayers and duration of $\mathrm{KOH}$ solution treatment.

\begin{tabular}{|c|c|c|c|}
\hline & Molecular weight of $\mathrm{P} 4 \mathrm{VP}$ & Number of bilayers & Duration of KOH $(0.1 \mathrm{~N})$ treatment \\
\hline \multirow{16}{*}{ Porous tips } & \multirow{8}{*}{ P4VP $(50,000)$} & \multirow{4}{*}{15} & $15 \min$ \\
\hline & & & $30 \mathrm{~min}$ \\
\hline & & & $45 \mathrm{~min}$ \\
\hline & & & $60 \mathrm{~min}$ \\
\hline & & \multirow{4}{*}{25} & $15 \mathrm{~min}$ \\
\hline & & & $30 \mathrm{~min}$ \\
\hline & & & $45 \mathrm{~min}$ \\
\hline & & & $60 \mathrm{~min}$ \\
\hline & \multirow{8}{*}{ P4VP $(160,000)$} & \multirow{4}{*}{15} & $15 \mathrm{~min}$ \\
\hline & & & $30 \mathrm{~min}$ \\
\hline & & & $45 \mathrm{~min}$ \\
\hline & & & $60 \mathrm{~min}$ \\
\hline & & \multirow{4}{*}{25} & $15 \mathrm{~min}$ \\
\hline & & & $30 \mathrm{~min}$ \\
\hline & & & $45 \mathrm{~min}$ \\
\hline & & & $60 \mathrm{~min}$ \\
\hline
\end{tabular}


In Chapter 6, the porous tips were prepared one by one, which is not an efficient procedure and which may result in differences of porous morphology between tips. To minimize any variations between individual porous tips, the preparation procedure was modified to ensure the tips were fabricated under the same conditions and that the tip surfaces were functionalized in a parallel fashion simultaneously. Instead of a single cantilever, an array of AFM cantilevers cut from a wafer (380 tips) of silicon nitride probes (Veeco, NP) was used. (Figure 7.1)

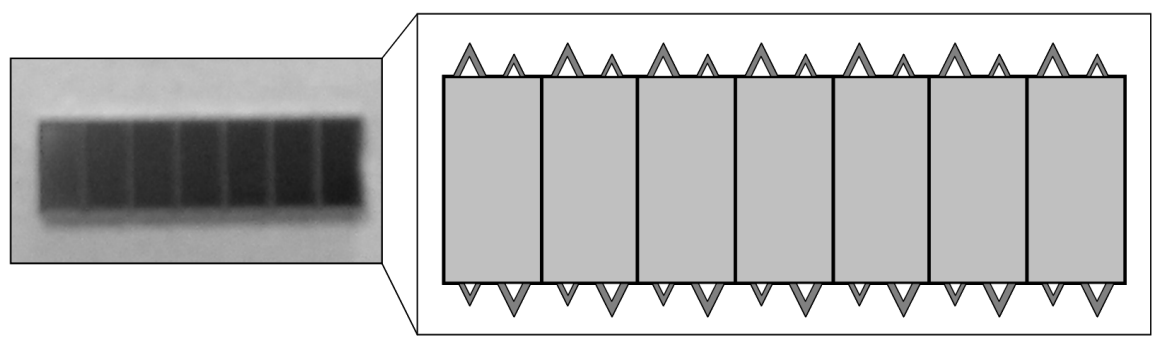

Figure 7.1. Photograph and schematic illustration of an array of $\mathrm{AFM} \mathrm{Si}_{3} \mathrm{~N}_{4}$ cantilevers (Veeco, NP) used for preparing porous multilayer-coated tips.

The morphological variations of the P4VP50000/PAA or P4VP160000/PAA (50000 and 160000 denote the molecular weight of P4VP) LbL films-coated AFM cantilevers upon immersion in the alkaline solution were examined using SEM and AFM. The SEM images of the porous tips fabricated under varied conditions are shown in Figure 7.2a-d. The corresponding supporting chip areas of the cantilevers were examined by AFM in contact mode, and are shown in Figure 7.3. 

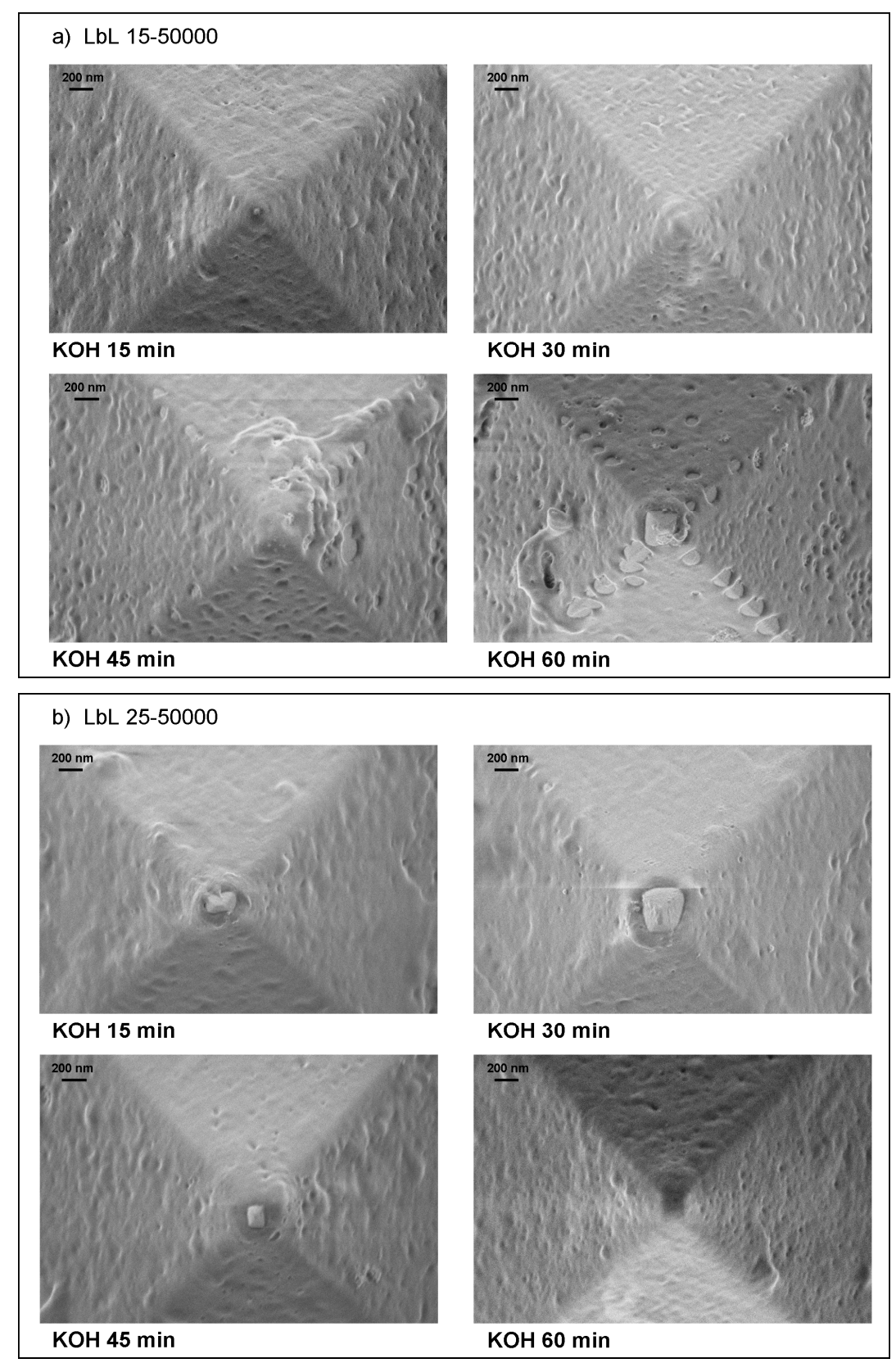

Figure 7.2. SEM images of LbL multilayer-coated porous tips prepared under the conditions of (a) 15 bilayers of P4VP 50000/PAA, (b) 25 bilayers P4VP 50000/PAA, (c) 15 bilayers of P4VP 160000/PAA, (d) 25 bilayers P4VP 160000/PAA with four different durations of $\mathrm{KOH}$ solution (0.1N) treatment varying from 15 minutes to 60 minutes. 


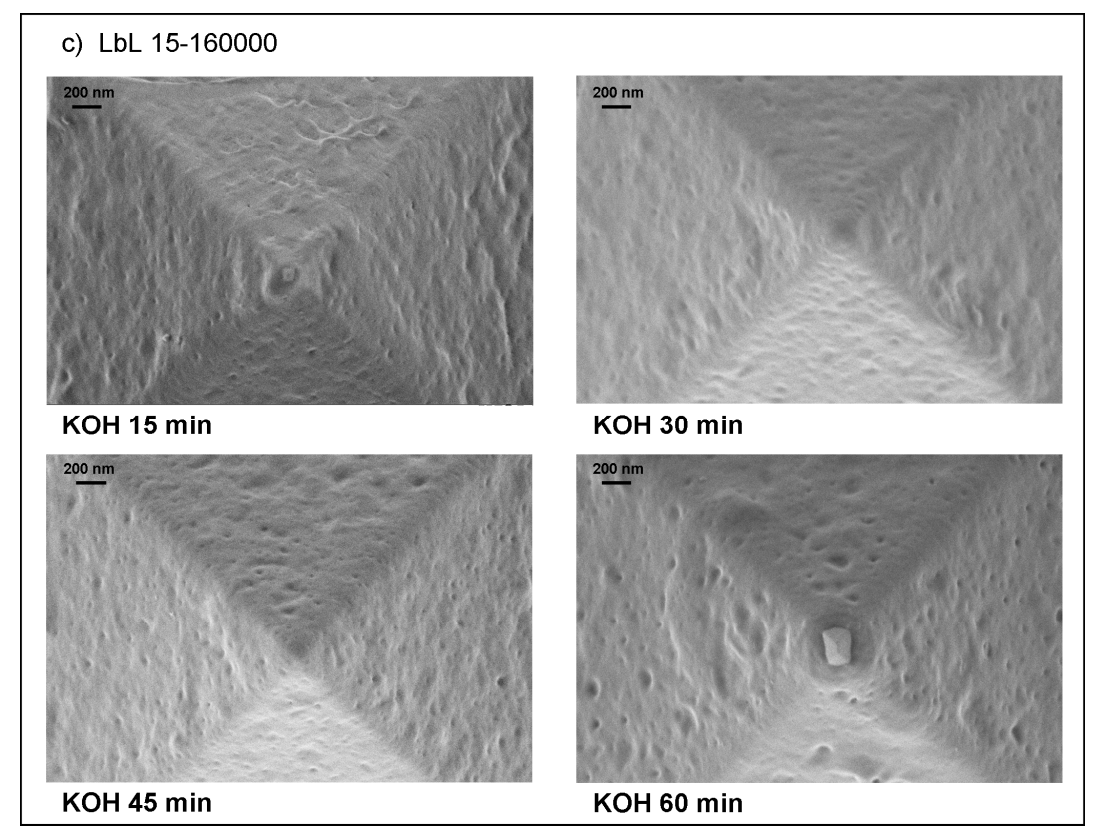

d) LbL $25-160000$

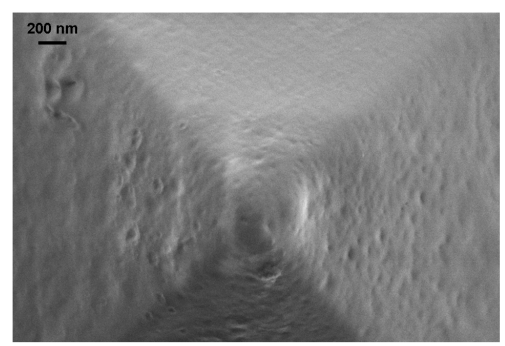

$\mathrm{KOH} 15 \mathrm{~min}$

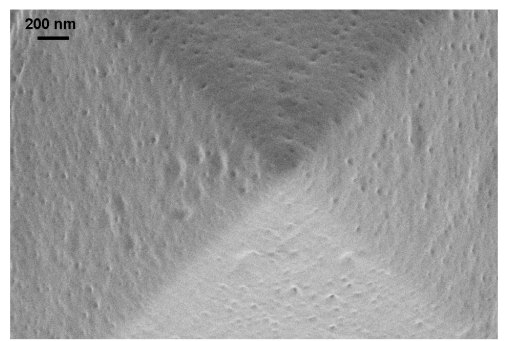

$\mathrm{KOH} 45 \mathrm{~min}$

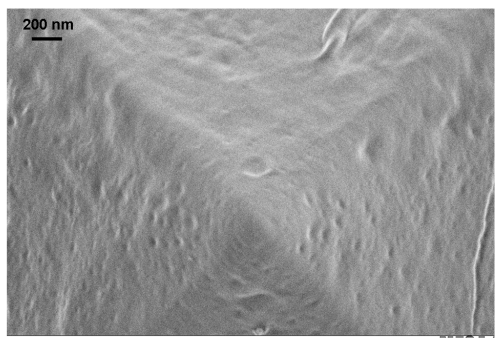

$\mathrm{KOH} 30 \mathrm{~min}$

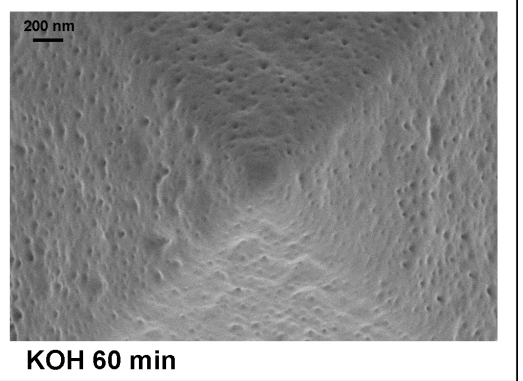

Figure 7.2. SEM images of LbL multilayer-coated porous tips prepared under the conditions of (a) 15 bilayers of P4VP 50000/PAA, (b) 25 bilayers P4VP 50000/PAA, (c) 15 bilayers of P4VP 160000/PAA, (d) 25 bilayers P4VP 160000/PAA with four different durations of $\mathrm{KOH}$ solution $(0.1 \mathrm{~N})$ treatment varying from 15 minutes to 60 minutes. 


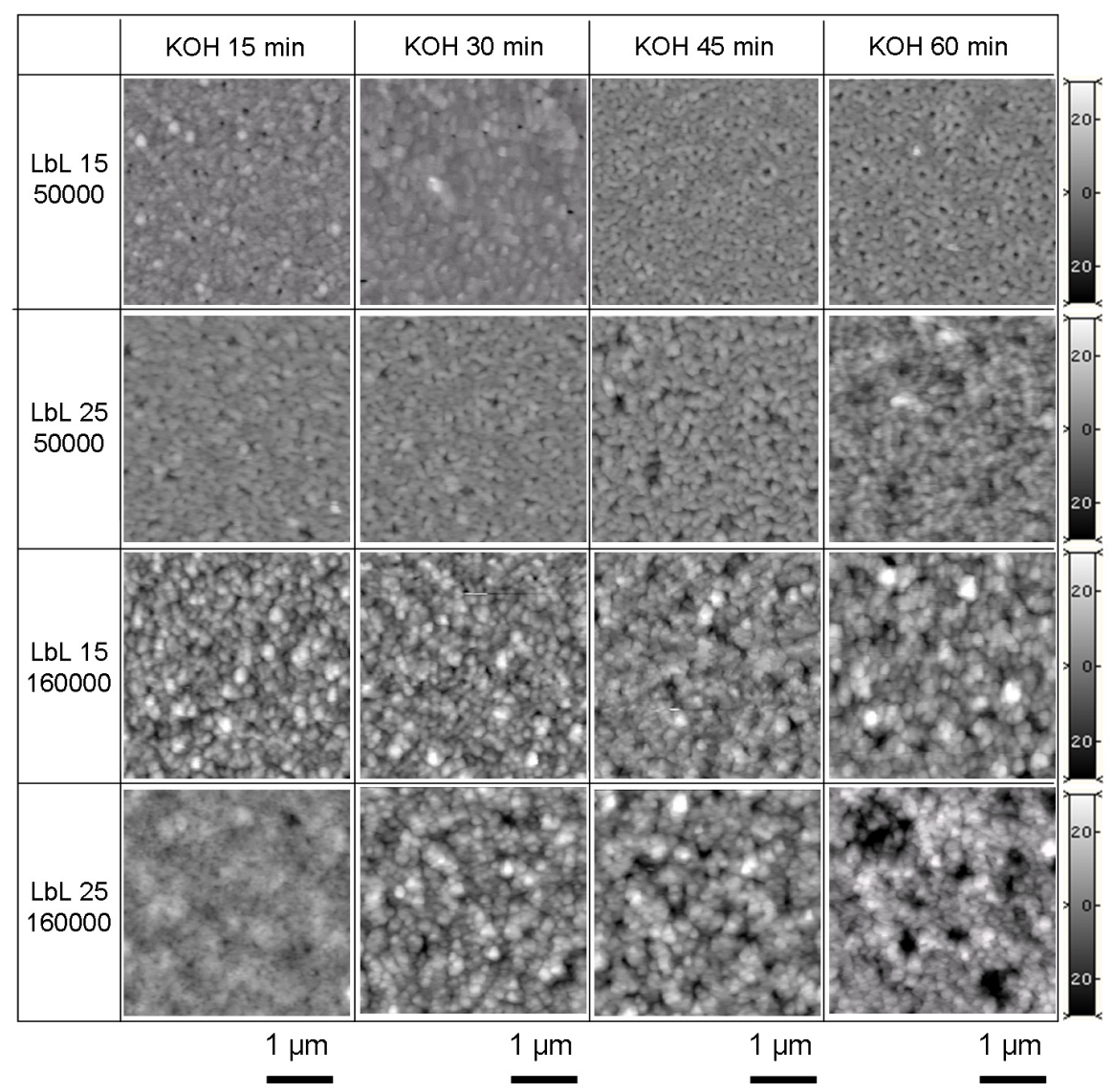

Figure 7.3. Contact mode AFM height images of the LbL-functionalized porous cantilever prepared under various conditions.

The morphologies of the LbL multilayers prepared under different conditions are not the same, but the porous films homogeneously cover the tip and chip surfaces of the cantilevers as shown in Figures 7.2 and 7.3. From both SEM and AFM results, we concluded that the molecular weight of P4VP and the duration of post-base treatments are both important parameters in determining surface morphologies. Except for the porous films prepared with 25 bilayers P4VP 160000/PAA, there are no apparent changes of porous structures after 15 or 30 minutes treatment with $\mathrm{KOH}$ solution. The morphology of LbL multilayers is apparently rather insensitive to the duration of post-base treatment, which allows us to prepare porous tips with certain tolerance. Nevertheless, larger and 
deeper holes were obtained by treating LbL multilayers with the base solution for longer times, which enables us to tune the pore sizes. Compared with SEM images, AFM images (Figure 7.3) exhibit more obvious differences between porous films using P4VP 50000 and P4VP 160000. By using P4VP with higher molecular weight, larger grainy structures of re-conformed P4VP are obtained. In contrast, smoother porous morphology can be obtained by using P4VP with lower molecular weight. All the prepared porous multilayer-coated silicon nitride tips described above were used to study the ink loading properties.

\subsubsection{Ink loading study of porous multilayer-coated AFM tips}

Histidine-tagged enhanced green fluorescent protein (His-EGFP, $27 \mathrm{kDa}$ ) and histidine tagged-tetrameric reef coral fluorescent protein (His-DsRed, $\sim 100 \mathrm{kDa}$ ) were used as ink molecules to study the ink reservoir properties of the porous multilayers. Tris-HCl buffer (10 mM, pH 7.5) containing $1 \mu \mathrm{M}$ His-EGFP or His-DsRed solution was applied onto each multilayer-coated porous cantilever, as well as on a $\mathrm{N}$-[3-(trimethoxysilyl)propyl]-ethylenediamine (TPEDA) monolayer-functionalized cantilever and a bare $\mathrm{Si}_{3} \mathrm{~N}_{4}$ cantilever for 10 minutes. After the loading of ink, the drops of protein solution were removed with a pipette; the cantilevers were rinsed with few drops of Milli-Q water, and dried carefully under a stream of $\mathrm{N}_{2}$ before imaging with a fluorescence microscope. The intrinsic fluorescence of the visible fluorescent proteins enables facile quantification of the amounts of fluorescent proteins encapsulated into the porous films by measuring the fluorescence intensities of the protein-infiltrated functionalized tips.

The exposure time for all the acquired fluorescence images of cantilevers inked with either His-EGFP or His-DsRed was fixed at $600 \mathrm{~ms}$. In order to compare the ink reservoir properties of different porous films, the average fluorescence intensities over 10 different areas with a size of $90 \times 90$ pixels $\left(15 \times 15 \mu^{2}\right)$ from each fluorescence image obtained were first corrected for the background signal and then averaged. Figures $7.4 \mathrm{a}$ and $7.5 \mathrm{a}$ are bar charts of the average fluorescence intensities for the His-EGFP and His-DsRed loaded porous multilayers-coated silicon nitride cantilevers prepared under different conditions respectively. One representative image of the ten fluorescence images of His-EGFP and His-DsRed acquired at each condition are shown in Figures 7.4b and 7.5b, respectively. 
a)
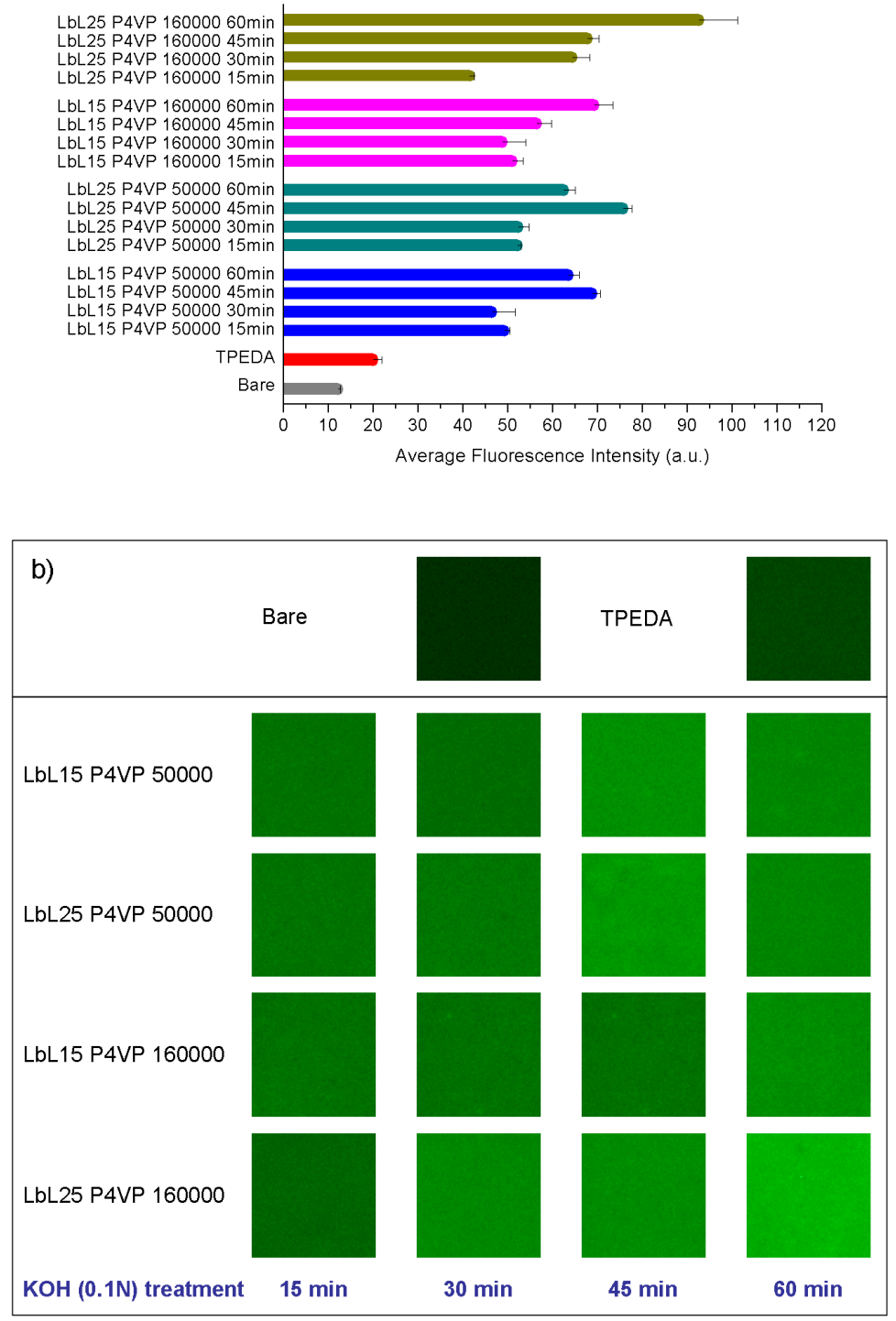

Figure 7.4. (a) Bar charts of average fluorescence intensities of the His-EGFP loaded porous multilayers-coated silicon nitride cantilevers prepared under different conditions. Error bars indicate standard deviation. (b) Representative fluorescence images of His-EGFP loaded porous multilayers-coated silicon nitride cantilevers prepared under different conditions. The image size is $15 \times 15 \mu \mathrm{m}^{2}$ and the color scale of each image is the same. 
a)
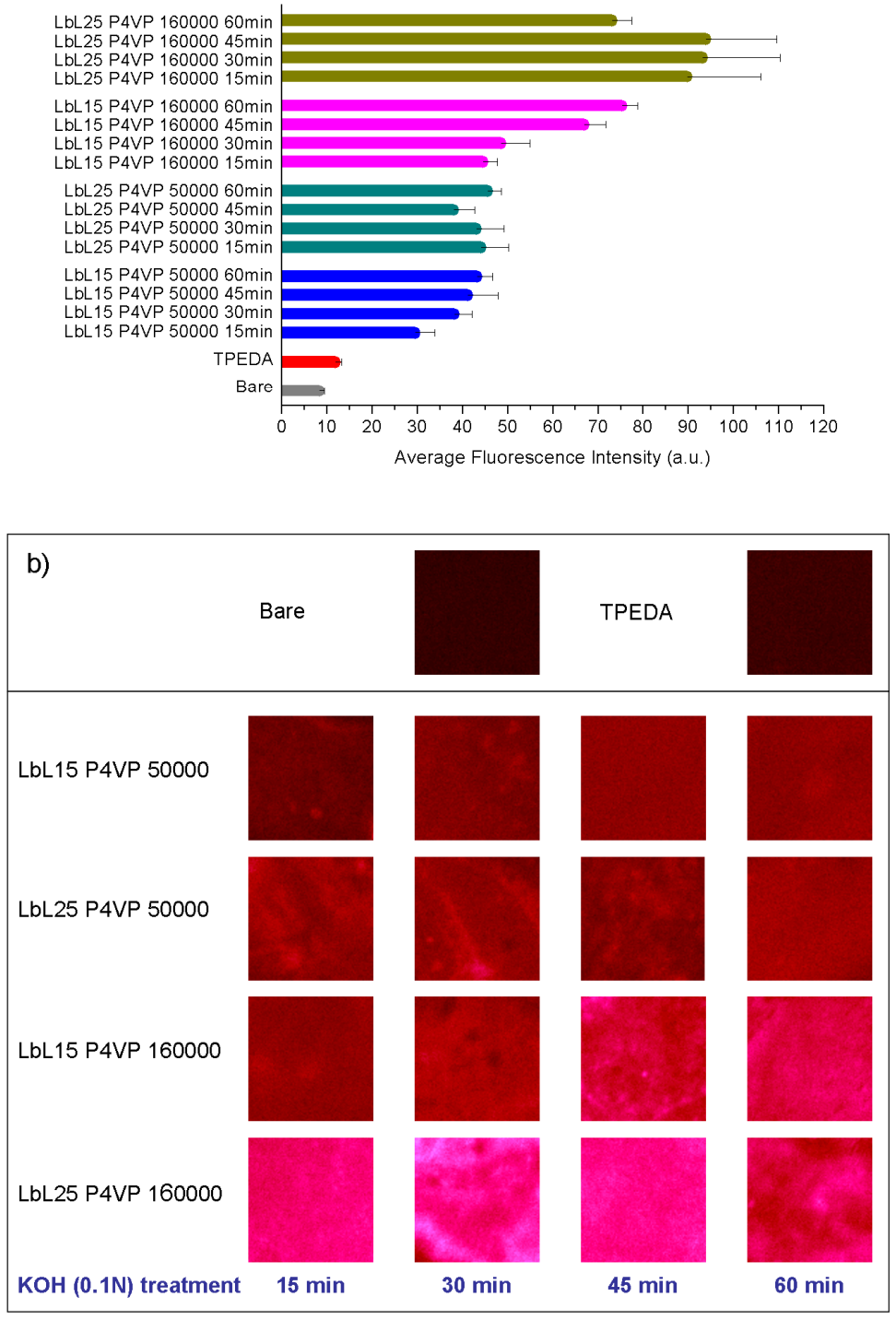

Figure 7.5. (a) Bar charts of the average fluorescence intensities of the His-DsRed loaded porous multilayers-coated silicon nitride cantilevers prepared under different conditions. Error bars indicate standard deviation. (b) Representative fluorescence images of His-DsRed loaded porous multilayers-coated silicon nitride cantilevers prepared under different conditions. The image size is $15 \times 15 \mu^{2}$ and the color scale of each image is the same. 
From Figures 7.4 and 7.5, it is clear that when using P4VP with lower molecular weight (P4VP 50000), the number of assembling P4VP/PAA bilayers does not strongly influence the average fluorescence intensities of His-EGFP or His-DsRed. Compared with His-EGFP, His-DsRed has a greater tendency to adhere to the porous film formed by the remaining P4VP with higher molecular weight (P4VP 160000). This result suggests that in order to encapsulate more His-DsRed, P4VP 160000 should be used for assembling bilayers onto the surfaces of the cantilever. Therefore, the properties of the inks used, e.g. molecular weights and hydrophobicity, also play an important role in optimizing the amount of ink in the porous reservoir.

The changes in the average fluorescence intensities obtained by imaging encapsulated His-EGFP generally correspond to the morphology variations of the porous films shown in Figure 7.3. That is, when the porous films with bigger and deeper pores are inked with His-EGFP, higher average fluorescence intensities are observed in general. However, this observation is not obvious while loading His-DsRed into porous films prepared from 25 bilayers of P4VP and PAA.

Figures 7.4 and 7.5 show that tuning all parameters is necessary in order to encapsulate the highest amount of ink molecules and that all the porous cantilevers inked with His-EGFP or His-DsRed have at least more than threefold increase in the fluorescence intensities than those of the bare cantilevers. In addition, inking a LbL25 P4VP 160000 (KOH 60 minutes) porous tip with His-EGFP or LbL25 P4VP 160000 (KOH 45 minutes) with His-DsRed shows $\sim 9$ times higher fluorescence intensities than inking with bare tips. These results successfully demonstrate that more fluorescent proteins can be encapsulated into the porous films, which effectively act as a sponge for long-term DPN writing.

\subsection{Conclusions}

In summary, this chapter deals with a systematic investigation of surface-modified porous AFM tips using the $\mathrm{LbL}$ technique presented in Chapter 6. The morphology variations of porous films prepared upon varying different parameters, including molecular weight of P4VP, numbers of P4VP/PAA bilayers and duration of $\mathrm{KOH}$ treatment have been investigated using SEM and AFM. These porous tips prepared under various conditions can be used for studying the ink reservoir capacities by loading 
His-EGFP and His-DsRed as ink molecules. We observe that larger amounts of His-EGFP can be encapsulated into the porous films with bigger and deeper pores, and that His-DsRed tends to adhere better to the porous films prepared using P4VP with higher molecular weight. The ink reservoir properties of the porous films loaded with various ink molecules behave differently, a phenomenon that is complex and not fully understood. Nevertheless, this chapter demonstrates that tuning all the parameters is an important procedure to select the best protocol to prepare tips that can encapsulate the largest amount of ink molecules for performing long-term DPN experiments.

\subsection{Experimental}

\section{General procedures.}

All glassware used to prepare the monolayers was cleaned by sonicating for 60 minutes in a $2 \% \mathrm{v} / \mathrm{v}$ Hellmanex II solution in high purity water (Milli-Q, $18.2 \mathrm{M} \Omega \mathrm{cm}$ ), and then rinsed thoroughly with Milli-Q water, and dried in an ambient environment. Arrays of $\mathrm{Si}_{3} \mathrm{~N}_{4}$ AFM cantilevers (NP, Veeco) were cleaned in piranha solution for 1 minute, (concentrated $\mathrm{H}_{2} \mathrm{SO}_{4}$ and $33 \%$ aqueous $\mathrm{H}_{2} \mathrm{O}_{2}$ in a 3:1 ratio). [Warning: Piranha solution should be handled with caution: it has been reported to detonate unexpectedly.] The cantilevers were then rinsed several times with Milli-Q water, and dried carefully with $\mathrm{N}_{2}$ before forming the amino-terminated monolayer ( $N$-[3-(trimethoxysilyl)propyl]ethylenediamine, TPEDA). The preparation of TPEDA monolayer on the surfaces of AFM tips was described in detail in Chapter 6.

\section{Fabrication of multilayer-coated porous tip.}

The multilayer-coated AFM tips were prepared by following the procedures described in Chapter $6{ }^{1}$ Poly(acrylic acid) (PAA), m.w. 5000, $50 \mathrm{wt} \%$ solution in water was first freeze-dried to obtain pure PAA. Commercially available Poly (4-vinylpyridine) (P4VP), MW 50,000 and MW 160,000 was used directly without further purifications. P4VP 50000, P4VP 160000 and PAA were dissolved in methanol individually to obtain $1 \mathrm{mg} / \mathrm{ml} \mathrm{P4VP}$ and PAA methanol solutions. The TPEDA monolayer-functionalized AFM cantilevers were first incubated into either P4VP 50000 or P4VP 160000 solution for 10 minutes and then rinsed with methanol several times before being incubated into PAA solution for 10 minutes. This process was repeated for a defined number of times to obtain LbL-functionalized cantilevers. The LbL-functionalized cantilevers were subsequently immersed into $\mathrm{KOH}$ solution of $0.1 \mathrm{~N}$ for various durations to obtain porous structures coated cantilevers. The porous cantilevers were rinsed with Milli-Q water and dried very gently under a stream of $\mathrm{N}_{2}$ before characterizing porous structures and loading ink molecules. 


\section{Analysis of porous structures}

The surface structures of porous cantilevers were characterized by a scanning electron microscope (SEM) (Zeiss) and a custom-built atomic force microscope (AFM) in contact mode equipped with a $\mathrm{Si}_{3} \mathrm{~N}_{4}$ AFM cantilever (MSCT, Veeco) with a nominal spring constant of $0.54 \mathrm{~N} / \mathrm{m}$. The scan speed of all AFM images is 1000 pixels per second with a scan range of $\sim 3400 \mathrm{~nm}$ in width. The obtained AFM height images were analyzed using the commercial software SPIP (Image Metrology, Version 4.4.3.0).

\section{Acquisition of fluorescent images.}

The porous tip and the bare $\mathrm{Si}_{3} \mathrm{~N}_{4}$ tip inked with His-EGFP or His-DsRed were placed onto a cleaned glass substrate and then examined with a fluorescence microscope (TE2000-U, Nikon) equipped with a mercury lamp as light source and a digital camera (Nikon digital Sight DS-Qi1Mc). To obtain fluorescence images of His-EGFP adhered to tip surfaces, the filter cube with blue excitation light $(\lambda=470 / 22 \mathrm{~nm})$, green emission light $(\lambda=520 / 35 \mathrm{~nm})$ and dichroic $(\lambda=495 \mathrm{~nm})$ was used. To obtain fluorescence images of His-DsRed, the filter cube with green excitation light $(\lambda=510 / 40 \mathrm{~nm})$, red emission light $(\lambda=617 / 73 \mathrm{~nm})$ and dichroic $(\lambda=562 \mathrm{~nm})$ was used. The exposure time for all the acquired fluorescence images of cantilevers inked with fluorescent proteins was fixed at $600 \mathrm{~ms}$. The fluorescence images obtained were analyzed using the commercial software SPIP (Image Metrology, Version 4.4.3.0).

\subsection{References}

1. Fu, Y.; Bai, S. L.; Cui, S. X.; Qiu, D. L.; Wang, Z. Q.; Zhang, X. Hydrogen-Bonding-Directed Layer-by-Layer Multilayer Assembly: Reconformation Yielding Microporous Films. Macromolecules 2002, 35, 9451-9458.

2. Wang, L. Y.; Fu, Y.; Wang, Z. Q.; Fan, Y. G.; Zhang, X. Investigation into an Alternating Multilayer Film of Poly(4-Vinylpyridine) and Poly(Acrylic Acid) Based on Hydrogen Bonding. Langmuir 1999, 15, 1360-1363.

3. Wang, L. Y.; Wang, Z. Q.; Zhang, X.; Shen, J. C.; Chi, L. F.; Fuchs, H. A New Approach for the Fabrication of an Alternating Multilayer Film of Poly(4-Vinylpyridine) and Poly(Acrylic Acid) Based on Hydrogen Bonding. Macromol. Rapid Commun. 1997, 18, 509-514.

4. Zhang, X.; Chen, H.; Zhang, H. Y. Layer-by-Layer Assembly: From Conventional to Unconventional Methods. Chem. Commun. 2007, 1395-1405.

5. Wu, C. C.; Xu, H. P.; Otto, C.; Reinhoudt, D. N.; Lammertink, R. G. H.; Huskens, J.; Subramaniam, V.; Velders, A. H. Porous Multilayer-Coated Afm Tips for Dip-Pen Nanolithography of Proteins. J. Am. Chem. Soc. 2009, 131, 7526-7527.

6. Kharlampieva, E.; Kozlovskaya, V.; Sukhishvili, S. A. Layer-by-Layer Hydrogen-Bonded Polymer Films: From Fundamentals to Applications. Adv. Mater. 2009, 21, 3053-3065. 
7. Bai, S. L.; Wang, Z. Q.; Gao, J.; Zhang, X. Hydrogen-Bonding-Directed Layer-by-Layer Polymer Films: Substrate Effect on the Microporous Morphology Variation. Eur. Polym. J. 2006, 42, 900-907.

8. Bai, S. L.; Wang, Z. Q.; Zhang, X.; Wang, B. Hydrogen-Bonding-Directed Layer-by-Layer Films: Effect of Electrostatic Interaction on the Microporous Morphology Variation. Langmuir 2004, 20, 11828-11832.

9. Zhang, H. Y.; Fu, Y.; Wang, D.; Wang, L. Y.; Wang, Z. Q.; Zhang, X. Hydrogen-Bonding-Directed Layer-by-Layer Assembly of Dendrimer and Poly(4-Vinylpyridine) and Micropore Formation by Post-Base Treatment. Langmuir 2003, 19, 8497-8502.

10. Zhang, H. Y.; Wang, D.; Wang, Z. Q.; Zhang, X. Hydrogen Bonded Layer-by-Layer Assembly of Poly(2-Vinylpyridine) and Poly(Acrylic Acid): Influence of Molecular Weight on the Formation of Microporous Film by Post-Base Treatment. Eur. Polym. J. 2007, 43, 2784-2791.

11. Xu, H. P.; Gomez-Casado, A.; Liu, Z. H.; Reinhoudt, D. N.; Lammertink, R. G. H.; Huskens, J. Porous Multilayer-Coated Pdms Stamps for Protein Printing. Langmuir 2009, 25, 13972-13977. 


\section{Appendix}

In addition to using visible fluorescent proteins as ink molecules, amine-containing quantum dots (QDs- $\mathrm{NH}_{2}$, eBioscience, Inc.) were also used to compare the ink reservoir property of the porous multilayer-coated AFM tips. From Figure A7.1, it can be seen that the porous LbL15 tips can encapsulate more $\mathrm{QDs}-\mathrm{NH}_{2}$ than a bare or amino-terminated monolayer (TPEDA monolayer)-functionalized tip.

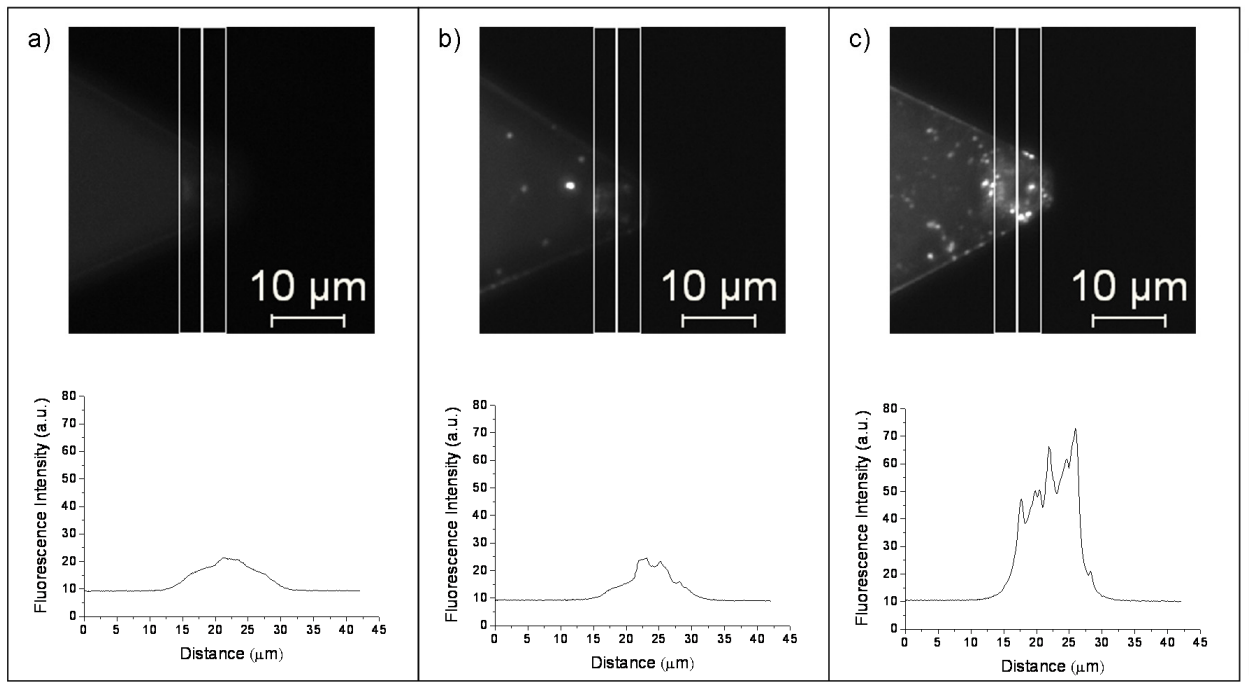

Figure A7.1. Fluorescence images and average fluorescence intensity profiles of the area inside the rectangle of (a) a bare AFM tip, (b) an amino-terminated monolayer (TPEDA monolayer)-functionalized tip, and (c) a porous LbL15 tip, dipped into amine-containing quantum dots $\left(\mathrm{QDs}-\mathrm{NH}_{2}\right)$ solution $(200 \mathrm{nM})$ for 10 minutes and then dried with $\mathrm{N}_{2}$. The exposure time of each image is $400 \mathrm{~ms}$. 


\section{Summary}

Dip-pen nanolithography (DPN) is an atomic force microscopy (AFM)-based lithography technique offering the possibility of fabricating patterns with feature sizes ranging from micrometers to tens of nanometers, utilizing either top-down or bottom-up strategies. Although during its early development stages the serial nature of operation of DPN restricted the patterning efficiency, the successful design and fabrication of AFM probe arrays have increased the throughput of DPN dramatically by enabling generation of patterns in parallel. Several advantages of DPN including (1) ease of generation of arbitrary patterns, (2) application to a variety of ink-substrate combinations, and (3) lower cost relative to other lithographic techniques, have led to many applications in different fields and the establishment of DPN as a popular tool for nanofabrication.

This thesis contributes to the continuing development of the DPN technique, mainly focusing on two aspects. The first aspect, presented in Chapters 3-5, is to use DPN to fabricate metal-ion and (bio)-molecular patterns at micrometer or sub-micrometer scale onto monolayer-functionalized solid surfaces with different chemical immobilization strategies. Before performing all DPN experiments, microcontact printing $(\mu \mathrm{CP})$, a well-known and understood simple and rapid soft lithography technique, was used first to confirm the usefulness of the ink-substrate interactions. The second aspect of interest is to develop an AFM tip surface-modification method for long-term DPN writing, as described in Chapters 6 and 7. A brief synopsis of all chapters follows:

In Chapter 1, a general introduction to this thesis is given.

In Chapter 2 the development of the DPN technique in the past decade and the related chemical strategies are reviewed, classified into indirect DPN and direct DPN, which have been reported to fabricate biomolecular (proteins, peptides, and DNA) architectures onto solid surfaces. In addition, some other AFM-based lithography techniques, such as nanoshaving/nanografting, bias AFM lithography and fountain pen lithography, used for bio-patterning, are also briefly described at the end of this chapter.

In Chapter 3 first a brief introduction of a custom-built instrument, the atomic force fluorescence microscope (AFFM) is given and the simple relative humidity (RH) control 
system is described that was designed for implementation with the AFFM. In this thesis, all DPN experiments were performed with the AFFM, which combines an atomic force microscope and a confocal fluorescence microscope and therefore allows one to write patterns and subsequently visualize the written results in situ in a sequential write-read mode. Subsequently, a modification of the protocol for preparing fluorescent monolayers sensitive to metal ions is described. The controlled delivery of $\mathrm{Ca}$ (II) ions with DPN onto fluorescent sensitive monolayer-functionalized glass substrates is demonstrated by visualizing the enhancement of the fluorescence intensity. The driving force for the transfer of these ions from an AFM tip to the surface is their complexation to the organic ligands on the monolayer.

Metal ions represent the smallest sized ink molecules for DPN. A potential application of metal ion patterns is that they can be utilized as a template to immobilize biomolecules. Chapter 4 demonstrates the possibility of this application by generating $\mathrm{Ni}(\mathrm{II})$ ion patterns at micro- and submicrometer scale onto nitrilotriacetic acid (NTA)-terminated monolayers-functionalized glass substrates with $\mu \mathrm{CP}$ and DPN, respectively. The patterned Ni(II)-NTA monolayers allow a single layer of histidine-tagged visible fluorescent proteins via a specific metal-protein interaction, which was characterized by AFM topography measurements. This is an indirect DPN method of fabricating protein patterns that can prevent denaturation of fragile biomolecules caused by direct printing or writing. We have also demonstrated by $\mu \mathrm{CP}$ that the metal ions complexed with His-tagged proteins can be easily removed by rinsing with an EDTA solution for reusing the functionalized substrates. The property of being able to regenerate and reuse the substrate is potentially useful for the fabrication of multiple protein patterns. Additionally, complexing with His-tagged fluorescent proteins offers the possibility of fundamental parameters of DPN regarding the dependence of transfer of Ni(II) ink ions, such as writing speeds and tip-surface contact times, since the small size of $\mathrm{Ni}$ (II) ions is practically extremely difficult to characterize directly.

In Chapter 3 and Chapter 4, non-covalent interactions have been described to generate patterns. In Chapter 5, a reactive $\mu \mathrm{CP}$ and $\mathrm{DPN}$ approach is presented for immobilizing amine containing $\beta$-cyclodextrin $\left(\beta C D-\mathrm{NH}_{2}\right)$ and quantum dots $\left(\mathrm{QD}-\mathrm{NH}_{2}\right)$ onto $N, N$-carbonyldiimidazole-activated amino monolayer (IM monolayer) at micrometer scale via the covalent formation of urethane bonds. To confirm the covalent formation of $\beta C D-\mathrm{NH}_{2}$ patterns, adamantyl-labeled fluorophores or ferrocenyl-labeled fluorescent proteins were used to image the patterns obtained. This is the first demonstration of using 
DPN to covalently anchor molecules onto IM monolayers. In addition, orthogonal microcontact printing of $\beta C D-\mathrm{NH}_{2}$ and $\mathrm{QD}-\mathrm{NH}_{2}$ patterns is succesfully demonstrated, suggesting a viable chemical approach for fabricating patterns of different functional groups through reactive DPN for selective immobilization of multiple desired molecules with complementary functionalities to be available in the near future.

One of the limitations of DPN is ink depletion from the tip, which limits the amount of ink molecules that can be written. In Chapter 6, a rather simple method is presented to coat the surfaces of AFM tips with a thin layer of porous film by using Layer-by-Layer (LbL) techniques. The pore sizes can be adjusted by treating the poly4-(vinylpyridine)/poly(acrylic acid) (P4VP/PAA) films coated onto amino-terminated monolayer $\left(\mathrm{NH}_{2}\right.$-monolayer)-functionalized AFM tip surfaces with a base solution for different periods of time. This hydrophilic porous film can easily absorb biomolecules and act as a larger volume ink reservoir compared to a bare AFM tip. Proof-of-concept of the porous AFM tip is demonstrated by using fluorescent proteins as ink molecules to fabricate protein patterns at micrometer and sub-micrometer length scales.

A further investigation of the morphology variations of porous films-coated silicon nitride AFM tips, prepared under various conditions, is described in Chapter 7. The variable parameters include the molecular weight of poly(4-vinylpyridine) (P4VP), the number of assembled layers, and duration of base solution treatment. These porous tips prepared under different conditions have been investigated for their ink reservoir properties by loading two different kinds of proteins, histidine-tagged enhanced green fluorescent protein (His-EGFP) and histidine-tagged tetrameric reef coral fluorescent protein (His-DsRed), as ink molecules. We find that, as expected, larger amounts of His-EGFP can be encapsulated into the porous films with bigger and deeper pores, and that His-DsRed tends to adhere to the porous films prepared using P4VP with higher molecular weight. This chapter demonstrates that one can tune all the parameters in order to select the best protocol to prepare tips that can encapsulate the largest amount of ink molecules for performing long-term DPN experiments.

Collectively, the results presented in this thesis illustrate a range of new ink-substrate combinations that can be applied with DPN to create patterns at micro- and sub-micrometer scale. The fabrication of Ni(II) ion patterns onto NTA-terminated monolayers via coordinating interactions is a general indirect DPN approach to immobilize a monolayer of His-tagged proteins, with the advantage of avoiding denaturation of fragile biomolecules readily observed when printing or writing proteins 
directly. The preliminary demonstration of reactive DPN by utilizing the quick covalent formation of urethane bonds between imidazolide and amine groups may become a potential chemical strategy to generate patterns of various functional groups for selective immobilization of multiple desired molecules with complementary functionalities. Surface modification of AFM tips with a hydrophilic porous film prepared by LbL technique enhances the loading capacities of the tip, demonstrated using biomolecules and amine-containing quantum dots. The simplicity and ease of adjusting the pore sizes enable us to optimize the porosity for encapsulating the maximum amount of specific ink molecules. Therefore, the multilayer-coated porous tips show the potential to be a universal approach for long-term production of (bio)molecular patterns onto surfaces at hundreds of nanometers scale. 


\section{Samenvatting}

Dip-pen nanolithografie (DPN) is een lithografietechniek gebaseerd op atomaire kracht microscopie (atomic force microscopy, AFM) die de mogelijkheid levert voor het maken van patronen in de ordegrootte van micrometers tot tientallen nanometers, middels een top-down of bottom-up strategie. Alhoewel de efficiëntie van het maken van de patronen in het begin van de ontwikkeling werd belemmerd door de seriële aard van de DPN operaties, heeft het ontstaan van AFM tip array, waarmee patronen in parallel gemaakt kunnen worden, de snelheid van DPN aanzienlijk verbeterd. De voordelen van DPN zijn, onder andere, (1) het gemak van het genereren van willekeurige patronen, (2) veel inkt-substraat combinaties zijn mogelijk, en (3) kost minder dan andere lithographietechnieken. Deze voordelen hebben voor veel applicaties van DPN in verschillende velden gezorgd en DPN tot een populaire methode voor nanofabricage gemaakt.

Dit proefschrift levert een bijdrage aan de verdere ontwikkelingen van de DPN techniek, gericht op twee aspecten. Het eerste aspect, geïntroduceerd in hoofstukken 3-5, betreft het gebruik maken van DPN voor het genereren van metaal-ion en (bio)-moleculaire patronen op micrometer- of submicrometerschaal op monolaag-bewerkte oppervlakken met verschillende chemische immobilisatie strategieën. Vóór het uitvoeren van alle DPN experimenten, was eerst microcontact-drukken $(\mu \mathrm{CP})$, een bekende en goed begrepen simpele en snelle zacht-lithografische techniek, gebruikt om de geschiktheid van de inkt-substraat interacties te bevestigen. Het tweede aspect betreft het ontwikkelen van een oppervlak-modificatie methode die langdurig DPN schrijven mogelijk maakt, zoals beschreven in hoofdstukken 6 en 7. Een kleine samenvatting van de afzonderlijke hoofdstukken is als volgt:

In hoofdstuk 1 wordt een algemene inleiding tot dit proefschrift gegeven.

In hoofdstuk 2 worden de ontwikkeling van DPN techniek in het afgelopen decennium en gerelateerde chemische strategiën behandeld, geclassificeerd als indirect DPN en direct DPN, die bekend zijn vanwege het fabriceren van biomoleculaire (eiwitten, peptides, en DNA) strukturen op oppervlakken. Bovendien zijn andere 
lithografietechnieken die op AFM zijn gebasseerd en die gebruikt worden om bio-patronen te maken, zoals nanoscheren/nanoschuren bias AFM lithografie en vulpen lithografie, ook kort beschreven aan het einde van dit hoofdstuk.

In hoofdstuk 3 wordt eerst een zelfgemaakt instrument, de atomic force fluorescence microscope (AFFM), geintroduceerd en ook een beschrijving van een simpel relatieve vochtigheid controlesysteem dat ontworpen is om met de AFFM te kunnen samenwerken. In dit proefschrift zijn alle DPN experimenten uitgevoerd met de AFFM, die een combinatie is van een atomair kracht microscope en een confocaal fluorescentie microscoop. Deze combinatie geeft de mogelijkheid om na het schrijven van een patroon meteen in situ het schrijfresultaat te kunnen observeren in een sequentiële schrijf-lees modus. Vervolgens is een modificatie van het protocol beschreven voor het voorbereiden van fluorescerende monolagen die gevoelig zijn voor metaalionen. De gecontroleerde afgave van $\mathrm{Ca}(\mathrm{II})$ ionen met DPN op fluorescerende gevoelige monolaag-bewerkte glassubstraten is aangetoond door het visualiseren van de versterking van de fluorescentie-intensiteit. De drijfkracht voor het overdragen van de ionen van een AFM tip naar de oppervlakte is hun complexatie met de organische liganden op de monolaag.

Metaalionen vertegenwoordigen de kleinst mogelijke inktmoleculen voor DPN. Een potentiële toepassing van metaalionen-patronen is dat ze gebruikt kunnen worden als een template om biomoleculen te immobiliseren. Hoofdstuk 4 laat de mogelijkheid van deze toepassing zien door het genereren van $\mathrm{Ni}(\mathrm{II})$ ion patronen op micro- en submicrometer schaal op nitrilotriazijnzuur (NTA)-eindigende monolaag-bewerkte glassubstraten met, respectievelijk, $\mu \mathrm{CP}$ en DPN. De volgens patroon bewerkte monolagen laten een enkele laag van met histidine gelabelde zichtbare fluorescerende eiwitten toe via een specifieke metaal-eiwit interactie, die gekarakteriseerd is door AFM topografiemetingen. Dit is een indirecte DPN methode van eiwitpatronen-fabricage die denaturatie van zwakke biomoleculen veroorzaakt door direct printen of schrijven kan voorkomen. We hebben ook middels $\mu \mathrm{CP}$ laten zien dat de metaalionen complexeerd met His-gelabelde eiwitten gemakkelijk verwijderd kunnen worden door ze af te spoelen met een EDTA oplossing voor het herbruiken van de bewerkte substraten. De eigenschap dat de substraten hergegenereerd en hergebruikt kunnen worden is handig voor het fabriceren van meerdere eiwitpatronen. Bovendien geeft het complexeren met fluorescerende eiwitten gelabeled met His de mogelijkheid van bestuderen van fundamentele parameters van DPN betreffende de afhankelijkheid van $\mathrm{Ni}(\mathrm{II})$ ionen transportatie, zoals schrijfsnelheid en 
tip-oppervlakte aanrakingstijd. De Ni(II) ionen zijn namelijk heel moeilijk direct te karakteriseren door hun kleine formaat.

In hoofdstuk 3 en hoofdstuk 4 zijn niet-covalente interacties beschreven om patronen te genereren. In hoofdstuk 5 wordt een reactieve $\mu \mathrm{CP}$ and DPN aanpak gepresenteerd voor de immobilisatie van amine-gefunctionaliseerde $\beta$-cyclodextrin $\left(\beta \mathrm{CD}-\mathrm{NH}_{2}\right)$ en quantum dots $\left(\mathrm{QD}-\mathrm{NH}_{2}\right.$ ) op $\mathrm{N}, \mathrm{N}$-carbonyldiimidazole-geactiveerde amino monolagen (IM monolagen) op micrometer schaal, via de covalente formatie van urethaan-bindingen. Om de covalente formatie van $\beta C D-\mathrm{NH}_{2}$ vast te stellen zijn fluoroforen met adamantyl of fluorescerende eiwitten met ferrocenyl gebruikt om de gekregen patronen af te beelden. Dit is de eerste demonstratie van het gebruiken van DPN om moleculen op IM monolayers te verankeren op een covalente manier. Additioneel is orthogonale microcontact printing van $\beta C D-\mathrm{NH}_{2}$ and $\mathrm{QD}-\mathrm{NH}_{2}$ met succes gedemonstreerd. Dit suggereert een toepasbare chemische aanpak voor het fabriceren van patronen van verschillende functionele groepen door reactieve DPN voor selectieve immobilisatie van meerdere gewenste moleculen met complementaire functionaliteiten die in de nabije toekomst verkrijgbaar zal zijn.

Een van de limitaties van DPN is het opraken van inkt op de tip, waardoor de hoeveelheid inktmoleculen die gebruikt kunnen worden voor het schrijven wordt beperkt. In hoofdstuk 6 wordt een simpele methode gepresenteerd om op de oppervlaktes van AFM tippen met een dunne laag poreuze film te bekleden door middel van laag-op-laag (Layer-by-Layer, LbL) technieken. De formaten van de poriën kunnen ingesteld worden door de met poly4-(vinylpyridine)/poly(acrylzuur) (P4VP/PAA) films beklede amino-monolaag (NH2-monolaag)-behandelde AFM tip oppervlakken met een basische oplossing te behandelen voor verschillende tijdsspannes. Deze hydrofiele film met poriën kan gemakkelijk biomoleculen absorberen en fungeren als een inktreservoir met grotere inhoud vergeleken met een kale AFM tip. Proof-of-concept van de poreuze AFM tip is gedemonstreerd door lichtgevende eiwitten te gebruiken als inktmoleculen om eiwitpatronen te fabriceren op micrometer en sub-micrometer schaal.

Een nader onderzoek van de morphologische variaties van silicon-nitride AFM tips bekleed met poreuze films, gemaakt onder verschillende condities, wordt beschreven in hoofdstuk 7. De variabele parameters betreffen het moleculaire gewicht van poly(4-vinylpyridine) (P4VP), het aantal gemonteerde lagen, en de tijdsduur van de behandeling met de basisoplossing. Deze poreuze tippen, voorbereid onder verschillende condities, zijn onderzocht voor hun inktreservoir eigenschappen door het laden van twee 
verschillende soorten eiwitten, histidine-gemerkte versterkte groen lichtgevende eiwitten (His-EGFP) en histidine-gemerkte tetrameric koraalrif lichtgevende eiwitten (His-DsRed), als inktmoleculen. Zoals verwacht kan een grotere hoeveelheid His-EGFP ingekapseld worden in de poreuze films met grotere en diepere poriën, en heeft His-DsRed de neiging om aan poreuze films te plakken die voorbehandeld zijn met P4VP met een hoger molecuulgewicht. Dit hoofdstuk toont aan dat men verschillende combinaties van parameters kan uitproberen om het beste protocol te kiezen voor het voorbereiden van tippen zodat die de grootste hoeveelheid inktmoleculen kunnen opnemen voor lange-termijn DPN experimenten.

De resultaten gepresenteerd in dit proefschrift illustreren een reeks van nieuwe inkt-substraat combinaties die gebruikt kunnen worden met DPN om patronen te produceren op micrometer en sub-micrometer schaal. Het fabriceren van Ni(II)-ion patronen op NTA-eindigende monolagen via gecoördineerde interacties is een algemene indirecte DPN aanpak om een monolaag van His-gemerkte eiwitten vast te zetten, met het voordeel dat denaturatie van zwakke biomoleculen, vaak geobserveerd tijdens direct printen of schrijven van eiwitten, wordt vermeden. De voorafgaande demonstratie van reactieve DPN bij het gebruiken van de snelle covalente formatie van urethaanbindingen tussen imidazolide en amine groepen kan een potentiële chemische strategie zijn om patronen van verschillende functionele groepen voor selectieve vastlegging van meerdere moleculen met aanvullende functionaliteiten te genereren. Modificatie van de oppervlakken van de AFM tippen met een hydrofiele poreuze film die voorbereid is met LbL techniek versterkt de laadcapaciteit van de tip, gedemonstreerd door het gebruiken van biomoleculen en amine-bevattende quantum dots. Het gemak waarmee de poriëngroottes aangepast kunnen worden staat ons toe om de poreusheid voor het opnemen van de maximale hoeveelheid specifieke inktmoleculen te optimaliseren. Daardoor tonen de multilagen-beklede poreuze tippen de potentie om een universele aanpak te worden voor het produceren van lange-termijn (bio)moleculen patronen op oppervlakten op een schaal van honderden nanometers. 


\section{Abbreviations}

AFM: Atomic Force Microscope

AFFM: Atomic Force Fluorescence Microscope

$\beta C D: \beta$-cyclodextrin

CDI: $N, N$-carbonyldiimidazole

DPN: Dip-Pen Nanolithography

DsRed: Tetrameric Reef Coral Fluorescent Protein

EGFP: Enhanced Green Fluorescent Protein

(E)SEM: (Environmental) Scanning Electro Microscope

His 6 : Hexahistidine

IM: Imidazolide

LbL: Layer-by-Layer

HCP: Microcontact Printing

MHA: 16-Mercaptohexadecanoic Acid

Ni-NTA: Nickel(II)-Nitrilotriacetic Acid

ODT: 1-Octadecanethiol

QDs: Quantum Dots

OTS: Octadecyltrichlorosilane

P4VP: Poly(4-vinylpyridine)

PAA: Poly(acrylic acid)

PDMS: Poly(dimethylsiloxane)

RH: Relative Humidity

SAMs: Self-Assembled Monoalyers

SPL: Scanning Probe Lithography

TAMRA: 5-(and-6)-Carboxytetramethylrhodamine, succinimidyl ester

THF: Tetrahydrofuran

TPEDA: $N$-[3-(trimethoxysilyl)propyl]ethylenediamine

VFP: Visible Fluorescent Protein 


\section{Acknowledgements}

Some would think that, after wring a whole thesis, the acknowledgement part should be relatively easy. However, it is quite hard for two reasons: to completely cover all the people I would like to thank and to completely express my gratitude to the people mentioned. Still I'm giving it my best try here and hope the words could reach you all.

First I would like to thank my promotors, Prof. David Reinhoudt and Prof. Vinod Subramaniam. It was an honor to work with you and I have learned so much from you, both as being a scientist and as a person. I also want to thank you for your support and trust in me during the whole process that inspired me in achieving more than I ever can imagine. Vinod, when I was feeling down due to lost and worries, thank you for being there to encourage me and help me stand up on my feet again. David, I have always enjoyed your presence and guidance.

I would like to thank Dr. Aldrik Velders and Dr. Cees Otto, my daily supervisors, for pointing me to the right direction during my research and providing support at each step along the way. Aldrik, thank you for providing nice ideas, pushing me to try out different chemistry possibilities and encouraging me all the time. Cees, I really learned a lot from our discussions and regular meetings.

I also would like to thank my former daily supervisor at beginning of my $\mathrm{PhD}$, Dr. Mercedes Crego-Calama who gave me the chance to start my $\mathrm{PhD}$ project. In addition, I would like to thank Dr. Martin Bennink for your supervision and also the programming enabling AFM to generate patterns, which is invaluable to my work.

Special thanks to David, Aldrik and Vinod for giving me such a nice opportunity and lots of support to go to Prof. Mirkin's group in Northwestern University. It really meant a lot to me to see the birthplace of dip-pen nanolithography and learn the practical experiences from them. In addition, although this thesis does not include the work performed in Northwestern University (NU), I would like to take this chance to thank Prof. Chad Mirkin and all the sub-DPN group members in NU for the valuable experience and help.

Furthermore, I would like to thank NanoNed for the financial support and also all the NanoNed staff and user committee members of the Nanofabrication Flagship. 
I would like to express my gratitude to colleagues and friends in BPE, SMCT, and MnF. Because of your contributions, supports and accompanying, I had great time not just in the work part but also in the private life during all these years in Enschede.

Technicians are the first persons I ran to when I encountered difficulties in handling equipments and needed practical support. Kees, thanks for the AFM training and keeping the AFM alive until now. I enjoyed discussing with you on design of the humidity chamber and all the AFM experiments a lot. Aufried, thanks for your help on the optical part of AFFM, I learned a lot from you. Mark, thank you for the high-resolution SEM measurements and chat. Gerard, thanks for the XPS measurements. Marcel and Kirsten, thanks for ordering the chemicals for me. Richard, thanks for all the support on my computer and network problems. Yvonne, Frans, Erwin, Christian Breukers and Marloes of BPE and Bianca and Tieme of SMCT, thanks for all of your kind help all this time.

Dear Sylvia, the secretary in BPE, thank you so much for your help and support all the time. Your smile and big hug always warm me up; I'll miss them. I would also like to thank the secretaries from SMCT: Izabel, and ex-secretaries, Gerardine, Melissa, Danielle, Marieke for handling all the administrative works.

For the collaborations, I would like to thank Lourdes for being the first one to show me how to prepare monolayer and perform dip-pen nanolithography. I enjoyed all the discussions through Internet while writing the manuscript together and had great time with you and Fernando in Enschede and Dublin. Thank Prof. Jurriaan Huskens and Huaping, for all the useful discussions on the preparing of porous AFM tips. Huaping, thanks for your warm greetings after you went back to China. Dr. Pascal Jonkheijm and Francesca Scaramuzzo, thank you for the collaboration. Francesca, I especially enjoyed the late nights working with you.

Thanks all my officemates in Zuidhorst: Marieke (also my dear paranimf, I really cherish all the moments of chat, watering plants, sharing sweets...etc. with you.), Cicerone (for sharing Romanian information and jokes), Daniele (for the nice pizza and chat) and Malte (for letting me believe there is Santa Claus in the world) and officemates in Hogekamp: Christian Blum (for all the useful discussions and offering me fluorescent proteins), Dianwen (for always willing to share your experience on aligning laser.), Cynthia, Constantin, Raja (especially for your help on the design of thesis cover).

In BPE, I would also like to thank Maryana (for all the nice chat, discussions and help), Yujie (for all the FLIM measurements, help and advises on delivering my concept thesis and the nice chat), Vishnu (for Raman measurements and advises on printing thesis), Tom (for the confocal fluorescence microscope training), Martijn van Raaij (for showing me how to use AFFM), Lily (for all the nice chat and your hospitality), Lanti (for your help on AFM and all the nice chat in both BPE and MnF), Kim (for showing me how to use BioScope AFM), Ine, Mireille, Dodo, Shrirang, Yanina, Alma, Aurel, Hank-Jan, Arun, Roy, Chandra, Tomasz, 
Felicia, Bart, Alex, Liesbeth, Shashank, Remco, Peter, Martijn Stopel, Dirk, Niels and all of you great colleagues in BPE.

In SMCT and MnF, I would also like to thank Shu-Han (for your always energetic laugh and help. I also enjoyed the time with you in Bulgaria), Yiping (also my dear paranimf, thanks for your help on thesis printing, all the nice chat and dinners), Martine (for sharing your experiences and the great time together), Albert (for the solutions of chemistry problems), Denis (for QDs information), Kim (for training me how to use AFM and nice tips on traveling to USA), Arántzazu (for sharing your experiences on experiments), Xing Yi (for all the nice discussions and chat), Xuexin (especially for accompanying to go to NanoNed flagship meetings and conferences), Alessandro (for your kind help on aldehyde monolayer), Alberto (for all the discussions about AFM and also nice travel tips to Madrid), Dorota (for all the porous stamps experiments), Sachine, Huaping, Victoria, Raluca, Laura, Jealemy, Vijay, Srinidhi, Ignacio, Riccardo, Roberto, Peter, Janet, Oya, Tian, Dae Jun, Elisabetta, Francesca Costantini, Francesca Scaramuzzo, Andras, Pieter, Oktay, Nicolai, Musassir, Jordi, Anna, Deniz, Carlo, Melanie, Carmen, Veera, Xuemei, Alessio, Fernando, Lourdes, Soco, Mirko, Henk, Rik and all of you great colleagues in SMCT, MnF and BNT.

Ming Ni, Hsiao-Lei and Li-Hsin, thanks for your support and encouragements during all these years (even more than one third of my life) although we are in different countries. Also thank my friends in Enschede: Amy, Dongpo, Yi-Tzu, Lorraine \& Yung-Ching, Chia-Chi, Chou-Yen, Lelia, Jen Hsuan, Sheng-He, Chin-Huang, Richard Hsieh, Hsiang-Hsu, Hao Gu \& Lanti Yang, Xiao Li \& Zheng Zhang, Tian Gang \& Xin Wan, Qi Chen \& Yujie Ma, Jun Liu \& Nan Li, Yixuan \& Vincent, Yiping, Xiaoying, Jeroen and Eric, I enjoyed all the moments which we chatted, had breakfast/lunch/dinners and played games together. Friends in Delft and Leiden: Bill \& Pei-Hui, Haibo, Chung-Kai, Yan Liu, Vijay and Jan and friends in USA: Briana \& Martin, Cindy \& Kenny, Ching-Hsuan \& Ching-Chih, Noemie, Frank, Chun-Chieh and Wei-Min, thanks for all the nice moments we had been together. In addition, I would like to thank Intervocaal members, especially Marijke, Lon, Ning, Piet and Yulan who always give me encouragements. I had great time while singing together with you on the stage.

Kai-Fan, thank you very much for writing samenvatting and your immense support and love. I am so glad that I can share all my happy and sad moments with you during these years. I would also like to thank your family for their warm encouragements.

In the end, I would like to thank my family and relatives for their unlimited support and love. 親愛的阿公, 阿嬤, 爸爸,媽媽, 亞雅和瑛寶, 謝謝你們這些年來的鼓䅜和支持, 讓我可以在國外追求自 己的夢想, 我愛你們。阿公, 謝謝您從我小時候說故事給我聽, 陪我彈鋼琴, 教我說日文...雖然您在2009 年離開我，但您永遠在我心中，這本論文，特別獻給您!

\section{Chien-Ching Wu}

Enschede, The Netherlands, June 2010 


\section{About the Author}

Chien-Ching Wu was born on 27th July, 1981 in Kaohsiung City, Taiwan. After graduating from Kaohsiung Girls' High School in 1999, she went to study Materials Science and Engineering at National Chiao Tung University in Hsinchu, Taiwan. She obtained her bachelor degree in June 2003. In the same year, she joined the MSc program of Nanoscience provided by Delft University of Technology (TU Delft) and Leiden University in The Netherlands. Her study was funded by ASML-Netherlands Trade and Investment Office (NTIO) Joint Scholarship and TU Delft Dutch Education Learning at Top Level Abroad (DELTA) Scholarship. In August 2005, she obtained her MSc degree from TU Delft under the supervision of Prof. Thomas Schmidt at Leiden University. From May to November 2005, she did her internship in Prof. Mischa Bonn's group at FOM institute-AMOLF. Since December 2005, she started her PhD study under the supervisions of Prof. Vinod Subramaniam and Dr. Cees Otto in Biophysical Engineering Group (BPE) and Prof. David Reinhoudt, Dr. Aldrik Velders and Dr. Mercedes Crego-Calama, in Laboratory of Supramolecular Chemistry and Technology (SMCT). The results of her research are described in this thesis. 
